\title{
POST-DISASTER RECOVERY ASSESSMENT USING REMOTE SENSING IMAGE ANALYSIS AND AGENT- BASED MODELING
}





\title{
POST-DISASTER RECOVERY ASSESSMENT USING REMOTE SENSING IMAGE ANALYSIS AND AGENT- BASED MODELING
}

\author{
DISSERTATION \\ to obtain \\ the degree of doctor at the University of Twente, \\ on the authority of the rector magnificus, \\ prof.dr.ir. A. Veldkamp, \\ on account of the decision of the graduation committee, \\ to be publicly defended \\ on $17^{\text {th }}$ December 2020 at 12.45 hrs
}

by

Saman Ghaffarian

born on $5^{\text {th }}$ June 1985

in Tabriz, Iran 
This thesis has been approved by

Prof.dr. N. Kerle

Prof.dr. T. Filatova

Dr. Debraj Roy

ITC dissertation number 390

ITC, P.O. Box 217, 7500 AE Enschede, The Netherlands

ISBN 90-978-365-5110-6

DOI $10.3990 / 1.9789036551106$

Cover designed by Asal Farahkhah

Printed by Ctrlp

Copyright (C) 2020 by Saman Ghaffarian

UNIVERSITY OF TWENTE.

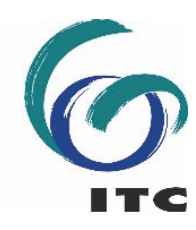




\section{Graduation committee:}

\section{Chairman/Secretary}

Prof.dr. F.D. van der Meer University of Twente

\section{Supervisors}

Prof.dr. N. Kerle

University of Twente / ITC

Prof.dr. T. Filatova

University of Twente / BMS

\section{Co-supervisor}

Dr. D. Roy

University of Twente / BMS

\section{Members}

Prof.dr. V.G. Jetten

University of Twente / ITC

Prof.dr. K. Pfeffer

Prof.dr. M. Herold

University of Twente / ITC

Prof.dr. J. Jokar Arsanjani

Wageningen University

Prof.dr. B. Demir

Aalborg University

TU Berlin 



\section{Acknowledgements}

This work is based on the project initially defined by Prof. Norman Kerle and Prof. Tatiana Filatova and financed by ITC, ESA department. I still remember how enthusiastic and curious I was when I began the journey with Norman's introduction meeting at ITC restaurant. I am grateful for being selected as a Ph.D. candidate at ITC, ESA department for this project, and I express my heartfelt gratitude to Norman and Tatiana, who believe in me and gave me this opportunity.

I want to extend my gratitude to Norman for his support regarding my research focus, scientific inputs, scientific skills and competence within academia, and dedication in helping me during these years. Also, to thank Tatiana for her patience in teaching me the economic-related topics and her support and scientific inputs. I would like to also thank Dr. Debraj Roy, who joined the supervisory team in the last year of my Ph.D., for his daily supervision; I couldn't complete the last part of my Ph.D. without your supports. I want to thank collaborators from other ITC departments, universities and organizations in my Ph.D. studies: Dr. Edoardo Pasolli, Prof. Jamal Jokar Arsanjani, Dr. Malte Lech, Dr. Gerald Leppert, Dr. Raphael Nawrotzki, Dr. Monica Kuffer, and Ali Rezaie Farhadabad.

I would like to thank my friends and colleagues at ITC and in particular in the ESA department for their support and advice over these years: Sobhan, Fardad, Siavash, Elham, Islam, Hakan, Mohammadreza, Sanaz, Azin, Yakob, Vasily, Oscar, Ploy, Sofia, Evelien, Bastian, Nicoletta, Yaser, Jonathan, Biao, Yan. I also acknowledge the support of the European Space Agency and Digital Global Foundation by accepting my proposal and granting free satellite imagery. I am grateful for the colleagues and people in the Philippines (Manila and Tacloban cities) that helped me in my fieldwork.

A big thank you to my parents; I owe everything to you. To my wife, Asal, thank you for your love, inspiration, endless support, and encouragement. 


\section{Table of Contents}

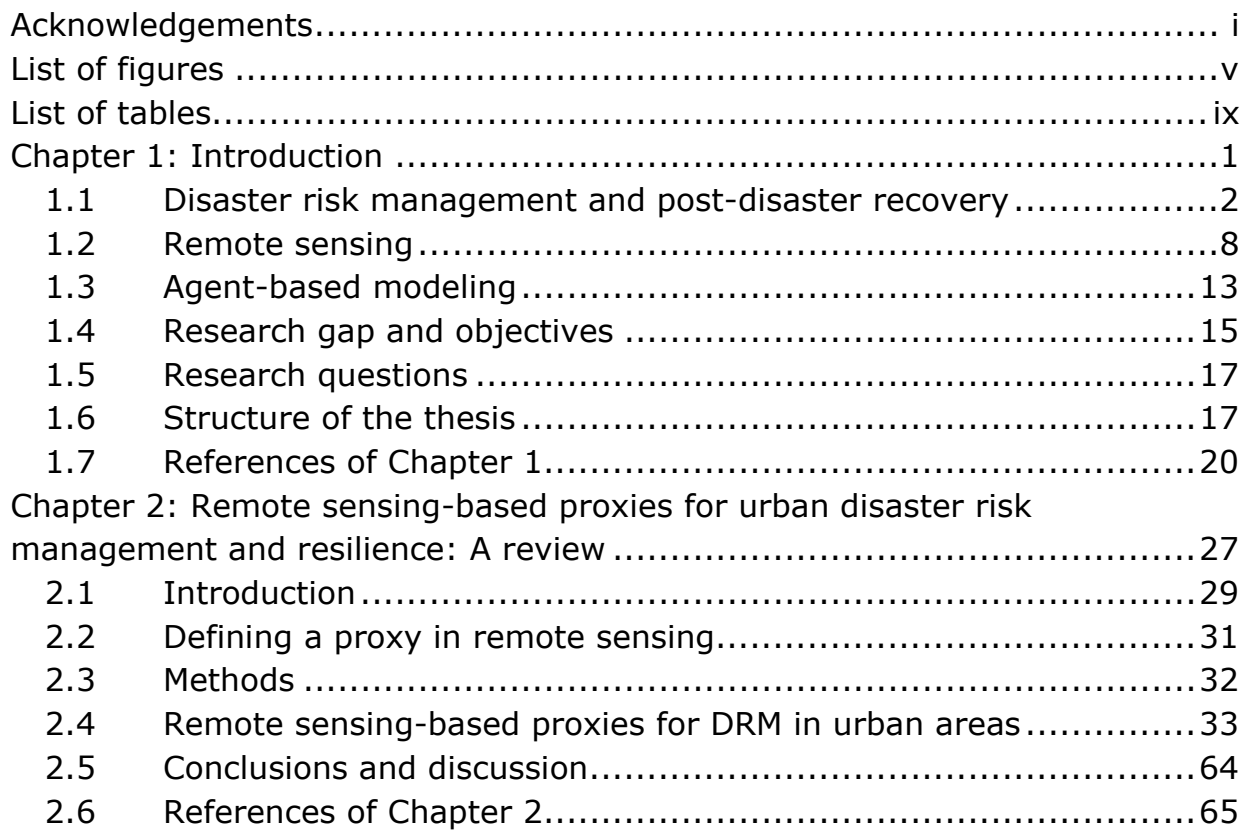

Chapter 3: Conceptual framework for post-disaster recovery assessment

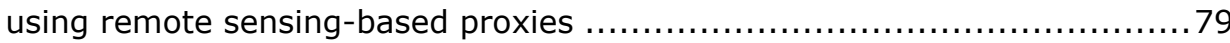

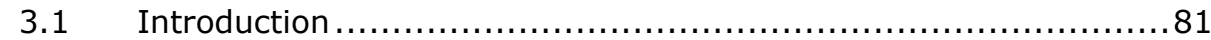

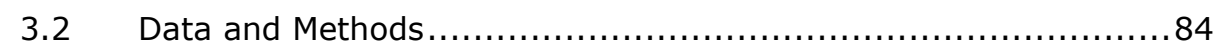

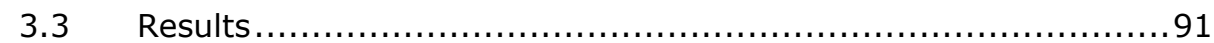

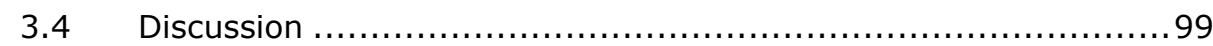

3.5 Conclusions................................................... 100

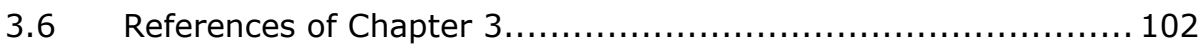

Chapter 4: Towards post-disaster debris identification for precise damage and

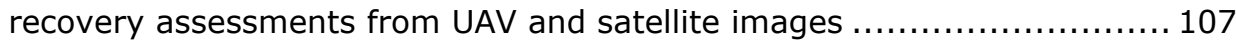

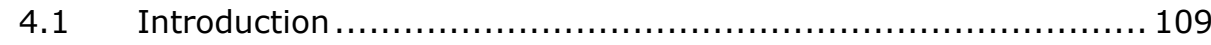

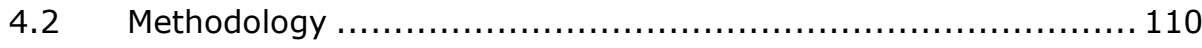

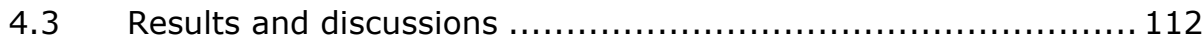

4.4 Conclusions and future work ..................................... 115

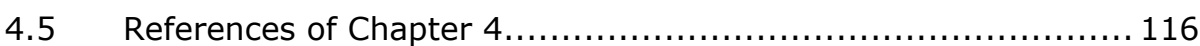

Chapter 5: Post-disaster building database updating using automated deep learning: An integration of pre-disaster OpenStreetMap and multi-temporal

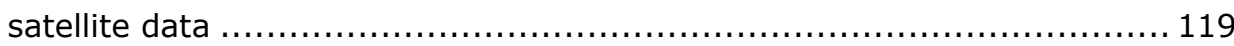

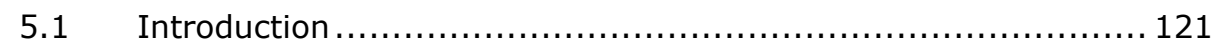

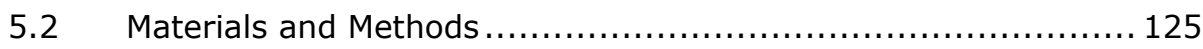

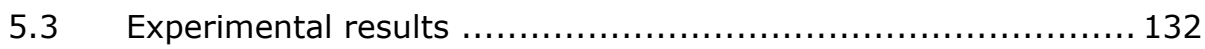

5.4 Experimental results and discussion............................. 135

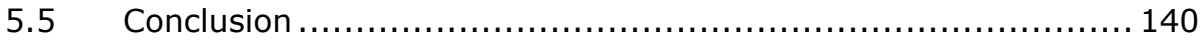




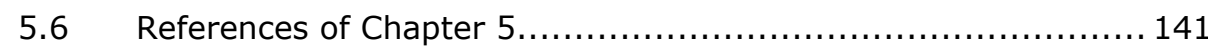

Chapter 6: Post-disaster recovery monitoring using Google Earth Engine.. 149

$6.1 \quad$ Introduction ......................................................... 151

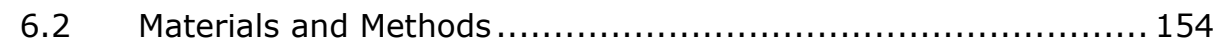

$6.3 \quad$ Results and Discussion ............................................ 160

6.4 Discussion and Conclusions ........................................ 164

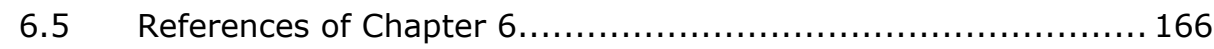

Chapter 7: Post-disaster recovery agent-based modeling using remote

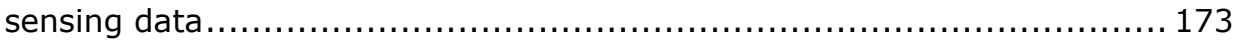

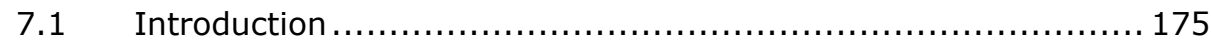

7.2 Methods .............................................................. 177

7.3 Results and discussion ............................................. 182

7.4 Conclusions........................................................... 188

7.5 Appendix A. the ODD+D description of the PDR ganet-based model .................................................................. 189

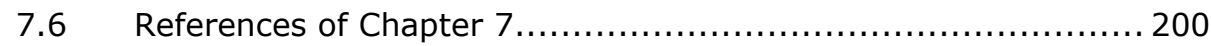

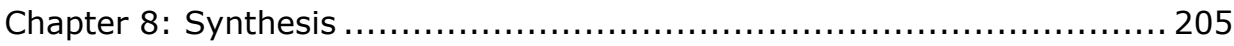

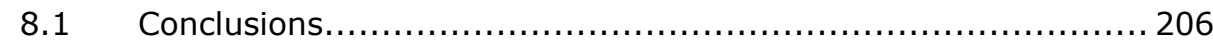

8.2 Reflections and outlook ........................................... 210

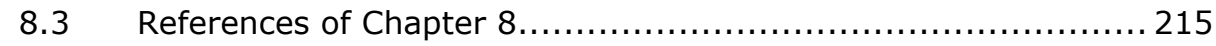

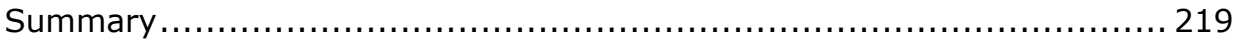

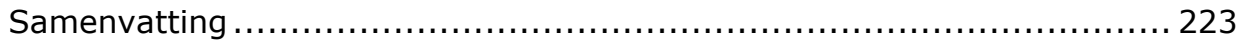

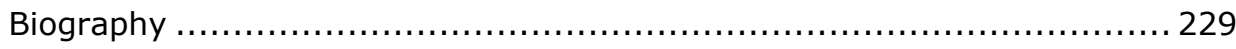




\section{List of figures}

Figure 1.1 Human impacts and economic losses of disasters between 19982017 (adapted from CRED $(2018)$ ) .........................................

Figure 1.2 Disaster risk management cycle (adapted from Coppola, 2015) ..3 Figure 1.3 Recovery vs Rehabilitation vs Reconstruction (modified from UNDP, 1993)

Figure 1.4 Importance of resilience in post-disaster recovery process/curve.

Figure 1.5 Recovery indicator outputs using satellite images. Retrieved from Brown et al. (2010a). .... 10

Figure 1.6 Accessibility assessment of roads and bridges. Retrieved from Brown et al. (2011).

Figure 1.7 Thermal scan images to measure the quality of housing. Retrieved from Costa Viera and Kerle (2014).

Figure 1.8 Agent-based modeling visualization. Retrieved from Galan et al. (2009). .14

Figure 2.1 Number of annual publications on remote sensing-based proxies for Disaster Risk Management (DRM). Papers with a focus on two DRM areas, e.g., both damage and recovery assessments, are counted on both of them separately. ... 34

Figure 2.2 Number of developed remote sensing-based proxies for DRM in each environment (Built-up, Economic, Social, Natural). The proxies that are used interchangeably in more than one area, e.g., damage and recovery assessments, are counted on all of the used categories separately. However, the green colored bar shows the total number of unique RS-based proxies for each environment. .34

Figure 2.3 Examples of remote sensing-based built-up proxies. (a) Roof with dislocated tiles, (b) cracks in concrete façade, (c) cracks and hole in brick façade (Galarreta et al. 2015), (d) inclined building (Galarreta et al. 2015; Tamkuan and Nagai 2017), (e) debris, rubble piles, spalling (Vetrivel et al. 2016a) and (f) façade windows symmetry (Tu et al. 2017b).

Figure 3.1 The conceptual framework connecting post-disaster recovery assessment using remote sensing-based proxies with resilience assessment and impact evaluation. .85

Figure 3.2 Municipalities included in this study, and overview of Barangay 69. Notes: Brgy. = barangay; SIMPLE = Sustainable Integrated Management and Planning for Local Government Ecosystems intervention; GIZ = German agency for technical cooperation. 86

Figure 3.3 Framework for the extraction of proxies using high resolution satellite images for each region of interest (ROI) at each time step (e.g., predisaster, T0). 90

Figure 3.4 $A 0, A 1, A 2, A 3$ : Original very high resolution WorldView2, GeoEye1, GeoEye1, and WorldView2 satellite images, respectively, acquired over Barangay 69, Tacloban city from 8 months before (T0), right after (T1), 2 years 
(T3) and 4 years after Typhoon Haiyan (T3), respectively; B0,B1,B2,B3: LC classification result for the four time epochs; $\mathrm{C} 0, \mathrm{C} 1, \mathrm{C} 2, \mathrm{C} 3$ : corrresponding pie charts show ditribution of the LC classes. The area denoted by the blue circles in $A 1$ and $B 1$ shows the shadowed area in the image.

Figure 3.5 A0,A1,A2,A3: Original very high resolution WorldView2, GeoEye1, GeoEye1, and WorldView2 satellite images, respectively, acquired over Barangay 69, Tacloban city, 8 months before (T0), right after (T1), 2 years (T3) and 4 years after Typhoon Haiyan (T3), respectively; B0,B1,B2,B3: LU classification result for the four time epochs; $\mathrm{C} 0, \mathrm{C} 1, \mathrm{C} 2, \mathrm{C} 3$ : corrresponding pie charts show ditribution of the LU classes. The area denoted by the blue circles in $A 1$ and $B 1$ shows the shadowed area in the image.

Figure 4.1 a) Track of Typhoon Haiyan over the Philippines, b-c) Overview of Tacloban city. 1-5) The selected UAV images for one week after the disaster from the study area. 110

Figure 4.2 1-5) UAV images and their corresponding HOG vector results for the denoted regions. 113

Figure $4.3 \mathrm{a}-\mathrm{j}$ ) Very high-resolution satellite images respectively for before disaster, 2, 3, 5, 7 days, 4 and 5 weeks, and 2, 8, and 9 months after the disaster. 115

Figure 5.1 The framework proposed in this paper for post-disaster building database updating. Notes: $\mathrm{V}-\mathrm{HOG}=$ Variation of Histogram of Oriented Gradients; EDI = Edge Density Index; CRF = Conditional Random Field. .. 125 Figure 5.2 Example of the co-registration of the OSM building map and satellite images for Tacloban city, the Philippines. (a) Pre-disaster satellite image, (b) original OSM building map, and (c) modified OSM building map. The areas denoted by red boundaries show the effect of the refinements on OSM map data.

Figure 5.3 (a-c) Pre-disaster data in terms of (a) multispectral image, (b) VHOG and (c) edge detection image; OSM building map denoted with red lines. (d-f) Event time data in terms of (d) multispectral image, (e) V-HOG and (f) edge detection image; . result of Step 3 denoted with red lines

Figure 5.4 The proposed ResUnet-CRF framework.

Figure 5.5 An overview of the Philippines showing the path of Typhoon Haiyan (a) and the location of Tacloban city (b). Pre-disaster image (c), and image acquired three days after the disaster (d).

Figure 5.6 The results of the proposed method, test images and pre-disaster images with OSM building boundaries (yellow). Column A: Pre-disaster (8 months before Haiyan) images with OSM building boundaries in yellow. Column B, Images \#1-5 taken 3 days after Haiyan and Images\#6-10 taken 4 years after Haiyan. Column C: the reference image for buildings, in which white and black colors represent the building and background pixels, respectively. Column D: detected buildings for test images. Green, red and blue represent TP, FP and FN, respectively. 
Figure 5.7 (a) Original test Image\#1, (b) ResUnet-CRF, (c) ResUnet, and (d) ResUnet without fine-tuning results. The areas within the yellow boundaries denote the dark green buildings to stress the effect of the CRF in the final result. The areas within the purple boundaries denote the inaccuracies in the results extracted using the network initially trained without fine-tuning. Green, red and blue pixels represent TP, FP and FN, respectively. 140

Figure 6.1 An overview of the Philippines showing the path of Typhoon Haiyan and the location of Leyte island. Pre-event image and an image acquired three days after the disaster for Tacloban. 155

Figure 6.2 The proposed framework for post-disaster recovery monitoring. 157

Figure 6.3 (a-e) Landsat image composites acquired over Leyte island for T0T4 times, respectively, $(f-j)$ land cover classification maps produced with GEE for T0-T4 times, respectively. Red rectangles show the status of the relocation site (northern part of Tacloban) for T0-T4 times. Non-tv-Non-tree vegetation.

162

Figure 6.4 Per class land cover percentage for Leyte island for T0-T4. Nontv-Non-tree vegetation. 163

Figure 6.5 (a) Municipality level damage map for T1 and (b-d) the recovery maps for T2-T4 times after Typhoon Haiyan for Leyte island, respectively.164 Figure 7.1 The overview of Tacloban, the Philippines, and the satellite image for the modelled urban area acquired before Haiyan..... 178

Figure 7.2 Mean standard deviation utility satisfaction of the families residing in formal (FH) and informal (IH) urban areas for different time steps in the model, in which each step is equal to a month and the time step = 1 is the Haiyan disaster moment. 183

Figure 7.3 (a) Pre-Haiyan high-resolution satellite image of central Tacloban, (b-f) spatial distribution of the mean utility satisfaction for IHs and FHs for steps $0,2,5,10$, and 19, respectively. The areas denoted with a circle and a rectangle denote informal and formal settlements, respectively. Each time step is equal to a month, and step = 1 is the Haiyan disaster moment..... 184

Figure 7.4 Percent of HHs work in high income versus low-income jobs in the post-disaster recovery process. Each time step is equal to a month, and step $=1$ is the disaster moment. 184

Figure 7.5 Mean and standard deviation utility satisfaction of the families residing in formal (FH) and informal (IH) urban areas for different time steps with the presence of relocation site. Each time step is equal to a month and the time step $=1$ is the disaster/Haiyan moment..... 185

Figure 7.6 (a) Pre-Haiyan high-resolution satellite image of Tacloban urban area, (b-h) spatial distribution of the mean utility satisfaction for the His and FHs with the occupation ratio of the relocation site for steps $0,2,3,4,5,10$ and 19 , respectively, and (i) is the relocation site occupied and unoccupied ratio for pre-and post-disaster situations. Each time step is equal to a month, and step $=1$ is the disaster moment. 
Figure 7.7 The mean and standard deviation utility satisfaction produced by the PDR model with different employment ratios $(0.5,0.7,0.8,0.92)$ for the IH (a) and FH (b) in different time steps. Each time step is equal to a month, and step $=1$ is the disaster moment......................................... 188

Figure 7.8 Conceptual flow framework of the PDR ABM...................... 191

Figure 7.9 The probability distribution of the job sectors for IH (a) and FH (b) over time. Each time step is equal to a month, and step $=1$ is the disaster moment........................................................................ 193

Figure 7.10 The probability distribution of the distance to the workplace (i.e. service job type) for FHs. 195 


\section{List of tables}

Table 1.1 The four phases of DRM (adapted from UNISDR, 2009) .............3

Table 2.1 Remote sensing-based built-up proxies for urban DRM, Buildings category. Mono and Multi refer to mono-temporal and multi-temporal RS data that are used for the extraction, respectively ................................ 38

Table 2.2 Remote sensing-based built-up proxies for urban DRM, Transport category. Mono and Multi refer to mono-temporal and multi-temporal RS data, respectively, used for the extraction, respectively.

Table 2.3 Remote sensing-based built-up proxies for urban DRM, Others category. Mono and Multi refer to mono-temporal and multi-temporal RS data that used for extraction, respectively.

Table 2.4 Remote sensing-based economic proxies for urban DRM, Macro, regional and urban economics category. Mono and Multi refer to monotemporal and multi-temporal RS data that used for extraction, respectively.

Table 2.5 Remote sensing-based economic proxies for urban DRM, Logistics category. Mono and Multi refer to mono-temporal and multi-temporal RS data that used for extraction, respectively. 52

Table 2.6 Remote sensing-based social proxies for urban DRM, Services and infrastructures category. Mono and Multi refer to mono-temporal and multitemporal RS data that used for extraction, respectively. 55

Table 2.7 Remote sensing-based social proxies for urban DRM, Socioeconomic status category. Mono and Multi refer to mono-temporal and multitemporal RS data that used for extraction, respectively. 58

Table 2.8 Remote sensing-based proxies for the natural environment of urban DRM. Mono and Multi refer to mono-temporal and multi-temporal RS data that used for extraction, respectively.

Table 3.1 Satellite images used in this study.................................. 87

Table 3.2 The proxies used for the recovery assessment.....................89

Table 3.3 The LC classification accuracies for T0, T1, T2 and T3 time epochs for Barangay 69. User and producer accuracies and corresponding errors are computed across the study area from the confusion matrices. PA - Producer's Accuracy; UA - User's Accuracy; OA - Overall Accuracy; N.S - Number of Smaples used for accruacy assessment. .95

Table 3.4 The LU classification accuracies for T0, T1, T2 and T3 for Barangay 69. User and producer accuracies and corresponding errors were computed across the study area from the confusion matrices. BareL: Bare land; FB: Formal built-up area; ImS: Impervious surface; LSI: Large scale industry; IS: Informal settlement; PA - Producer's Accuracy; UA - User's Accuracy; OA Overall Accuracy; N.S. - Number of Smaples used for accruacy assessment. .96

Table 3.5 The extracted results for the selected proxies for Barangay 69, Tacloban. WV2: WorldView2; GE1: GeoEye1. .97 
Table 5.1 The targeted post-disaster building detection scenarios for each selected test images. ................................................... 134

Table 5.2 The parameters and threshold values used to do the experiments.

135

Table 5.3 Numerical results of the proposed post-disaster building database

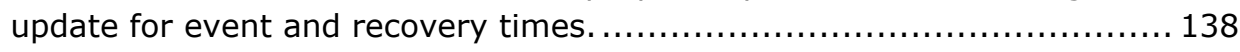

Table 6.1 Satellite images used in this study............................ 155

Table 6.2 Description of the selected land cover classes.................. 156

Table 6.3 The land cover classification accuracies for T0, T1, T2, T3, and T4 time epochs for Leyte island. PA-producer's accuracy; UA-user's accuracy; OA-overall accuracy; Non-tv-Non-tree vegetation. ..................... 161

Table 7.1 Satellite images used in this study............................ 179

Table 7.2 Explanation of the variables used for computing the utility satisfaction of the agents in each step.................................. 194

Table 7.3 Explanation of the variables, their values and data sources used for the base PDR scenario. 199 
Chapter 1 - Introduction 


\subsection{Disaster risk management and post-disaster recovery}

A disaster is a serious event that disrupts the functioning of a community or a society in a way that they cannot cope with using their own resources. It causes widespread human, physical, economic, and environmental losses and impacts (UNISDR 2009). The combination of hazards, vulnerability, and the inability to reduce the potential negative consequences of risk results is a disaster (IFRC 2016).

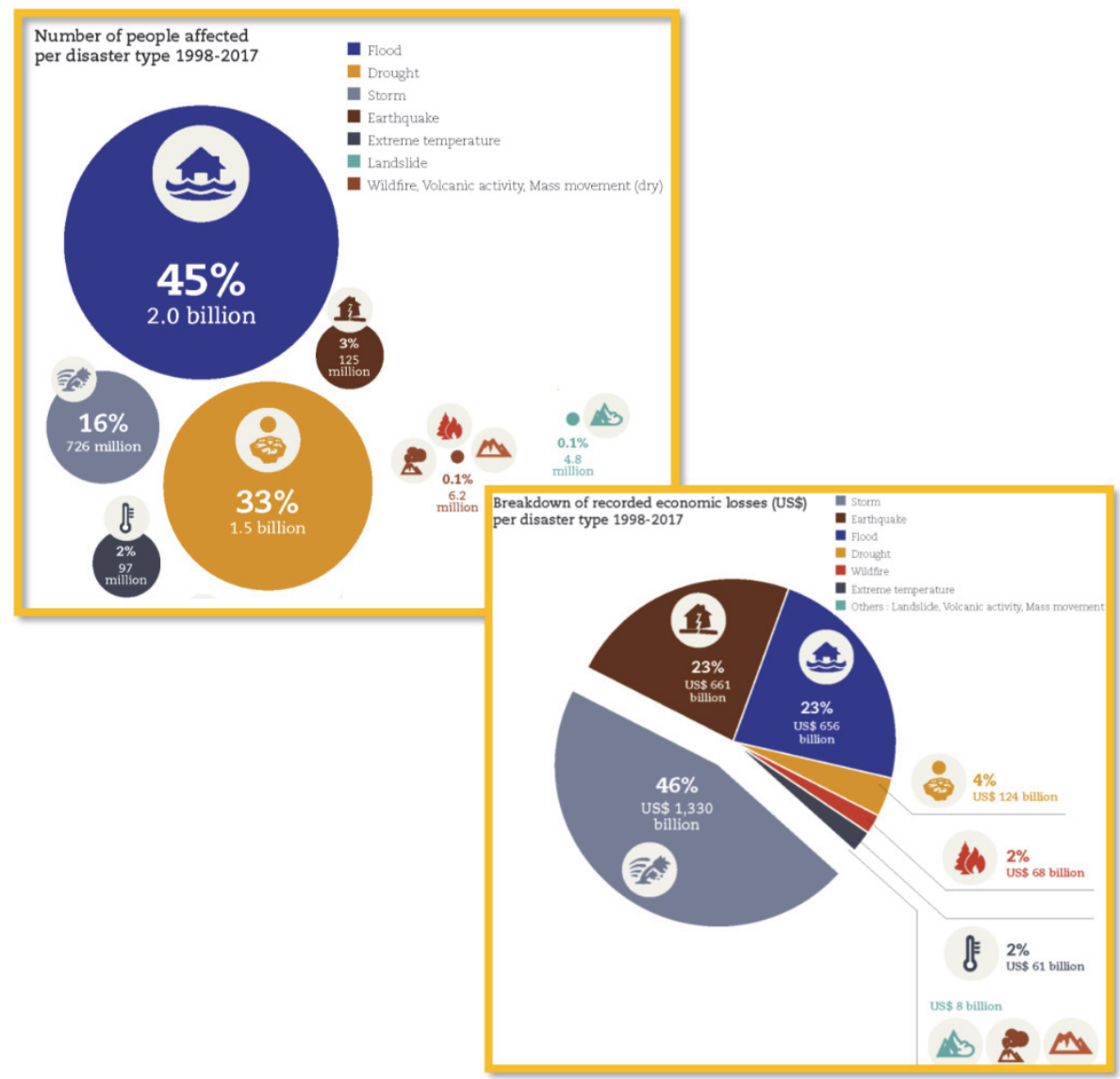

Figure 1.1 Human impacts and economic losses of disasters between 1998-2017 (adapted from CRED (2018))

Natural disasters can cause massive problems for communities, societies, and economies, and devastating impact on infrastructures, firms, and people in the affected region (Cole et al. 2013). Between 1998 and 2017, more than 5.7 billion people were affected, and more than 1.3 million people were killed by disasters. A total loss of US\$2.9 trillion was reported over the period of 1998 
to 2017 (CRED 2018) (see Figure 1.1). These statistics clearly demonstrate the significance of the management of disasters, from rapid response to the complete recovery process after any disaster event.

Disaster Risk Management (DRM) aims to avoid, lessen, and transfer the adverse effects of hazards through activities and measures for prevention, mitigation, and preparedness (UNISDR 2009). Four main phases in a disaster cycle are considered in DRM studies; response, recovery, mitigation, and preparedness (Coppola 2015) (Figure 1.2).

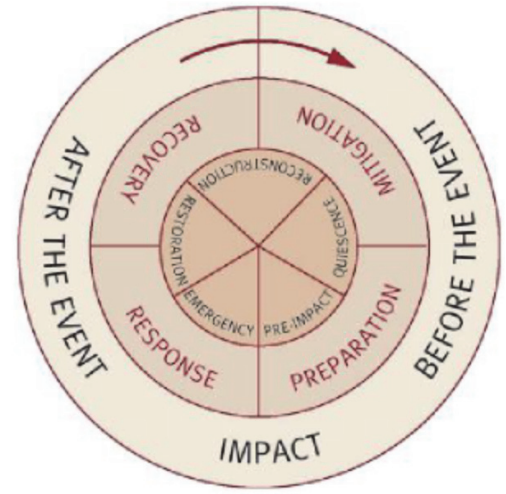

Figure 1.2 Disaster risk management cycle (adapted from Coppola, 2015)

Table 1.1 The four phases of DRM (adapted from UNISDR, 2009)

\section{The four phases of DRM}

Response: The provision of emergency services and public assistance during or immediately after a disaster to save lives, reduce health impacts, ensure public safety, and meet the basic subsistence needs of the people affected.

Mitigation: The lessening or limitation of the adverse impacts of hazards and related disasters.
Recovery: The restoration, and improvement where appropriate, of facilities, livelihoods and living conditions of disaster-affected communities, including efforts to reduce disaster risk factors.

Preparedness: The knowledge and capacities developed by governments, professional response and recovery organizations, communities, and individuals to effectively anticipate, respond to, and recover from, the impacts of likely, imminent or current hazard events or conditions.

Table 1.1 provides generic definitions for the four phases of DRM. Of the different DRM phases, mitigation and preparedness take place before a disaster, while the response and recovery phases take place during and after the disaster event. After a disaster, actions are taken to save lives and prevent 
further property damage (response phase) and then to return to a normal or even better condition (recovery phase). In the mitigation phase, actions are taken to prevent a disaster, reduce the chance of a disaster happening, or reduce the damaging effects of unavoidable disasters, while in the preparedness phase, plans are considered or preparations made to save lives and to help the response of a disaster.

Post-disaster recovery is the process of reconstructing communities in all their aspects (physical, economic, social, and environmental) to return life, livelihoods, and the built environment to their pre-impact or even better states (Burton et al. 2011). Conventionally, recovery was considered as a predictable and orderly process (Haas et al. 1977). However, recent studies demonstrated that the recovery process is more complex (Brown et al. 2015). The complexity of the recovery process is basically because of being a multi-dimensional process; indeed, it needs numerous sectors, stakeholders, policymakers, and so on to take a role and responsibility. Reconstruction and, consequently, recovery starts after the disaster has happened; therefore, governments and disaster planners have to make decisions and act quickly. However, on the one hand, reconstruction of buildings is only the physical part of the recovery, i.e. it omits other important sides, such as economic, social and environmental aspects. There are several socio-economic factors that influence the recovery process and its rate from early stage such as social interactions (e.g. social cohesion) (Townshend et al. 2014), or business recovery (Rose and Krausmann 2013). For example, manufacturers or service providers do not return to the reconstructed region without existing consumers for their products, and workers do not return without having appropriate jobs.

On the other hand, there are many other vital factors of recovery, such as functional analysis of the reconstructed physical factors. For instance, does a newly reconstructed building represent a successful recovery process even if it is empty and nobody lives in it? Or an equipped hospital with skilled medical staff and a sufficient number of beds to support injured people, but without electricity? These two examples, which are actual examples of what happens after a disaster, confirm the importance of considering functional recovery in the post-disaster recovery process. In addition, functional recovery analysis can show the changes in functions (i.e., residential, commercial, education and etc.). Therefore, post-disaster recovery is a compound process and is vital for communities hit by disaster to survive and return to normal living conditions.

The term recovery is often confused with reconstruction and rehabilitation. However, there are differences between these terminologies. Rehabilitation, reconstruction, and recovery all start after a disaster. Rehabilitation is the process of enabling necessary services to resume functioning, help victims for preliminary repayments of physical damages and community infrastructures, 
restore basic economic activities, and support the social and psychological wellbeing of the survivors (UNDP, 1993). Primarily rehabilitation actions are taken for enabling the affected community and populations to more or less resume their essential normal life, or in other words, to stand up and survive. Reconstruction refers to the restoration of all services, infrastructures, and rebuilding of damaged physical structures, such as individual buildings, schools, hospitals, which help with the revitalization of the economy and the restoration of social and cultural life. The recovery phase is defined as the actions for the period after the emergency phase and includes both rehabilitation and reconstruction and full functional recovery (Figure 1.3)(UNDP 1993). However, it varies over time and space due to several factors, such as socio-economic and political ones, and because of the multitude of decisions that are made before, during and after a disaster (Olshansky et al. 2006).

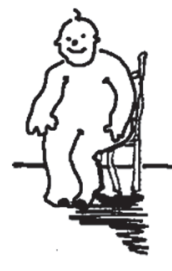

1- Normal

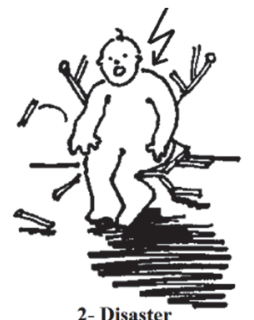

2- Disaster

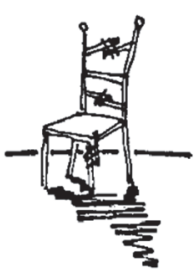

3- Rehabilitation

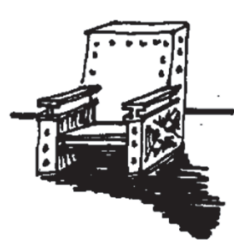

4- Reconstruction

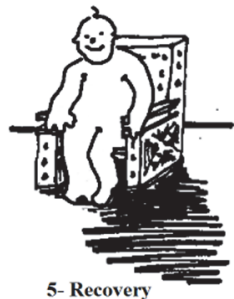

5- Recovery

Figure 1.3 Recovery vs Rehabilitation vs Reconstruction (modified from - UNDP, 1993)

Another factor that is essential in post-disaster recovery and influences its duration and quality is resilience (Platt et al. 2016; Unisdr 2015). Resilience is "the ability to absorb change and disturbance and still maintain the same relationships that control a system's behavior," and was first defined by Holling (1973) in the ecology domain. Then, Timmerman (1981) used the term resilience in a disaster context and described it as the measure of the capacity of a system, or part of a system, to absorb or recover from a damaging event. Since resilience entered in the disaster field researchers have tried to complete and revise its definition. They also tried to include it in the disaster risk assessment equation, not only in natural disaster studies but also in other studies, such as in the social, economic, and environmental fields. In the natural disaster domain, resilience has been incorporated into the risk equation (Eq.1), and is mostly accepted as "the ability of a system, community or society exposed to hazards to resist, absorb, accommodate to and recover from the effects of a hazard in a timely and efficient manner, including through the preservation and restoration of its essential basic structures and functions" which was defined by the United Nations Office for Disaster Risk Reduction (UNISDR, 2009). 
Risk $=\frac{\text { Hazard } * \text { Exposure } * \text { Vulnerability }}{\text { Resilience }}$

Resilience includes inherent conditions, allowing communities to absorb impacts and cope with an event. Resilience also encompasses post-event processes that would enable communities to reorganize, change, and learn in response to an event (Cutter et al. 2008). Thus, to enhance a community's resilience to natural hazards is to improve its capacity to anticipate threats, reduce its overall vulnerability, and to allow the community to recover from adverse impacts when they occur. Decades of hazards and disaster research have offered extensive findings within this context (Burton 2014; Kates et al. 2006; Sadiq et al. 2019; Tiernan et al. 2019). The threat of natural disasters will continue, but their consequences can be reduced if communities and people increase their resilience (Council 2012; Jones and Ballon 2020). However, measuring the resiliency of a community has not yet been sufficiently addressed, and it is a challenging topic for DRM researchers.

Recently, the Sendai Framework has been defined for disaster risk reduction as a roadmap to make communities safer and resilient to disasters (UNISDR 2015). It proposes to use the post-disaster as a window of opportunity to build back better, increasing the resilience of the community while reducing its preexciting vulnerability. Accordingly, the aim of the post-disaster recovery will be not only to return the community to normal/pre-disaster situation, but also to improve the pre-existing physical, social, economic, and environmental conditions.

Resilience is increasingly becoming a ubiquitous concept in many disciplines, such as economic, sociology, psychology, and healthcare. It has also been considered as an emerging research topic in disaster risk management in recent decades. Figure 1.4 shows the relationship between resilience and postdisaster recovery by comparing recovery curves of a more resilient community with ordinary communities, demonstrating that the resilient communities recover faster and even better than other communities. Since there is a strong link between resilience and post-disaster recovery, resilience is frequently used as a guiding concept for developing policies, plans, and programs to deal with a diverse array of natural and human-made disasters that are progressively increasing in frequency and severity (Sharifi and Yamagata 2016). 


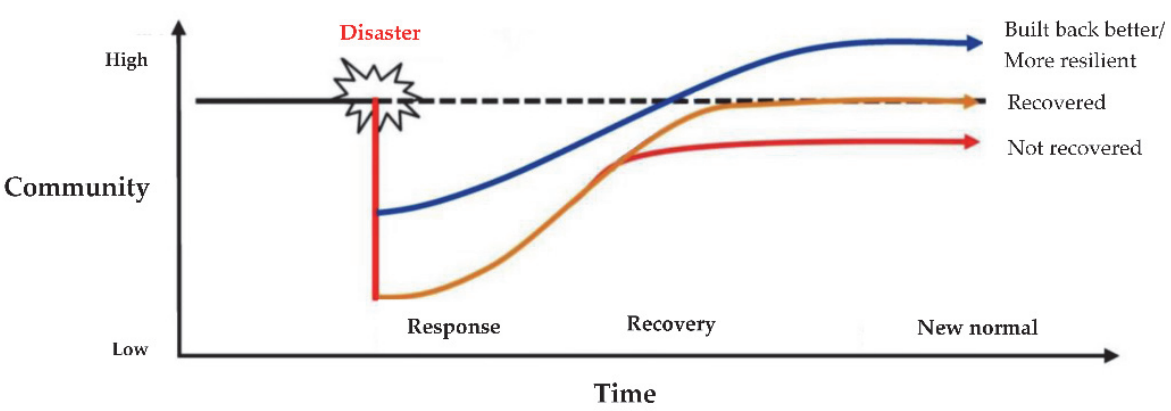

Figure 1.4 Importance of resilience in post-disaster recovery process/curve.

Consequently, resilience-centered management has become a policy objective in the United States and worldwide (Bakkensen et al. 2016). For instance, the U.S. department of Housing and Urban Development launched a \$1 billion initiative to increase natural disaster resilience across communities, and the Philippines government planned to launch $\$ 8.2$ billion for the recovery from the 2013 Typhoon Yolanda and to increase resilience (Lum and Margesson 2014). Hence, resilience to natural disasters is an important policy objective for many governments.

Since the term has been defined in the DRM field, researchers have tried to measure the resilience of communities (Manyena 2006; Revet 2012). Resilience cannot be measured directly, similar to the recovery process, and thus several researchers have attempted to identify and group the related indicators (overall disaster or socio-economic resilience) (Rose and Krausmann 2013). For instance, Cutter et al. (2010) used housing capital, equitable incomes, employment, business size, and position access as indicators for the economic resilience of a community. Bruneau et al. (2003) created a conceptual framework for the quantitative assessment and enhancement of the seismic resilience of the community considering engineering-based resilience. They focused on critical infrastructures for the resilience measurement of both physical and social systems. Their measurement is based on reducing failure probabilities, consequences from failures, and time to recovery. They concluded that their proposed framework makes it possible to assess and evaluate the contribution to seismic resilience of various activities. Mayunga (2007) analyzed the resilience with a capital-based strategy. The major challenge of their proposed method is how to measure each form of capital adequately, due to having a relatively broad framework. They concluded that it is practically not possible to measure all the dimensions of each type of capital, partly because of the limitation of data availability. In a different study, Maclean et al. (2014) studied disaster resilience, focusing on social components, and they generated six key indicators, including engagement of people and places, the presence of community infrastructure, community 
networks, and governance. Furthermore, Townshend et al. (2014) mentioned the importance of social cohesion in community resilience and pointed out a potential link between place-based social cohesion and resilience. Several studies also exist for disaster resilience measurements based on content analysis (Jordan et al. 2011), vulnerability analysis (Burton et al. 2002), production theory macroeconomics (Rose 2009), and key infrastructure resilience (Fisher et al. 2010). Some other researchers attempted to create a resilience index, and key indicators to generate an overall and complete indicator list for disaster socio-economic resilience (Norris et al. 2008; Rose and Krausmann 2013; Sharifi and Yamagata 2016).

Some studies defined formulas to quantitatively measure socio-economic resilience. For instance, socioeconomic resilience quantitatively is defined by Hallegatte et al. (2016) to measure the ability of an economy to minimize the impact of asset losses on wellbeing, and one part of the ability to resist, absorb, accommodate and recover in a timely and efficient manner from asset losses. In order to show the effect of socio-economic resilience in computing disaster risk to welfare, they used the following equation:

Risk to welfare $=\frac{\text { Expected asset losses }}{\text { Socioeconomic resilience }}=\frac{\text { Hazard } * \text { Exposure } * \text { Asset vulnerability }}{\text { Socioeconomic resilience }}$

Also, they measured socioeconomic resilience to floods and generated scorecards using their developed model for 90 countries.

\subsection{Remote sensing}

Remote sensing can provide a valuable source of information at each phase of the DRM cycle, helping to understand the spatial domain from a wide range of areas to small scales, supporting scientists and authorities with objective information for decision making. One of the important challenges with disaster management is the unpredictability of hazard events and their magnitude, which does not allow for a single all-encompassing solution to be developed and explored (Joyce et al. 2009). Remote sensing provides various types of data in terms of spatial, spectral, and temporal resolutions and scales. Therefore, remote sensing platforms potentially can provide data required, and answer information needs for each phase of DRM. For instance, in the mitigation phase of DRM, and to assist risk reduction, remote sensing has been used to identify the hazard-prone regions associated with flood plains, coastal inundation and erosion (Klemas 2014), landslides (Pradhan et al. 2006), and active faults (Dalati 2005).

Furthermore, it has been employed to verify hazard models by measuring the location and magnitude of actual events (Joyce et al. 2009). Meteorologists use remote sensing imagery to forecast weather (Liu et al. 2019; Nashwan et 
al. 2019; Thies and Bendix 2011), and produce warnings of potentially severe weather events. Indeed, remote sensing can provide critical information for the public and emergency responders that can assist decision making around short term preparedness. In the response phase of DRM, remote sensing provides a rapid method of assessing damages, most affected areas, and significant information such as where key transport and other infrastructure links have been damaged or destructed (Kerle 2011; Kerle and Hoffman 2013). Besides, by developing new technologies in sensors and computer-based systems, remote sensing data are becoming more readily available. Due to the recognition of the significance of the information that remotely sensed imagery could provide, some satellites are even addressing at least partially the DRM and emergency response needs (Joyce et al. 2009). Furthermore, some systems have been developed, such as the International Charter "Space and Major Disasters", to provide space data acquisition and delivery to those affected by natural or man-made disasters, and even some organizations provide information from ground-based sensors in addition to satellite and airborne sensors' data, such as Copernicus.

Recently, remote sensing data have been employed to study all phases of the DRM cycle with the growing availability of their various types. This is due to increasing the spatial, spectral, and temporal resolution of the remote sensing imagery. Several automatic damage assessment methods based on change detection techniques have been developed (Kerle et al. 2019). For example, post-disaster damage assessment such as for buildings, roads, infrastructure has been carried out using remote sensing data sets such as satellite (Duarte et al. 2018b; Vetrivel et al. 2016b), aerial (Duarte et al. 2018a; Galarreta et al. 2015; Nex et al. 2019; Vetrivel et al. 2016a), SAR images (Bell et al. 2019; Chen and Sato 2013; Dadhich et al. 2019; Yulianto et al. 2015) and LiDAR data (Rastiveis et al. 2015). In contrast, only few studies exist on the use of remote sensing data sets to monitor and evaluate the recovery phase of the DRM cycle (Brown et al. 2008; Platt et al. 2016). Furthermore, most of the developed methods for recovery assessment are manual, and only a few are semiautomatic, which are based on conventional computer vision models (Brown et al. 2010b). However, the need for developing rapid, automatic, and robust methods for post-disaster recovery assessment is demonstrated in the literature (Joyce et al. 2009). One of the reasons for the limited number of studies on post-disaster recovery assessment compared with damage assessment is that the recovery process cannot be assessed in a direct manner in most of the cases (note: if the damage is considered in its all aspects, not only physically, it could also not be assessed directly). Accordingly, the need for indicators and proxies is an obstacle of entering remote sensing and computer vision societies to this field. 
Remote sensing data have been rarely used for post-disaster recovery monitoring and evaluation. For instance, Curtis et al. (2010) employed video data sets for monitoring and assessing the recovery processes of Hurricane Katrina. They utilized videos to gather information about house conditions and occupancies. They finally concluded that their method is an efficient tool for collecting neighborhood data after a disaster. However, several critical places may not be accessible to collect video data in a post-disaster situation.

Brown et al. (2010a) used indicator-based methods to monitor and evaluate the post-disaster recovery assessment based on high-resolution remote sensing imagery, particularly IKONOS and QuickBird satellite images, in addition to field surveys and internet-based statistic data sets. They utilized image processing techniques for change detection in the region, such as land cover changes, building-based recovery/reconstruction analysis. Then, change detection methods were used to support and extract changes at indicator levels. They also used field surveys as complementary information for remote sensing image analysis (Figure 1.5). However, their developed indicators in this study did not robustly represent the entire recovery process, for example lacking functional recovery indicators. Furthermore, their proposed image processing techniques are not efficient, e.g. maximum likelihood classification.

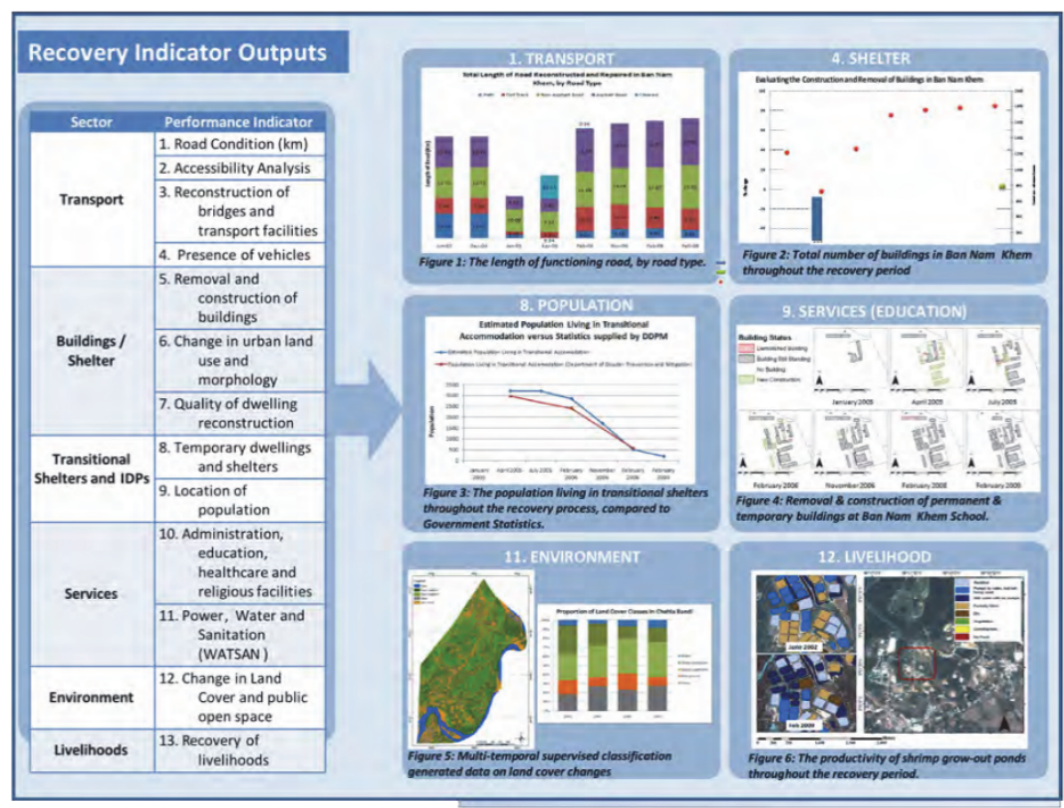

Figure 1.5 Recovery indicator outputs using satellite images. Retrieved from Brown et al. (2010a). 
In a different study, Burton et al. (2011) used repeat photography to evaluate post-Katrina recovery in Mississippi. They took photographs every six months over a three-year period. Then, by assigning scores to each scene in terms of change and recovery, they generated a map for recovery assessment for the entire region. Wagner et al. (2012) used medium resolution images to capture the rate of recovery for post-tornado sites in Oklahoma in 1990. They used remote sensing images to support government and decision-makers by monitoring reconstruction processes, which is reasonable considering the use of medium resolution images and the complexity of urban areas.

Night-time lights satellite images have also been used for damage and recovery analysis. It has been demonstrated that there is a close relation between light intensity and economic activity (Chen and Nordhaus 2011; Sutton et al. 2007). For instance, Gillespie et al. (2014) analyzed responses of night-time light to tsunami damage and recovery in Sumatra. They demonstrated that there are strong relations between brightness values of light images and per capita expenditures and spending on energy and food. Klomp (2016) studied the impact of natural disasters on economic development using satellite night-time light images. In terms of using light images as a proxy for GDP per capita, he showed that natural disasters reduce the amount of lights visible from outer space significantly in the short run, and thus, they lead to a large drop in the luminosity in the developing and emerging market countries. An important limitation of using light satellite images for post-disaster recovery assessment is their low spatial resolution.

Brown et al. (2011) developed a model to assess the damage and early recovery using remote sensing data and ground survey tools after the 2008 Wenchuan earthquake in China. The recovery step of their study includes buildings, accessibility (Figure 1.6), power, and water livelihoods assessments. In this study, only the Normalized Differential Vegetation Index (NDVI) computation was implemented automatically, and other information such as building change detection, accessibility assessments, etc. were done manually. 


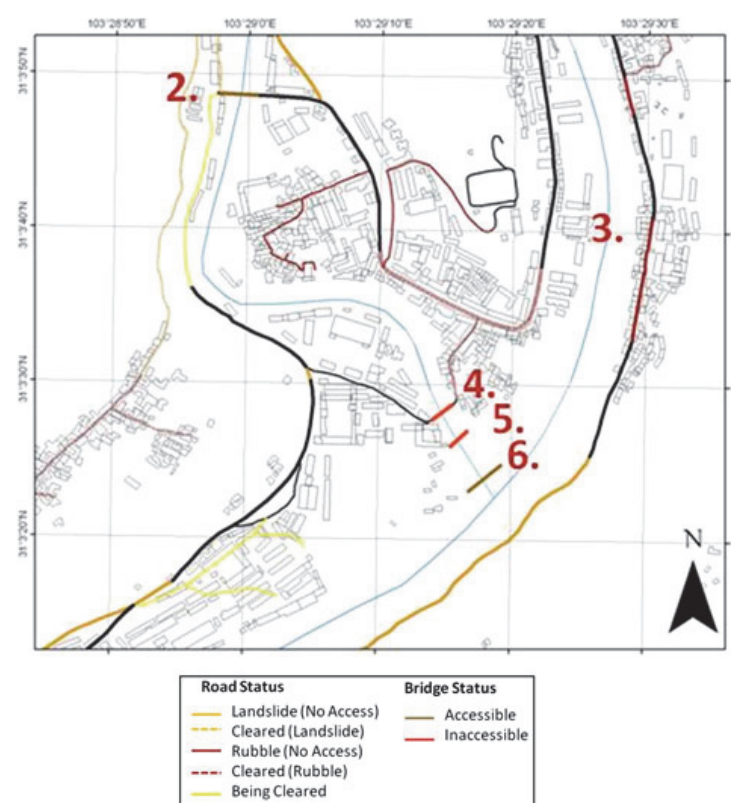

Figure 1.6 Accessibility assessment of roads and bridges. Retrieved from Brown et al. (2011).

Costa Viera and Kerle (2014) studied urban recovery using geospatial data for the firework disaster in Enschede, The Netherlands, 2000. They mainly used building morphology, such as building density, shape and size, and concentration of road networks as indicators. Also, they proposed a proxy to measure the quality of housing based on the energy loss indicator of the buildings (Figure 1.7). Their primary focus was on built-up, and the environmental components of the recovery process and socio-economic aspects were not studied. They also concluded that remote sensing and landscape metrics could provide valuable information about the changes in the landscape and the recovery of functions. 

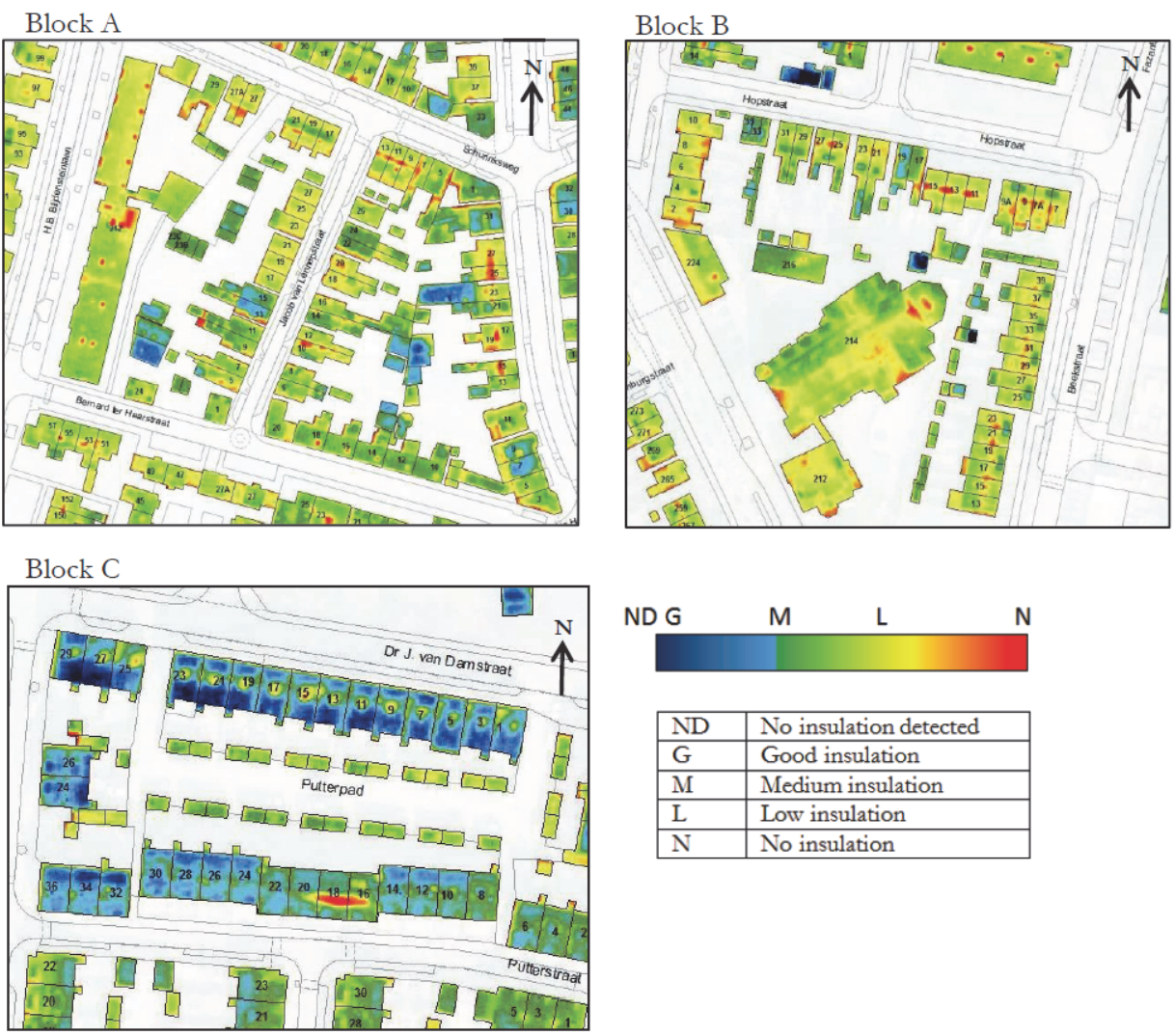

Figure 1.7 Thermal scan images to measure the quality of housing. Retrieved from Costa Viera and Kerle (2014).

On the other hand, disaster resilience in the DRM concept has not been sufficiently studied in the remote sensing field, yet. However, Renschler (2011) concluded that using historical and continuously gathered information through remote sensing and also Geographic Information Systems (GIS) can play a significant role in assessing the resilience of all integrated urban systems and feed a predictive resilience model. Keating et al. (2014) also mentioned that the importance of monitoring a considered region using remote sensing data could be effective in the framework of iterative risk management.

\subsection{Agent-based modeling}

Remote sensing can be used as a recovery monitoring tool, but it cannot explain the results and the reasons for the changes. In addition, in a postdisaster recovery process many variables exist which have impacts on the recovery process, including social networks and an individuals' behavior. However, the effect of each is not known in the recovery process. Therefore, to understand and explore the impacts of these components, simulation of the 
recovery process is needed. Computer-based models such as the Agent-based models ( $A B M)$ allows simulating the recovery process from simple to complex forms, to explain the impact of each aspect on the process. Accordingly, policy and decision-makers can take advantage of the simulation outcomes to improve the process.

In an ABM, each agent (decision-maker) considers its current situation between other agents in the model to decide and act based on defined rules for its behavior in that specific situation (Figure 1.8). In an ABM the initial state of the environment and attributes of agents should be specified by a modeler.

\section{Target System Agent based model}

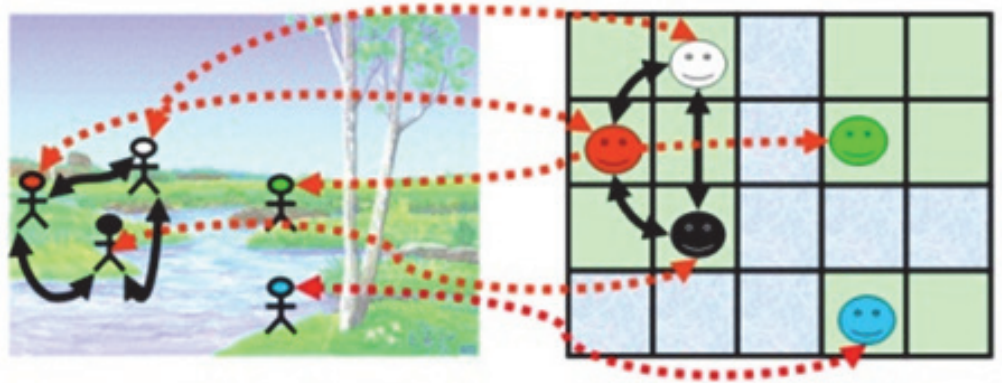

Entities

Agents

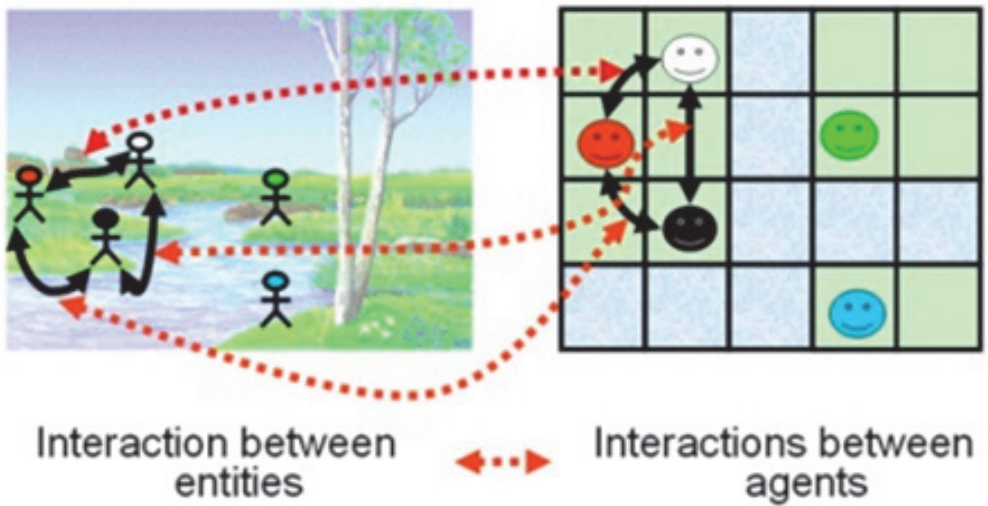

Figure 1.8 Agent-based modeling visualization. Retrieved from Galan et al. (2009).

The attributes of the agents might include internalized behavioral norms, type of characteristics, modes of communication and learning, and internally stored information about itself and other agents (Tesfatsion 2002). All the interactions between agents are tracked during the simulation process to see what happens over time. ABMs can simulate a far wider range of nonlinear behaviour than other conventional models. Therefore, it constitutes an opportunity for policy- 
makers to test different policy scenarios in an artificial simulation environment and explore their consequences (Farmer and Foley 2009).

Several researchers used ABM in the context of disasters (An 2012; Grinberger and Felsenstein 2016), for example for flood incident management (Dawson et al. 2011), tsunami evacuation (Wang et al. 2016), road networks capacity for after disaster evacuation (Chen and Zhan 2008), distribution of aid after a disaster, how rumors relating to aid availability propagate through the population (Crooks and Wise 2013), and dynamics of coastal adaptation for climate risk (Mcnamara and Keeler 2013). They all demonstrated the importance of including human behavior in such a model for accurate simulation outcomes.

Recently the importance of the use of $A B M$ for understanding the recovery processes has been demonstrated (Mishra et al. 2018) and researchers started simulating the post-disaster recovery process (Coates et al. 2019; Fan et al. 2019; Kanno et al. 2018; Nejat and Damnjanovic 2012). However, since recovery is a complex process consisting of various components that may change based on disaster type and environment conditions, the few existing studies are not sufficient to understand and explore all influential factors of the recovery, and consequently, there is a need for ABM-based experiments to explore other components and their effects in the recovery process.

\subsection{Research gap and objectives}

Post-disaster recovery is the least studied component of the DRM cycle, and there is a need for a conceptual framework for post-disaster recovery assessment using remote sensing. In the existing literature most of the remote sensing-based methods for recovery assessment focused on the reconstruction part of the recovery, by using change detection techniques to extract whether damaged buildings were reconstructed or new buildings or structures built. In addition, some researchers studied the recovery process in its long-term phase, utilizing indicators such as the reconstruction of bridges and roads for accessibility analysis, change detection in land cover classification to extract environmental changes, and so forth (Brown et al., 2010). However, the emerging limitation is in the reliability of the defined indicators/proxies. For instance, by extracting green spaces in urban areas, how accurately can the environmental recovery of the area be evaluated? Another significant issue is the recovery of functions in the area, which has not yet been studied. For example, in the post-disaster recovery phase the functionality of the buildings may change, which cannot be identified by only extracting the reconstructed buildings. Similarly, transportation functional recovery analysis cannot be done only by extracting reconstruction of roads and bridges. 
In the literature, most of the remote sensing-based indicators were extracted manually or, in some cases, using semi-automatic image processing methods. Therefore, it becomes a time consuming and tedious process, and clearly demonstrates the need for automatic methods to extract information from remote sensing data. Moreover, conventional methods were used as semiautomatic methods. For example, Brown et al. (2010) used maximum likelihood classification to classify the land cover. This demonstrates the need for automated yet accurate remote sensing data analysis methods to efficiently extract the relevant information. The current state of the art methods, i.e., machine learning and deep learning methods, provide promising accuracy rates in extracting information from remote sensing images. Although they provide accurate results, there is a need for training sample generation to feed in the model to start with initially. Yet, developing a fully automatic machine learning methods is a challenge, which is critical for decreasing the entire process time.

Another issue is the need for high computation power for processing the big remote sensing data (e.g., satellite images). This need even increases by adding the complexity of state-of-the-art methods (e.g., advanced machine learning). Indeed, we need a supercomputer to implement an advanced machine learning method to extract relevant information for several time epochs before and after a disaster to monitor the post-disaster recovery process comprehensively.

Post-disaster recovery monitoring using remote sensing can give valuable information regarding the processes and identify areas that were reconstructed or completely removed after a disaster. However, another important issue for the policy and decision-makers is to find out the reasons for weak and strong recoveries in addition to monitoring the process. Modeling the recovery process using the computer-based simulations such as agent-based modeling allows simulating it from simplest to complex forms, including critical human activities in the society, to understand and explore the impact of each aspect on the process. ABMs have been studied in the response phase of the DRM concept, particularly for modeling evacuation and aid distribution in disasters. However, the utility of ABMs in the DRM context does not stop at this stage; ABMs can also be used to forecast the developments for recovery processes after the event (Crooks and Wise 2013).

Spatial data from standard GIS layers have been employed in ABMs (Heppenstall et al. 2012; Simmonds et al. 2019); however, time series remote sensing data have not been used as the primary source for an ABM, especially in post-disaster recovery and resilience assessments. By increasing spatial, spectral, and temporal resolution of remote sensing data, several types of information can be used in ABMs to increase their efficiency and the accuracy 
of the simulation. This also decreases the dependencies of the ABMs on costly and time-consuming surveys.

\subsection{Research questions}

The aim of this Ph.D. research is to analyze the potential of spatial/remote sensing data to support governments, policy makers, and disaster planners in post-disaster recovery and resilience assessments not only from a physical perspective, but also the socio-economic side. In line with this aim and above mentioned research objectives, six research questions are posed.

1. What are the state-of-the-art remote sensing-based proxies/indicators for disaster risk management and resilience assessment.

2. How to conceptualize post-disaster recovery assessment, including its different types and aspects, based on remote sensing data?

3. How to automate the extraction of useful information from remote sensing data to evaluate the post-disaster damage and recovery process.

4. How to increase the precision and accuracy of remote sensing-based damage and recovery assessments?

5. To what extent cloud computing, i.e., Google Earth Engine, can be used to monitor the post-disaster recovery process?

6. How to integrate multi-temporal remote sensing data with $A B M$ to assist explanation of the different recovery patterns?

\subsection{Structure of the thesis}

This thesis is composed of 8 chapters. Chapter 1 and chapter 8 are introduction and synthesis, respectively. The chapters in-between explain/consist of the leading scientific findings of this study for each specific objective, providing an independent introduction, methods, results and discussion, and conclusions sections. More specifically, the organization of the chapters is as follows:

Chapter 1 - Introduction: introduces and motivates this research, presents the research objectives and overall contributions.

Chapter 2 - Remote sensing-based proxies for urban disaster risk management: A review: provides a comprehensive review of the current remote sensing-based proxies developed for urban disaster risk management. In particular, the proxies are sorted for two risk elements typically associated with pre-disaster situations (vulnerability and resilience), and two postdisaster elements (damage and recovery). The proxies are reviewed in the context of four primary environments and their corresponding sub-categories: built-up (buildings, transport, and others), economic (macro, regional and urban economics, and logistics), social (services and infrastructures, and socioeconomic status), and natural. All environments and the corresponding proxies 
are discussed and analyzed in terms of their reliability and sufficiency in comprehensively addressing the selected DRM assessments. We highlight the strength and identify gaps and limitations in current proxies, including inconsistencies in terminology for indirect measurements. We present a systematic overview for each group of the reviewed proxies that could simplify cross-fertilization across different DRM domains and may assist the further development of methods. While systemizing examples from the wider remote sensing domain and insights from social and economic sciences, we suggest a direction for developing new proxies, also potentially suitable for capturing functional recovery.

Chapter 3 - A conceptual framework for post-disaster recovery assessment with remote sensing: presents a comprehensive theoretical scheme to monitor and evaluate the post-disaster recovery process and resilience using remote sensing data. In particular, available remote sensing image-based proxies are used to evaluate the recovery addressing, not-only physical but also functional aspects. In addition, this conceptual framework can be used to evaluate disaster resilience assuming that the speed of the recovery is a proxy for resilience assessment. The proxies are mostly extracted using machine learning-derived land cover and land use maps. The proposed approach is used to assess the recovery of barangays (municipalities), including Tacloban city, in the Leyte region in the central Philippines.

Chapter 4 - Towards post-disaster debris identification for precise damage and recovery assessments from UAV and satellite images: discusses the limitations of using debris and rubble piles as proxies for damage detection and subsequent post-disaster recovery assessment from remote sensing images, and investigates two different approaches for post-disaster debris identification. Distinguishing the structural rubble from ephemeral debris can increase the accuracy of the damage and recovery assessments since most of the damage detection methods using this debris as a proxy for damage assessment. Three feature extraction methods i.e., Gabor filters, Local Binary Pattern (LBP), and Histogram of the Oriented Gradients (HOG) are investigated to identify the debris from UAV images. As the second strategy, an approach is proposed, which monitors the multi-temporal satellite images acquired days and weeks after the disaster to figure out the relation between debris type and their time of removal. The approaches are tested for Tacloban city using UAV and multi-temporal satellite images.

Chapter 5 - Post-disaster building database updating using
automated deep learning: An integration of pre-disaster
OpenstreetMap and multi-temporal satellite data: presents an
automated deep learning method of building database updating for post-
disaster damage and recovery assessments. The location of the damaged,


reconstructed, and newly constructed buildings provide critical supporting information for both first responders and recovery planners after a disaster. The proposed method makes use of free OpenStreetMap building footprints available for a pre-disaster situation to automatically collect training areas from very-high-resolution satellite images for a convolutional neural network (i.e., U-net), which is supported with residual connections. The trained network is then transferred and retrained for the post-disaster situation at any time after a simple building-based change detection analysis over OSM data. The proposed approach is tested for different scenarios of damage and recovery assessments in very high-resolution satellite images selected from Tacloban, the Philippines, after Typhoon Haiyan.

Chapter 6 - Post-disaster recovery monitoring with Google Earth Engine: presents a cloud computing-based tool for post-disaster recovery assessment. In previous chapters, computationally expensive methods are developed to extract information from the costly very high-resolution satellite images using paid supercomputers. However, the aim of this chapter is to propose and investigate a completely free tool to monitor the recovery process. Hence, an approach is proposed, which utilizes Google Earth Engine (GEE) as a cloud computing platform and its coding environment, to perform land cover/use classification for different time steps after a disaster. The Random Forest method, which is available in the GEE, is employed as the main method to classify the composite cloud-free Landsat 7 and 8 images. The composite images are generated based on cloud and shadow detection/removal, and computing the mode of the missing pixel values from the collection of the images for the selected time-steps.

Chapter 7 - Agent-based modeling of post-disaster recovery with remote sensing data: introduces the proposed agent-based model, which uses information that was extracted from remote sensing images for postdisaster recovery. The developed post-disaster recovery (PDR) model can be used by decision-makers to understand the recovery process and carry out the most influential factors and components. The satisfaction of the formal building and slum households is tracked and mapped to understand and demonstrate each of which recovery patterns. Also, the effect of the unemployment rate and presence of a relocation site far from urban areas and workplaces after a disaster are experimented using the PDR model.

Chapter 8 - Synthesis: synthesizes the results of the individual chapters. It provides the main findings, contributions of this research, reflects on the work, and discuss the usability of the proposed approaches from a stakeholder perspective. The future outlook for improving each of the proposed research lines and methods in this research is also reported. 
Chapters 2 through 7 are based on the published journal and conference articles. There may, therefore, be repetitive information in the introduction sections of the various chapters. Nevertheless, this makes every chapter standalone and enables them to be considered individually, providing comprehensive information for the readers who are interested in specific chapters.

\subsection{References of Chapter 1}

An, L., 2012. Modeling human decisions in coupled human and natural systems: Review of agent-based models. Ecological Modelling, 229, 2536.

Bakkensen, L. A., Fox-Lent, C., Read, L. K., Linkov, I., 2016. Validating resilience and vulnerability indices in the context of natural disasters. Risk Analysis, 37(5), 982-1004.

Bell, J., Gebremichael, E., Molthan, A., Schultz, L., Meyer, F., Shrestha, S., 2019. Synthetic Aperture Radar and optical remote sensing of crop damage attributed to severe weather in the central United States. Paper presented at the IGARSS 2019, Yokohama, Japan, .

Brown, D., Platt, S., Bevington, J., Saito, K., Adams, B., Chenvidyakarn, T., Spence, R., Chuenpagdee, R., Khan, A., 2015. Monitoring and evaluating post-disaster recovery using high-resolution satellite imagery - towards standardised indicators for post-disaster recovery. University of Cambridge: Cambridge, UK.

Brown, D., Platt, S., Bevington, J., 2010a. Disaster recovery indicators: Guidlines for monitoring and evaluation. University of Cambridge: Cambridge, UK: CURBE, Cambridge University for Risk in the Built Environment, University of Cambridge: Cambridge, UK.

Brown, D., Saito, K., Liu, M., Spence, R., So, E., Ramage, M., 2011. The use of remotely sensed data and ground survey tools to assess damage and monitor early recovery following the 12.5.2008 Wenchuan earthquake in China. Bulletin of Earthquake Engineering, 10(3), 741-764.

Brown, D., Saito, K., Spence, R., Chenvidyakarn, T., 2008. Indicators for measuring, monitoring and evaluating post-disaster recovery. Paper presented at the 6th International Workshop on Remote Sensing for Disaster Applications, University of Cambridge: Cambridge, UK..

Brown, D., Saito, K., T., C., 2010b. Monitoring and evaluating post-disaster recovery using high-resolution satellite imagery, Cambridge University for Risk in the Built Environment, University of Cambridge: Cambridge, UK, 2010.

Bruneau, M., Chang, S. E., Eguchi, R. T., Lee, G. C., O'rourke, T. D., Reinhorn, A. M., Shinozuka, M., Tierney, K., Wallace, W. A., Von Winterfeldt, D., 2003. A Framework to Quantitatively Assess and Enhance the Seismic Resilience of Communities. Earthquake Spectra, 19(4), 733-752.

Burton, C., Mitchell, J. T., Cutter, S. L., 2011. Evaluating post-Katrina recovery in Mississippi using repeat photography. Disasters, 35(3), 488-509.

Burton, C. G., 2014. A validation of metrics for community resilience to natural hazards and disasters using the recovery from hurricane Katrina as a case study. Annals of the Association of American Geographers, 105(1), 67-86. 
Burton, I., Huq, S., Lim, B., Pilifosova, O., Schipper, E. L., 2002. From impacts assessment to adaptation priorities: the shaping of adaptation policy. Climate Policy, 2(2), 145-159.

Chen, S.-W., Sato, M., 2013. Tsunami damage investigation of built-up areas using multitemporal spaceborne full polarimetric SAR images. IEEE Transactions on Geoscience and Remote Sensing, 51(4), 1985-1997.

Chen, X., Nordhaus, W. D., 2011. Using luminosity data as a proxy for economic statistics. Proceedings of the National Academy of Sciences, 201017031.

Chen, X., Zhan, F. B., 2008. Agent-based modeling and simulation of urban evacuation: Relative effectiveness of simultaneous and staged evacuation strategies. Journal of the Operational Research Society, 59(1), 25-33.

Coates, G., Li, C., Ahilan, S., Wright, N., Alharbi, M., 2019. Agent-based modeling and simulation to assess flood preparedness and recovery of manufacturing small and medium-sized enterprises. Engineering Applications of Artificial Intelligence, 78, 195-217.

Cole, M. A., Elliott, R. J. R., Toshihiro, O., Strobl, E., 2013. Natural disasters and plant survival: The impact of the Kobe earthquake. RIETI Discussion Paper Series.

Coppola, D. P., 2015. The Management of Disasters. 3rd ed.; ButterworthHeinemann: Boston, MA, USA, 2015; pp. 1-39.

Costa Viera, A., Kerle, N., 2014. Utility of geo-informatics for disaster risk management: Linking structural damage assessment, recovery and resilience. University of Twente: Enschede, The Netherlands.

Council, N. R., 2012. Disaster resilience: A national imperative. Washington, DC: The National Academies Press.

CRED. 2018. Economic losses, poverty and disasters 1998-2017. Retrieved from CRED:

Crooks, A. T., Wise, S., 2013. GIS and agent-based models for humanitarian assistance. Computers, Environment and Urban Systems, 41, 100-111.

Curtis, A., Duval-Diop, D., Novak, J., 2010. Identifying spatial patterns of recovery and abandonment in the post-Katrina holy cross neighborhood of New Orleans. Cartography and Geographic Information Science, 37(1), 45-56.

Cutter, S. L., Barnes, L., Berry, M., Burton, C., Evans, E., Tate, E., Webb, J., 2008. A place-based model for understanding community resilience to natural disasters. Global Environmental Change, 18(4), 598-606.

Cutter, S. L., Burton, C. G., Emrich, C. T., 2010. Disaster Resilience Indicators for Benchmarking Baseline Conditions. Journal of Homeland Security and Emergency Management, 7(1).

Dadhich, G., Miyazaki, H., Babel, M., 2019. Applications of Sentinel-1 Synthetic Aperture Radar imagery for floods damage assessment: A case study of Nakhon Si Thammarat, Thailand. ISPRS - International Archives of the Photogrammetry, Remote Sensing and Spatial Information Sciences, 4213, 1927-1931.

Dalati, M., 2005, 9-11 June 2005. Remote sensing techniques in active faults surveying. Case study: detecting active faulting zones NW of Damascus, Syria. Paper presented at the Proceedings of 2nd International Conference on Recent Advances in Space Technologies, 2005. RAST 2005.

Dawson, R. J., Peppe, R., Wang, M., 2011. An agent-based model for riskbased flood incident management. Natural Hazards, 59(1), 167-189. 
Duarte, D., Nex, F., Kerle, N., Vosselman, G., 2018a. Multi-resolution feature fusion for image classification of building damages with convolutional neural networks. Remote Sensing, 10(10), 1636.

Duarte, D., Nex, F., Kerle, N., Vosselman, G., 2018b. Satellite image classification of building damages using airborne and satellite image samples in a deep learning approach. ISPRS Annals of Photogrammetry, Remote Sensing and Spatial Information Sciences, IV-2, 89-96.

Fan, C., Gong, L., Li, H., 2019. An agent-based model approach for assessing tourist recovery strategies after an earthquake: A case study of Jiuzhai Valley. Tourism Management, 75, 307-317.

Farmer, J. D., Foley, D., 2009. The economy needs agent-based modelling. Nature, 460, 685-686.

Fisher, R. E., Bassett, G. W., Buehring, W. A., Collins, M. J., Dickinson, D. C., Eaton, L. K., Haffenden, R. A., Hussar, N. E., Klett, M. S., Lawlor, M. A., Millier, D. J., Petit, F. D., Peyton, S. M., Wallace, K. E., Whitfield, R. G., Peerenboom, J. P., 2010. Constructing a resilience index for the enhanced critical infrastructure protection program (ANL/DIS-10-9; TRN: United States 10.2172/991101. Retrieved from https://www.osti.gov/servlets/purl/991101

Galarreta, J. F., Kerle, N., Gerke, M., 2015. UAV-based urban structural damage assessment using object-based image analysis and semantic reasoning. Natural Hazards and Earth System Sciences, 15(6), 10871101.

Gillespie, T. W., Frankenberg, E., Chum, K. F., Thomas, D., 2014. Night-time lights time series of tsunami damage, recovery, and economic metrics in Sumatra, Indonesia. Remote Sensing Letters, 5(3), 286-294.

Grinberger, A. Y., Felsenstein, D., 2016. Dynamic agent based simulation of welfare effects of urban disasters. Computers, Environment and Urban Systems, 59, 129-141.

Haas, J. E., Kates, R. W., Bowden, M. J., 1977. Reconstruction following disaster. Cambridge, MA: The MIT Press.

Hallegatte, S., Bangalore, M., Vogt-Schilb, A., 2016. Assessing Socioeconomic Resilience to Floods in 90 Countries. Retrieved from World Bank Group.

Heppenstall, A., Crooks, A., See, L. M., Batty, M., 2012. Agent-based models of geographical systems: Springer Netherlands, VIII, 760.

Holling, C. S., 1973. Resilience and stability of ecological systems. Annual Review of Ecology and Systematics, 4(1), 1-23.

IFRC, 2016. What is vulnerability?. Retrieved from: https://www.ifrc.org/en/what-we-do/disaster-management/aboutdisasters/what-is-a-disaster/what-is-vulnerabilit.

Jones, L., Ballon, P., 2020. Tracking changes in resilience and recovery after natural hazards: Insights from a high-frequency mobile-phone panel survey. Global Environmental Change, 62, 102053.

Jordan, E., Javernick-Will, A., Amadei, B., 2011. Pathways to communicate recovery and resiliency. Paper presented at the Engineering Project Organizations Conference, Estes Park, Colorado.

Joyce, K. E., Wright, K. C., Samsonov, S. V., Ambrosia, V. G., 2009. Remote sensing and the disaster management cycle. doi: 10.5772/8341.

Kanno, T., Koike, S., Suzuki, T., Furuta, K., 2018. Human-centered modeling framework of multiple interdependency in urban systems for simulation of 
post-disaster recovery processes. Cognition, Technology \& Work, 21(2), 301-316.

Kates, R. W., Colten, C. E., Laska, S., Leatherman, S. P., 2006. Reconstruction of New Orleans after Hurricane Katrina: A research perspective. Proceedings of the National Academy of Sciences, 103(40), 14653-14660.

Keating, A., Campbell, K., Mechler, R., Michel-Kerjan, E., Mochizuki, J., Kunreuther, K., Bayer, J., Hanger, S., Mccallum, I., See, L., Williges, K., Atreya, A., Wouter Botzen, Collier, B., Czajkowski, J., Hochrainer, S., C., E., 2014. Operationalizing resilience against natural disaster risk: Opportunities, barriers, and a way forward. Retrieved from Zurich Flood Resilience Alliance.:

Kerle, N., 2011. Remote sensing based post-disaster damage mapping, ready for a collaborative approach. Earthzine. Retrieved from: https://earthzine.org/remote-sensing-based-post-disaster-damagemapping-ready-for-a-collaborative-approach.

Kerle, N., Hoffman, R. R., 2013. Collaborative damage mapping for emergency response: The role of cognitive systems engineering. Natural Hazards and Earth System Science, 13(1), 97-113.

Kerle, N., Nex, F., Gerke, M., Duarte, D., Vetrivel, A., 2019. UAV-based structural damage mapping: A review. ISPRS International Journal of GeoInformation, 9(1), 14.

Klemas, V., 2014. Remote sensing of floods and flood-prone areas: An overview. Journal of Coastal Research, 31(4), 1005-1013.

Klomp, J., 2016. Economic development and natural disasters: A satellite data analysis. Global Environmental Change, 36, 67-88.

Liu, Z., Min, M., Li, J., Sun, F., Di, D., Ai, Y., Li, Z., Qin, D., Li, G., Lin, Y., Zhang, X., 2019. Local severe storm tracking and warning in preconvection stage from the new generation geostationary weather satellite measurements. Remote Sensing, 11(4), 383.

Lum, T., Margesson, R., 2014. Typhoon Haiyan (Yolanda): U.S. and international response to Philippines disaster. Retrieved from: https://fas.org/sgp/crs/row/R43309.pdf.

Maclean, K., Cuthill, M., Ross, H., 2014. Six attributes of social resilience. Journal of Environmental Planning and Management, 57(1), 144-156.

Manyena, S. B., 2006. The concept of resilience revisited. Disasters, 30(4), 433-450.

Mayunga, J. S., 2007. Understanding and applying the concept of community disaster resilience: A capital-based approach, USA. https://doi.org/10.1146/annurev.energy.32.051807.090348.

Mcnamara, D. E., Keeler, A., 2013. A coupled physical and economic model of the response of coastal real estate to climate risk. Nature Climate Change, 3, 559-562.

Mishra, D., Kumar, S., Hassini, E., 2018. Current trends in disaster management simulation modelling research. Annals of Operations Research, 283(1-2), 1387-1411.

Nashwan, M. S., Shahid, S., Wang, X., 2019. Assessment of satellite-based precipitation measurement products over the hot desert climate of Egypt. Remote Sensing, 11(5), 555.

Nejat, A., Damnjanovic, I., 2012. Agent-based modeling of behavioral housing recovery following disasters. Computer-Aided Civil and Infrastructure Engineering, 27(10), 748-763. 
Nex, F., Duarte, D., Steenbeek, A., Kerle, N., 2019. Towards real-time building damage mapping with low-cost UAV solutions. Remote Sensing, 11(3), 287.

Norris, F. H., Stevens, S. P., Pfefferbaum, B., Wyche, K. F., Pfefferbaum, R. L., 2008. Community resilience as a metaphor, theory, set of capacities, and strategy for disaster readiness. Am J Community Psychol, 41(1-2), 127150.

Olshansky, R. B., Johnson, L. A., Topping, K. C., 2006. Rebuilding communities following disaster: Lessons from Kobe and Los Angeles. Built Environment (1978-), 32(4), 354-374.

Platt, S., Brown, D., Hughes, M., 2016. Measuring resilience and recovery. International Journal of Disaster Risk Reduction, 19, 447-460.

Pradhan, B., Singh, R. P., Buchroithner, M. F., 2006. Estimation of stress and its use in evaluation of landslide prone regions using remote sensing data. Advances in Space Research, 37(4), 698-709.

Rastiveis, H., Eslamizade, F., Hosseini-Zirdoo, E., 2015. Building damage assessment after earthquake using post-event LiDAR data. ISPRS International Archives of the Photogrammetry, Remote Sensing and Spatial Information Sciences, XL-1-W5, 595-600.

Renschler, C. S., Frazier, A. E., Arendt, L., Cimellaro, G. P., reinhorn, A. M., Bruneau, M., 2011. A framework for defining and measuring extreme events resilience at the community scale: the PEOPLES resilience framework. MCEER-10-0006, Earthquake Engineering to Extreme Events.

Revet, S., 2012. Conceptualizing and confronting disasters: A panorama of social science research and international policies. In F. Attinà (Ed.), The Politics and Policies of Relief, Aid and Reconstruction: Contrasting Approaches to Disasters and Emergencies (pp. 42-56). London: Palgrave Macmillan UK.

Rose, A., 2009. Economic resilience to disasters. in B. Ayyub (ed.), Economics of Community Disaster Resilience, Gaithersburg, MD: National Institute of Standards and Technology.

Rose, A., Krausmann, E., 2013. An economic framework for the development of a resilience index for business recovery. International Journal of Disaster Risk Reduction, 5, 73-83.

Sadiq, A.-A., Tyler, J., Noonan, D. S., 2019. A review of community flood risk management studies in the United States. International Journal of Disaster Risk Reduction, 41, 101327.

Sharifi, A., Yamagata, Y., 2016. On the suitability of assessment tools for guiding communities towards disaster resilience. International Journal of Disaster Risk Reduction, 18, 115-124.

Simmonds, J., Gómez, J. A., Ledezma, A., 2019. The role of agent-based modeling and multi-agent systems in flood-based hydrological problems: A brief review. Journal of Water and Climate Change, jwc2019108. doi: https://doi.org/10.2166/wcc.2019.108.

Sutton, P., Elvidge, C., Ghosh, T., 2007. Estimation of gross domestic product at sub-national scales using night-time satellite imagery. Int. J. Ecol. Econ. Stat., 8, 5-21.

Tesfatsion, L., 2002. Economic agents and markets as emergent phenomena. Proc Natl Acad Sci USA, 99 Suppl 3, 7191-7192. 
Thies, B., Bendix, J., 2011. Satellite based remote sensing of weather and climate: Recent achievements and future perspectives. Meteorological Applications, 18(3), 262-295.

Tiernan, A., Drennan, L., Nalau, J., Onyango, E., Morrissey, L., Mackey, B., 2019. A review of themes in disaster resilience literature and international practice since 2012. Policy Design and Practice, 2(1), 53-74.

Timmerman, P., 1981. Vulnerability, resilience and the collapse of society: $A$ review of models and possible climatic applications. Toronto, Canada: Institute for Environmental Studies, University of Toronto.

Townshend, I., Awosoga, O., Kulig, J., Fan, H., 2014. Social cohesion and resilience across communities that have experienced a disaster. Natural Hazards, 76(2), 913-938.

UNDP. 1993. Rehabilitation and reconstruction. Retrieved from: https://opendocs.ids.ac.uk/opendocs/bitstream/handle/20.500.12413/5 659/rg233.pdf; sequence $=1$.

UNISDR. 2009. Terminology on disaster risk reduction. United Nations International Strategy for Disaster Reduction (UNISDR), Geneva, Switzerland.

UNISDR. 2015, 14-18 March. Sendai framework for disaster risk reduction 2015 - 2030. Paper presented at the Third World Conf. Disaster Risk Reduction, Sendai, Japan.

Vetrivel, A., Gerke, M., Kerle, N., Vosselman, G., 2016a. Identification of structurally damaged areas in airborne oblique images using a visual-bagof-words approach. Remote Sensing, 8(3), 231.

Vetrivel, A., Kerle, N., Gerke, M., Nex, F., Vosselman, G., 2016b. Towards automated satellite image segmentation and classification for assessing disaster damage using data specific features with incremental learning. Paper presented at the GEOBIA 2016, Enschede, The Netherlands.

Wagner, M. A., Myint, S. W., Cerveny, R. S., 2012. Geospatial assessment of recovery rates following a tornado disaster. IEEE Transactions on Geoscience and Remote Sensing, 50(11), 4313-4322.

Wang, H., Mostafizi, A., Cramer, L. A., Cox, D., Park, H., 2016. An agent-based model of a multimodal near-field tsunami evacuation: Decision-making and life safety. Transportation Research Part C: Emerging Technologies, 64, 86-100.

Yulianto, F., Sofan, P., Zubaidah, A., Sukowati, K. a. D., Pasaribu, J. M., Khomarudin, M. R., 2015. Detecting areas affected by flood using multitemporal ALOS PALSAR remotely sensed data in Karawang, West Java, Indonesia. Natural Hazards, 77(2), 959-985. 


\section{Chapter 2 - $\quad$ Remote sensing-based proxies for urban disaster risk management: $\mathrm{A}_{\text {review }}{ }^{1}$}

1 This chapter is based on:

- Ghaffarian, S., Kerle, N., Filatova, T., 2018. Remote sensing-based proxies for urban disaster risk management and resilience: A review. Remote Sens., 10, 1760.

- Ghaffarian, S., Kerle, N., Filatova, T., 2018. A review of remote sensing-based proxies and data processing methods for urban disaster risk management. Presented at the EGU general assembly, Vienna, Austria, 8-13 April, 2018. 


\begin{abstract}
Rapid increase in population and growing concentration of capital in urban areas has escalated both the severity and longer-term impact of natural disasters. As a result, Disaster Risk Management (DRM) and reduction have been gaining increasing importance for urban areas. Remote sensing plays a key role in providing information for urban DRM analysis due to its agile data acquisition, synoptic perspective, growing range of data types, and instrument sophistication, as well as low cost. As a consequence numerous methods have been developed to extract information for various phases of DRM analysis. However, given the diverse information needs, only few of the parameters of interest are extracted directly, while the majority have to be elicited indirectly using proxies. This paper provides a comprehensive review of the proxies developed for two risk elements typically associated with pre-disaster situations (vulnerability and resilience), and two post-disaster elements (damage and recovery), while focusing on urban DRM. The proxies were reviewed in the context of four main environments and their corresponding sub-categories: built-up (buildings, transport, and others), economic (macro, regional and urban economics, and logistics), social (services and infrastructures, and socio-economic status), and natural. All environments and the corresponding proxies are discussed and analyzed in terms of their reliability and sufficiency in comprehensively addressing the selected DRM assessments. We highlight strength and identify gaps and limitations in current proxies, including inconsistencies in terminology for indirect measurements. We present a systematic overview for each group of the reviewed proxies that could simplify cross-fertilization across different DRM domains and may assist the further development of methods. While systemizing examples from the wider remote sensing domain and insights from social and economic sciences, we suggest a direction for developing new proxies, also potentially suitable for capturing functional recovery.
\end{abstract}




\subsection{Introduction}

By $205080 \%$ of the world population will live in urban areas (United Nations 2015). This unprecedented clustering of infrastructure and people has been shifting the focus of Disaster Risk Management (DRM) studies towards cities. Furthermore, the ability of a system to resist, absorb, accommodate to and recover from the effects of a hazard in a timely and efficient manner - knows as resilience - has become crucial to decrease the disaster risk (UNISDR 2009). Resilience also encompasses post-event processes that allow communities to reorganize, change, and learn in response to an event (Cutter et al. 2008). Hence, enhancing community's resilience to natural hazards implies improving its capacity to anticipate threats, to reduce own overall vulnerability, and to allow the community to recover from adverse impacts when they occur. Decades of disaster research offer extensive findings in this respect (Burton 2014; Cutter et al. 2016; Cutter et al. 2010; Norris et al. 2008). Remote sensing (RS) as an effective and rapid tool for monitoring large areas- is essential for the acquisition of geospatial data, which in turn constitutes the basis for risk assessment and management. RS is widely used for various aspects of the DRM, ranging from vulnerability (Taubenböck et al. 2008) to rapid damage assessments (Cooner et al. 2016), for diverse areas ranging from coastal ecosystems (Mccarthy et al. 2017) to complex urban settings (Du et al. 2014), and for disasters as diverse as landslides (Stumpf and Kerle 2011; Zhao and Lu 2018) or cyclones (Hoque et al. 2017).

Numerous methods have been developed to extract information from RS data to identify, characterize or quantify different phases of the disaster risk cycle: response, recovery, prevention/mitigation and preparedness (Coppola 2015). However, early studies predominantly considered the physical side of the assessments for both pre- and post-disaster phases and hazard assessment, using direct observations. For example, scholars have assessed the number of buildings collapsed/damaged (Arciniegas et al. 2007; Saito et al. 2004) and reconstructed (Berke et al. 1993), or estimated their vulnerability (Mueller et al. 2006). However, the attention to the non-physical side of DRM in both preand post-disaster situations was scare. Non-physical assessments usually comprise social, economic and natural aspects in addition to the built-up ones, which refer to physical assessments, e.g., physical vulnerability assessment (MCDEM 2005). Recent studies started to include socio-economic aspects when examining DRM phases such as vulnerability (Flax et al. 2002; Morrow 1999), resilience (Burton 2012), damage (Bevington et al. 2010; Bradshow 2004), and recovery (Rubin et al. 1985) assessments. While several spatial and nonspatial parameters required for detecting and quantifying of DRM-related elements can be extracted directly from RS imagery, many have to be elicited indirectly. Similarly, Indirect measurements are fundamental tools in related research fields, such as environmental science (Pidwirny 2017). They have been also used in social and economic studies in the DRM context, e.g. for 
social vulnerability (Fatemi et al. 2017) and business recovery (Rose and Krausmann 2013) assessments. Proxies have become central in RS-based DRM studies due to the inherent characteristics of several urban elements such as social and economic activities, which makes it very difficult, if not impossible, to directly observe them using RS data. Proxies use observable features in RS data used to capture and extract information of interest that is not directly visible or measurable but is correlated with the former. In recent years, using proxies has become the predominant approach to capture implicit information in urban DRM, for both pre- and post-disaster situations (Taubenböck et al. 2009). For example, texture is used as a proxy to extract damage to buildings and roads, with irregular texture indicating damaged area. For the purpose of this review, the most relevant components of the disaster risk cycle for use of RS in urban areas are the post-disaster phase proxies focusing on damage and recovery, and the pre-disaster phase proxies measuring vulnerability and resilience.

This work was specifically motivated by a number of limitations in the published literature. Firstly, there is inconsistency in terminology: indirect measurements labelled by various terms such as index (Kotzee and Reyers 2016), indicator (Platt et al. 2016), and proxy (Ebert et al. 2009). In this paper, the term proxy is overarching for all such indirect measurements. Secondly, duplications in efforts: in certain cases methodologically the same proxies have been developed independently in more than one domain. For instance, the presence of vegetation in urban areas has been used as a proxy for both social vulnerability (Ebert et al. 2009) and post-disaster recovery (Brown et al. 2010a) assessments. Thirdly, the current DRM literature suggests several proxies that are at times unreliable. For example, the presence of vehicles has been used to evaluate the accessibility of the roads (Brown et al. 2010a). However, the metric is highly dependent on the image acquisition date and time, and other legal or environmental parameters that affect the presence of vehicles. Finally, need to go beyond the physical side towards functional assessments is prominent. Yet, few proxies have been developed for social, economic and functional assessments in urban DRM. This paper conducts critical analyses on current RS-based proxies and borrows insights from other research fields (e.g., the economy and social sciences). To address these limitations, we conduct a systematical review following a number of steps. We start by splitting reviewed proxies into four groups that characterize built-up, economic, social, and natural environments. Then, a proxy catalog for each group is generated based on the reviewed studies used by researchers and governments in the different DRM phases. Finally, a comprehensive analysis is done on the reviewed proxies, and the most suitable proxies for each DRM situation are discussed. These analysis demonstrates current limitations including lack of proxies to evaluate urban functions for DRM and suggests future directions in developing new proxies. Furthermore, proxies originating 
from the wider RS domain and other disciplines - urban form studies, structural engineering, and natural sciences - were identified and systemized in this paper. This structured analysis may provide guidance for developing reliable RS-based proxies.

The review is structured as follows. Section 2 defines the terminology used for indirect measurements, i.e. proxy, in this article. In Section 3 we explain the methodology employed for this review. Section 4 presents a comprehensive literature review of RS-based proxies for each of the four selected environments, as well as a corresponding table of proxies. In addition, new insights towards improving current or developing new proxies are provided based on the state of the art analysis from different research fields including urban form studies, structural engineering, economics and natural sciences. The final section provides conclusions and outlines directions for future development of the field.

\subsection{Defining a proxy in remote sensing}

Indirect estimation using proxies allows researchers to deduce the condition of an element or process based on their relations and links with directly identifiable and observable features in the surrounding/neighboring areas. According to the Oxford Dictionary, a proxy is a figure that can be used to represent the value of a non-directly measurable object in a calculation. In statistics, it is a measurable variable that is used in place of a variable that cannot be measured (Upton and Cook 2014). Moreover, there are good examples of using proxies in economics, such as per capita Gross Domestic Product (GDP) being a proxy for growth in wealth and potentially quality of life (Montgomery et al. 2000).

There is currently no clear definition for proxy in RS-based studies, though proxies play a critical role in this field. Considering the growing role of proxies in RS, similarities and differences can be found when compared with the traditional use of proxies, particularly in the wider geosciences (including climatology). In all disciplines, proxies have been used as indirect variables to determine or constrain unobservable or immeasurable variables or as physical variables to go back in time and measure the immeasurable or unobservable parameters because of lack of data from that time. However, passive RS is by definition an indirect form of measurement and an information source that acquires data at a certain time. By that logic any image derivative or index measure is a proxy. Therefore, the traditional definition of the proxy cannot be used in this field. In this paper, the term proxy is defined as use of observable physical features or directly measurable variables to understand and extract what actually exists on the ground, but what is not directly observable or measurable from RS data. For example, the proportion of built-up and 
vegetated area has been measured and used as a proxy to determine a settlement type to determine the vulnerability of such settlements to disasters (Ebert et al. 2009). Proxies can also be separated into two groups based on their use in the RS field:

(i) not-directly observable physical parameters, e.g. use of shadow to determine the height of an element at risk (building) (Contreras et al. 2016; Kerle and Hoffman 2013),

(ii) and measures to assess processes and functions, e.g. the building morphology and use to determine functionality of buildings and urban areas to assess the post-disaster recovery processes and vulnerability (Liu et al. 2017a).

\subsection{Methods}

We systemize the published literature based on classification by (i) DRM stage (focus on pre- and post-disaster) zooming into vulnerability, resilience, damage and recovery assessments practices, and by (ii) the type of environment changes in which a proxy tries to capture. Each is common in DRM literature and fits well the main purpose of this article. Following (Mcdem 2005), we differentiate between 4 types of environment: (1) the built-up environment divided into 'buildings', 'transport' and 'others'; (2) the economic environment with 'macro, regional and urban economics' and 'logistics' subcategories; (3) the social environment covering 'services and infrastructure' and 'socio-economic status'; and (4) the natural environment.

This review is based on a systematic literature search, performed in summer 2018, using several databases (Web of Science, ScienceDirect, SpringerLinkjournals, Taylor\&Francis and Scopus). We select the articles for this review, using the following keywords: indirect, proxy, proxies, index, indices, indicator, and remote sensing, GIS, vector, raster, map, satellite image, aerial image, lidar, UAV, UAS, drone, crowdsourced, ground image, ground photo, and resilience, resilient, adaptive capacity, coping capacity, preparation, prevention, vulnerability, damage, response, impact, rescue, recovery, reconstruction, rehabilitation and relief. These keywords are selected to address the goal of the review: to provide a systematic categorization of proxies for DRM and resilience, supporting them with some key citations and relevant state-of-the-art examples. Finding relevant papers for this review was difficult because not necessarily all of the papers use keywords to describe their indirect measurements (proxies). Indeed, with nearly every type of passive RS being per se indirect, every form of processing of such data inherently uses proxies. Furthermore, there are many commonly used proxies (e.g., NDVI for vegetation cover extraction, the number or configuration of buildings to extract information about urban sprawl and presence of slums, 
nightlights to detect electrification) that researchers accept as natural RSbased measurements, without labeling them as proxies. Hence, there may be additional studies using RS-based proxies that are not included in this review due to different terminology. Moreover, there might be uncertainties in the used terms for the selected pre-and post-disaster situations, which also made it hard to find relevant studies. For example, for post-disaster damage assessment, similar key phrases have been used such as response to a disaster (Gil and Steinbach 2008), and impacts of a disaster (Mohan and Strobl 2017). Naturally, to the best of our knowledge we have carried the search as comprehensively as possible. Since the purpose of this article is to provide a structured overview of existing approaches with exemplary citations on key studies rather than perform a full coverage of the literature, the core conclusions are not affected. The review covered journal publications, book sections and conference publications that can be retrieved either via the research engines employed or the websites of the main RS conferences. Only English language papers were considered.

\subsection{Remote sensing-based proxies for DRM in urban areas}

In total, we identify 114 key publications. Out of these, 52 papers address damage assessment and 21, 40, and seven articles focus on recovery, vulnerability, and resilience assessments, respectively. Papers with a focus on two DRM areas, e.g., both damage and recovery assessments are counted on both of them separately. A chronological overview of the publications shows an increasing trend in studies for RS-based proxy literature for urban DRM (Figure 2.1). Continuous progress in RS technology and sensors, which supplies ever more diverse and detailed image data to a growing number of communities, is one of the reasons behind this increase. Furthermore, an understanding that DRM assessments have to go beyond capturing the physical impact, calls for a need for indirect measurements, which also contributed to this increase. 


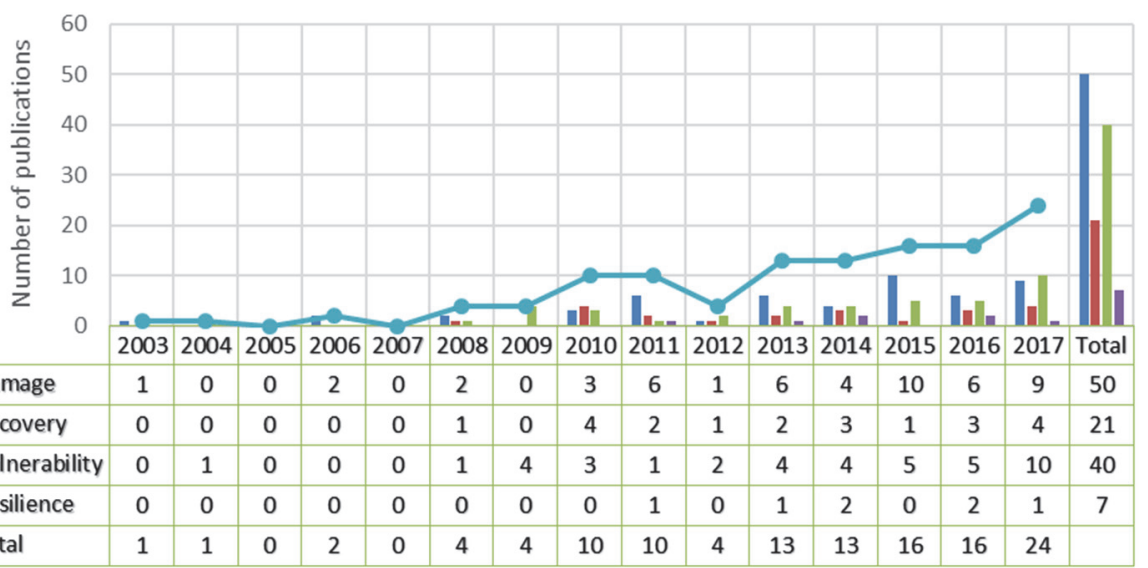

Figure 2.1 Number of annual publications on remote sensing-based proxies for Disaster Risk Management (DRM). Papers with a focus on two DRM areas, e.g., both damage and recovery assessments, are counted on both of them separately.

Figure 2.2 shows the number of RS-based proxies that have been developed for each component of DRM. The built-up environment is the most frequently studied one with 35 RS-based proxies developed. Natural environment accounts for the lowest number of developed RS-based proxies, offering a choice of 13. Furthermore, the Economic and Social environments both have 21 developed RS-based proxies. Some proxies that are used interchangeably in more than one area, e.g., damage and recovery assessments, are counted on all of the used categories separately.

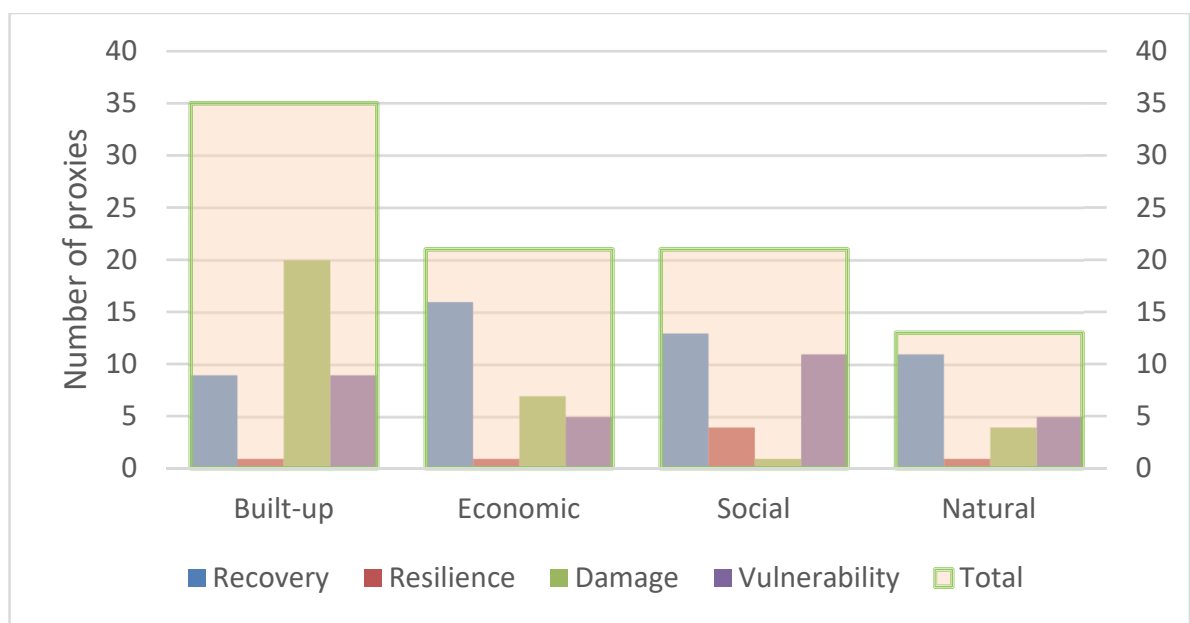

Figure 2.2 Number of developed remote sensing-based proxies for DRM in each environment (Built-up, Economic, Social, Natural). The proxies that are used interchangeably in more than one area, e.g., damage and recovery assessments, are counted on all of the used categories separately. However, the green colored bar shows the total number of unique RS-based proxies for each environment. 


\subsubsection{Built-Up RS-Based Proxies}

The build-up environment in urban areas tends to be significantly impacted during a disaster and these adverse impacts are relatively easy to detect. It makes this category the most studied in the RS literature (Figure 2.1), with the majority of RS-based proxies being developed here as compared to other environments within urban DRM (Tables 2.1-2.3). To discuss and explain the proxies in detail the built-up component is separated into three main categories: Buildings, Transport, Others.

\subsubsection{Buildings category}

The predominant land cover in urban areas is the buildings class. This is why one of the mostly used proxies for damage (Kerle and Hoffman 2013) and recovery (Brown et al. 2010b) assessments in urban DRM is extracting the status of the buildings. For example collapsed/damaged or reconstructed buildings are detected either using multi-temporal RS data by comparing the pre-event and post-event situations (Janalipour and Taleai 2016), or only using mono-temporal RS data acquired after the disaster (Turker and Sumer 2008). For example, Bevington et al. (2010) identify the damage level in urban areas after the Haiti earthquake by extracting buildings damage using qualitative analysis from satellite imagery along with the integration of visual field data. Similarly, Chen et al. (2003) use aerial photography to identify collapsed and damaged buildings (residential houses), and develop a damage pattern mining framework to detect them. Besides, the physical changes in the recovery processes are assessed mostly using a pathway from rubble of collapsed buildings to standing (reconstructed) buildings. Hence, Platt et al. (2016) use the reconstruction of buildings as a proxy for recovery assessment after an earthquake. Several studies also employ this proxy for physical recovery assessments (Brown et al. 2015; Brown et al. 2010a; Brown et al. 2011; Brown et al. 2008; Costa Viera and Kerle 2014; Platt et al. 2016). A reconstruction of some specific buildings (e.g. commercial buildings) is also used as a proxy for socio-economic recovery assessments (Costa Viera and Kerle 2014). However, all of them disregard the functional recovery assessment of buildings, which indicates whether a building is operating and/or the building is being used for its intended type of activity (e.g., commercial).

In addition, the value of point height change has been used as a proxy to extract information for grading the damage for each building using pre- and post-earthquake DEMs (Feng et al. 2013). Although this proxy provides a good source of information to assess per building damages, it cannot detect damages that do not necessarily change the value of the height of the buildings, such as cracks and holes in facades. 
Some proxies are considered as higher level ones due to their indirect observability in addition to indirect interpretations to indicate something meaningful for computation. One of them is shadow, which has been used in the DRM context to extract information about collapsed/damaged/reconstructed structures from RS images (Huang and Zhang 2012; Prince et al. 2017; Tian et al. 2017; Turker and Sumer 2008). Kerle and Hoffman (2013) provide a comprehensive discussion of pros and cons of using shadow as a proxy to detect building-based changes. They demonstrate that although missing or smaller shadows compared to pre-event situation may indicate a damaged or collapsed buildings (e.g. pancake collapse), the sun direction differences between the acquired images resulting in shadow changes, can lead to confusion. Image texture has frequently been used as a proxy to identify damaged/collapsed buildings (Dong and Shan 2013; Miura et al. 2013; Samadzadegan and Rastiveisi 2008; Tu et al. 2016), damaged roads (Sghaier and Lepage 2015; Ye et al. 2014) and, damaged areas (Cooner et al. 2016; Gao et al. 2015) from RS imagery. Damaged buildings/areas have more irregular texture than intact ones (Samadzadegan and Rastiveisi 2008); therefore, the damaged/collapsed ones can be extracted by comparing their textures. However, this proxy may not be reliable in detecting damages to informal settlement (slums) since they normally have irregular textures. Moreover, texture has been shown as an useful additional information for urban structure discriminations, and has been used for building seismic vulnerability analysis (Geiß et al. 2015; Geiß et al. 2016).

In addition, building roof offsets between adjacent buildings have been used as a proxy to detect pancake collapse, in which the building is characterized by an intact roof but collapsed floors (Kerle and Hoffman 2013). Building material has been used for vulnerability (Costanzo et al. 2016; Geiß et al. 2015) and resilience assessments (Qasim et al. 2016; Rezaie and Panahi 2015; Yuan Zx and Lm 2004). Building material detection from RS data is typically based on the interpretation of rooftop colors from aerial or satellite images without considering other materials of building (e.g. building wall materials). Data by drones/UAV on facades status have a potential to offer more reliable proxies for building material analysis/assessment due to providing information about the walls and other elements of the buildings in the facades in addition to their rooftops (Ilehag et al. 2017). Energy loss by buildings has been used as a proxy for recovery (Costa Viera and Kerle 2014), where a decrease in energy loss of a structure is considered as a sign of positive recovery as it indicates an improved building standard. This proxy is extracted based on the heat level of structures using hyperspectral imagery. A lower heat level of a house shows a lower energy loss level. The rate of energy loss reduction of houses demonstrates their insulation level and is related to building materials and overall construction quality. However, a non-functioning building does not produce energy (heat), and thus, when compared to others shows a low energy 
loss, which can lead to inaccurate results in the extraction of the insulation level of buildings. Meanwhile, this point also demonstrates that the energy loss of buildings can be used as a proxy for functional assessment of buildings. 


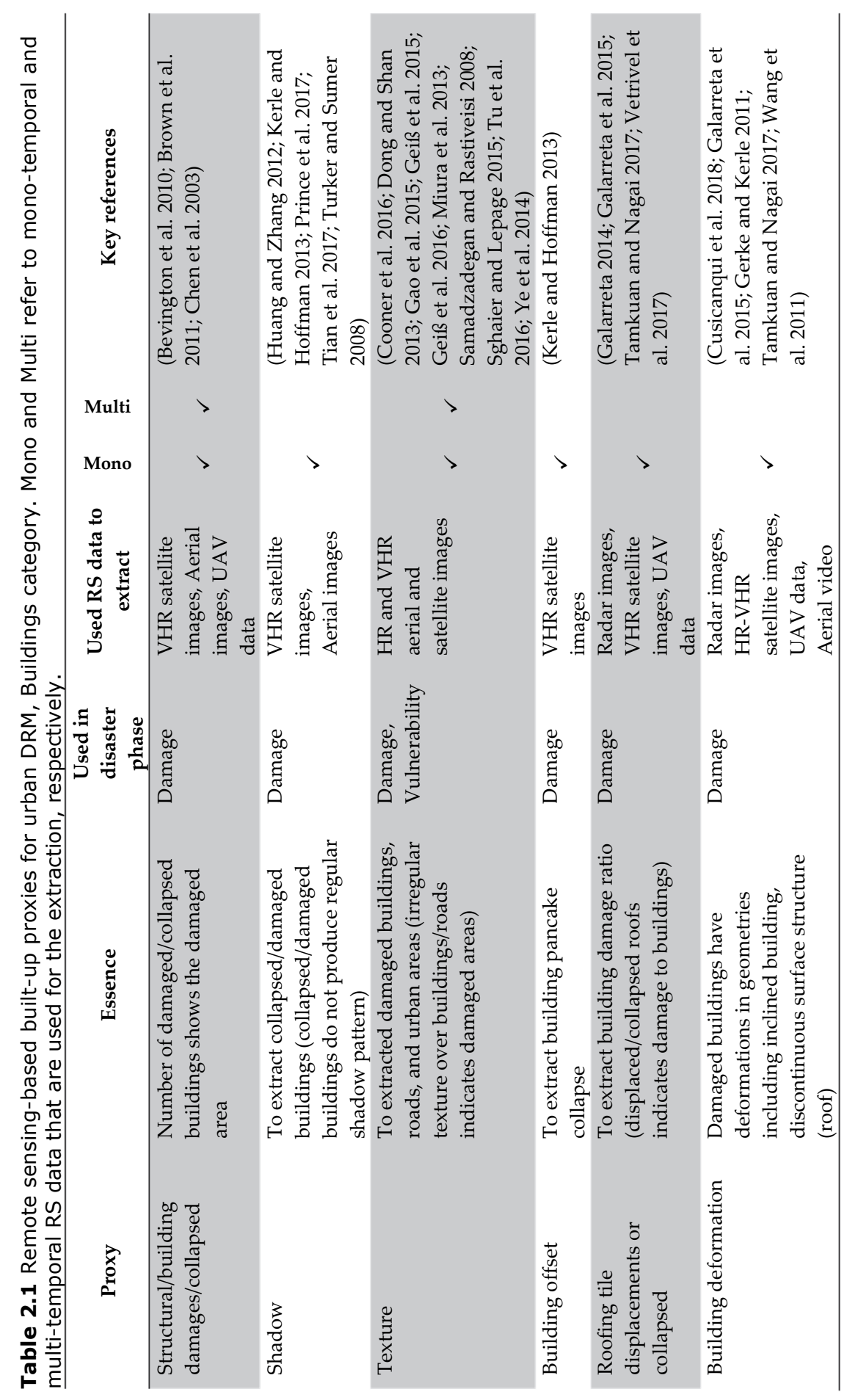




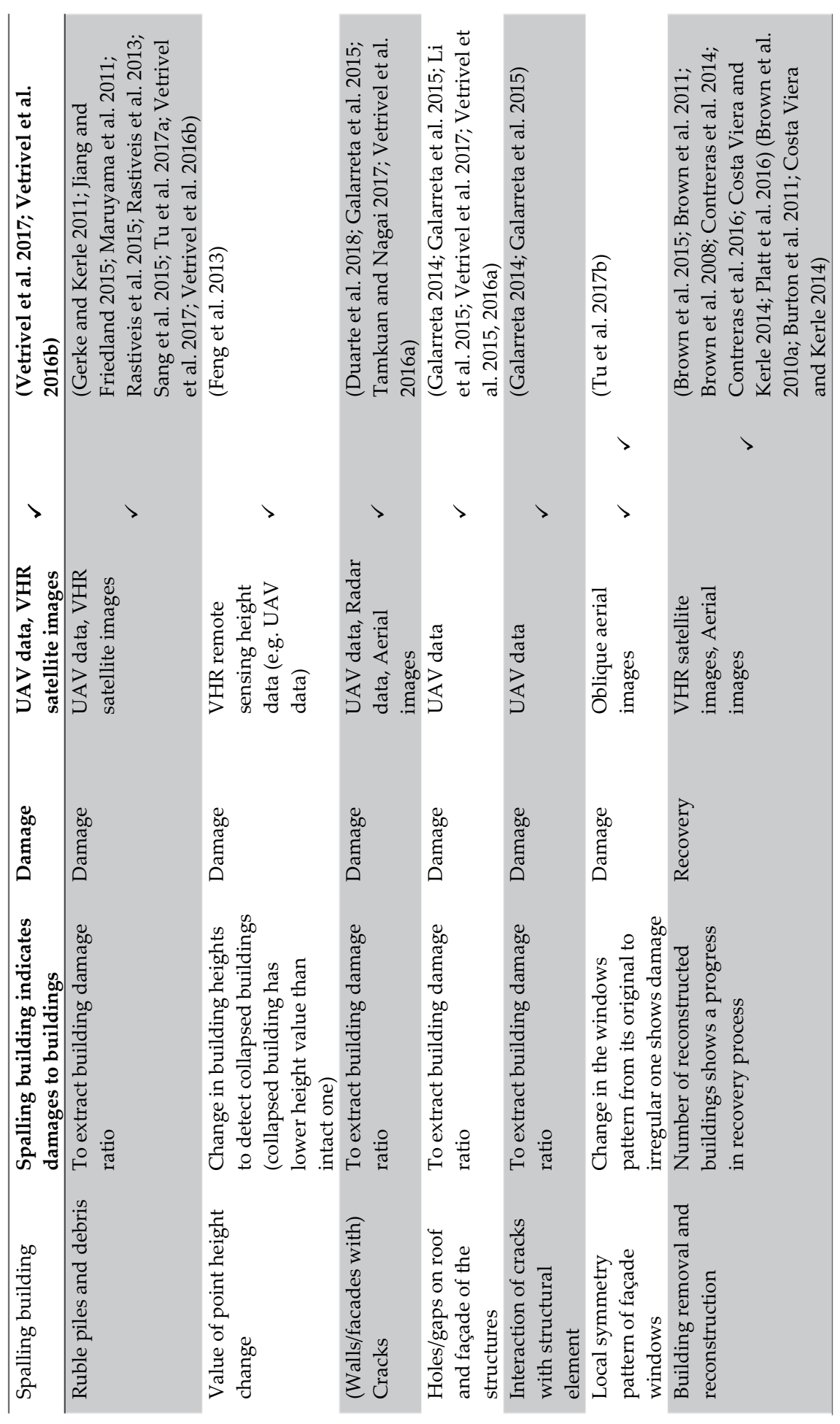




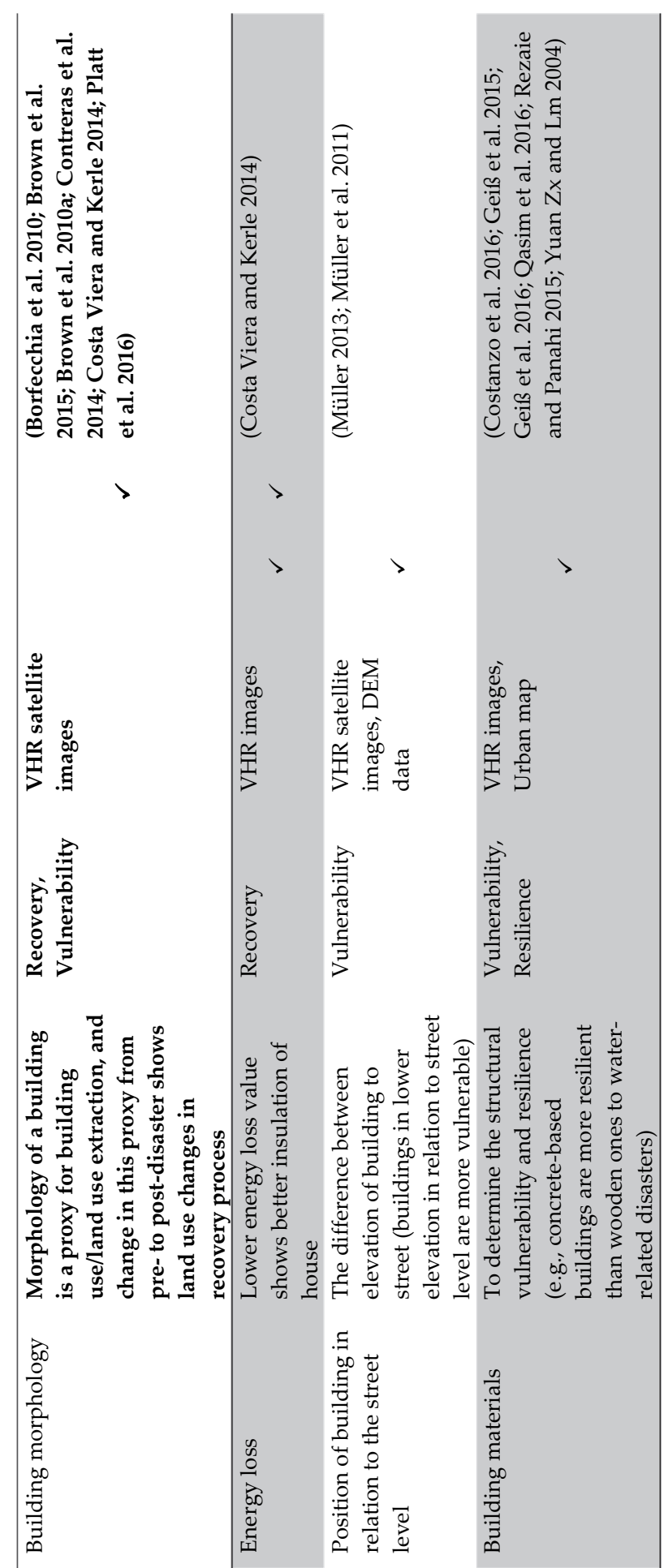




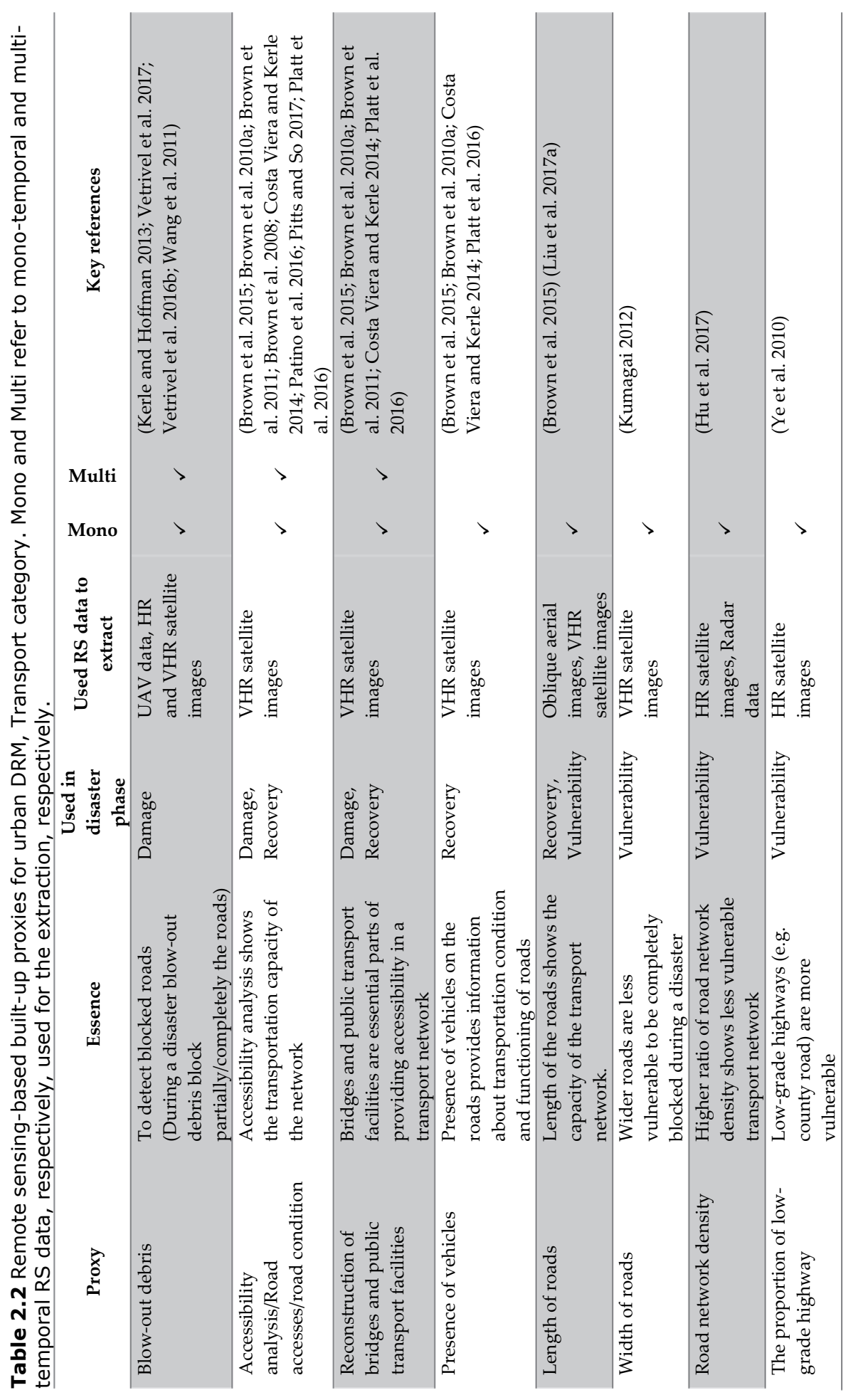




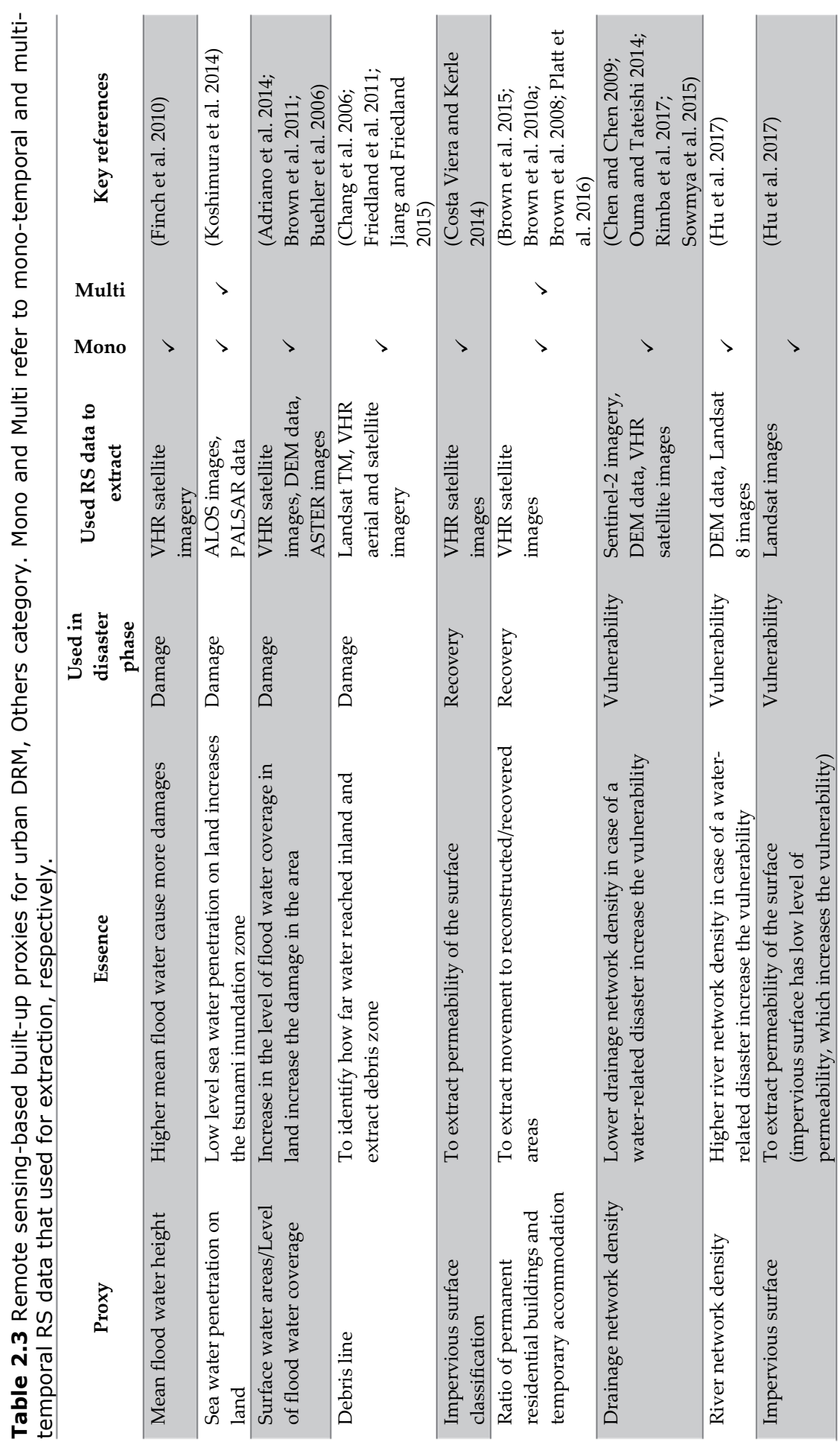



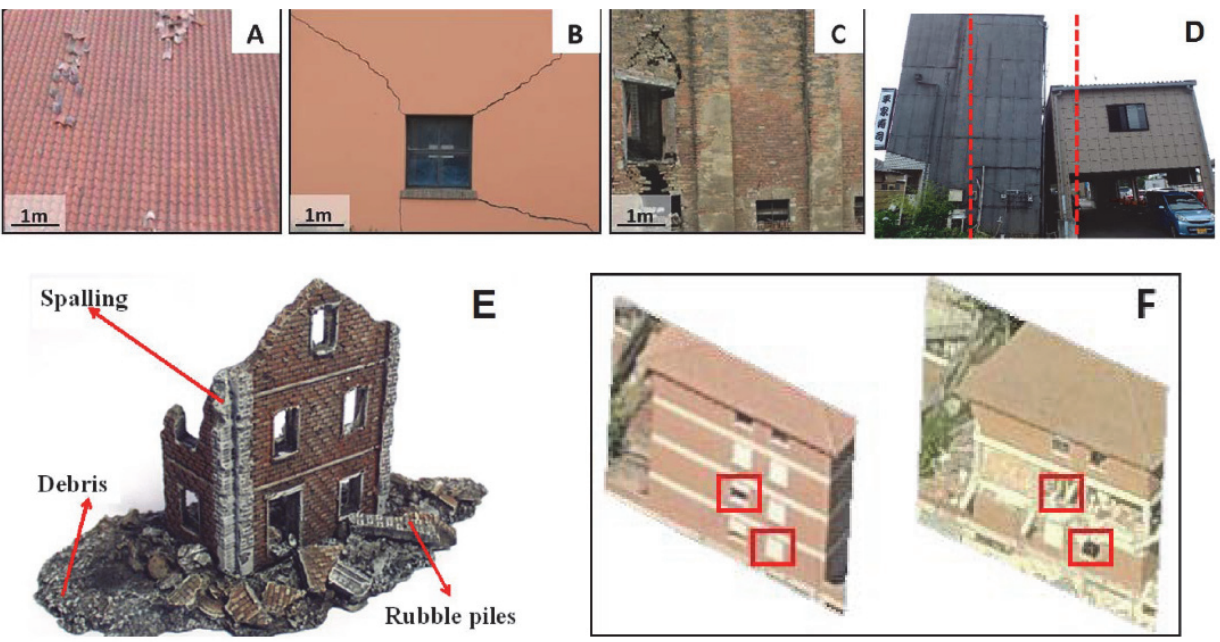

Figure 2.3 Examples of remote sensing-based built-up proxies. (a) Roof with dislocated tiles, (b) cracks in concrete façade, (c) cracks and hole in brick façade (Galarreta et al. 2015), (d) inclined building (Galarreta et al. 2015; Tamkuan and Nagai 2017), (e) debris, rubble piles, spalling (Vetrivel et al. 2016a) and (f) façade windows symmetry (Tu et al. 2017b).

Several proxies rely on geometric and morphological characteristics of built-up components to extract detailed information about structural deformations of buildings. They are used to compute the damage ratio of buildings. Gerke and Kerle (2011) use the discontinuity of surface of building rooftops and vertical walls as a proxy for damage detection. They show that continuous roofs indicate intact buildings. In addition, Galarreta et al. (2015) identify cracks and holes in the building roof or facades, intersections of cracks with load-carrying elements and dislocated tiles (Figure 2.3a-c) as proxies to be utilized to assess per-building damages after a disaster. Since these details can only be extracted using VHR images and height data, UAV images were used to produce 3D point clouds to extract them. The results illustrate the efficiency of using those proxies for building-based damage assessment and classification when UAV data are available. Tamkuan and Nagai (2017) used similar proxies: cracks, displaced and collapsed roofing tiles, wall mortar that is somewhat peeled off, or inclined buildings (Figure 2.3d) to evaluate and classify the building-based damages, e.g. roof tile displacements and inclined buildings are used as proxies for detecting slightly and heavily damaged buildings, respectively. Rubble piles and debris (Figure 2.3e) are the first features to draw attention and have been frequently used as proxies for damage detection (Jiang and Friedland 2015; Maruyama et al. 2011; Rastiveis et al. 2015; Rastiveis et al. 2013; Sang et al. 2015; Tu et al. 2017a), while spalling of buildings (Figure 2.3e) also is one of the features for heavy damages which has been used for damage detection with UAV (Vetrivel et al. 2017; Vetrivel et al. 2016a) and satellite images (Vetrivel et al. 2016b). Furthermore, geometric deformation of entire buildings, 
such as changes in building shape and size, is employed as a proxy to extract the partly damaged buildings using satellite images (Wang et al. 2011). Change in the symmetry of windows on the façade of a building serves as a proxy for damage assessment from oblique aerial imagery (Tu et al. 2017b). To do so, local symmetry points are detected in a sliding window. Then histogram bins of those points in the vertical and horizontal directions are generated to distinguish damaged and undamaged building facades using the Gini Index. Using changes to different components of the buildings, e.g. façade and wall, as a proxy to evaluate damages to the buildings in detail remains a challenge and continues to be actively studied (e.g. Duarte et al. (2018)).

Further improvements are required for robust and accurate damage assessment per building. This can be done by extracting structural damage patterns from RS data, for example by making use of the characteristics of façade wall geometry, similar to using façade windows symmetry (Figure 2.3f) (Tu et al. 2017b), wall slenderness, area of wall, or use of misalignment of wall openings (Ferreira et al. 2014) as RS-based proxies.

Building morphology acts as a proxy to extract building-based land use (Borfecchia et al. 2010; Brown et al. 2015; Contreras et al. 2014; Platt et al. 2016) and to measure living conditions (Brown et al. 2010a; Brown et al. 2010b; Costa Viera and Kerle 2014). It has been used for post-disaster recovery and vulnerability (Borfecchia et al. 2010) evaluations, but hardly in other DRM situations. In a different approach, Müller et al. (2011) apply the position of buildings in relation to the street level as a proxy to determine the likelihood of constructions to suffer damage in case of a flood event and assess the building vulnerability. Accordingly, buildings with lower elevations than the street are more vulnerable.

Building-based functional assessment has not been sufficiently addressed through the developed proxies that only consider the land use information. For example, functionality of the buildings may change during the post-disaster recovery phase, which cannot be identified only by extracting the reconstructed buildings. Nevertheless, façades are good sources of information for buildingbased functional assessment, and new proxies could make better use for example of the presence of cars in a driveway, flower pots in front of windows of residential buildings, or presence of panel and banners on top of shops for extracting the functional use of buildings.

\subsubsection{Transport category}

Transportation is also a vital component for DRM as it provides accessibility to different locations within urban areas. One of the first emergency activities in post-disaster situations is to reopen blocked roads to reach damaged areas for rescue operations. Road network connectivity and condition, i.e. accessibility 
(Pitts and So 2017) has been used as a proxy for damage assessment. In addition, monitoring this proxy over a long period of time after a disaster also contributes to measuring recovery of the area (Brown et al. 2010a; Costa Viera and Kerle 2014). In terms of more detailed proxies, debris on the road (blocked road) (Brown et al. 2011) and condition of bridges and transport facility (Platt et al. 2016) have been proposed to evaluate the transportation condition for urban DRM. Presence of vehicles has also been used as a proxy to evaluate the accessibility of the roads after a disaster (Brown et al. 2010a). However, the presence of vehicles on a road does not necessarily signal good condition and usability of the roads. On the other hand, reconstruction of bridges and roads is a reliable proxy for road accessibility analysis after a disaster.

In another study, Liu et al. (2017a) utilize length of roads as a proxy to evaluate the storm surge vulnerability of urban area, and Brown et al. (2015) use it for recovery assessment. However, a non-functioning road does not demonstrate the successful recovery processes. In a different study, Kumagai (2012) estimate width of roads as a proxy to assess the vulnerability of the roads to blockades. The results show that in a dense urban area, in which highrise buildings exist and their blow-out debris or in case of collapsing during a disaster event can block the road, width of roads can be used as a reliable proxy to assess the vulnerability of the transport network. In a similar study, Hu et al. (2017) rely on road network density as a proxy for disaster vulnerability assessment. Although denser road networks and wider roads can increase the chance of having access to damaged areas after a disaster, occupying urban areas to reach denser road ratios and wider roads is a controversial. Similar problems exist in using proportion of low-grade highways such as county roads (Ye et al. 2010) as a proxy for vulnerability assessment of a city. High proportion of low-grade highways indicates a less developed transportation infrastructure, which leads to higher level of vulnerability of urban areas.

\subsubsection{Others}

One of the unique features of tsunami inundation zones important during and after an event is the level of sea water penetration on land. Thus, RS studies have focused on extracting water bodies from the post-event images as the first step to use it as a proxy for damage assessment (Koshimura et al. 2014). Similarly, surface water areas (level of flood water coverage) serves for damage (Adriano et al. 2014; Buehler et al. 2006) and recovery assessments (Brown et al. 2008). Furthermore, debris line acts as a proxy to identify the impact of water related disasters such as tsunami (Chang et al. 2006) and hurricane (Friedland et al. 2011). The debris line demonstrates how far water reached inland after a disaster, and it has also been used to identify debris zone (Jiang and Friedland 2015). 
In a different study, impervious surface ratio is estimated as a proxy to compute vulnerability of urban areas because the increase of impervious surface can lead to an increase of volume, intensity, and duration of urban water run-off, i.e. the duration that water remains on the surface after a flood (Hu et al. 2017). In addition, Costa Viera and Kerle (2014) used impervious surface as a measure for recovery assessment.

As a unique recovery situation, after a disaster people that are located in a temporary shelter during the reconstruction processes move back to their permanent houses when the reconstructions is completed. Consequently, change in the ratio of permanent buildings to temporary accommodations serves as a proxy for movement of the population from temporary accommodation to permanent houses and as a successful recovery process (Brown et al. 2015; Brown et al. 2008; Platt et al. 2016).

After a flood event, drainage plays an important role to evacuate flood waters, with an increase in drainage density reducing the vulnerability from the affected area; therefore, it has been used as a proxy for urban flood vulnerability assessment (Chen and Chen 2009; Ouma and Tateishi 2014; Rimba et al. 2017; Sowmya et al. 2015). In contrast, a higher river network density ratio indicates a high vulnerability due to the relatively larger amount of flood-prone regions during a flood event (Hu et al. 2017).

\subsubsection{Economic RS-based proxies}

The nature of economic and business flows associated with functions and processes in urban areas is not directly observable and measurable through RS data. Hence, the disruptions to the functioning of the economic environment is one of the least studied topic in urban DRM. To discuss and explain the proxies for the economic component in details, we differentiate between two main categories - (macro, regional and urban) economics and logistics - that characterize the state of an economy and assure physical flows of economic activities correspondingly.

\subsubsection{Macro, regional and urban economics}

The macro, regional and urban economics category includes proxies that are associated with the performance of the economy as a whole and its associated changes (Table 2.4). Land use data provides information about locations and types of economic activities. In the urban context it is one of the most popular proxies used to identify disaster-related economic damage for different economic sectors (Brown et al. 2011), as well as recovery (Costa Viera and Kerle 2014) and vulnerability (Guntur et al. 2017). For example, Brown et al. (2011) applied the building-based land use proxy to identify damages to commercial and industrial buildings when assessing economic damages from 
the Wenchuan earthquake in China. Similarly, it has been also used for recovery assessment by extracting reconstructed buildings associated with economic activities (Brown et al. 2010a; Costa Viera and Kerle 2014). The land use proxy and corresponding economic activities, their location, public infrastructure, economic assets and economic capacity, has also been used for economic vulnerability evaluations of urban areas (Liu and Shi 2017). Although land use is a crucial source of information with respect to direct impact on economic activities, it is salient on the detailed economic information such as type of businesses and operationally of the impacted buildings. Moreover, while land use proxies can capture direct economic damages to physical assets, it provides little information about direct damage to building content (e.g. equipment and furniture) and about indirect damage due to business interruption.

Nightlight satellite imagery has been utilized to assess the economic impacts of hurricanes (Bertinelli and Strobl 2013), typhoons (Elliott et al. 2015), and tropical cyclone (Mohan and Strobl 2017). Nightlight images give an opportunity to compare changes in night light intensity, which relates to electricity availability, for before and after a disaster. It has been shown that a strong correlation exists between night light intensity and GDP (Mellander et al. 2015; Proville et al. 2017). Furthermore, it has also been suggested as a potential proxy for economic resilience assessment (The World Bank 2014). 


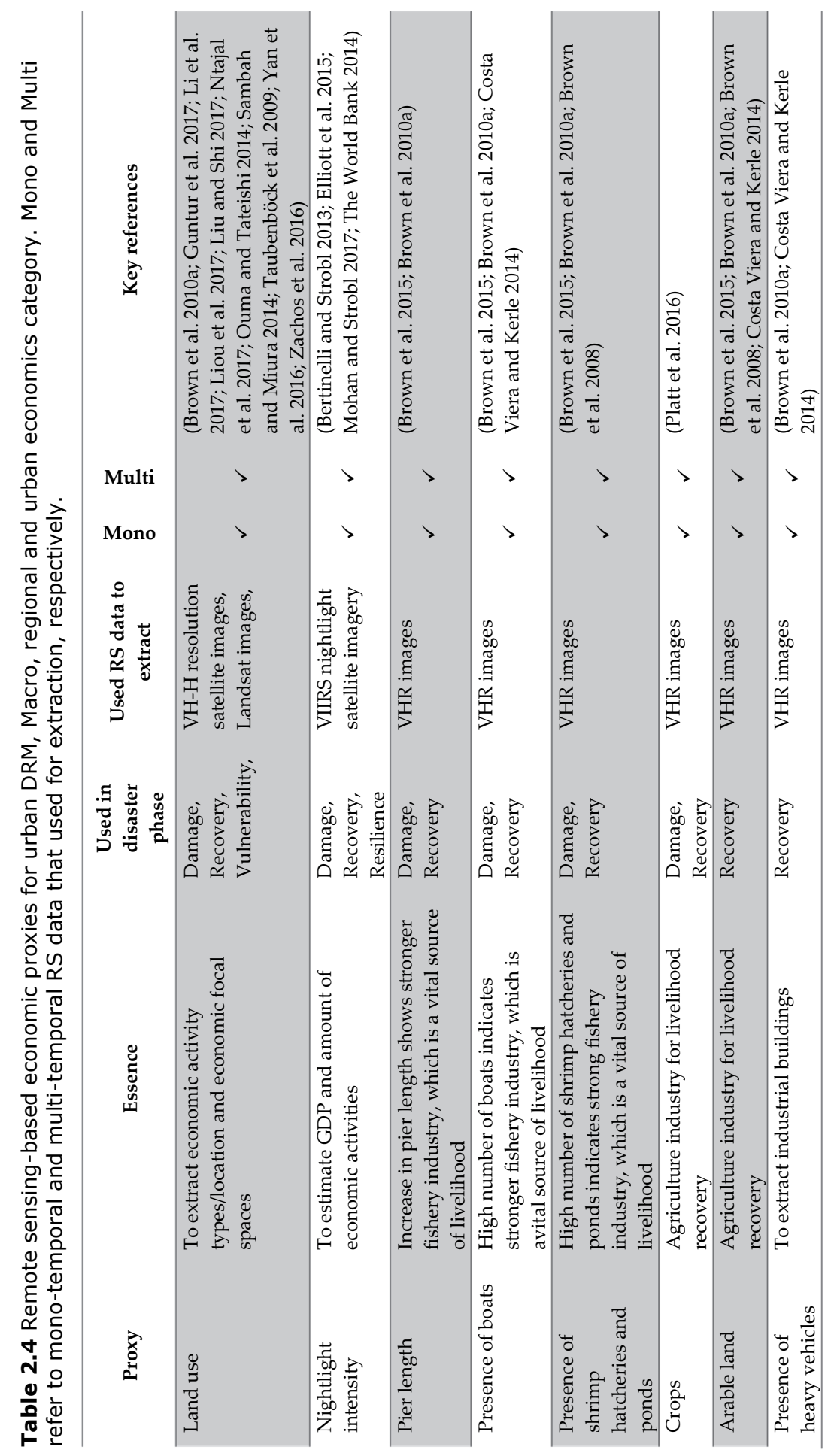




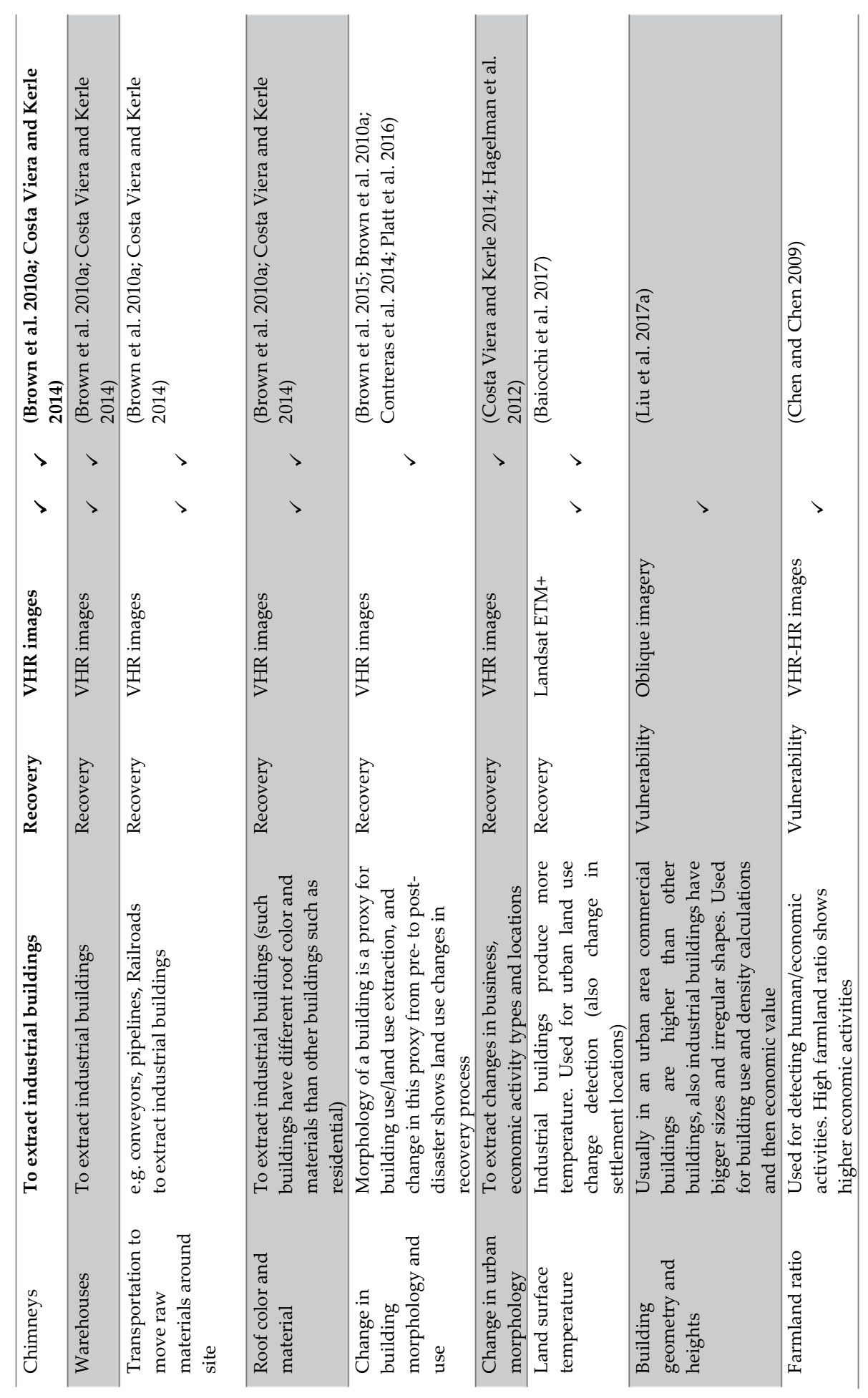


Disruption of livelihood options for local population is a key component of disaster risk and resilience assessments. A number of case-based proxies assess a short-term economic recovery processes after a disaster, in particular for the fishery and agricultural livelihood activities. These proxies include pier length, presence of boats (Brown et al. 2010a), shrimp hatcheries and ponds (Brown et al. 2008), and area of arable land (Costa Viera and Kerle 2014). Although livelihood sources across study regions, generic proxies can address it in a more reliable manner than case-based ones, for example as with the case of crop recovery (Platt et al. 2016).

Industry is one of the main pillars of an economy. Therefore, urban proxies to assess industrial recovery include presence of heavy vehicles, chimneys, warehouses, transportation to move raw materials around site (e.g. conveyors, pipelines, railroads), and roof color and materials (Brown et al. 2010a; Costa Viera and Kerle 2014). These proxies for detecting industrial buildings and industry-related activities are especially useful for assessing recovery associated with rebuilding and reconstruction. However, they are not always reliable when used in isolation. For example, the effectiveness of using the presence of heavy vehicles to detect an industry highly depends on the acquisition time of RS data, not to mention that not all industries use heavy vehicles. Hence, using multiple complementary proxies promises to deliver more robust assessments. Furthermore, building façade materials, in addition to rooftop materials, can also contribute to extract building use/land use information (Ilehag et al. 2017).

Building morphology is used as a proxy for extracting building use/land use information in urban areas (Brown et al. 2010a; Platt et al. 2016). Characteristics of buildings such as size and shape have been used to extract morphological information per building and then to classify building use (Contreras et al. 2014). For example, an industrial building usually has a complex shape and a large size (Li et al. 2016a). Similarly, and from a wider point of view, change in urban morphology has been used for economic recovery assessment by detecting businesses movements and types in large scales (Hagelman et al. 2012).

Land surface temperature was used to extract evolution of settlement locations and land use information after the earthquake in L'Aquila city, Italy (Baiocchi et al. 2017). This proxy is robust when used on coarse resolution data to extract large size settlement changes. However, it cannot identify any detailed land use information for those settlements.

There are several RS-based proxies developed for economic vulnerability assessment, in contrast to other DRM components. For instance, building geometry and heights have been employed as proxies to assess the number of 
floors of buildings with economic activities. These two proxies may indicate the density of such activities, which is essential for computing the economic value of an urban area (Liu et al. 2017a). Building heights, when integrated with local construction regulations, provide reliable data to compute the number of floors. Moreover, a calculation of building density based on the number of floors is reliable (Yu et al. 2010). However, it requires additional RS data such as oblique imagery or nDSM data (Aubrecht et al. 2009).

Farmland ratio to other types of land uses is strongly linked to economic activities and can be regarded as a comprehensive characteristics of socioeconomic systems (Chen and Chen 2009). Accordingly, it has been used as a proxy to detect economic activity, with higher scores signaling lower economic vulnerability in e.g. a flood scenario [refs]. However, in large regions, which offer multiple livelihood options, the farmland ratio alone is not a reliable proxy for identification of economic vulnerability. Moreover, a level of diversification of economic activities is an important factor in assessing the resilience (Cutter 2016; Rose and Krausmann 2013). Hence, eliciting spatial proxies for a variety of economic in an ensemble is a promising approach compared to computing farmland ratio alone.

\subsubsection{Logistics}

Logistics based on transport networks and facilities are vital aspects of a strong economy in urban areas. Consequently, their state and performance are important for urban DRM economic assessment (Table 2.5). Gil and Steinbach (2008) compute the impact of a simulated disaster on the performance of the street networks that are not directly affected by a flooding scenario in London. The potential of street network analysis is to provide objective indicators of indirect impacts of flooding on urban street networks in unaffected areas. The indirect effects of business interruption, particularly in a city center, play a key role in assessing an economic performance after an adverse event. In a similar study, Contreras et al. (2013) propose spatial connectivity as a proxy for a post-earthquake recovery assessment. Variables such as distance, travel time and quality of public transportation are used to compute this metric. These authors conclude that an efficient spatial connectivity of areas to the central business district of L'Aquila after an earthquake can decrease the recovery time due to an agile return of economic functioning. Cox et al. (2011) indicate that an accessibility and commuting intensity within an urban area - computed based on resources availability, vulnerability, and flexibility of the transportation system - significantly boost economic activities. Accordingly, Hsieh and Feng (2014) propose a model for accessibility analysis as a proxy to compute an economic vulnerability of an urban are. This proxy is mostly based on streets connectivity in an urban road network. However, the proposed connectivity-based approaches need traffic data in addition to RS data to compute street network performance. In a different study Liu and Shi (2017) 
show that a higher ratio of transportation lands (e.g. roads) and facilities reduces economic vulnerability of urban areas. However, this proxy also disregards the functional analysis of the roads and transportation facilities focusing on the physical infrastructure side only.

Table 2.5 Remote sensing-based economic proxies for urban DRM, Logistics category. Mono and Multi refer to mono-temporal and multi-temporal RS data that used for extraction, respectively.

\begin{tabular}{|c|c|c|c|c|c|c|}
\hline Proxy & Essence & $\begin{array}{l}\text { Used in } \\
\text { disaster } \\
\text { phase }\end{array}$ & $\begin{array}{l}\text { Used RS } \\
\text { data to } \\
\text { extract }\end{array}$ & 3. & 竝 & $\begin{array}{c}\text { Key } \\
\text { references }\end{array}$ \\
\hline $\begin{array}{l}\text { Indirect street } \\
\text { network } \\
\text { performance }\end{array}$ & $\begin{array}{l}\text { Impacts of an event } \\
\text { not only on the } \\
\text { effected roads but } \\
\text { also on the other } \\
\text { streets }\end{array}$ & Damage & $\begin{array}{l}\text { VHR } \\
\text { images }\end{array}$ & $\checkmark$ & $\checkmark$ & $\begin{array}{l}\text { (Gil and } \\
\text { Steinbach } \\
2008 ; \\
\text { Penchev } \\
\text { 2016) }\end{array}$ \\
\hline $\begin{array}{l}\text { Spatial } \\
\text { connectivity }\end{array}$ & $\begin{array}{l}\text { Spatial connectivity } \\
\text { to central business } \\
\text { district }\end{array}$ & Recovery & $\begin{array}{l}\text { VHR } \\
\text { images }\end{array}$ & $\checkmark$ & $\checkmark$ & $\begin{array}{l}\text { (Contreras et } \\
\text { al. 2013) }\end{array}$ \\
\hline $\begin{array}{l}\text { Transportation } \\
\text { land and } \\
\text { facilities }\end{array}$ & $\begin{array}{l}\text { High level of } \\
\text { transportation } \\
\text { facilities reduces } \\
\text { economic } \\
\text { vulnerability }\end{array}$ & Vulnerability & $\begin{array}{l}\text { VHR } \\
\text { images }\end{array}$ & $\checkmark$ & & $\begin{array}{l}\text { (Liu and Shi } \\
\text { 2017) }\end{array}$ \\
\hline $\begin{array}{l}\text { Accessible } \\
\text { vulnerability/ } \\
\text { connectivity }\end{array}$ & $\begin{array}{l}\text { Fragile accessibility } \\
\text { increases the } \\
\text { economic } \\
\text { vulnerability }\end{array}$ & Vulnerability & $\begin{array}{l}\text { VHR } \\
\text { images }\end{array}$ & $\checkmark$ & & $\begin{array}{l}\text { (Hsieh and } \\
\text { Feng 2014) }\end{array}$ \\
\hline
\end{tabular}

\subsubsection{Social RS-based proxies}

The social dimension dealing with the status of people in a community is one of the most important components in DRM. Yet, few reliable RS-based proxies exist to address it. This is mostly because the social dimension of disasters embraces an individual status of community members, diversity of livelihoods (economic activities they may engage in), level of inequality, community cohesion and level of services. Hardly any of these are directly captured through physical infrastructure or other spatial data that can be extracted using RS data. Tables 2.6 and 2.7 show existing RS-based proxies in the social environment for urban DRM. To discuss and explain the proxies in detail we separate them into two categories: services and infrastructures, and socioeconomic status.

\subsubsection{Services and infrastructures}

Social services and infrastructures are one of the most important tools to assess the social features and characteristics of a community in an urban area. Accordingly, several proxies relying on RS data can be used in the urban DRM context. Urban primary social facilities and services, such as administrative 
services, schools, healthcare facilities and religious buildings, which are crucial sources for networking and building social ties and cohesion, provide a proxy information on social damage and recovery assessments (Brown et al. 2015; Brown et al. 2010a; Platt et al. 2016). In addition, local facilities in use such as car parking, highways, city gardens, children playgrounds, sport playgrounds have been used to assess the social condition of the people living in the shelters and temporary accommodation in recovery processes (Brown et al. 2010a; Contreras et al. 2017; Costa Viera and Kerle 2014). Meanwhile, the number of urban facilities such as hospitals and schools serve as a proxy to assess the social resilience of a community to disaster. An increase in the number of facilities indicates an increase in social resilience (Contreras et al. 2017).

To prevent a disease outbreak during the disaster recovery stage it is crucial to monitor and avoid overcrowding in campus/temporary settlement sites (Jafari et al. 2011). Here the minimum covered living space can contribute to measuring overcrowding in the site using RS (Brown et al. 2010a; Costa Viera and Kerle 2014).

During a recovery process, moving population that has been located in temporary accommodation back to their permanent houses is one of the positive processes because it increases people's ability to return to normal lives and offers stability. Therefore, temporary accommodation size -as a proxy to estimate the total population living in temporary accommodation- can shed light on the quality of life (Brown et al. 2010a; Costa Viera and Kerle 2014). However, in cases where the temporary accommodation is not removed, the movement of population cannot be monitored using this proxy. Moreover, the destination of the population movement cannot be extracted using this proxy. For example, people may migrate to other cities rather than moving back to their former houses, which happened on a large scale in New Orleans after hurricane Katrina (Sastry and Gregory 2014).

Pedestrian access/mobility serves as a proxy for evaluation of the recovery processes that is linked to urban transportation facilities (Song and Knaap 2004). It indicates inequalities in mobility and access of people to social facilities, which depends on the size of block parcels in the urban area, number of street network intersections and distance to those facilities (Costa Viera and Kerle 2014). Building height, similar to its use in the economy environment, indicates social recovery. For example, commercial and industrial developments indicate employment facilities and new job opportunities (Costa Viera and Kerle 2014). However, often buildings are in a mixed use, i.e. half commercial and half residential, limiting the application of this proxy. In this case, using UAV/oblique images, which contain information about the façades of the buildings, can be useful. For example, one can extract signs/banners of 
shops located on buildings' façades to identify a functional use of these buildings. Number of inhabitants per settlement indicates the recovery processes, serving as a proxy for a contentment level of new settlements. Low occupancy numbers in new developments demonstrate the dissatisfaction in new settlements, usually due to lack of facilities, infrastructure and job opportunities (Engineering 2013). RS data is used to detect new settlements and demographic data to extract number of inhabitants (Contreras et al. 2017). Transportation facilities are a vital infrastructure. Hence, their availability define the better life condition for the people living in the region and form the basis for assessing social vulnerability of an urban area (Liu et al. 2017a). In addition, a distance between buildings and lifelines (e.g., hospitals) is important in emergency cases: the longer this distance the higher is the social vulnerability of urban areas (Armenakis and Nirupama 2013; Ebert et al. 2009; Patino et al. 2016; Roy and Blaschke 2015).

Potential to evacuate based on the density of available roads (road $/ \mathrm{km}^{2}$ ) has been used as a proxy to assess social resilience of an urban area during floods (Kotzee and Reyers 2016). Although the availability of evacuation points is important, distance to them and early warning systems are also crucial. As another disaster resilience proxy, presence of open spaces including free and green areas and the street networks have been used for providing services, gathering and social interactions after/during a disaster (e.g. an earthquake (Villagra et al. 2014)). In addition, open spaces such as hills and street networks are significant for evacuation purposes (Allan and Bryant 2011; Villagra et al. 2014) 


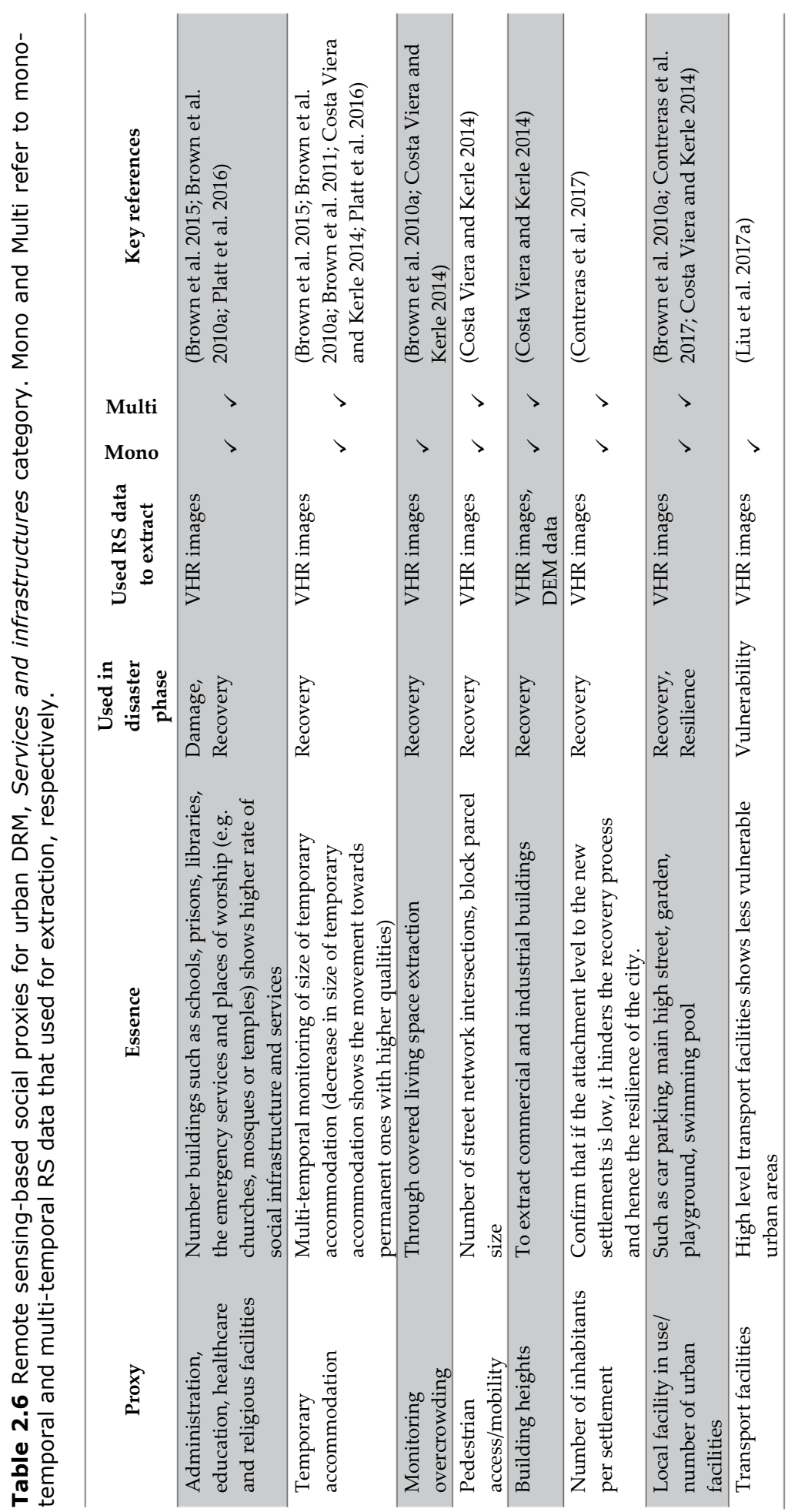




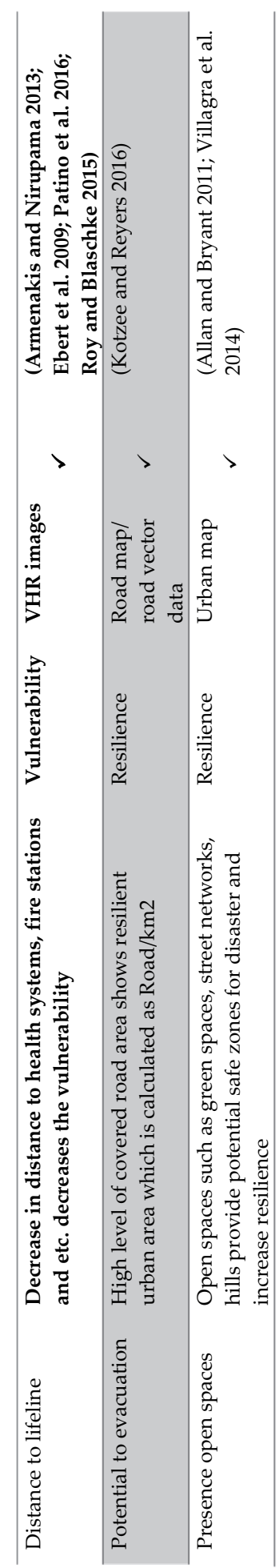




\subsubsection{Socio-economic status}

The socio-economic status of households is estimated based on the location information of the houses in the city and topographic area characteristics for social recovery and vulnerability assessments (Costa Viera and Kerle 2014; Ebert et al. 2009). Urban areas with poor road quality, little available infrastructure and economic development, little vegetation and situated in hazard-prone areas are the most socially vulnerable areas. However, these proxies are not necessarily correct for all cases in urban areas. For example, not all people located in hazard-prone areas (e.g. steep slopes or lower elevations) have a low socio-economic status; in contrast, in some cases there are luxury buildings located in areas with steeper slopes (Ebert et al. 2009; Stefanidis and Stathis 2013) or close to a waterfront (Huang and Li 2016; Xian et al. 2015). Therefore, an effective way of using those proxies is to use them as an ensemble to describe the socio-economic status of an urban area. In addition, image-based texture serves as a proxy to extract homogeneity of an area (Ebert et al. 2009; Mück et al. 2012; Sumaryono 2010). It is a good complementary source of information for a settlement type extraction from images, e.g. slum areas, even using medium resolution images (Kuffer et al. 2016). Similar to texture analysis, De Almeida et al. (2016) used irregular clusters of roofs in an urban area as a proxy to extract socially vulnerable areas, based on the fact that buildings poorer area are often not regularly constructed. A share of slum areas in a city compared to regular or high-income housing may serve as an indicator of social vulnerability. Müller (2013) also used the amount of green spaces around each building block to estimate the socio-economic status of householders based on the findings of Stow et al. (2007). Accordingly, the occupants of buildings surrounded by more green spaces - estimated using land cover and land use information to extract socioeconomic status - are less socially vulnerable to hazards. 


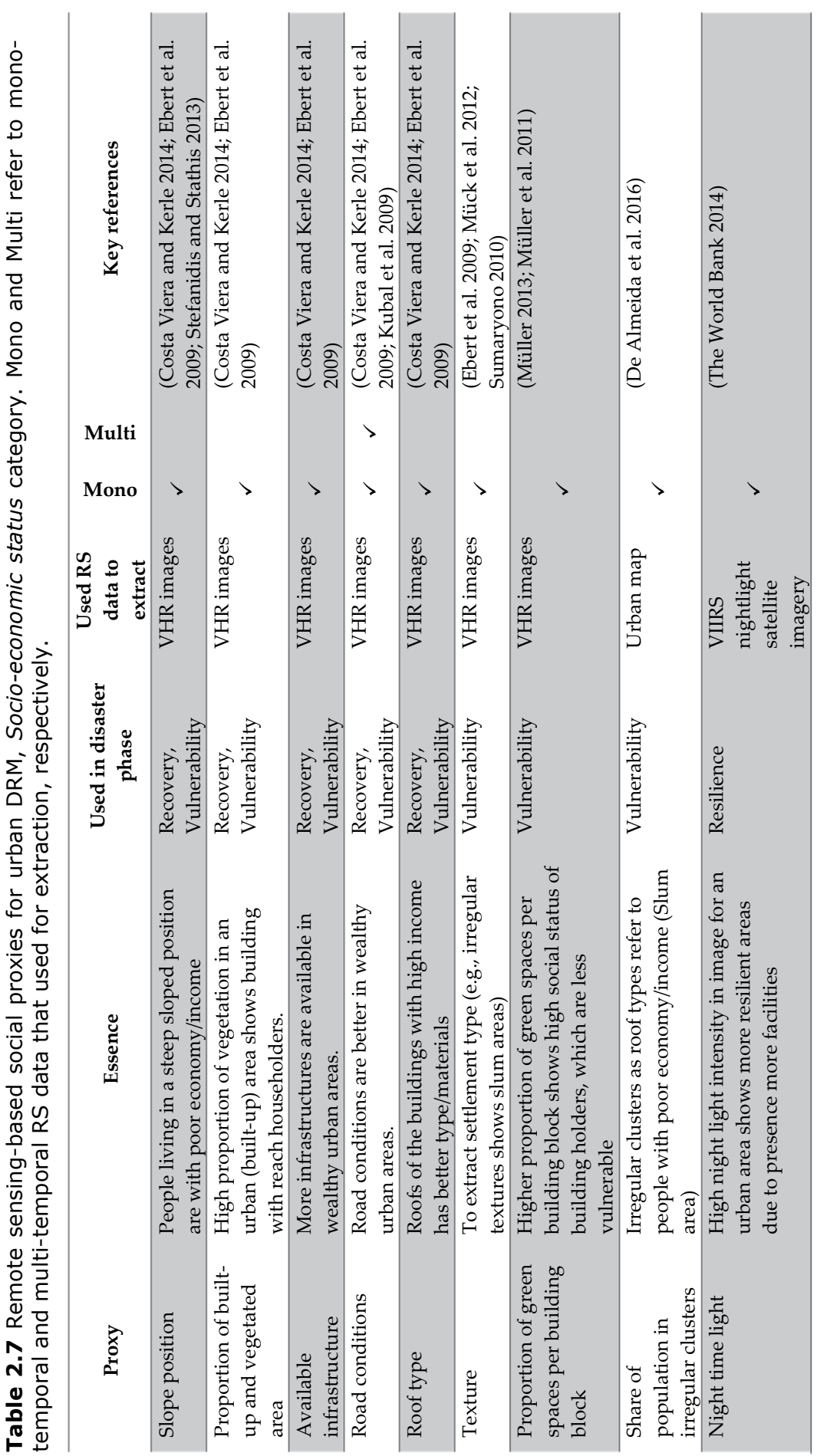




\subsubsection{Natural RS-based proxies}

Table 2.8 lists existing RS-based proxies capturing the natural environment for urban DRM. As one of the most important sources of information for assessing urban DRM, the natural environment relies on the vegetation cover information. Vegetation spatial heterogeneity serves as a proxy to extract changes in vegetation pattern, and to monitor ecosystem changes before and after a disaster at the landscape scale for natural damage assessment (Wang et al. 2017). Spatial heterogeneity may affect functions and processes in ecological systems, which is a key to assess ecosystem changes (O'neill et al. 1988). Vegetation cover has been used as a proxy to extract the damages to the natural and ecological environment of an urban area by comparing the vegetation cover before and immediately after a disaster (Adriano et al. 2014; Yang et al. 2011). Decrease in vegetation cover ratio of an area demonstrates the ecological damage ratio. In a different study, Li et al. (2016b) use vegetation cover change to assess the long-term effects of a hurricane in the urban natural environment recovery in New Orleans after Hurricane Katrina. Specifically, using MODIS and Landsat images, Li and colleagues assess the period from immediately after the event to ten years later to discover that a decade later the vegetation cover is still at a lower level than before Katrina, indicating that the area has not yet fully recovered. Similarly, Brown et al. (2011) and Brown et al. (2008) use vegetation cover maps to assess the changes in vegetation/natural environment patterns in the recovery processes using NDVI between year1-year2. In addition, the cover ratio for various vegetation classes can provide detailed and reliable information for damage and recovery assessments. Fractional vegetation cover (Jiao et al. 2014; Yang and Qi 2017), which represents the vegetation ecological function, and vegetation cover type (Yang and Qi 2017), have also been used to assess the vegetation recovery after a disaster. Fractional vegetation index can be computed using NDVI values of vegetation and soil, and is a powerful tool for vegetation cover classification and extraction (Liu et al. 2009). A change analysis allows to compute the vegetation recovery rate. In addition, vegetation offers an important ecosystem service in flood-prone areas by providing a low drainage density and high permeable surfaces, thus contributing to preventing floods (Pallard et al. 2009). Accordingly, vegetation cover ratio has also been used as a proxy for vulnerability assessment of urban areas, particularly for water-related hazards (Chen and Chen 2009; Liu et al. 2017a; Rimba et al. 2017).

Furthermore, vegetation cover ratio has been used as a proxy to evaluate the biophysical (Rimba et al. 2017) and ecological (Zhang et al. 2015) vulnerability, as well as resilience. A higher ratio of vegetation cover signified and increase in resilience (the vegetation coverage is one of the most important indicators for measuring ecological capacity for self-restoration). In 
a similar study, Mainali and Pricope (2017) use Standard Deviation of NDVI as a proxy to extract diversity of the plants in vegetated areas for vulnerability assessment. Debris and floodwater removal are also important for environmental recovery after a disaster, as it provides space for the vegetated area to regrow (Brown et al. 2008; Burton et al. 2011; Costa Viera and Kerle 2014; Finch et al. 2010; Platt et al. 2016).

The environmental role of public spaces and urban green areas, in addition to providing clean air, is to provide habitats for biodiversity and helps to regulate temperature. Therefore, it has been used as a proxy to assess natural recovery processes (Brown et al. 2015; Brown et al. 2010a).

Presence of debris, mud and salt in water as an indicator of water contamination (Brown et al. 2010a; Costa Viera and Kerle 2014) and availability of land as a provision of access to recreation have been employed as proxies for natural recovery assessment. Costa Viera and Kerle (2014) used permeability of surfaces to measure environmental quality as a proxy for recovery assessment. For example, the permeability of impervious surfaces is very low in urban areas, which leads to an increase in flood frequency and storm flows, and consequently influences urban climate and pollution levels (Weng 2012). Similarly, low levels of permeability of surfaces increase the vulnerability of the area to water-related hazards (Kablan et al. 2017).

Disasters destroy the natural environment directly through landslide and flooding, or indirectly by human activities such as intense construction work after a disaster in the recovery stage (Brown et al. 2010a). Consequently, monitoring land cover provides a proxy information on the natural environment recovery, e.g. land cover change from tree/forest to building as a result of constructions during the recovery processes (Costa Viera and Kerle 2014; Wagner et al. 2012). Land cover change assessments also contribute to monitoring land degradation, erosion and deforestation in the recovery processes (Cebecauer and Hofierka 2008; Quan et al. 2011).

Land cover and land use types are also used to indicate the vulnerability of the natural environment (Guntur et al. 2017; Ntajal et al. 2017; Poompavai and Ramalingam 2013; Rimba et al. 2017; Taubenböck et al. 2008). For example, expansion of urbanized areas, cultivation development, and decreasing of natural forest lands increase the eco-environmental vulnerability to hazards (Liou et al. 2017). In another example, Mainali and Pricope (2017) associate built-up and bare land with high vulnerability ranks and forest land - with low natural vulnerability rank (Mainali and Pricope 2017). In addition, a high proportion of managed natural land cover indicates greater vulnerability because vegetation and crop yield are negatively influenced by environmental degradation (Yoo et al. 2014). 
Evapotranspiration is a combined process of evaporation and transpiration and is an important factor in the hydrological cycle. Consequently, it plays a role of a proxy to evaluate flood vulnerability through extracting vegetation cover and type (Liu et al. 2017b).

Frazier et al. (2013) use gross primary production (GPP) as a proxy for ecosystem-wellness derived from RS data, and assess the effect of resilience of ecological capital in DRM, particularly in recovery processes. GPP represents the photosynthetic capacity of vegetation, which can be used to estimate the vegetation productive capability. Liu et al. (2010) employ it also for damage and recovery assessments.

Ecological RS studies offer robust proxies applicable for urban DRM. For example, high plants productivity is directly related to good soil moisture and nutrient retention. The amount of soil organic carbon is a proxy to assess them and can be extracted using RS data (Bhunia et al. 2017; Rasel et al. 2017). Therefore, areas with higher soil organic carbon are more suitable for agriculture and consequently more resilient to disasters, particularly those linked to climate change (Lal 2006). 


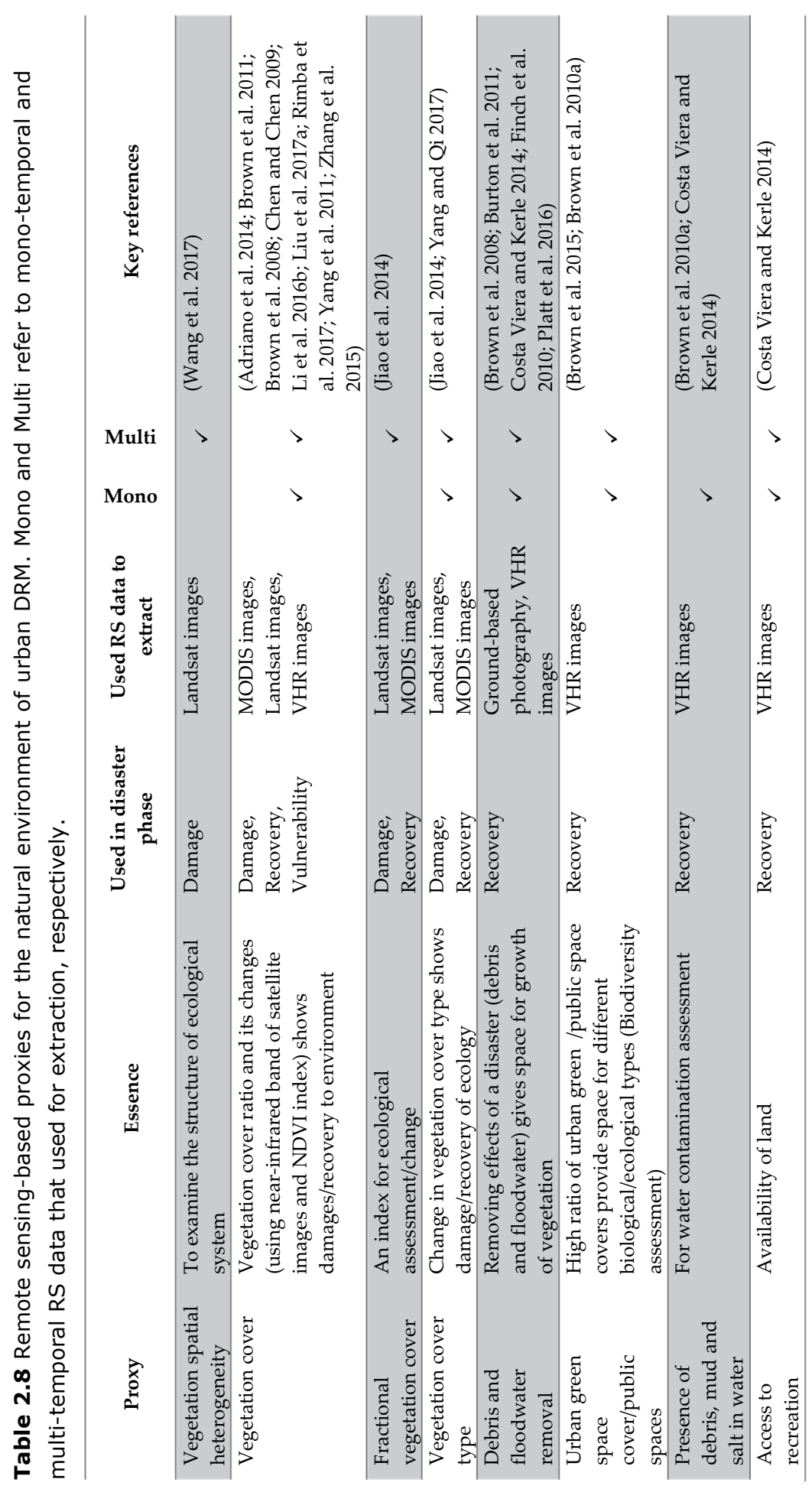




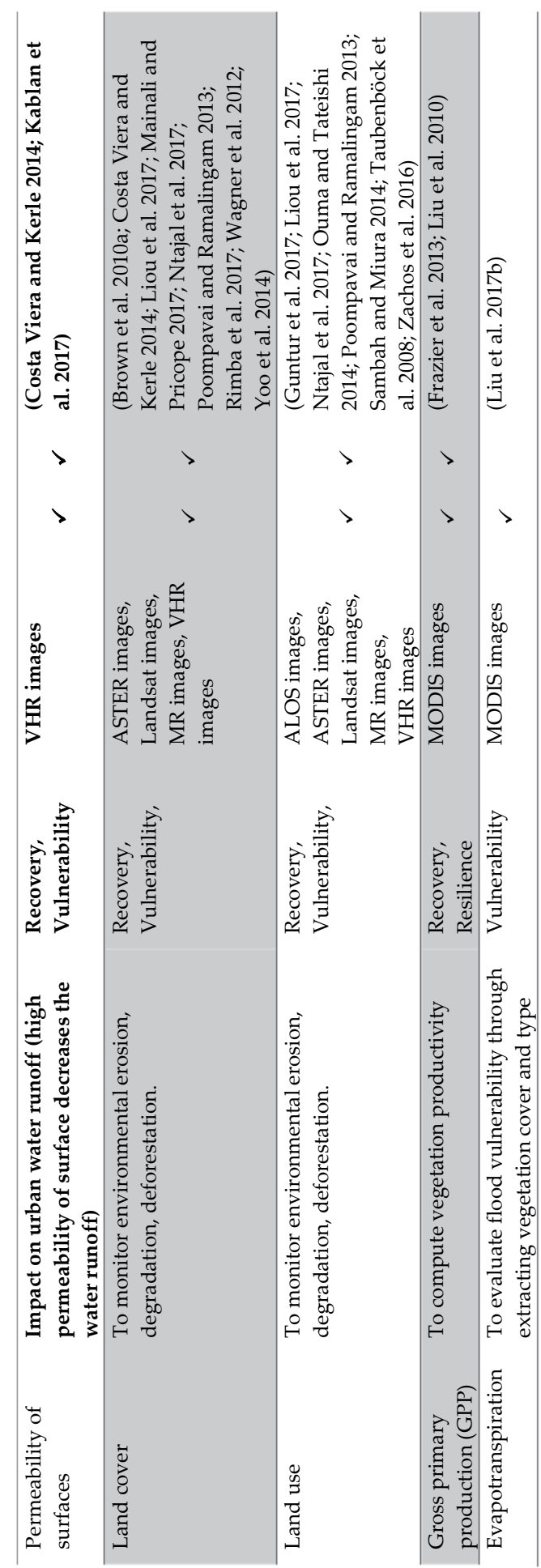




\subsection{Conclusions and discussion}

This article surveyed recent advances in the development of RS-based proxies for urban DRM, focusing on the literature for both pre- and post-disaster situation assessments. Specifically, damage, recovery, vulnerability, and resilience RS-based proxies were identified along the four dimensions of builtup, economic, social, and natural environments. The structured review of 109 published articles shows that a comprehensive toolkit of RS-proxies has been developed and is widely used in practice. We observed an increasing use in the number of proxy-based studies in urban DRM. This is due to the availability of diverse and detailed image data and rapidly increasing computational power to a growing number of communities. This also shows an increasing need for and interest in proxy-based measurements.

Despite significant progress, there are gaps evident in the field that demand further research to focus on a number of directions:

(1) A rich set of RS-based proxies currently focuses on the physical side of urban DRM. Yet, urban DRM assessments rarely go beyond aggregated proxies for the socio-economic environment and the offered proxies are very limited to assess functional aspects. For example, although a good number of proxies has been developed for building-based damage detection, none of them addresses the functionality of the buildings that is also a critical information source for all aspects of urban DRM. Assessing the extent of damage to socio-economic activities and their functional recovery is essential and will likely be possible via a complimentary set of proxies rather than a single index.

(2) RS-based proxies have been developed in several different fields that can be interchangeably used for other DRM phases with little modification in estimation methodology. There are relatively few detailed proxies for recovery assessment compared to damage assessment in built-up environments (Figure 2.2), whereas most of the damage assessment proxies can also be used for recovery assessment. The opposite is also true, and some of the RS-based proxies that have been developed for other environments, e.g. in the economics environment, can also be used for damage assessment. Furthermore, this statement is also correct with respect to the use of proxies for other risk elements in urban DRM: e.g. GPP that has been used for economic recovery and resilience assessments, could also be used for damage and vulnerability assessments. As a result, developing proxies focused on a particular risk element may overlook similar proxies, which have been developed to address the same point but under different names. Our review integrates these different streams of literature and offers a structured overview of the state-of-the-art RS methods across various DRM phases. More work on aligning methodological 
advancements and use of similar RS tools, data and analysis to address DRM at various stages is vital to advance the field without reinventing the wheel.

(3) Using insights from other disciplines in developing RS-based proxies offers possibilities to intelligently connect various proxies that allow to comprehensively assess vulnerability, resilience, damage and recovery for urban DRM beyond the physical impact alone. As suggested in this review, a structured analysis of specific proxy-based examples and finding important proxies from structural engineering, economic, social and natural sciences for urban DRM will be instrumental in creating a public interdisciplinary library of methods for urban DRM. If maintained and updated by an interdisciplinary scholarly and practitioners community, such an open library will always rely on the state-of-the-art RS data and processing methods to extract them. It will help the RS community to fill the gap in comprehensive assessments of the different DRM phases in urban settings and beyond.

(4) Disaster resilience is one of the significant components of DRM, which has been gaining increasing importance in this field. However, only seven of the reviewed articles focus on resilience (Figure 2.1), and only 7 RS-based proxies have been developed for its assessment (Figure 2.2). These numbers indicate a significant need for further studies in resilience assessments. Modifying and using RS-based proxies form other disciplines such as vulnerability, which is comprehensively discussed in this paper, can help the RS community to develop proxies for resilience assessments.

\subsection{References of Chapter 2}

Adriano, B., Gokon, H., Mas, E., Koshimura, S., Liu, W., Matsuoka, M., 2014. Extraction of damaged areas due to the 2013 haiyan typhoon using aster data. IEEE International Geoscience and Remote Sensing Symposium (pp. 2154-2157). New York: IEEE.

Allan, P., Bryant, M., 2011. Resilience as a framework for urbanism and recovery. Journal of Landscape Architecture, 34-45.

Arciniegas, G. A., Bijker, W., Kerle, N., Tolpekin, V. A., 2007. Coherence- and Amplitude-Based Analysis of Seismogenic Damage in Bam, Iran, Using ENVISAT ASAR Data. IEEE Transactions on Geoscience and Remote Sensing, 45(6), 1571-1581.

Armenakis, C., Nirupama, N., 2013. Prioritization of disaster risk in a community using GIS. Natural Hazards, 66(1), 15-29.

Aubrecht, C., Steinnocher, K., Hollaus, M., Wagner, W., 2009. Integrating earth observation and GIScience for high resolution spatial and functional modeling of urban land use. Computers, Environment and Urban Systems, $33(1), 15-25$.

Baiocchi, V., Zottele, F., Dominici, D., 2017. Remote Sensing of Urban Microclimate Change in L'Aquila City (Italy) after Post-Earthquake Depopulation in an Open Source GIS Environment. Sensors, 17(2), 12. 
Berke, P. R., J., K., D., W., 1993. Recovery after Disaster: Achieving Sustainable Development, Mitigation and Equity. Disasters, 17(2).

Bertinelli, L., Strobl, E., 2013. Quantifying the Local Economic Growth Impact of Hurricane Strikes: An Analysis from Outer Space for the Caribbean. Journal of Applied Meteorology and Climatology, 52(8), 1688-1697.

Bevington, J., Pyatt, S., Hill, A., Honey, M., Adams, B., Davidson, R., Brink, S., Chang, S., Panjwani, D., Mills, R., Amyx, P., Eguchi, R., 2010. Uncovering community disruption using remote sensing: An assessment of early recovery in post-earthquake Haiti. Miscellaneous Report \#69, University of Delaware (Newark, Delaware USA), Disaster Research Center.

Bhunia, G. S., Kumar Shit, P., Pourghasemi, H. R., 2017. Soil organic carbon mapping using remote sensing techniques and multivariate regression model. Geocarto International, 1-12.

Borfecchia, F., Pollino, M., De Cecco, L., Lugari, A., Martini, S., La Porta, L., Ristoratore, E., Pascale, C., 2010. Active and passive remote sensing for supporting the evaluation of the urban seismic vulnerability. Italian Journal of Remote Sensing, 42, 129-141.

Bradshow, S., 2004. Socio-economic impacts of natural disaster: a gender analysis. Nation Economic Commission for Latin America (ECLA), Serie Manuales 32.

Brown, D., Platt, S., Bevington, J., Saito, K., Adams, B., Chenvidyakarn, T., Spence, R., Chuenpagdee, R., Khan, A., 2015. Monitoring and evaluating post-disaster recovery using high-resolution satellite imagery - towards standardised indicators for post-disaster recovery. Cambridge University for Risk in the Built Environment, University of Cambridge: Cambridge, UK, 2010.

Brown, D., S., P., J., B., 2010a. Disaster recovery indicators: Guidlines for monitoring and evaluation. University of Cambridge: Cambridge, UK: CURBE, Cambridge University for Risk in the Built Environment.

Brown, D., Saito, K., Liu, M., Spence, R., So, E., Ramage, M., 2011. The use of remotely sensed data and ground survey tools to assess damage and monitor early recovery following the 12.5.2008 Wenchuan earthquake in China. Bulletin of Earthquake Engineering, 10(3), 741-764.

Brown, D., Saito, K., Spence, R., T., C., 2008. Indicators for measuring, monitoring and evaluating post-disaster recovery. Paper presented at the 6th International Workshop on Remote Sensing for Disaster Applications. Cambridge University for Risk in the Built Environment, University of Cambridge: Cambridge, UK.

Brown, D., Saito, K., T., C., 2010b. Monitoring and evaluating post disaster recovery using high resolution satellite imagery. Cambridge University for Risk in the Built Environment, University of Cambridge: Cambridge, UK, 2010.

Buehler, Y. A., Kellenberger, T. W. , Small, D., Itten, K. I., 2006. Rapid mapping with remote sensing data during flooding 2005 in Switzerland by objectbased methods: a case study. In J. F. Martin-Duque, C. A. Brebbia, D. E. Emmanouloudis, \& U. Mander (Eds.), Geo-Environment and Landscape Evolution Ii: Evolution, Monitoring, Simulation, Management and Remediation of the Geological Environment and Landscape (Vol. 89, pp. 391-+). Southampton: Wit Press.

Burton, C., Mitchell, J. T., Cutter, S. L., 2011. Evaluating post-Katrina recovery in Mississippi using repeat photography. Disasters, 35(3), 488-509. 
Burton, C. G., 2012. The development of metrics for community resilience to Natural disasters. University of South Carolina, University of South Carolina.

Burton, C. G., 2014. A validation of metrics for community resilience to natural hazards and disasters using the recovery from hurricane Katrina as a case study. Annals of the Association of American Geographers, 105(1), 67-86.

Cebecauer, T., Hofierka, J., 2008. The consequences of land-cover changes on soil erosion distribution in Slovakia. Geomorphology, 98(3-4), 187-198.

Chang, S. E., Adams, B. J., Alder, J., Berke, P. R., Chuenpagdee, R., Ghosh, S., Wabnitz, C., 2006. Coastal Ecosystems and Tsunami Protection after the December 2004 Indian Ocean Tsunami. Earthquake Spectra, 22(S3), 863-887.

Chen, P., Chen, X. L., 2009. Remote sensing and GIS-based Flood Vulnerability Assessment in Jiangzi Province in China. Los Alamitos: IEEE Computer Soc.

Chen, S. C., Shyu, M. L., Zhang, C. C., Tang, W. Z., Zhang, K. Q., 2003. Damage pattern mining in hurricane image databases. New York: IEEE.

Contreras, D., Blaschke, T., Hodgson, M. E., 2017. Lack of spatial resilience in a recovery process: Case L'Aquila, Italy. Technological Forecasting and Social Change, 121, 76-88.

Contreras, D., Blaschke, T., Kienberger, S., Zeil, P., 2013. Spatial connectivity as a recovery process indicator: The L'Aquila earthquake. Technological Forecasting and Social Change, 80(9), 1782-1803.

Contreras, D., Blaschke, T., Kienberger, S., Zeil, P., 2014. Myths and realities about the recovery of L'Aquila after the earthquake. International Journal of Disaster Risk Reduction, 8, 125-142.

Contreras, D., Blaschke, T., Tiede, D., Jilge, M., 2016. Monitoring recovery after earthquakes through the integration of remote sensing, GIS, and ground observations: the case of L'Aquila (Italy). Cartography and Geographic Information Science, 43(2), 115-133.

Cooner, A., Shao, Y., Campbell, J., 2016. Detection of Urban Damage Using Remote Sensing and Machine Learning Algorithms: Revisiting the 2010 Haiti Earthquake. Remote Sensing, 8(10), 868.

Coppola, D. P., 2015. Chapter 1 - The Management of Disasters Introduction to International Disaster Management (Third Edition) (pp. 1-39). Boston: Butterworth-Heinemann.

Costa Viera, A., Kerle, N., 2014. Utility of geo-informatics for disaster risk management: Linking structural damage assessment, recovery and resilience. University of Twente, The Netherlands.

Costanzo, A., Montuori, A., Silva, J., Silvestri, M., Musacchio, M., Doumaz, F., Stramondo, S., Buongiorno, M., 2016. The Combined Use of Airborne Remote Sensing Techniques within a GIS Environment for the Seismic Vulnerability Assessment of Urban Areas: An Operational Application. Remote Sensing, 8(2), 146.

Cox, A., Prager, F., Rose, A., 2011. Transportation security and the role of resilience: A foundation for operational metrics. Transport Policy, 18(2), 307-317.

Cusicanqui, J., Kerle, N., Nex, F., 2018. Usability of aerial video footage for 3D-scene reconstruction and structural damage assessment. Natural Hazards and Earth System Sciences Discussions, 1-23. 
Cutter, S. L., 2016. The landscape of disaster resilience indicators in the USA. Natural Hazards, 80(2), 741-758.

Cutter, S. L., Ash, K. D., Emrich, C. T., 2016. Urban-Rural Differences in Disaster Resilience. Annals of the American Association of Geographers, 106(6), 1236-1252.

Cutter, S. L., Barnes, L., Berry, M., Burton, C., Evans, E., Tate, E., Webb, J., 2008. A place-based model for understanding community resilience to natural disasters. Global Environmental Change, 18(4), 598-606.

Cutter, S. L., Burton, C. G., Emrich, C. T., 2010. Disaster Resilience Indicators for Benchmarking Baseline Conditions. Journal of Homeland Security and Emergency Management, 7(1).

De Almeida, L. Q., Welle, T., Birkmann, J., 2016. Disaster risk indicators in Brazil: A proposal based on the world risk index. International Journal of Disaster Risk Reduction, 17, 251-272.

Dong, L., Shan, J., 2013. A comprehensive review of earthquake-induced building damage detection with remote sensing techniques. ISPRS Journal of Photogrammetry and Remote Sensing, 84, 85-99.

Du, P., Liu, P., Xia, J., Feng, L., Liu, S., Tan, K., Cheng, L., 2014. Remote Sensing Image Interpretation for Urban Environment Analysis: Methods, System and Examples. Remote Sensing, 6(10), 9458-9474.

Duarte, D., Nex, F., Kerle, N., Vosselman, G., 2018. Satellite image classification of building damages using airborne and satellite image samples in a deep learning approach. ISPRS Annals of Photogrammetry, Remote Sensing and Spatial Information Sciences, IV-2, 89-96.

Ebert, A., Kerle, N., Stein, A., 2009. Urban social vulnerability assessment with physical proxies and spatial metrics derived from air- and spaceborne imagery and GIS data. Natural Hazards, 48(2), 275-294.

Elliott, R. J. R., Strobl, E., Sun, P. Y., 2015. The local impact of typhoons on economic activity in China: A view from outer space. Journal of Urban Economics, 88, 50-66.

Engineering, N. a. O., 2013. Harnessing Operational Systems Engineering to Support Peacebuilding: Report of a Workshop by the National Academy of Engineering and United States Institute of Peace Roundtable on Technology, Science, and Peacebuilding. Washington, DC: The National Academies Press.

Fatemi, F., Ardalan, A., Aguirre, B., Mansouri, N., Mohammadfam, I., 2017. Social vulnerability indicators in disasters: Findings from a systematic review. International Journal of Disaster Risk Reduction, 22, 219-227.

Feng, T. N., Hong, Z. H., Wu, H. J., Fu, Q. S., Wang, C. X., Jiang, C. H., Tong, X. H., 2013. Estimation of earthquake casualties using high-resolution remote sensing: a case study of Dujiangyan city in the May 2008 Wenchuan earthquake. Natural Hazards, 69(3), 1577-1595.

Ferreira, T. M., Vicente, R., Varum, H., 2014. Seismic vulnerability assessment of masonry facade walls: development, application and validation of a new scoring method. Structural Engineering and Mechanics, 50(4), 541-561.

Finch, C., Emrich, C. T., Cutter, S. L., 2010. Disaster disparities and differential recovery in New Orleans. Population and Environment, 31(4), 179-202.

Flax, L., Jakson, R., Stein, D., 2002. Community vulnerability assessment tool methodology. Nat. Hazards Rev., 3, 163-176. 
Frazier, A. E., Renschler, C. S., Miles, S. B., 2013. Evaluating post-disaster ecosystem resilience using MODIS GPP data. International Journal of Applied Earth Observation and Geoinformation, 21, 43-52.

Friedland, C., Massarra, C., Henderson, E., 2011. Integrated Aerial-Based and Ground-Based Damage Assessment of Single Family Dwellings at the Neighborhood and Per-Building Spatial Scales. Paper presented at the 9th International Workshop on Remote Sensing for Disaster Response, Stanford, CA.

Galarreta, J. F., 2014. Urban structural damage assessment using objectoriented analysis and semantic reasoning. (MSc), University of Twente.

Galarreta, J. F., Kerle, N., Gerke, M., 2015. UAV-based urban structural damage assessment using object-based image analysis and semantic reasoning. Natural Hazards and Earth System Sciences, 15(6), 10871101.

Gao, F., Zhang, L., Wang, J., Mei, J., 2015. Change Detection in Remote Sensing Images of Damage Areas with Complex Terrain Using Texture Information and SVM. Paper presented at the International Conference on Circuits and Systems (CAS 2015).

Geiß, C., Aravena Pelizari, P., Marconcini, M., Sengara, W., Edwards, M., Lakes, T., Taubenböck, H., 2015. Estimation of seismic building structural types using multi-sensor remote sensing and machine learning techniques. ISPRS Journal of Photogrammetry and Remote Sensing, 104, 175-188.

Geiß, C., Jilge, M., Lakes, T., Taubenbock, H., 2016. Estimation of Seismic Vulnerability Levels of Urban Structures With Multisensor Remote Sensing. Ieee Journal of Selected Topics in Applied Earth Observations and Remote Sensing, 9(5), 1913-1936.

Gerke, M., Kerle, N., 2011. Automatic Structural Seismic Damage Assessment with Airborne Oblique Pictometry(C) Imagery. Photogrammetric Engineering \& Remote Sensing, 77(9), 885-898.

Gil, J., Steinbach, P., 2008. From flood risk to indirect flood impact: evaluation of street network performance for effective management, response and repair. In D. Proverbs, C. A. Brebbia, \& E. PenningRowsell (Eds.), Flood Recovery, Innovation and Response (Vol. 118, pp. 335-344). Southampton: Wit Press.

Guntur, Sambah, A. B., Miura, F., Fuad, Arisandi, D. M., 2017. Assessing tsunami vulnerability areas using satellite imagery and weighted cellbased analysis. International Journal of Geomate, 12(34), 115-122.

Hagelman, R. R., Connolly, M. H., Zavar, E. M., Dahal, K. R., 2012. Disaster reconstruction and business geography following the 2007 Greensburg, Kansas Tornado. Environmental Hazards, 11(4), 283-302.

Hoque, M. a.-A., Phinn, S., Roelfsema, C., Childs, I., 2017. Tropical cyclone disaster management using remote sensing and spatial analysis: A review. International Journal of Disaster Risk Reduction, 22, 345-354.

Hsieh, C. H., Feng, C. M., 2014. Road network vulnerability assessment based on fragile factor interdependencies in spatial-functional perspectives. Environment and Planning A, 46(3), 700-714.

Hu, S. S., Cheng, X. J., Zhou, D. M., Zhang, H., 2017. GIS-based flood risk assessment in suburban areas: a case study of the Fangshan District, Beijing. Natural Hazards, 87(3), 1525-1543. 
Huang, W., Li, S., 2016. Understanding human activity patterns based on space-time-semantics. ISPRS Journal of Photogrammetry and Remote Sensing, 121, 1-10.

Huang, X., Zhang, L., 2012. Morphological Building/Shadow Index for Building Extraction From High-Resolution Imagery Over Urban Areas. Ieee Journal of Selected Topics in Applied Earth Observations and Remote Sensing, 5(1), 161-172.

Ilehag, R., Schenk, A., Hinz, S., 2017. Concept for classifying facade elements based on material, geometry and thermal radiation using multimodal uav remote sensing. Int. Arch. Photogramm. Remote Sens. Spatial Inf. Sci., $X L I I-2 / W 6,145-151$.

Jafari, N., Shahsanai, A., Memarzadeh, M., Loghmani, A., 2011. Prevention of communicable diseases after disaster: A review. Journal of Research in Medical Sciences : The Official Journal of Isfahan University of Medical Sciences, 16(7), 956-962.

Janalipour, M., Taleai, M., 2016. Building change detection after earthquake using multi-criteria decision analysis based on extracted information from high spatial resolution satellite images. International Journal of Remote Sensing, 38(1), 82-99.

Jiang, S., Friedland, C. J., 2015. Automatic urban debris zone extraction from post-hurricane very high-resolution satellite and aerial imagery. Geomatics, Natural Hazards and Risk, 7(3), 933-952.

Jiao, Q. J., Zhang, B., Liu, L. Y., Li, Z. W., Yue, Y. M., Hu, Y., 2014. Assessment of spatio-temporal variations in vegetation recovery after the Wenchuan earthquake using Landsat data. Natural Hazards, 70(2), 1309-1326.

Kablan, M. K. A., Dongo, K., Coulibaly, M., 2017. Assessment of Social Vulnerability to Flood in Urban Cote d'Ivoire Using the MOVE Framework. Water, 9(4), 19.

Kerle, N., Hoffman, R. R., 2013. Collaborative damage mapping for emergency response: The role of cognitive systems engineering. Natural Hazards and Earth System Science, 13(1), 97-113.

Koshimura, S., Hayashi, S., Gokon, H., 2014. The impact of the 2011 Tohoku earthquake tsunami disaster and implications to the reconstruction. Soils and Foundations, 54(4), 560-572.

Kotzee, I., Reyers, B., 2016. Piloting a social-ecological index for measuring flood resilience: A composite index approach. Ecological Indicators, 60, 45-53.

Kubal, C., Haase, D., Meyer, V., Scheuer, S., 2009. Integrated urban flood risk assessment - adapting a multicriteria approach to a city. Natural Hazards and Earth System Sciences, 9(6), 1881-1895.

Kuffer, M., Pfeffer, K., Sliuzas, R., 2016. Slums from Space-15 Years of Slum Mapping Using Remote Sensing. Remote Sensing, 8(6), 455.

Kumagai, K., 2012. Spatial comparison between densely built-up districts from the viewpoint of vulnerability to road blockades with respect to evacuation behavior. In M. Shortis, J. Shi, \& E. Guilbert (Eds.), Xxii Isprs Congress, Technical Commission Ii (Vol. 39-B2, pp. 151-156). Gottingen: Copernicus Gesellschaft Mbh.

Lal, R., 2006. Enhancing crop yields in the developing countries through restoration of the soil organic carbon pool in agricultural lands. Land Degradation \& Development, 17(2), 197-209. 
Li, M., Stein, A., Bijker, W., Zhan, Q., 2016a. Urban land use extraction from Very High Resolution remote sensing imagery using a Bayesian network. ISPRS Journal of Photogrammetry and Remote Sensing, 122, 192-205.

Li, M. Y., Wu, W., Wang, J., Che, Z. M., Xie, Y. L., 2017. Simulating and mapping the risk of surge floods in multiple typhoon scenarios: A case study of Yuhuan County, Zhejiang Province, China. Stochastic Environmental Research and Risk Assessment, 31(3), 645-659.

Li, S. D., Tang, H., He, S., Shu, Y., Mao, T., Li, J., Xu, Z. H., 2015. Unsupervised Detection of Earthquake-Triggered Roof-Holes From UAV Images Using Joint Color and Shape Features. IEEE Geoscience and Remote Sensing Letters, 12(9), 1823-1827.

Li, X. C., Yu, L., Xu, Y. D., Yang, J., Gong, P., 2016b. Ten years after Hurricane Katrina: monitoring recovery in New Orleans and the surrounding areas using remote sensing. Science Bulletin, 61(18), 1460-1470.

Liou, Y. I. A., Nguyen, A. K., Li, M. H., 2017. Assessing spatiotemporal ecoenvironmental vulnerability by Landsat data. Ecological Indicators, 80, 5265.

Liu, J., Gong, J. H., Liang, J. M., Li, Y., Kang, L. C., Song, L. L., Shi, S. X., 2017a. A quantitative method for storm surge vulnerability assessment a case study of Weihai city. International Journal of Digital Earth, 10(5), 539-559.

Liu, J., Shi, Z. W., 2017. Quantifying land-use change impacts on the dynamic evolution of flood vulnerability. Land Use Policy, 65, 198-210.

Liu, L., Jing, X., Wang, J., Zhao, C., 2009. Analysis of the changes of vegetation coverage of western Beijing mountainous areas using remote sensing and GIS. Environ Monit Assess, 153(1-4), 339-349.

Liu, R., Chen, Y., Wu, J. P., Gao, L., Barrett, D., Xu, T. B., Li, X. J., Li, L. Y., Huang, C., Yu, J., 2017b. Integrating Entropy-Based Naive Bayes and GIS for Spatial Evaluation of Flood Hazard. Risk Analysis, 37(4), 756-773.

Liu, Y., Liu, R., Ge, Q., 2010. Evaluating the vegetation destruction and recovery of Wenchuan earthquake using MODIS data. Natural Hazards, 54(3), 851-862.

Mainali, J., Pricope, N. G., 2017. High-resolution spatial assessment of population vulnerability to climate change in Nepal. Applied Geography, 82, 66-82.

Maruyama, Y., Tashiro, A., Yamazaki, F., 2011. Use of Digital Surface Model Constructed from Digital Aerial Images to Detect Collapsed Buildings during Earthquake. Procedia Engineering, 14, 552-558.

Mccarthy, M. J., Colna, K. E., El-Mezayen, M. M., Laureano-Rosario, A. E., Mendez-Lazaro, P., Otis, D. B., Toro-Farmer, G., Vega-Rodriguez, M., Muller-Karger, F. E., 2017. Satellite Remote Sensing for Coastal Management: A Review of Successful Applications. Environ. Manage., $60(2), 323-339$.

MCDEM. 2005. Focus on Recovery: A Holistic Framework for Recovery in New Zealand. MCDEM, New Zealand.

Mellander, C., Lobo, J., Stolarick, K., Matheson, Z., 2015. Night-Time Light Data: A Good Proxy Measure for Economic Activity? Plos One, 10(10), e0139779.

Miura, H., Midorikawa, S., Kerle, N., 2013. Detection of Building Damage Areas of the 2006 Central Java, Indonesia, Earthquake through Digital Analysis of Optical Satellite Images. Earthquake Spectra, 29(2), 453-473. 
Mohan, P., Strobl, E., 2017. The short-term economic impact of tropical Cyclone Pam: an analysis using VIIRS nightlight satellite imagery. International Journal of Remote Sensing, 38(21), 5992-6006.

Montgomery, M., Gragnolati, M., Burke, K., Paredes, E., 2000. Measuring living standards with proxy variables. Demography, 37(2), 155-174.

Morrow, B. H., 1999. Identifying and Mapping Community Vulnerability. Disasters, 23(1), 1-18.

Mück, M., Taubenböck, H., Post, J., Wegscheider, S., Strunz, G., Sumaryono, S., Ismail, F. A., 2012. Assessing building vulnerability to earthquake and tsunami hazard using remotely sensed data. Natural Hazards, 68(1), 97114.

Mueller, M., Segl, K., Heiden, U., Kaufmann, H., 2006. Potential of HighResolution Satellite Data in the Context of Vulnerability of Buildings. Natural Hazards, 38(1-2), 247-258.

Müller, A., 2013. Flood risks in a dynamic urban agglomeration: a conceptual and methodological assessment framework. Natural Hazards, 65(3), 1931-1950.

Müller, A., Reiter, J., Weiland, U., 2011. Assessment of urban vulnerability towards floods using an indicator-based approach - a case study for Santiago de Chile. Natural Hazards and Earth System Science, 11(8), 2107-2123.

Norris, F. H., Stevens, S. P., Pfefferbaum, B., Wyche, K. F., Pfefferbaum, R. L., 2008. Community resilience as a metaphor, theory, set of capacities, and strategy for disaster readiness. Am. J. Community Psychol., 41(1-2), 127150.

Ntajal, J., Lamptey, B. L., Mahamadou, I. B., Nyarko, B. K., 2017. Flood disaster risk mapping in the Lower Mono River Basin in Togo, West Africa. International Journal of Disaster Risk Reduction, 23, 93-103.

O'neill, J.R. Krummel, R.H. Gardner, G. Sugihara, B. Jackson, D.L. Deangelis, B.T. Milne, M.G. Turner, B. Zygmunt, S.W. Christensen, V.H. Dale, Graham, R. L., 1988. Indices of landscape pattern. Landscape Ecology, 1(3), 153-162.

Ouma, Y. O., Tateishi, R., 2014. Urban flood vulnerability and risk mapping using integrated multi-parametric AHP and GIS: Methodological overview and case study assessment. Water, 6(6), 1515-1545.

Pallard, B., Castellarin, A., Montanari, A., 2009. A look at the links between drainage density and flood statistics. Hydrology and Earth System Sciences, 13, 1019-1029.

Patino, C. L., Saripada, N. A., Olavides, R. D., Sinogaya, J., 2016. Running to safety: Analysis of disaster susceptibility of neighborhoods and proximity of safety facilities in Silay city, Philippines. In L. Halounova, V. Safar, P. L. N. Raju, L. Planka, V. Zdimal, T. S. Kumar, F. S. Faruque, Y. Kerr, S. M. Ramasamy, J. Comiso, Y. A. Hussin, P. S. Thenkabail, S. Lavender, A. Skidmore, P. Yue, P. Patias, O. Altan, \& Q. Weng (Eds.), Xxiii Isprs Congress, Commission Viii (Vol. 41, pp. 139-143). Gottingen: Copernicus Gesellschaft Mbh.

Penchev, G., 2016. Using space syntax for estimation of potential disaster indirect economic losses. Comparative Economic Research-Central and Eastern Europe, 19(5), 125-142.

Pidwirny, M., 2017. Glossary of terms for physical geography. Our Planet Earth Publishing: Kelowna, BC, Canada. 
Pitts, D. a. D., So, E., 2017. Enhanced change detection index for disaster response, recovery assessment and monitoring of accessibility and open spaces (camp sites). International Journal of Applied Earth Observation and Geoinformation, 57, 49-60.

Platt, S., Brown, D., Hughes, M., 2016. Measuring resilience and recovery. International Journal of Disaster Risk Reduction, 19, 447-460.

Poompavai, V., Ramalingam, M., 2013. Geospatial Analysis for Coastal Risk Assessment to Cyclones. Journal of the Indian Society of Remote Sensing, 41(1), 157-176.

Prince, D., Sidike, P., Essa, A., Asari, V., 2017. Multifeature fusion for automatic building change detection in wide-area imagery. SPIE:Washington, DC, USA, 2017; p. 21.

Proville, J., Zavala-Araiza, D., Wagner, G., 2017. Night-time lights: A global, long term look at links to socio-economic trends. Plos One, 12(3), e0174610.

Qasim, S., Qasim, M., Shrestha, R. P., Khan, A. N., Tun, K., Ashraf, M., 2016. Community resilience to flood hazards in Khyber Pukhthunkhwa province of Pakistan. International Journal of Disaster Risk Reduction, 18, 100-106.

Quan, B., Römkens, M. J. M., Li, R., Wang, F., Chen, J., 2011. Effect of land use and land cover change on soil erosion and the spatio-temporal variation in Liupan Mountain Region, southern Ningxia, China. Frontiers of Environmental Science \& Engineering in China, 5(4), 564-572.

Rasel, S. M. M., Groen, T. A., Hussin, Y. A., Diti, I. J., 2017. Proxies for soil organic carbon derived from remote sensing. International Journal of Applied Earth Observation and Geoinformation, 59, 157-166.

Rastiveis, H., Eslamizade, F., Hosseini-Zirdoo, E., 2015. Building damage assessment after earthquake using post-event LiDAR data. ISPRS International Archives of the Photogrammetry, Remote Sensing and Spatial Information Sciences, $X L-1-W 5,595-600$.

Rastiveis, H., Samadzadegan, F., Reinartz, P., 2013. A fuzzy decision making system for building damage map creation using high resolution satellite imagery. Natural Hazards and Earth System Science, 13(2), 455-472.

Rezaie, F., Panahi, M., 2015. GIS modeling of seismic vulnerability of residential fabrics considering geotechnical, structural, social and physical distance indicators in Tehran using multi-criteria decision-making techniques. Natural Hazards and Earth System Sciences, 15(3), 461-474.

Rimba, A., Setiawati, M., Sambah, A., Miura, F., 2017. Physical Flood Vulnerability Mapping Applying Geospatial Techniques in Okazaki City, Aichi Prefecture, Japan. Urban Science, 1(1), 7.

Rose, A., Krausmann, E., 2013. An economic framework for the development of a resilience index for business recovery. International Journal of Disaster Risk Reduction, 5, 73-83.

Roy, D. C., Blaschke, T., 2015. Spatial vulnerability assessment of floods in the coastal regions of Bangladesh. Geomatics Natural Hazards \& Risk, 6(1), 21-44.

Rubin, C., Spence, R., Going, C., Markus, M., 1985. Community recovery from a major natural disaster. Institute of Behavioral science University of Colorado Boulder, co, Monograph 41.

Saito, K., Spence, R. J. S., Going, C., Markus, M., 2004. Using High-Resolution Satellite Images for Post-Earthquake Building Damage Assessment: A 
Study Following the 26 January 2001 Gujarat Earthquake. Earthquake Spectra, 20(1), 145-169.

Samadzadegan, F., Rastiveisi, H., 2008. Automatic detection and classification of damaged buildings, using high resolution satellite imagery and vector data. Paper presented at the International Archives of Photogrammetry Remote Sensing and Spatial Information Sciences.

Sambah, A., Miura, F., 2014. Remote sensing and spatial multi-criteria analysis for tsunami vulnerability assessment. Disaster Prevention and Management, 23(3), 271-295.

Sang, N., Chen, X., Nie, J., Yang, S., Fan, Y., Wen, Q., Xu, F., Li, L., 2015. Building losses assessment for Lushan earthquake utilization multisource remote sensing data and GIS. 9812, 98120J.

Sastry, N., Gregory, J., 2014. The Location of displaced New Orleans residents in the year after Hurricane Katrina. Demography, 51(3), 753-775.

Sghaier, M. O., Lepage, R., 2015, 26-31 July 2015. Road damage detection from VHR remote sensing images based on multiscale texture analysis and dempster shafer theory. Paper presented at the 2015 IEEE International Geoscience and Remote Sensing Symposium (IGARSS).

Song, Y., Knaap, G.-J., 2004. Measuring Urban Form: Is Portland winning the war on sprawl? Journal of the American Planning Association, 70(2), 210225.

Sowmya, K., John, C. M., Shrivasthava, N. K., 2015. Urban flood vulnerability zoning of Cochin City, southwest coast of India, using remote sensing and GIS. Natural Hazards, 75(2), 1271-1286.

Stefanidis, S., Stathis, D., 2013. Assessment of flood hazard based on natural and anthropogenic factors using analytic hierarchy process (AHP). Natural Hazards, 68(2), 569-585.

Stow, D., Lopez, A., Lippitt, C., Hinton, S., Weeks, J., 2007. Object-based classification of residential land use within Accra, Ghana based on QuickBird satellite data. Int. J. Remote Sens., 28(22), 5167-5173.

Stumpf, A., Kerle, N., 2011. Object-oriented mapping of landslides using Random Forests. Remote Sensing of Environment, 115(10), 2564-2577.

Sumaryono, S., 2010. Assessing Building Vulnerability to Tsunami Hazard Using Integrative Remote Sensing and GIS Approaches. (Dissertation, LMU Munich). Retrieved from http://nbnresolving.de/urn:nbn:de:bvb:19-123909

Tamkuan, N., Nagai, M., 2017. Fusion of Multi-Temporal Interferometric Coherence and Optical Image Data for the 2016 Kumamoto Earthquake Damage Assessment. ISPRS International Journal of Geo-Information, 6(7), 188.

Taubenböck, H., Post, J., Roth, A., Zosseder, K., Strunz, G., Dech, S., 2008. A conceptual vulnerability and risk framework as outline to identify capabilities of remote sensing. Natural Hazards and Earth Sytem Sciences, 8, 409-420.

Taubenböck, H., Wurm, M., Post, J., Roth, A., Strunz, G., Dech, S., 2009. Vulnerability assessment towards tsunami threats using multisensoral remote sensing data. In U. Michel \& D. L. Civco (Eds.), Remote Sensing for Environmental Monitoring, Gis Applications, and Geology Ix (Vol. 7478). Bellingham: Spie-Int Soc Optical Engineering.

The World Bank. 2014. Resilience indicators: Summary Note and Steps Forward. TheWorld Bank: Washington, DC, USA, 2014. 
Tian, J., Metzlaff, L., D’angelo, P., Reinartz, P., 2017. Region-Based Building Rooftop Extraction and Change Detection. ISPRS - International Archives of the Photogrammetry, Remote Sensing and Spatial Information Sciences, XLII-2/W7, 903-908.

Tu, J., Li, D., Feng, W., Han, Q., Sui, H., 2017a. Detecting Damaged Building Regions Based on Semantic Scene Change from Multi-Temporal HighResolution Remote Sensing Images. ISPRS International Journal of GeoInformation, 6(5), 131.

Tu, J., Sui, H., Feng, W., Song, Z., 2016. Automatic Building Damage Detection Method Using High-Resolution Remote Sensing Images and 3d Gis Model. ISPRS Annals of Photogrammetry, Remote Sensing and Spatial Information Sciences, III-8, 43-50.

Tu, J. H., Sui, H. G., Feng, W. Q., Sun, K. M., Xu, C., Han, Q. H., 2017b. Detecting building facade damage from oblique aerial images using local symmetry feature and the Gini Index. Remote Sensing Letters, 8(7), 676685.

Turker, M., Sumer, E., 2008. Building based damage detection due to earthquake using the watershed segmentation of the post event aerial images. International Journal of Remote Sensing, 29(11), 3073-3089.

UNISDR. 2009. Terminology on disaster risk reduction. United Nations International Strategy for Disaster Reduction (UNISDR), Geneva, Switzerland.

United Nations Department of Economic and Social Affairs., 2015. World Urbanization Prospects: The 2014 Revision. UNDESA: New York, NY, USA, 2015.

Upton, G., Cook, I., 2014. Oxford dictionary of statistics: Oxford university press.

Vetrivel, A., Gerke, M., Kerle, N., Nex, F., Vosselman, G., 2017. Disaster damage detection through synergistic use of deep learning and 3D point cloud features derived from very high resolution oblique aerial images, and multiple-kernel-learning. ISPRS Journal of Photogrammetry and Remote Sensing.

Vetrivel, A., Gerke, M., Kerle, N., Vosselman, G., 2015. Identification of damage in buildings based on gaps in 3D point clouds from very high resolution oblique airborne images. ISPRS Journal of Photogrammetry and Remote Sensing, 105, 61-78.

Vetrivel, A., Gerke, M., Kerle, N., Vosselman, G., 2016a. Identification of structurally damaged areas in airborne oblique images using a visual-bagof-words approach. Remote Sensing, 8(3), 231.

Vetrivel, A., Kerle, N., Gerke, M., Nex, F., Vosselman, G., 2016b. Towards automated satellite image segmentation and classification for assessing disaster damage using data specific features with incremental learning. Paper presented at the GEOBIA 2016, Enschede, The Netherlands.

Villagra, P., Rojas, C., Ohno, R., Xue, M., Gomez, K., 2014. A GIS-base exploration of the relationships between open space systems and urban form for the adaptive capacity of cities after an earthquake: The cases of two Chilean cities. Applied Geography, 48, 64-78.

Wagner, M. A., Myint, S. W., Cerveny, R. S., 2012. Geospatial assessment of recovery rates following a tornado disaster. IEEE Transactions on Geoscience and Remote Sensing, 50(11), 4313-4322. 
Wang, L., Dou, A. X., Wang, X. Q., Dong, Y. F., Ding, X., Li, Z., Yuan, X. X., Qiu, Y. R., 2011. Damage Assessment of Haiti Earthquake Emergency Using High Resolution Remote Sensing Imagery. In Q. Tong, X. Gu, \& B. Zhu (Eds.), Remote Sensing of the Environment: The 17th China Conference on Remote Sensing (Vol. 8203). Bellingham: Spie-Int Soc Optical Engineering.

Wang, L., Tian, B. W., Koike, K., Hong, B. T., Ren, P., 2017. Integration of Landscape Metrics and Variograms to Characterize and Quantify the Spatial Heterogeneity Change of Vegetation Induced by the 2008 Wenchuan Earthquake. ISPRS International Journal of Geo-Information, $6(6), 13$.

Weng, Q., 2012. Remote sensing of impervious surfaces in the urban areas: Requirements, methods, and trends. Remote Sensing of Environment, 117, 34-49.

Xian, S., Lin, N., Hatzikyriakou, A., 2015. Storm surge damage to residential areas: a quantitative analysis for Hurricane Sandy in comparison with FEMA flood map. Natural Hazards, 79(3), 1867-1888.

Yan, B. Y., Li, S. S., Wang, J., Ge, Z. M., Zhang, L. Q., 2016. Socio-economic vulnerability of the megacity of Shanghai (China) to sea-level rise and associated storm surges. Regional Environmental Change, 16(5), 14431456.

Yang, C., Ren, X., Huang, H., 2011. The vegetation damage assessment of the Wenchuan earthquake of May 2008 using remote sensing and GIS. Natural Hazards, 62(1), 45-55.

Yang, W. T., Qi, W. W., 2017. Spatial-Temporal Dynamic Monitoring of Vegetation Recovery After the Wenchuan Earthquake. Ieee Journal of Selected Topics in Applied Earth Observations and Remote Sensing, 10(3), 868-876.

Ye, J. Y., Lin, G. F., Zhang, M. F., Lin, Z. L., Chen, J. M., 2010. GIS-Based Study of Natural Disaster Vulnerability for Xiamen City. 2010 18th International Conference on Geoinformatics, 5.

Ye, X., Qin, Q., Liu, M., Wang, J., Wang, J., 2014, 13-18 July 2014. Building damage detection from post-quake remote sensing image based on fuzzy reasoning. Paper presented at the 2014 IEEE Geoscience and Remote Sensing Symposium.

Yoo, G., Kim, A. R., Hadi, S., 2014. A methodology to assess environmental vulnerability in a coastal city: Application to Jakarta, Indonesia. Ocean \& Coastal Management, 102, 169-177.

Yu, B., Liu, H., Wu, J., Hu, Y., Zhang, L., 2010. Automated derivation of urban building density information using airborne LiDAR data and object-based method. Landscape and Urban Planning, 98(3-4), 210-219.

Yuan Zx, Lm, W., 2004. Application of high-resolution satellite image for seismic risk assessment. Paper presented at the 13th wrold conference on earthquake engineering, Vancouver, BC, Canada.

Zachos, L. G., Swann, C. T., Altinakar, M. S., Mcgrath, M. Z., Thomas, D., 2016. Flood vulnerability indices and emergency management planning in the Yazoo Basin, Mississippi. International Journal of Disaster Risk Reduction, 18, 89-99.

Zhang, X. R., Wang, Z. B., Lin, J., 2015. GIS Based Measurement and Regulatory Zoning of Urban Ecological Vulnerability. Sustainability, 7(8), 9924-9942. 
Zhao, C., Lu, Z., 2018. Remote Sensing of Landslides-A Review. Remote Sensing, 10(2), 279. 


\section{Chapter 3 - A conceptual framework for post-disaster recovery assessment with remote sensing ${ }^{2}$}

2 This chapter is based on:

- Kerle, N., Ghaffarian, S., Nawrotzki, R., Leppert, G., Lech, M., 2018. Evaluating resilience-centered development interventions with remote sensing. Remote Sens., 11, 2511.

Lech, M., Ghaffarian, S., Kerle, N., Leppert, G., Nawrotzki, R., Moull, K., Harten, S., 2020. A Proof-of-Concept of Integrating Machine Learning, Remote Sensing, and Survey Data in Evaluations. The Measurement of Disaster Resilience in the Philippines. DEval Discussion Paper 1/2020, German Institute for Development Evaluation (DEval), Bonn. 


\section{Abstract}

Natural disasters are projected to increase in number and severity, in part due to climate change, and monitoring and evaluation of the post-disaster recovery process has become crucial to support decision makers and governments after a disaster. Remote sensing as an efficient tool has been used for different components of the disaster risk management (DRM) and recovery assessment, mostly focused on physical assessments. However, a comprehensive recovery assessment includes socio-economic and functional aspects in addition to physical ones, which needs to be approached indirectly using proxies. In this study, we proposed a conceptual framework for post-disaster recovery assessment using remote sensing-based proxies. Then we further improved the framework and linked it to resilience assessment and intervention impact evaluation hypothesizing that resilience-enhancing interventions should result in less damage during a hazard event, or at least quicker recovery. We assessed recovery over a 3 year period of seven municipalities in the central Philippines devastated by Typhoon Haiyan in 2013. We used very high resolution optical images ( $<1$ meter), and created detailed land cover and land use maps for four time points before and after the event, using a machine learning approach with extreme gradient boosting. While a number of causal links between intervention parameters and reconstruction was found, the common notion that a resilient community should recovery better and more quickly could not be confirmed. The study also revealed a number of methodological limitations, such as the high cost for typically commercial image data, the remote sensing analysis likely overestimating damage and thus providing incorrect recovery metrics, and image data catalogues especially for more remote communities often being incomplete. 


\subsection{Introduction}

The average annual global economic damage caused by disasters is difficult to determine, with estimations ranging from about 300 billion US\$ (Mochizuki and Naqvi 2019; UNISDR 2015a) to more than 520 billion US\$ (The World Bank 2017). Predictions of future damages, in particular as part of wider expected losses resulting from climate change, are even harder, with different warming scenarios by the Intergovernmental Panel on Climate Change (IPCC) leading to a wide range of consequences (Kharin et al. 2007), including variable changes in hydro meteorological hazards (Bengtsson et al. 2006). Nevertheless, it is widely accepted that economic consequences will be both severe and spatially highly variable (Hsiang et al. 2017; Tol 2018).

Post-disaster recovery is one of the four main components of the Disaster Risk Management (DRM) cycle. It is the process of reconstructing communities in all their aspects (physical, economic, social, and environmental) to return life, livelihoods, and the built environment to their pre-impact or even better states (Burton et al. 2011). In addition, recovery can serve as the window of opportunity and address the build back better concept.

Post-disaster recovery is the least studied component of the DRM cycle, and there is a need for a conceptual framework for comprehensive post-disaster recovery assessment using new technoligies, i.e. remote sensing (RS). The advantages of RS in terms of synoptic coverage, cost effectiveness, and flexibility in sampling frequency or sensor choice are well recognized in the field of disaster risk management (Kerle 2015b). In the existing literature most of the RS-based methods for recovery assessment focused on the reconstruction part of the recovery, by using change detection techniques to extract whether damaged buildings were reconstructed or new buildings or structures built. In addition, some researchers studied the recovery process in its long-term phase, utilizing indicators such as the reconstruction of bridges and roads for accessibility analysis, change detection in land cover classification to extract environmental changes, and so forth (Brown et al., 2010). Conventionally, recovery was considered as a predictable and orderly process (Haas et al. 1977). However, recent studies demonstrated that the recovery process is more complex (Brown et al. 2015). The complexity of the recovery process is basically because of being a multi-dimensional process; indeed, it needs numerous sectors, stakeholders, policymakers, and so on to take a role and responsibility. Reconstruction and, consequently, recovery starts after the disaster has happened; therefore, governments and disaster planners have to make decisions and act quickly. However, on the one hand, reconstruction of buildings is only the physical part of the recovery, i.e. it omits other important sides, such as economic, social and environmental aspects. There are several socio-economic factors that influence the recovery process 
and its rate from early stage such as social interactions (e.g. social cohesion) (Townshend et al. 2014), or business recovery (Rose and Krausmann 2013). The principal challenge in utilizing RS for recovery assessment is that most image analysis methods developed in this domain focus on the detection or characterization of physical features. Only more recent research has also looked at non-physical properties such as social vulnerability (Ebert et al. 2009) or functional disaster damage. All make use of physical proxy indicators (hereafter: proxies) constituting indirect measures that provide insights regarding not directly observable features or processes (Ghaffarian et al. 2018).

Recently, the Sendai Framework has been defined for disaster risk reduction as a roadmap to make communities safer and resilient to disasters (Unisdr 2015b). It proposes to use the post-disaster as a window of opportunity to build back better, increasing the resilience of the community while reducing its pre-exciting vulnerability. Accordingly, the aim of the post-disaster recovery will be not only to return the community to normal/pre-disaster situation, but also to improve the pre-existing physical, social, economic, and environmental conditions.

Resilience is increasingly becoming a ubiquitous concept in many disciplines, such as economic, sociology, psychology, and healthcare. It has also been considered as an emerging research topic in disaster risk management in recent decades. However, measuring the resiliency of a community has not yet been sufficiently addressed, and it is a challenging topic for DRM researchers. Furthermore, resilience assessment can be used for intervention impact evaluations. It stands to reason that communities that have received several years of DRR interventions should suffer less damage during a hazard event, or at least recover more quickly than unassisted communities. However, assessing the effect of interventions is complicated. This is because of the difficulty (i) to attribute a certain performance or behavior of people or governments to specific interventions, especially when different organizations are active in the area, (ii) to estimate the future effect and value of planning activities or what people learned in a community meeting, and (iii) to collect the very detailed socio-economic data needed to support a quantitative impact evaluation. There has generally been growing interest in transparency and accountability, with auditing agencies but also donors giving post-disaster assistance increasingly requesting evidence of the effect of funded interventions.

Impact evaluations, which can address relevance, effectiveness, efficiency, impact, or sustainability, usually follow a common procedure: first, evaluators collect baseline data prior to the start of the intervention. After the program has been completed and project impacts have crystalized endline data are 
compiled. It is good practice to collect additional performance data during the program implementation stage to carry out adjustments if needed. Data collection is performed in treatment and non-treatment communities. Ideally treatment is assigned randomly (randomized control trial), but if this is not possible, matching methods can be employed (quasi-experimental design) (Gertler et al. 2016).

Traditional impact evaluation methods face a number of limitations: (i) collecting survey data is a time consuming and expensive task (Gertler et al. 2016). Particularly if a program is implemented widely across a country or region, costs can rise drastically and limit either coverage or detail; (ii) traditional survey methods require a long planning horizon and are difficult to implement ad-hoc. This makes it challenging to collect baseline data for programs that respond to sudden onset disasters such as storms, flooding, or earthquakes (Leppert et al. 2018); (iii), traditional survey methods are restricted by access considerations. For example, it is difficult to evaluate programs that take place in very remote locations without proper road access. Similarly, the political situation in a country (e.g., military conflict) may prevent evaluators to collect survey information (Pech 2017).

A prerequisite for employing rigorous evaluation methodologies is sufficient data availability. Traditionally, evaluations have made use of survey methods to collect the necessary data before, during and after an intervention. However, the collection of survey data is expensive and responses may be subjective. In addition, survey data frequently lack the geographical detail if environmental changes are to be evaluated (Lech et al. 2018). The use of RS data to complement traditional evaluation designs offers some major advantages (Nawrotzki 2019), such as archives that often span decades, and thus the availability of image data acquired long before the intervention started. Many satellite products are available free of charge, which may help reduce the costs for data collection. Finally, geographic data can provide an objective measure of high detail for changes in environmental conditions, which is difficult to obtain from survey data alone.

Many methods have been developed to extract information from RS data to measure and characterize different aspects of disaster risk management (Coppola 2015; Kerle 2015b). However, most existing studies have focused on physical assessments using direct observations, be it for hazard or risk assessment, but also to detect post-disaster damage or recovery. For example, researchers have assessed the number of buildings collapsed/damaged (Arciniegas et al. 2007; Saito et al. 2004) and reconstructed (Berke et al. 1993), or estimated their vulnerability (Mueller et al. 2006). However, the nonphysical aspects of DRM, e.g., social and economic properties, are also crucial and should be assessed (MCDEM 2005). However, this requires indirect 
observations and the use of proxies (Ghaffarian et al. 2018), as demonstrated in recent studies on vulnerability (Flax et al. 2002; Morrow 1999), resilience (Burton 2012), damage (Bevington et al. 2010; Bradshow 2004), and recovery (Rubin et al. 1985). In addition, recent studies showed that most of the developed proxies for damage and recovery assessments can be extracted through LC and LU monitoring (Rau et al. 2007; Reif et al. 2011), increasingly through the use of ML techniques (Sheykhmousa et al. 2019). The growing sophistication of image-based extraction of both physical and non-physical indicators of recovery, and thus resilience, suggests a possible role in impact evaluation, which we test in this work.

Our work focused on the effect of interventions on resilience, i.e. the ability to rebound from an adverse event, based on the developed recovery assessment framework with remote sensing. We considered post-disaster recovery to be a proxy for resilience, and thus used RS to detect evidence of spatially variable recovery. Similar to (Sheykhmousa et al. 2019) we assessed land cover (LC) and land use (LU) with machine learning (ML) methods over a 3 year period following a disaster event to detect the level of recovery across space, which was then related to intervention information collected in the field. We developed the proof of concept for a number of barangays (municipalities) in the Leyte region in the central Philippines, which was severely affected during Typhoon Haiyan in 2013.

\subsection{Data and Methods}

\subsubsection{Overall approach}

Figure 3.1 illustrates the conceptual framework for post-disaster recovery assessment and overall methodological approach followed in this study. Using a variety of image-derived proxies that cover the built-up, social, and economic dimensions we first quantified the damage caused by typhoon Haiyan, followed by a quantification of the recovery for different post-event years. The spatially variable recovery performance was considered to reflect the underlying resilience. In the following we first provide details about the study area and the satellite data used. Subsequently we explained the developed proxies, and how they were used in the damage and recovery assessment. Finally the correlation analysis to link the RS results and the impact evaluation data was described. 


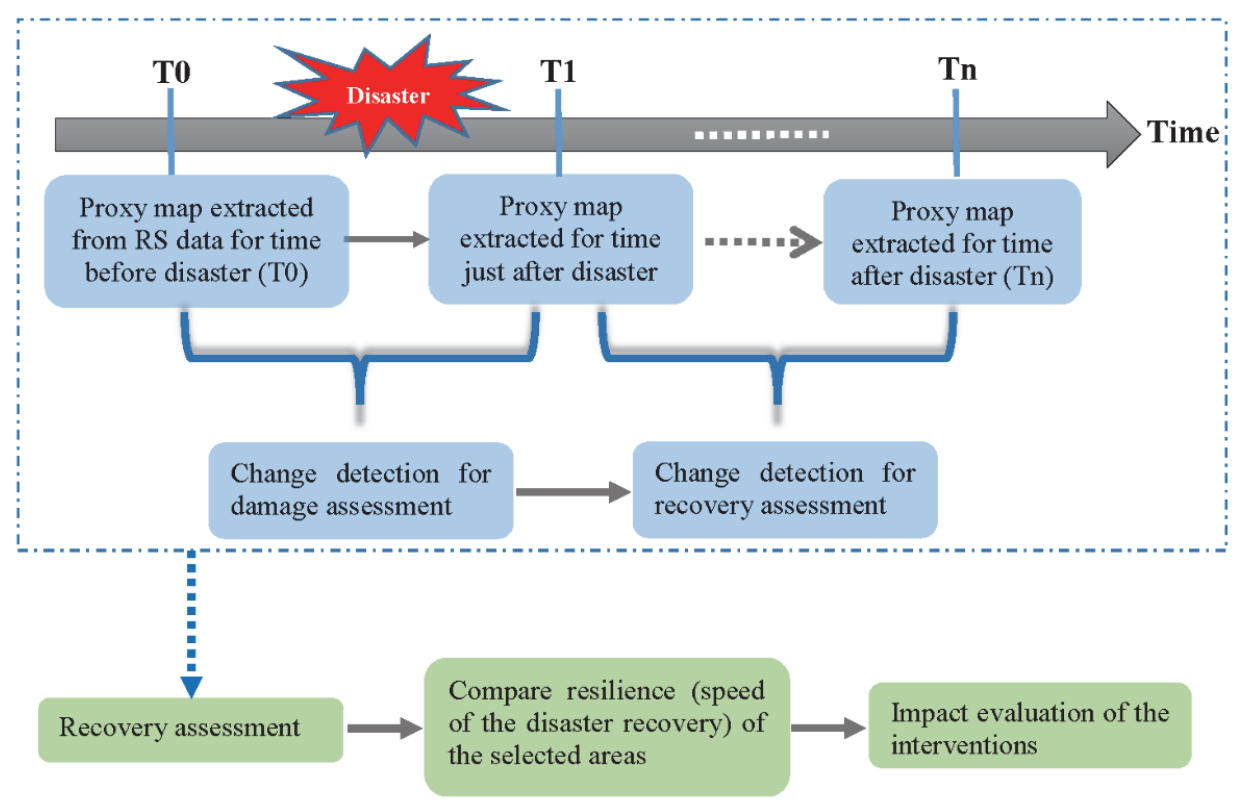

Figure 3.1 The conceptual framework connecting post-disaster recovery assessment using remote sensing-based proxies with resilience assessment and impact evaluation.

\subsubsection{Study location and image data used}

Typhoon Haiyan passed over the Central Visayas region of the Philippines, crossing the islands of Samar and Leyte on 8 November 2013. With sustained wind speeds exceeding $310 \mathrm{~km} / \mathrm{h}$ at landfall, it was one of the strongest tropical storms on record worldwide. The center of the storm passed close to Leyte's largest city and adminstrative center, Tacloban. Reported fatality figures vary widely, though are inofficially estimated to be in excess of 13,000 (Daniell et al. 2013) attributable to strong winds and high waves up to $5 \mathrm{~m}$ along the Basey and Tacloban coastal area (Lagmay et al. 2015). Other sources reported heights of up to $7 \mathrm{~m}$ in Leyte (Takagi and Esteban 2016), and even $12 \mathrm{~m}$ in Samar (Kure et al. 2016). Structural damage was extensive, with more than 1 million buildings getting partially or completely destroyed in nearly 600 affected municipalities. Leyte alone acounted for a reported $86 \%$ of all casualties, and a quarter of the economic losses relate to destroyed rice and corn crops (Boschetti et al. 2015). 


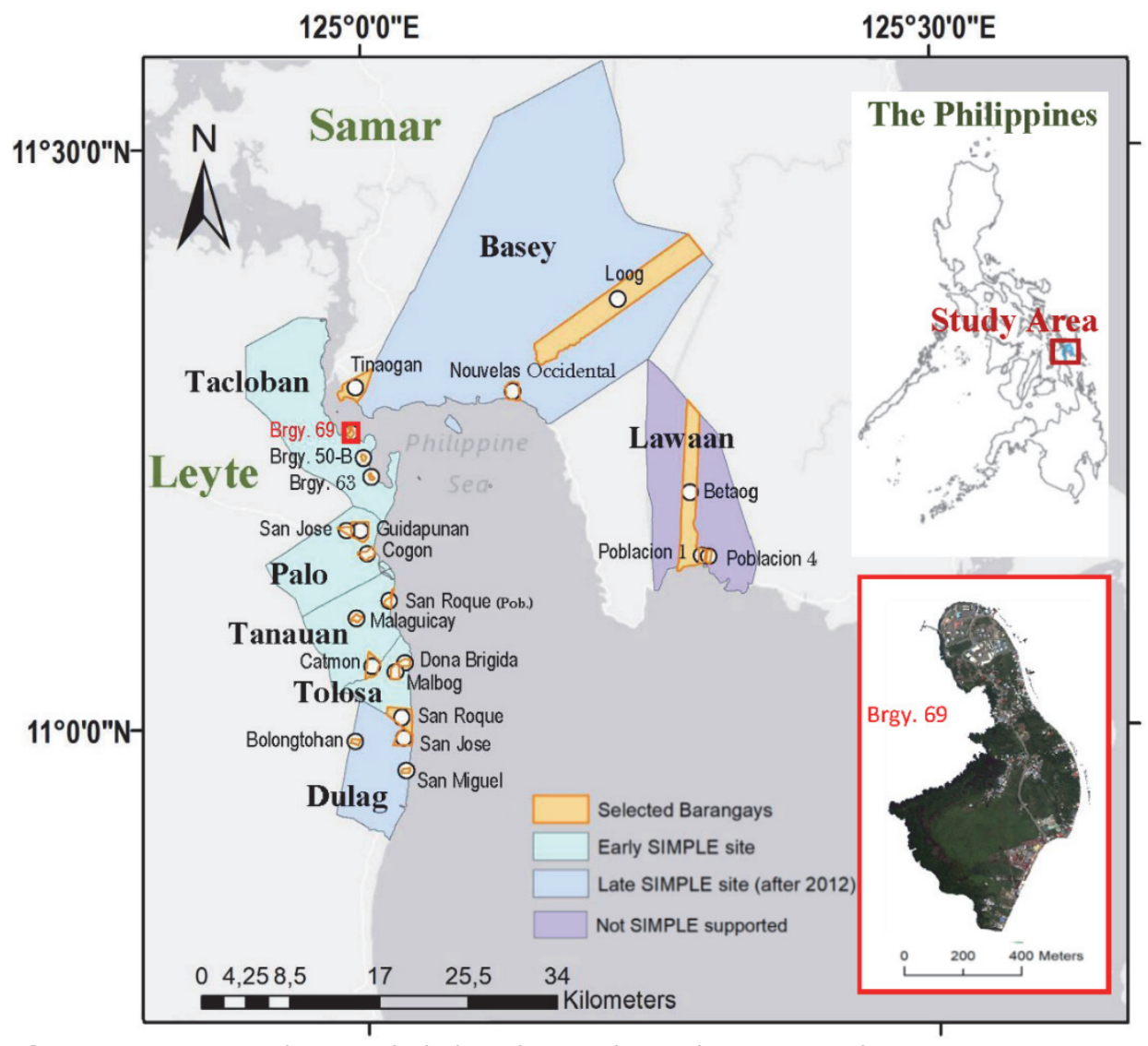

Figure 3.2 Municipalities included in this study, and overview of Barangay 69. Notes: Brgy. = barangay; SIMPLE = Sustainable Integrated Management and Planning for Local Government Ecosystems intervention; GIZ = German agency for technical cooperation.

Very high-resolution satellite images were used to implement the developed LCLU classification approach and to extract relevant proxies, shows the specifications and acquisition times of the images used. 
Table 3.1 Satellite images used in this study.

\begin{tabular}{|c|c|c|c|c|}
\hline Area & Satellite & Acquisition date & $\begin{array}{c}\text { Time }[T 0, \\
T 1, T 2, T 3]^{1}\end{array}$ & $\begin{array}{c}\text { Spatial } \\
\text { resolution }^{2} \\
{[\mathrm{MS} ; \mathrm{Pan}]}\end{array}$ \\
\hline Tacloban & WorldView2 & 2013-03-17 & T0 & $2 \mathrm{~m} ; 0.5 \mathrm{~m}$ \\
\hline Tacloban & WorldView2 & 2017-03-18 & $\mathrm{T} 3$ & $2 \mathrm{~m} ; 0.5 \mathrm{~m}$ \\
\hline Tacloban & GeoEye1 & 2013-11-10 & $\mathrm{T} 1$ & $2 \mathrm{~m} ; 0.5 \mathrm{~m}$ \\
\hline Tacloban & GeoEye1 & 2013-11-12 & $\mathrm{T} 1$ & $2 \mathrm{~m} ; 0.5 \mathrm{~m}$ \\
\hline Tacloban & GeoEye1 & 2013-11-13 & $\mathrm{T} 1$ & $2 \mathrm{~m} ; 0.5 \mathrm{~m}$ \\
\hline Tacloban & GeoEye1 & $2016-04-24$ & $\mathrm{~T} 2$ & $2 \mathrm{~m} ; 0.5 \mathrm{~m}$ \\
\hline South Leyte & WorldView2 & 2013-03-25 & T0 & $2 \mathrm{~m} ; 0.5 \mathrm{~m}$ \\
\hline South Leyte & WorldView2 & 2013-04-02 & T0 & $2 \mathrm{~m} ; 0.5 \mathrm{~m}$ \\
\hline South Leyte & WorldView2 & 2014-01-07 & $\mathrm{T} 1$ & $2 \mathrm{~m} ; 0.5 \mathrm{~m}$ \\
\hline South Leyte & WorldView2 & $2014-07-16$ & $\mathrm{~T} 2$ & $2 \mathrm{~m} ; 0.5 \mathrm{~m}$ \\
\hline South Leyte & WorldView2 & 2014-09-11 & $\mathrm{T} 2$ & $2 \mathrm{~m} ; 0.5 \mathrm{~m}$ \\
\hline South Leyte & WorldView2 & 2014-10-21 & $\mathrm{T} 2$ & $2 \mathrm{~m} ; 0.5 \mathrm{~m}$ \\
\hline South Leyte & WorldView2 & 2014-12-01 & $\mathrm{T} 2$ & $2 \mathrm{~m} ; 0.5 \mathrm{~m}$ \\
\hline South Leyte & WorldView2 & 2016-01-24 & $\mathrm{T} 3$ & $2 \mathrm{~m} ; 0.5 \mathrm{~m}$ \\
\hline South Leyte & WorldView2 & $2016-06-24$ & $\mathrm{~T} 3$ & $2 \mathrm{~m} ; 0.5 \mathrm{~m}$ \\
\hline Basey & WorldView2 & 2013-05-18 & T0 & $2 \mathrm{~m} ; 0.5 \mathrm{~m}$ \\
\hline Basey & WorldView2 & 2013-05-18 & T0 & $2 \mathrm{~m} ; 0.5 \mathrm{~m}$ \\
\hline Basey & WorldView2 & 2013-09-01 & T0 & $2 \mathrm{~m} ; 0.5 \mathrm{~m}$ \\
\hline Basey & WorldView2 & 2013-11-19 & $\mathrm{T} 1$ & $2 \mathrm{~m} ; 0.5 \mathrm{~m}$ \\
\hline Basey & WorldView2 & 2013-11-21 & $\mathrm{T} 1$ & $2 \mathrm{~m} ; 0.5 \mathrm{~m}$ \\
\hline Basey & WorldView3 & $2014-12-09$ & $\mathrm{~T} 2$ & $1.3 \mathrm{~m} ; 0.31 \mathrm{~m}$ \\
\hline Basey & WorldView3 & 2015-01-10 & $\mathrm{T} 2$ & $1.3 \mathrm{~m} ; 0.31 \mathrm{~m}$ \\
\hline Basey & WorldView2 & 2016-06-04 & $\mathrm{T} 3$ & $2 \mathrm{~m} ; 0.5 \mathrm{~m}$ \\
\hline Basey & WorldView2 & 2016-06-04 & $\mathrm{T} 3$ & $2 \mathrm{~m} ; 0.5 \mathrm{~m}$ \\
\hline Lawaan & WorldView2 & 2013-05-18 & T0 & $2 \mathrm{~m} ; 0.5 \mathrm{~m}$ \\
\hline Lawaan & WorldView2 & 2013-05-18 & T0 & $2 \mathrm{~m} ; 0.5 \mathrm{~m}$ \\
\hline Lawaan & WorldView2 & 2014-01-07 & $\mathrm{T} 1$ & $2 \mathrm{~m} ; 0.5 \mathrm{~m}$ \\
\hline Lawaan & WorldView2 & 2014-01-07 & $\mathrm{T} 1$ & $2 \mathrm{~m} ; 0.5 \mathrm{~m}$ \\
\hline Lawaan & WorldView3 & 2014-10-07 & $\mathrm{T} 2$ & $1.3 \mathrm{~m} ; 0.31 \mathrm{~m}$ \\
\hline Lawaan & WorldView2 & 2015-11-24 & $\mathrm{T} 3$ & $2 \mathrm{~m} ; 0.5 \mathrm{~m}$ \\
\hline
\end{tabular}

T0 - pre-disaster, T1 - shortly after Haiyan, T2 and T3 - approx. 2 years and 3 years after the disaster, respectively. 2 MS - multispectral, Pan - panchromatic

\subsubsection{Remote sensing data analysis}

We developed a conceptual framework to assess recovery based on the extraction of image-based proxies (Figure 3.1). The approach consisted of two main steps: (i) to generate the damage map, and (ii) to generate the recovery map at the required times after the disaster. To do so, suitable proxies needed to be identified and extracted for each of the required time steps (e.g., predisaster, event time and post-disaster). Proxies for separate dimensions, i.e. built-up, social, and economic (Ghaffarian et al. 2018) are needed to cover the entire damage and recovery process comprehensively. The proxies listed for the pre-disaster (TO) and event (T1) times support the damage assessment, and subsequently the damage map was compared with the proxies listed for two post-disaster time epochs approximately 2 and 3 years after Haiyan (T2 
and T3) to assess recovery at each time. The changes in the state of each proxy between two epochs demonstrate the damage to the indicators, and subsequently the degree of recovery.

Table 3.2 shows the selected proxies and their use to evaluate recovery in the selected study area. To assess the full scope of damages we employed proxies that measure the structural damages to buildings, bridges and transportation facilities, but also textural proxies and evidence of blow-out debris. To assess the recovery process we employ proxies that measure the reconstruction of buildings and impervious surface. Once the proxy-based damage map has been produced, monitoring changes through the built-up proxies indicates the degree of recovery at a given point in time following the disaster. Among the economic proxies, LU information indicates the types of economic activity, while the presence of vehicles and boats provides an indication of the extent and frequency of its use. Arable land is a proxy for the potential for farming. In addition, roof color and material helps to differentiate between types of buildings (e.g., industrial facilities vs. residential housing). We use the share of population residing in informal settlements (i.e., slums) as a proxy for the socio-economic status of the area.

Machine learning methods such as support vector machines (SVM) and random forest (RF) have been widely used in remote sensing data processing, particularly for satellite image processing, due to their efficiency and accurate results. Several researchers also studied their applicability in producing accurate LC and LU maps from remote sensing optical images (Georganos et al. 2018a; Ma et al. 2017; Maxwell et al. 2018; Nery et al. 2016; Sheykhmousa et al. 2019). Recent advances in computer science motivated researchers to use deep learning and convolutional neural network (CNN) approaches as superior classification methods (Yang et al. 2018; Zhu and Newsam 2015). However, CNN-based models must be trained with a large number of samples to give appropriate results, which also requires substantially more computational power and complex models.

Other advanced ML methods have been developed to support challenging image classification tasks at low computational costs, such as logistic model trees, and rotation forest ensembles. Gradient boosting method (GBM) is a supervised classification technique and belongs to regression and classification trees models (Friedman 2001). Tree boosting is an ensemble learning algorithm that is very effective in the classification of even weak trees (Ren et al. 2017), as has been shown in scene classification (Chan and Paelinckx 2008; Zhang et al. 2016). However, traditional GBMs require the tuning of a number of parameters and are thus more susceptible to overfitting than other ML algorithms, such as SVM. In 2008, Chen and Guestrin (Chen and Guestrin 2016) developed the Extreme Gradient Boosting (XGBoost) method, which is 
the regularized version of GBM and overcomes most of the limitations. Since then XGBoost has been successfully used for different classification problems.

Table 3.2. The proxies used for the recovery assessment.

\begin{tabular}{|c|c|c|}
\hline Proxy & $\begin{array}{l}\text { Categor } \\
y\end{array}$ & Description \\
\hline Building damage & Built up & Number of damaged/ collapsed buildings \\
\hline Texture & Built up & $\begin{array}{l}\text { To extract damaged buildings, roads, } \\
\text { and urban areas }\end{array}$ \\
\hline $\begin{array}{l}\text { Building removal and } \\
\text { reconstruction }\end{array}$ & Built up & To detect recovered buildings \\
\hline Blow-out debris & Built up & $\begin{array}{l}\text { To detect blocked roads and damaged } \\
\text { buildings }\end{array}$ \\
\hline $\begin{array}{l}\text { Reconstruction of bridges } \\
\text { and public transport facilities }\end{array}$ & Built up & $\begin{array}{l}\text { Accessibility and transportation facility } \\
\text { assessments }\end{array}$ \\
\hline Impervious surface & Built up & $\begin{array}{l}\text { To extract built-up area and permeability } \\
\text { of the surface }\end{array}$ \\
\hline Presence of vehicles & $\begin{array}{l}\text { Built up/ } \\
\text { Economic }\end{array}$ & $\begin{array}{l}\text { For transportation condition/ } \\
\text { accessibility analysis and functioning of } \\
\text { roads, and extract level of economic } \\
\text { activities }\end{array}$ \\
\hline $\begin{array}{l}\text { Land use (large-scale } \\
\text { industry) }\end{array}$ & Economic & $\begin{array}{l}\text { Economic activity types/ location and } \\
\text { economic focal spaces }\end{array}$ \\
\hline Presence of boats & Economic & Fishery industry for livelihood recovery \\
\hline Arable land & Economic & $\begin{array}{l}\text { Agriculture industry for livelihood } \\
\text { recovery }\end{array}$ \\
\hline Roof color and material & Economic & Industry recovery \\
\hline $\begin{array}{l}\text { Proportion of built-up and } \\
\text { vegetated area }\end{array}$ & Social & Settlement type/ location in urban areas \\
\hline $\begin{array}{l}\text { Share of population in } \\
\text { irregular clusters }\end{array}$ & Social & $\begin{array}{l}\text { Irregular clusters as roof types refer to } \\
\text { people with low income/ economy (slum } \\
\text { area) }\end{array}$ \\
\hline
\end{tabular}

Its superiority for LC and LU classification from very high resolution images was also shown in recent studies (Georganos et al. 2018b; Ren et al. 2017). Both urban and rural LU classes in most cases cannot be distinguished using only spectral information (e.g., slum areas), and require the addition of spatial features (i.e., texture) (Chen et al. 2015). Local Binary Patterns have been shown to be an effective textural information extraction method (Kuffer et al. 2016; Mboga et al. 2017; Sheykhmousa et al. 2019). LBP are based on the gray level co-occurrence matrix, which contains simple textural computations such as mean, variance, homogeneity and entropy. Therefore, in this study the LBP of each image, using a $5 \times 5$ kernel, were computed and used as input in addition to the image bands in the XGBoost classification. The implementation of the XGBoost classifier was based on the default parameter values, and the calculation of the LBP and XGBoost was performed in Python. 
The LC map contains the following classes: building, impervious surface, bare land, inland water, trees/flattened trees, non-tree vegetation, and rubble for the event time images. In addition, the LU maps include large-scale industry, informal settlement (slums), formal buildings, trees/flattened trees, crop land, grass land, inland water, bare land, impervious surface, and rubble for event time images. In the first step of the classification the training areas were selected for each class, separately for the LC and LU classifications. In addition, the accuracy of the results was computed based on standard measurements, overall, user, and producer accuracy (OA, UA, and PA, respectively) for Barangay 69. Stratified random sampling was used to generate random reference points for the evaluation. Considering the size of the test area, a minimum of 150 sample pixels for both the LC and LU classification maps was selected to produce reference data.

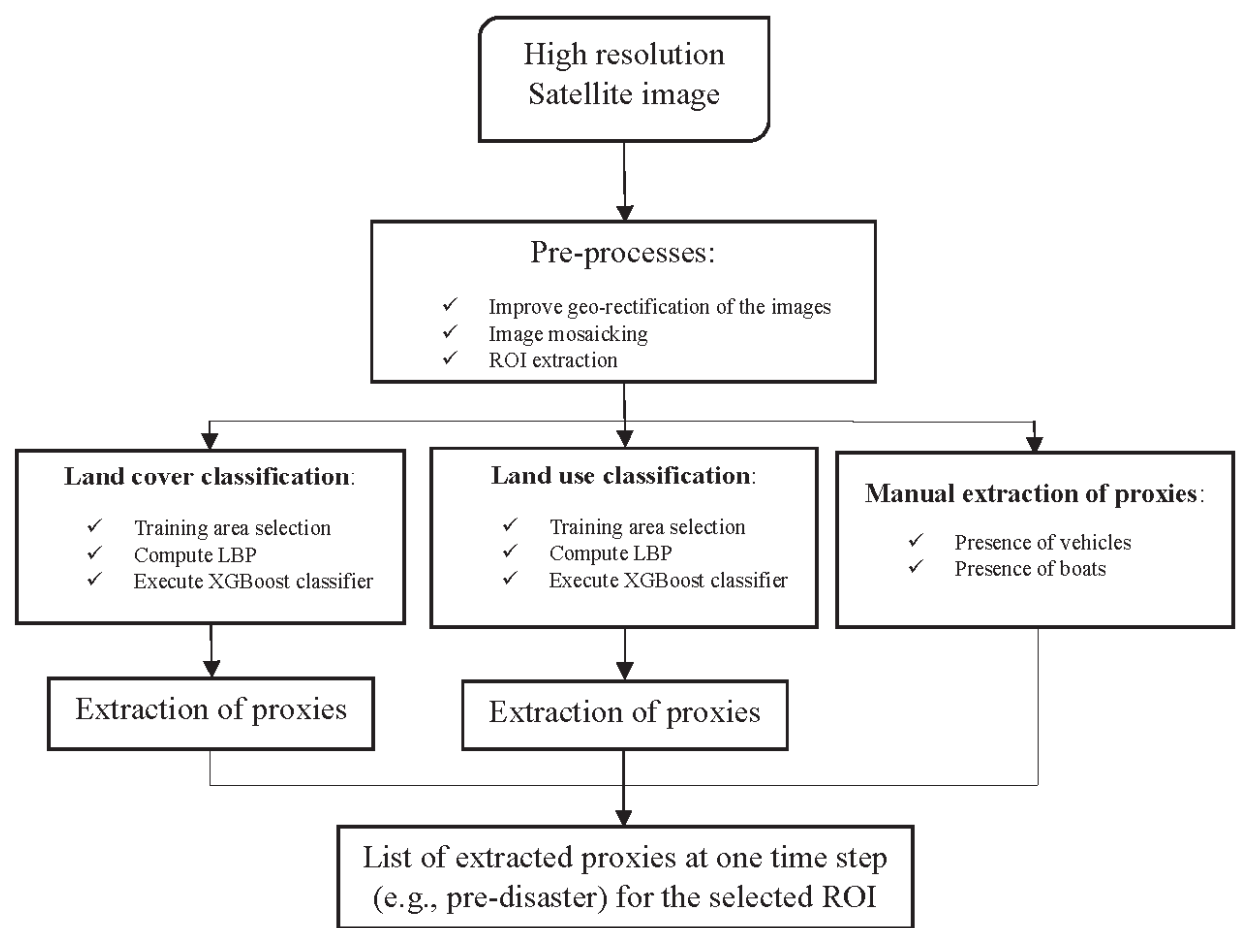

Figure 3.3. Framework for the extraction of proxies using high resolution satellite images for each region of interest (ROI) at each time step (e.g., pre-disaster, T0).

Figure 3.3.3 shows the general framework of the implemented steps for the proxy extraction based on LCLU maps. Before starting to select the training areas for the classification, three pre-processes were implemented: (i) improving the geo-referencing of the images; since the final recovery map/proxy extraction was conducted based on a pixel-by-pixel comparison of the maps for each time step, the images required a good matching in terms of 
geo-referencing. Accordingly, one of the images was selected as a base image, and the geo-referencing of the other images refined using image-to-image registration/rectification, as is commonly done in pixel-based change detection techniques (Singh 1989). However, satellite inclination angles vary, and in particular the time-critical post-disaster response phase tends to be dominated by images taken at large off-nadir angles. This leads to errors in the change assessment, in particular for vertical features such as building façades; (ii) Image mosaicking to match and merge different satellite images to cover the entire area. The merging operation, and thus the provided merged images, challenge the classification approach and lead to inaccuracies in the LCLU maps, particularly for images collected in different seasons or with large gaps between acquisitions; (iii) ROI/barangay image extraction, which was conducted using ArcGIS based on the barangay boundaries by the United Nation's Office for Humanitarian Affairs (OCHA) based on information provided by the Philippine Statistical Authority (PSA) (The Humanitarian Data Exchange 2019).

The computed class characteristics were used for the proxy extraction and interpretations. The LCLU classification was implemented on multi-spectral satellite images, while due to the small size of the vehicles and boats compared to other LCLU classes, they were extracted using both panchromatic and multispectral images. The boats inside the barangays (on inland water), as well as the boats close to the selected barangays (on open water) were counted. The proxies were extracted based on the LCLU class area sizes and their changes from (TO-T3) except number of boats and vehicles, which were manually counted. For example, the building land cover change from the predisaster time (TO) to just after disaster time (T1) shows the change in the overall size of the buildings in the area and consequently the damaged buildings. Furthermore, roof color was calculated based on the extraction of the brightness values of the pixels for the building class using MATLAB.

We implemented the developed approach on the images of the 4 epochs considered (T0-T3) to extract the selected proxies.

\subsection{Results}

The main analysis was conducted based on the proposed methodology for the selected barangays from each municipality. Barangay $69\left(0.38 \mathrm{~km}^{2}\right.$ in size), located in the North of Tacloban (Figure 3.2), was selected to visualize the analysis and explain the results in detail for each step. This barangay contains most of the LCLU classes, in addition to some representative challenges/inaccuracies we encountered during the classification and providing the final recovery assessment results. 
Figure 3.4 and Figure 3.5 show the LC and LU results and their corresponding area coverage ratios for the entire Barangay 69 , respectively. The original true color images as well as LCLU maps are provided for each time step to visualize the recovery. However, the selection of the training areas was challenging for some classes, particularly for LU, such as distinguishing the large-scale industry facilities from formal buildings without using auxiliary data.

The developed approach resulted in overall accuracy scores of $85.4 \%, 76.1 \%$, $83.4 \%$ and $86.0 \%$ for the LC classification at T0, T1, T2 and T3 images, respectively, which demonstrate the robustness of the method in classifying the land covers from satellite images (Table 3.3). However, the user accuracy (UA) values of the Impervious surface and Water classes are, $66.7 \%$ and $60.0 \%$, respectively that show the relatively high commission error level in those classes. In addition, overall accuracies of the LU maps produced by the developed method are $81.8 \%, 72.1 \%, 78.7 \%$ and $78.8 \%$ for T0, T1, T2 and T3 images, respectively (As can be seen from Figure 3.5 most of the buildings recovered, while half of the trees had not yet recovered by T3. The latter was to be expected, given that an estimated 33 million coconut trees were destroyed by Haiyan in the Visayas (Acda 2015), which take 6-8 years to regrow to maturity. This also explains an observed shift in cultivation of palm trees to other crop types, which leads to a decrease in the number of trees in the area. In addition, young replanted trees are not yet detectable in satellite images, which results in them being misclassified as grass or crop land.

Shows the final extracted proxies. Almost $80 \%$ of the buildings in Barangay 69 had been reconstructed/ had recovered by March 2017. However, the area covered by both industrial buildings and informal settlements (slums) decreased. The latter are marked by clusters with highly irregular patterns, and their share decreased, which illustrates a positive recovery aspect after the disaster.

Table 3.4). More classes and their similarity in the LU classification, which challenges the classification process, resulted in lower accuracy scores when compared to the LC classification. However, the high producer accuracy (PA) values for most of the land use classes ranging from $70 \%$ to $100 \%$ demonstrates the low level of omission errors for those classes in such a challenging case. The developed method produced accurate results in classifying roads (impervious surface) and built up areas, as well as the vegetation classes. However, some inaccuracies resulted in particular from the presence of clouds and their corresponding shadows. For example, the T1 image contains a shadowed area that is mainly covered with low-level 
vegetation and trees; however, in the LCLU maps the area is classified as debris/rubble (Figure 3.4, Figure 3.5).

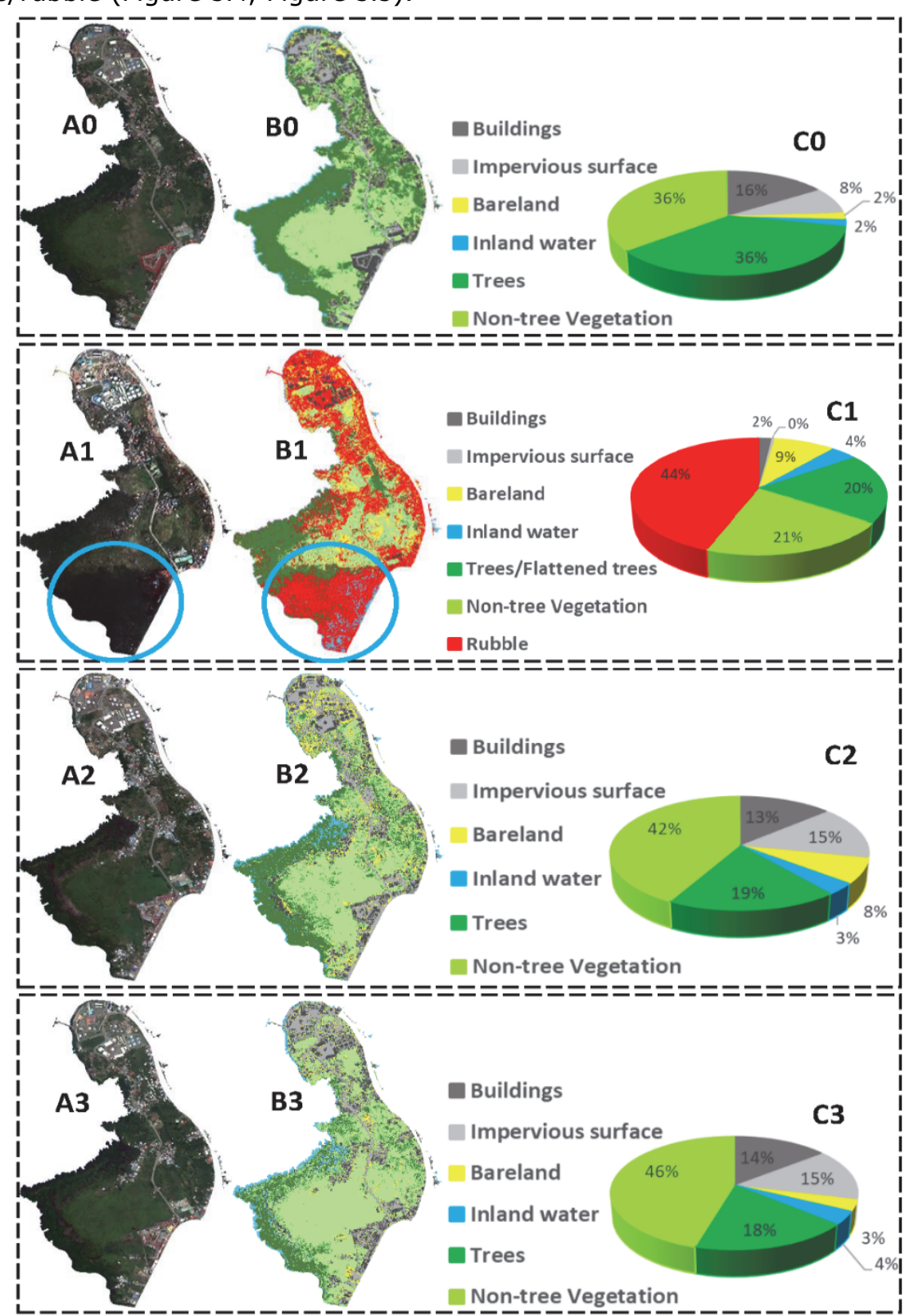

Figure 3.4 $A 0, A 1, A 2, A 3$ : Original very high resolution WorldView2, GeoEye1, GeoEye1, and WorldView2 satellite images, respectively, acquired over Barangay 69, Tacloban city from 8 months before (T0), right after (T1), 2 years (T3) and 4 years after Typhoon Haiyan (T3), respectively; B0,B1,B2,B3: LC classification result for the four time epochs; $\mathrm{C} 0, \mathrm{C} 1, \mathrm{C} 2, \mathrm{C} 3$ : corrresponding pie charts show ditribution of the $\mathrm{LC}$ classes. The area denoted by the blue circles in A1 and B1 shows the shadowed area in the image. 


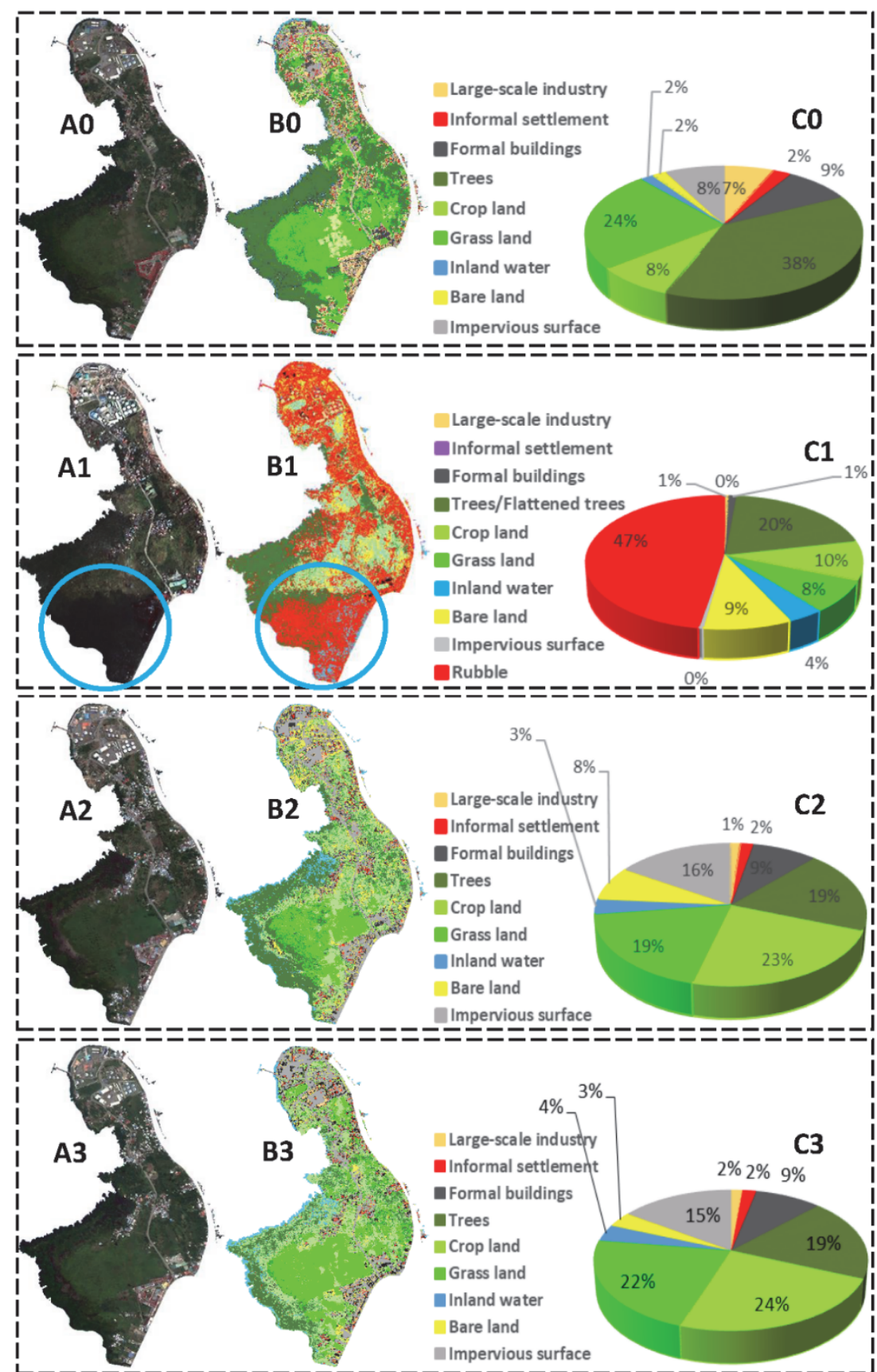

Figure 3.5 $A 0, A 1, A 2, A 3$ : Original very high resolution WorldView2, GeoEye1, GeoEye1, and WorldView2 satellite images, respectively, acquired over Barangay 69, Tacloban city, 8 months before (T0), right after (T1), 2 years (T3) and 4 years after Typhoon Haiyan (T3), respectively; $\mathrm{B} 0, \mathrm{~B} 1, \mathrm{~B} 2, \mathrm{~B} 3$ : LU classification result for the four time epochs; $\mathrm{C} 0, \mathrm{C} 1, \mathrm{C} 2, \mathrm{C} 3$ : corrresponding pie charts show ditribution of the LU classes. The area denoted by the blue circles in $\mathrm{A} 1$ and $\mathrm{B} 1$ shows the shadowed area in the image. 
Table 3.3 The LC classification accuracies for T0, T1, T2 and T3 time epochs for Barangay 69. User and producer accuracies and corresponding errors are computed across the study area from the confusion matrices. PA - Producer's Accuracy; UA - User's Accuracy; OA - Overall Accuracy; N.S - Number of Smaples used for accruacy assessment.

\begin{tabular}{|c|c|c|c|c|c|c|c|c|}
\hline \multicolumn{2}{|c|}{ Time/Class } & $\begin{array}{l}\text { Building } \\
\mathbf{s}\end{array}$ & $\begin{array}{l}\text { Imperviou } \\
\text { s Surface }\end{array}$ & $\begin{array}{c}\text { Bar } \\
\text { e } \\
\text { land }\end{array}$ & $\begin{array}{c}\text { Wate } \\
\mathbf{r}\end{array}$ & $\begin{array}{c}\text { Tree } \\
\mathbf{s}\end{array}$ & $\begin{array}{c}\text { Non-tree } \\
\text { vegetati } \\
\text { on }\end{array}$ & Rubble \\
\hline \multirow{4}{*}{$\begin{array}{l}\text { Pre- } \\
\text { disaster } \\
\text { (T0) }\end{array}$} & N.S. & 40 & 40 & 40 & 40 & 40 & 40 & --- \\
\hline & $\begin{array}{l}\text { PA } \\
\%\end{array}$ & 72.0 & 89.2 & 82.9 & 95.0 & 97.1 & 81.8 & --- \\
\hline & $\begin{array}{l}\text { UA } \\
\%\end{array}$ & 90.0 & 82.5 & 72.5 & 95.0 & 82.5 & 90.0 & --- \\
\hline & $\begin{array}{l}\text { OA } \\
\%\end{array}$ & \multicolumn{7}{|c|}{85.4} \\
\hline \multirow{4}{*}{$\begin{array}{l}\text { Just } \\
\text { after } \\
\text { disaster } \\
\text { (T1) }\end{array}$} & N.S. & 40 & 30 & 30 & 30 & 30 & 30 & 44 \\
\hline & $\begin{array}{l}\text { PA } \\
\%\end{array}$ & 92.5 & 76.9 & 80.0 & 94.7 & 65.9 & 79.4 & 58.7 \\
\hline & $\begin{array}{l}\text { UA } \\
\%\end{array}$ & 92.5 & 66.7 & 66.7 & 60.0 & 96.7 & 90.0 & 61.1 \\
\hline & $\begin{array}{l}\text { OA } \\
\%\end{array}$ & \multicolumn{7}{|c|}{76.1} \\
\hline \multirow{4}{*}{$\begin{array}{l}\text { Post- } \\
\text { disaster } \\
\text { (T2) }\end{array}$} & N.S. & 30 & 30 & 30 & 30 & 30 & 37 & --- \\
\hline & $\begin{array}{l}\text { PA } \\
\%\end{array}$ & 69.2 & 100 & 82.8 & 96.2 & 72.2 & 91.7 & --- \\
\hline & $\begin{array}{l}\text { UA } \\
\%\end{array}$ & 90.0 & 70.0 & 80.0 & 83.3 & 86.7 & 89.2 & --- \\
\hline & $\begin{array}{l}\text { OA } \\
\%\end{array}$ & \multicolumn{7}{|c|}{83.4} \\
\hline \multirow{4}{*}{$\begin{array}{l}\text { Post- } \\
\text { disaster } \\
\text { (T3) }\end{array}$} & N.S. & 30 & 30 & 30 & 30 & 30 & 50 & --- \\
\hline & $\begin{array}{l}\text { PA } \\
\%\end{array}$ & 71.1 & 87.5 & 81.5 & 100 & 85.3 & 93.5 & --- \\
\hline & $\begin{array}{l}\text { UA } \\
\%\end{array}$ & 90.0 & 70.0 & 73.3 & 100 & 96.7 & 86.0 & --- \\
\hline & $\begin{array}{l}\text { OA } \\
\%\end{array}$ & \multicolumn{7}{|c|}{86.0} \\
\hline
\end{tabular}

As can be seen from Figure 3.5 most of the buildings recovered, while half of the trees had not yet recovered by T3. The latter was to be expected, given that an estimated 33 million coconut trees were destroyed by Haiyan in the Visayas (Acda 2015), which take 6-8 years to regrow to maturity. This also explains an observed shift in cultivation of palm trees to other crop types, which leads to a decrease in the number of trees in the area. In addition, young replanted trees are not yet detectable in satellite images, which results in them being misclassified as grass or crop land.

Shows the final extracted proxies. Almost $80 \%$ of the buildings in Barangay 69 had been reconstructed/ had recovered by March 2017. However, the area covered by both industrial buildings and informal settlements (slums) decreased. The latter are marked by clusters with highly irregular patterns, and their share decreased, which illustrates a positive recovery aspect after the disaster. 
Table 3.4 The LU classification accuracies for T0, T1, T2 and T3 for Barangay 69. User and producer accuracies and corresponding errors were computed across the study area from the confusion matrices. BareL: Bare land; FB: Formal built-up area; ImS: Impervious surface; LSI: Large scale industry; IS: Informal settlement; PA - Producer's Accuracy; UA - User's Accuracy; OA - Overall Accuracy; N.S. - Number of Smaples used for accruacy assessment.

\begin{tabular}{|c|c|c|c|c|c|c|c|c|c|c|c|}
\hline \multicolumn{2}{|c|}{ Time/Class } & $\begin{array}{c}\text { Bare } \\
\text { L }\end{array}$ & $\begin{array}{c}\text { Cro } \\
\text { p }\end{array}$ & FB & $\begin{array}{c}\text { Gras } \\
\mathbf{s}\end{array}$ & $\begin{array}{c}\text { Im } \\
S\end{array}$ & $\begin{array}{c}\text { Wate } \\
\mathbf{r}\end{array}$ & LSI & IS & $\begin{array}{c}\text { Tree } \\
\mathbf{s}\end{array}$ & $\begin{array}{c}\text { Rubbl } \\
\text { e }\end{array}$ \\
\hline \multirow{4}{*}{$\begin{array}{c}\text { Pre- } \\
\text { disaster } \\
\text { (TO) }\end{array}$} & N.S & 30 & 30 & 35 & 30 & 30 & 30 & 30 & 30 & 40 & --- \\
\hline & $\begin{array}{l}\text { PA } \\
\%\end{array}$ & 78.6 & 95.7 & $\begin{array}{c}75 . \\
9\end{array}$ & 90.3 & $\begin{array}{c}71 . \\
0\end{array}$ & 78.8 & $\begin{array}{c}87 . \\
5\end{array}$ & $\begin{array}{c}53 . \\
5\end{array}$ & 90.7 & --- \\
\hline & $\begin{array}{l}\text { UA } \\
\%\end{array}$ & 73.3 & 73.3 & $\begin{array}{c}73 . \\
3\end{array}$ & 86.7 & $\begin{array}{c}73 . \\
3\end{array}$ & 86.7 & $\begin{array}{c}70 . \\
0\end{array}$ & $\begin{array}{c}76 . \\
7\end{array}$ & 97.5 & --- \\
\hline & $\begin{array}{l}\text { OA } \\
\%\end{array}$ & \multicolumn{10}{|c|}{81.8} \\
\hline \multirow{4}{*}{$\begin{array}{c}\text { Just } \\
\text { after } \\
\text { disaster } \\
\text { (T1) }\end{array}$} & $\begin{array}{c}\text { N.S } \\
.\end{array}$ & 30 & 30 & 30 & 30 & 30 & 30 & 36 & 30 & 30 & 47 \\
\hline & $\begin{array}{l}\text { PA } \\
\%\end{array}$ & 74.2 & 100 & $\begin{array}{c}78 . \\
3\end{array}$ & 60.5 & $\begin{array}{c}72 . \\
4\end{array}$ & 100 & $\begin{array}{c}71 . \\
1\end{array}$ & --- & 55.8 & 61.4 \\
\hline & $\begin{array}{l}\text { UA } \\
\%\end{array}$ & 76.7 & 53.3 & $\begin{array}{c}60 . \\
0\end{array}$ & 76.7 & $\begin{array}{c}70 . \\
0\end{array}$ & 50.0 & $\begin{array}{c}88 . \\
9\end{array}$ & --- & 96.7 & 67.5 \\
\hline & $\begin{array}{l}\text { OA } \\
\%\end{array}$ & \multicolumn{10}{|c|}{72.1} \\
\hline \multirow{4}{*}{$\begin{array}{l}\text { Post- } \\
\text { disaster } \\
\text { (T2) }\end{array}$} & N.S & 30 & 30 & 32 & 30 & 30 & 30 & 30 & 30 & 31 & --- \\
\hline & $\begin{array}{l}\text { PA } \\
\%\end{array}$ & 87.1 & 93.8 & $\begin{array}{c}75 . \\
9\end{array}$ & 64.1 & $\begin{array}{c}91 . \\
3\end{array}$ & 96.3 & $\begin{array}{c}70 . \\
0\end{array}$ & $\begin{array}{c}60 . \\
6\end{array}$ & 66.7 & --- \\
\hline & $\begin{array}{l}\text { UA } \\
\%\end{array}$ & 90.0 & 50.0 & $\begin{array}{c}73 . \\
3\end{array}$ & 83.3 & $\begin{array}{c}70 . \\
0\end{array}$ & 86.7 & $\begin{array}{c}70 . \\
0\end{array}$ & $\begin{array}{c}66 . \\
7\end{array}$ & 75.0 & --- \\
\hline & $\begin{array}{l}\text { OA } \\
\%\end{array}$ & \multicolumn{10}{|c|}{78.7} \\
\hline \multirow{4}{*}{$\begin{array}{c}\text { Post- } \\
\text { disaster } \\
\text { (T3) }\end{array}$} & $\begin{array}{c}\text { N.S } \\
.\end{array}$ & 30 & 30 & 30 & 30 & 30 & 30 & 30 & 30 & 30 & --- \\
\hline & $\begin{array}{l}\text { PA } \\
\%\end{array}$ & 90.9 & 100 & $\begin{array}{c}67 . \\
7\end{array}$ & 80.0 & $\begin{array}{c}80 . \\
0\end{array}$ & 90.3 & $\begin{array}{c}61 . \\
1\end{array}$ & $\begin{array}{c}63 . \\
6\end{array}$ & 70.7 & --- \\
\hline & $\begin{array}{l}\text { UA } \\
\%\end{array}$ & 66.7 & 53.3 & $\begin{array}{c}70 . \\
0\end{array}$ & 93.3 & $\begin{array}{c}66 . \\
7\end{array}$ & 93.3 & $\begin{array}{c}73 . \\
3\end{array}$ & $\begin{array}{c}70 . \\
0\end{array}$ & 72.5 & --- \\
\hline & $\begin{array}{l}\text { OA } \\
\%\end{array}$ & \multicolumn{10}{|c|}{78.8} \\
\hline
\end{tabular}




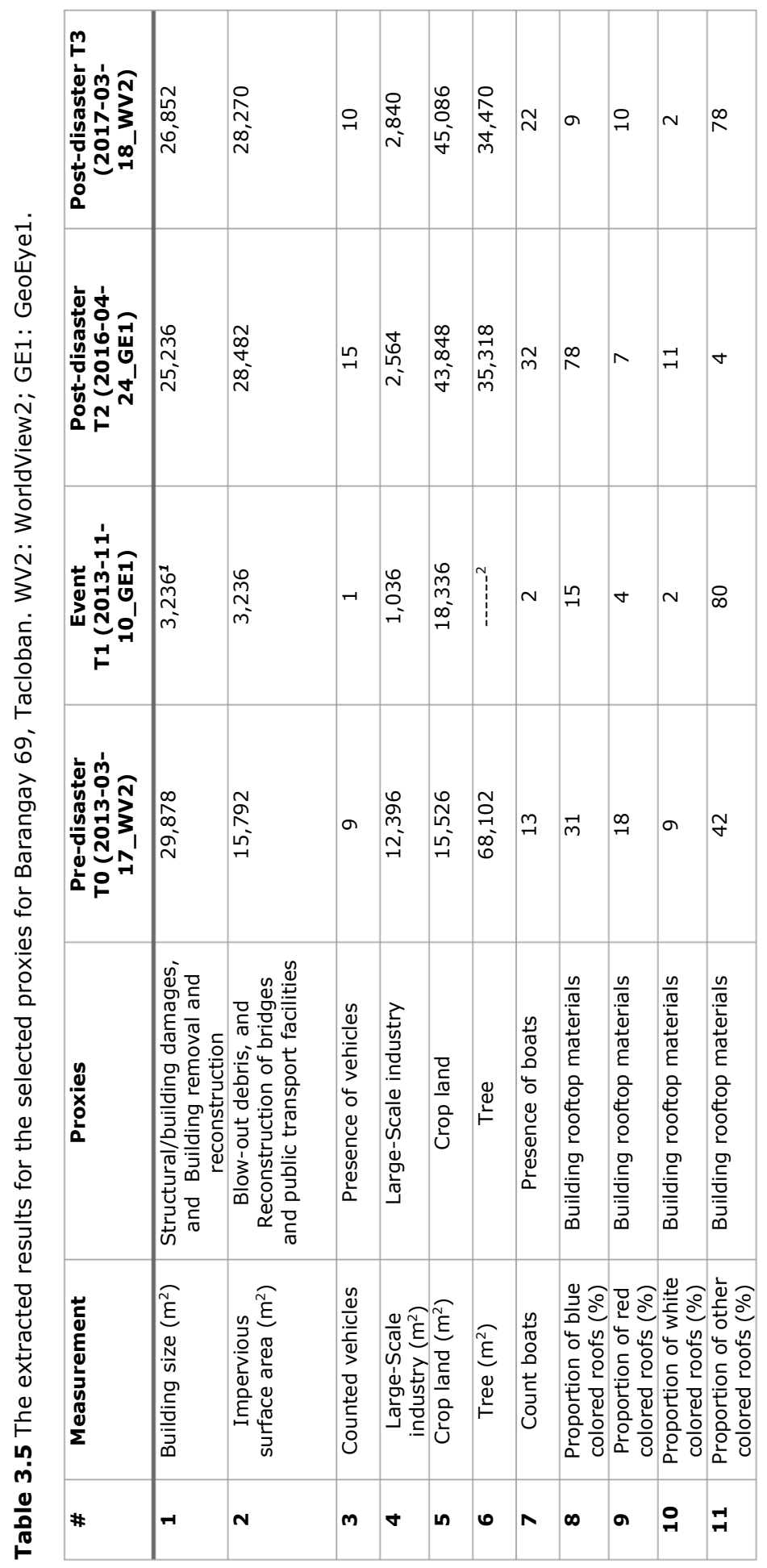




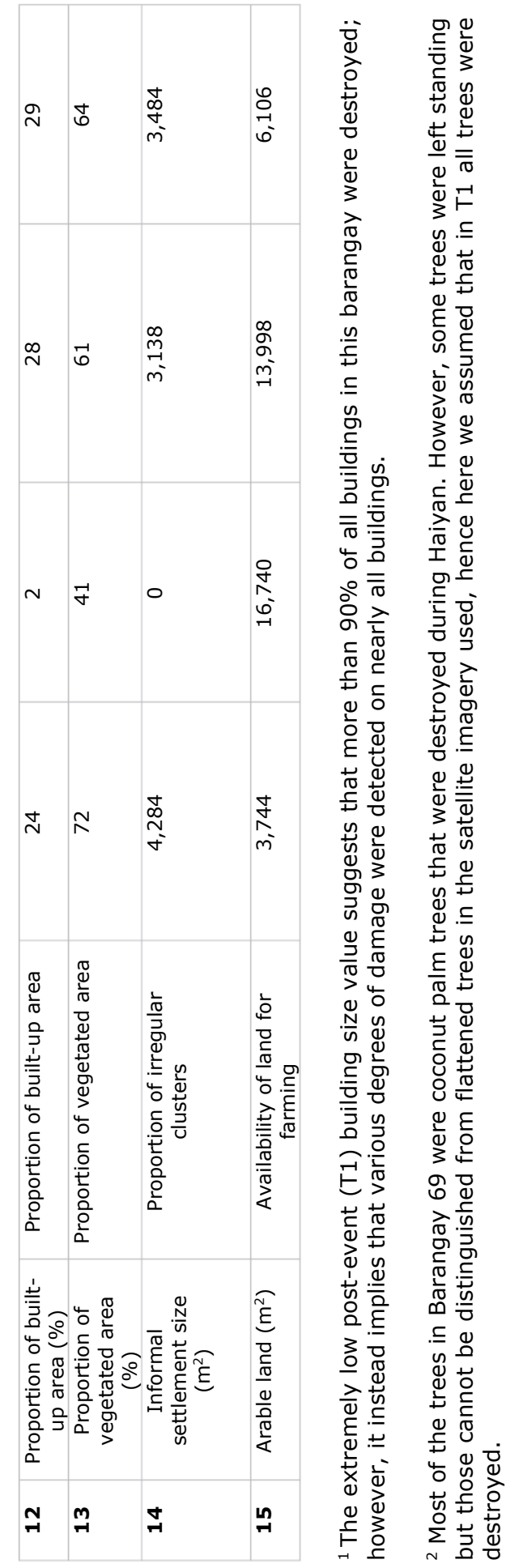




\subsection{Discussion}

The underlying assumption in this work was that communities that received DRR-related interventions such as comprehensive land-use planning would perform better in the aftermath of a major disaster than those without, and we considered post-disaster recovery to be a proxy of resilience. The analysis of high spatial resolution satellite images for 18 barangays provided a detailed picture of LCLU changes caused by typhoon Haiyan and the recovery efforts during the subsequent 3 years. The developed conceptual framework based on a range of proxy indicators primarily extracted through ML allowed the quantification of reconstruction in both formal and informal settlements, as well as of industrial facilities, but also changes in agricultural LU. As such we demonstrated the utility of RS to detect and characterize recovery, similar to (Sheykhmousa et al. 2019), who did this for the city of Tacloban only. Nevertheless, the inherently descriptive nature and resulting limitation of RS data became apparent, in that the reasons for the spatially and temporally highly variable recovery performance observed could not be determined. Survey data are suitable to generate very detailed insights into DRR-related perspectives, motivations and drivers of the affected population, though their acquisition required a considerable effort and planning horizon. In addition, socio-economic data are frequently limited when it comes to assessing physical characteristics such as infrastructural changes. While the survey data are able to reveal changes in DRR-related perceptions and motivations of humans, they are not suitable to estimate actual recovery processes such as building reconstruction or industry recovery.

However, while the image analysis does provide detailed information about LC and LU changes directly linked to recovery, the observed recovery performance could not always be unambiguously explained by the socioeconomic data. For example, contrary to expectation we observed slower recovery rates in barangays that had received the SIMPLE intervention or other donor support, but also for those communities with prior disaster experience. This may be the consequence of more rigorous planning efforts and stricter building policies. As such slower recovery may signal higher quality of the newly established building stock and could indicate higher resilience, including to future events. Nevertheless, this does not agree with the generally accepted notion of resilient communities showing faster recovery (Ghaffarian et al. 2018; Sheykhmousa et al. 2019). The image analysis did show an overall rapid and positive recovery, with building stock getting reconstructed quickly (although more rapidly in urban than in rural communities), with the proportion of informal settlements declining, and some adaptation in the agricultural sector, such as vulnerable coconut palms getting replaced by other crops. 
The RS analysis is subject to additional limitations. The quantification of recovery hinges on an accurate initial damage assessment. However, RS-based damage detection continues to pose challenges, even if very high resolution images are used (Kerle 2010, 2015b), and in particular when volunteers carry out the immediate post-disaster damage mapping (Kerle 2015a; Kerle and Hoffman 2013). In particular in Tacloban the blanket coverage with washedup or wind-blown rubble and debris led to strong damage overestimation in published maps (Ghaffarian and Kerle 2019). An analysis of the damage map created during a Humanitarian OpenStreetMap campaign showed an overestimation of destroyed buildings in Tacloban by some $92 \%$ (Westrope et al. 2014), and also the damage and resulting recovery maps in (Ghaffarian et al. in press; Sheykhmousa et al. 2019) show this overestimation to some extent. It is likely that some of the barangay that in this study show building reconstruction rates of $>100 \%$ within about 3 years also suffered less actual building damage. In addition some of the proxies used in the analysis, in particular LU classes such as crop or tree types, but also related to building and settlement type, suffer from uncertainties. While it would also have been ideal to process images covering all 100 DEval municipalities, this would have been prohibitive in terms of image and processing costs. The narrow coastal strip from Tacloban to Dulag alone covers an area of approximately $400 \mathrm{~km}^{2}$. A single high resolution multispectral Pléiades images for this area would cost in excess of 5,000 US\$, while an 8-band WorldView-3 image would cost about 7,500 US $\$$, and a simple recovery assessment requires 3 time steps as a minimum (T0, T1, T2). For the image analysis part of this work we made use of image data costing approximately 45,000 US $\$$, part of which was donated by the Digital Globe foundation. In particular immediate post-event images are also often acquired at large off-nadir angles, resulting in additioanl challenges for detailed recovery assessment (Ghaffarian et al. in press).

\subsection{Conclusions}

We initially hypothesized that image data could add critical additional information to the questionnaire-based evaluation, and might allow an extrapolation to areas with fewer or no interviews. However, it became clear quickly that matching a RS analysis to the spatial extent of the DEval study (100 municipalities spread over 2 regions) was prohibitive. In addition to cost, many of the more outlying communities are not adequately covered in high resolution data catalogues. Conversely, the statistical analysis in this study showed that it was actually the survey information that added value to the interpretation of the LCLU classification results. Similar to other recent work (e.g., Sheykhmousa et al. 2019) the limitations of RS to explain spatially and temporally variable recovery also became clear here, and the field-based information proved valuable. 
The purpose of this study was to develop a conceptual framework for postdisaster recovery and resilience assessment using remote sensing. Subsequently the usefulness of the RS, in particular satellite images, was evaluated to assess the resilience-centered development interventions. The image-based proxies extracted from LC LU classification results were employed to assess the recovery process and thus resilience, assuming the resilient municipalities recover quicker. However, regarding the utility of RS data to support intervention evaluations in such large areas, current limitations are clear. Vast amounts of detailed image data are necessary, resulting also in high processing cost. However, the efficiency and sophistication of damage- and recovery-focused processing has also been increasing, in particular through advances in deep learning/ CNN (Duarte et al. in press; Ghaffarian et al. in press), where a trend towards automatically handling large datasets as training samples is evident. The recent recovery study that focused on Tacloban (Sheykhmousa et al. 2019) showed that a detailed LU classification that is needed for comprehensive urban recovery assessment requires very high spatial resolution imagery. However, it also showed that lower resolution data suffice for a LC assessment in urban areas, and are also suitable to support the quantification of recovery in rural areas, where the size of relevant objects tends to be larger. This means that a more optimized use of images can be achieved, i.e. very high spatial resolution imagery is not always needed.

One of the critical aspects related to resilience is climate change adaptation. This can include physical measures such as dikes or dams, but increasingly nature-based solutions are sought (Lafortezza and Sanesi 2019; Zwierzchowska et al. 2019), and remote sensing has shown its utility in monitoring many relevant indicators (Taramelli et al. 2019). In coastal communities such as the ones studied in this work, which are subject to rising sea levels, salt water intrusion and regular tropical storms, image data have tremendous potential in detecting and quantifying adaptive measures, such as vulnerable crops (e.g., coconut palms) getting replaced by more resilient solutions, or protective mangrove corridors getting reinstated. Evidence of such actions can be a valuable addition to household surveys. For rural areas and crop monitoring it may be possible to work with freely available data, such as $3-5 \mathrm{~m}$ resolution images from Planet. However, more work is needed in this area.

Due to the high costs for image data and high demand on human resources (coding and manual data processing), we were unable to compute LC and LU for the full sample of municipalities $(\mathrm{N}=100)$ for which socioeconomic survey data were available via the DEval impact evaluation. Since we purposefully selected seven municipalities (13-18 barangays), our analysis did not permit a rigorous attribution of causal impacts. Nevertheless, the purpose of this work was to provide a comprehensive theoretical scheme for post-disaster recovery 
and resilience assessments using RS. Considering the above-explained limitations of the RS, future research may employ integration of the survey data with RS for a comprehensive assessments. For instance, using a geospatial impact evaluation designs (GIE) (Benyishay et al. 2017), and use matching techniques to obtain a sufficiently large sample of control and intervention barangays.. With a sufficiently large sample the full potential of combining survey information with RS data could then be realized.

\subsection{References of Chapter 3}

Acda, M. N., 2015. Fuel pellets from downed coconut (Cocos nucifera) in super typhoon Haiyan. Biomass \& Bioenergy, 83, 539-542.

Arciniegas, G., Bijker, W., Kerle, N., Tolpekin, V. A., 2007. Coherence- and amplitude-based analysis of seismogenic damage in Bam, Iran, using Envisat ASAR data. Ieee Transactions on Geoscience and Remote Sensing, 45(6(1) Special issue "Remote sensing for major disaster prevention, monitoring and assessment"), 1571-1581.

Bengtsson, L., Hodges, K. I., Roeckner, E., 2006. Storm tracks and climate change. Journal of Climate, 19(15), 3518-3543.

Benyishay, A., Runfola, D., Tricher, R., Dolan, C., Goodman, S., Parks, B., Tanner, J., Heuser, S., Batra, G., Anand, A., 2017. A primer on geospatial impact evaluation methods, tools, and applications. Retrieved from Williamsburg, VA, USA:

Berke, P. R., J., K., D., W., 1993. Recovery after Disaster: Achieving Sustainable Development, Mitigation and Equity. Disasters, 17(2).

Bevington, J., Pyatt, S., Hill, A., Honey, M., Adams, B., Davidson, R., Brink, S., Chang, S., Panjwani, D., Mills, R., Amyx, P., Eguchi, R., 2010. UNCOVERING COMMUNITY DISRUPTION USING REMOTE SENSING: AN ASSESSMENT OF EARLY RECOVERY IN POST-EARTHQUAKE HAITI. Miscellaneous Report \#69, University of Delaware (Newark, Delaware USA), Disaster Research Center.

Boschetti, M., Nelson, A., Nutini, F., Manfron, G., Busetto, L., Barbieri, M., Laborte, A., Raviz, J., Holecz, F., Mabalay, M. R. O., Bacong, A. P., Quilang, E. J. P., 2015. Rapid assessment of crop status: An application of MODIS and SAR Data to rice areas in Leyte, Philippines affected by Typhoon Haiyan. Remote Sensing, 7(6), 6535-6557.

Bradshow, S., 2004. Socio-economic impacts of natural disaster: a gender analysis. Nation Economic Commission for Latin America (ECLA), Serie Manuales 32.

Brown, D., Platt, S., Bevington, J., Saito, K., Adams, B., Chenvidyakarn, T., Spence, R., Chuenpagdee, R., Khan, A., 2015. Monitoring and evaluating post-disaster recovery using high-resolution satellite imagery - towards standardised indicators for post-disaster recovery.

Burton, C., Mitchell, J. T., Cutter, S. L., 2011. Evaluating post-Katrina recovery in Mississippi using repeat photography. Disasters, 35(3), 488-509.

Burton, C. G., 2012. The development of metrics for community resilience to Natural disasters. University of South Carolina, University of South Carolina. 
Chan, J. C.-W., Paelinckx, D., 2008. Evaluation of Random Forest and Adaboost tree-based ensemble classification and spectral band selection for ecotope mapping using airborne hyperspectral imagery. Remote Sensing of Environment, 112(6), 2999-3011.

Chen, C., Zhang, B., Su, H., Li, W., Wang, L., 2015. Land-use scene classification using multi-scale completed local binary patterns. Signal, Image and Video Processing, 10(4), 745-752.

Chen, T., Guestrin, C., 2016. XGBoost: A Scalable Tree Boosting System. Paper presented at the Proceedings of the 22nd ACM SIGKDD International Conference on Knowledge Discovery and Data Mining, San Francisco, California, USA.

Coppola, D. P., 2015. Chapter 1 - The Management of Disasters Introduction to International Disaster Management (Third Edition) (pp. 1-39). Boston: Butterworth-Heinemann.

Daniell, J., Mühr, B., Girard, T., Dittrich, A., Fohringer, J., Lucas, C., KunzPlapp, T., 2013. Super Typhoon Haiyan/Yolanda - report no. 2. Retrieved from Karlsruhe Institute of Technology, Germany:

Duarte, D., Nex, F., Kerle, N., Vosselman, G., in press. Detection of seismic façade damages with multi-temporal oblique aerial imagery. Isprs International Journal of Geo-Information.

Ebert, A., Kerle, N., Stein, A., 2009. Urban social vulnerability assessment with physical proxies and spatial metrics derived from air- and spaceborne imagery and GIS data. Natural Hazards, 48(2), 275-294.

Flax, L., Jakson, R., Stein, D., 2002. Community vulnerability assessment tool methodology.

Friedman, J. H., 2001. Greedy function approximation: A gradient boosting machine. Ann. Statist., 29(5), 1189-1232.

Georganos, S., Grippa, T., Vanhuysse, S., Lennert, M., Shimoni, M., Kalogirou, S., Wolff, E., 2018a. Less is more: optimizing classification performance through feature selection in a very-high-resolution remote sensing objectbased urban application. GIScience \& Remote Sensing, 55(2), 221-242.

Georganos, S., Grippa, T., Vanhuysse, S., Lennert, M., Shimoni, M., Wolff, E., 2018b. Very High Resolution Object-Based Land Use-Land Cover Urban Classification Using Extreme Gradient Boosting. IEEE Geoscience and Remote Sensing Letters, 15(4), 607-611.

Gertler, P. J., Martinez, S., Premand, P., Rawlings, L., Vermeersch, C., 2016. Impact evaluation in practice (Second edition ed.). Washington, DC, USA: World Bank Group.

Ghaffarian, S., Kerle, N., 2019. Towards post-disaster debris identification for precise damage and recovery assessments from UAV and satellite images. Int. Arch. Photogramm. Remote Sens. Spatial Inf. Sci., XLII-2/W13, 297302.

Ghaffarian, S., Kerle, N., Filatova, T., 2018. Remote sensing-based proxies for urban disaster risk management and resilience: A review. Remote Sensing, 10(11), 30.

Ghaffarian, S., Kerle, N., Pasolli, E., Jokar Arsanjani, J., in press. Post-disaster building database updating using automated deep learning: An integration of pre-disaster OpenStreetMap and multi-temporal satellite data. Remote Sensing.

Haas, J. E., Kates, R. W., Bowden, M. J., 1977. Reconstruction following disaster. Cambridge, MA: The MIT Press. 
Hsiang, S., Kopp, R., Jina, A., Rising, J., Delgado, M., Mohan, S., Rasmussen, D. J., Muir-Wood, R., Wilson, P., Oppenheimer, M., Larsen, K., Houser, T., 2017. Estimating economic damage from climate change in the United States. Science, 356(6345), 1362-1368.

Kerle, N., 2010. Satellite-based damage mapping following the 2006 Indonesia earthquake--How accurate was it? International Journal of Applied Earth Observation and Geoinformation, 12(6), 466-476.

Kerle, N., 2015a. Disaster mapping by citizens is limited. Nature, 517(7535), 438-438.

Kerle, N., 2015b. Disasters : risk assessment, management, and post disaster studies using remote sensing. In: Remote sensing of water resources, disasters, and urban studies / edited by P.S. Thenkabail. Boca Raton: CRC Press, 2015. ISBN: 978-1-4822-1792-6 (Remote sensing handbook, 3) pp. 455-481.

Kerle, N., Hoffman, R. R., 2013. Collaborative damage mapping for emergency response: the role of Cognitive Systems Engineering. Natural Hazards and Earth System Sciences, 13(1), 97-113.

Kharin, V. V., Zwiers, F. W., Zhang, X. B., Hegerl, G. C., 2007. Changes in temperature and precipitation extremes in the IPCC ensemble of global coupled model simulations. Journal of Climate, 20(8), 1419-1444.

Kuffer, M., Pfeffer, K., Sliuzas, R., 2016. Slums from Space-15 Years of Slum Mapping Using Remote Sensing. Remote Sensing, 8(6), 455.

Kure, S., Jibiki, Y., Quimpo, M., Manalo, U. N., Ono, Y., Mano, A., 2016. Evaluation of the characteristics of human loss and building damage and reasons for the magnification of damage due to Typhoon Haiyan. Coastal Engineering Journal, 58(1), 27.

Lafortezza, R., Sanesi, G., 2019. Nature-based solutions: Settling the issue of sustainable urbanization. Environmental Research, 172, 394-398.

Lagmay, A. M. F., Agaton, R. P., Bahala, M. a. C., Briones, J., Cabacaba, K. M. C., Caro, C. V. C., Dasallas, L. L., Gonzalo, L. a. L., Ladiero, C. N., Lapidez, J. P., Mungcal, M. T. F., Puno, J. V. R., Ramos, M., Santiago, J., Suarez, J. K., Tablazon, J. P., 2015. Devastating storm surges of Typhoon Haiyan. International Journal of Disaster Risk Reduction, 11, 1-12.

Lech, M., Harten, S., Uitto, J. I., Batra, G., Anand, A., 2018. Improving International Development Evaluation through Geospatial Data and Analysis. International Journal of Geospatial and Environmental Research, 5(2).

Leppert, G., Hohfeld, L., Lech, M., Wencker, T., 2018. Impact, diffusion and scaling-up of a comprehensive land-use planning approach in the Philippines. From development cooperation to national policies. Retrieved from

Bonn: http://www.deval.org/files/content/Dateien/Evaluierung/Berichte/2018/ DEval-2018_Philippinen_final_web.pdf

Ma, L., Li, M., Ma, X., Cheng, L., Du, P., Liu, Y., 2017. A review of supervised object-based land-cover image classification. ISPRS Journal of Photogrammetry and Remote Sensing, 130, 277-293.

Maxwell, A. E., Warner, T. A., Fang, F., 2018. Implementation of machinelearning classification in remote sensing: an applied review. International Journal of Remote Sensing, 39(9), 2784-2817. 
Mboga, N., Persello, C., Bergado, J., Stein, A., 2017. Detection of Informal Settlements from VHR Images Using Convolutional Neural Networks. Remote Sensing, 9(11), 1106.

Mcdem. 2005. Focus on Recovery: A Holistic Framework for Recovery in New Zealand. MCDEM, New Zealand.

Mochizuki, J., Naqvi, A., 2019. Reflecting disaster risk in development indicators. Sustainability, 11(4), 14.

Morrow, B. H., 1999. Identifying and Mapping Community Vulnerability. Disasters, 23(1), 1-18.

Mueller, M., Segl, K., Heiden, U., Kaufmann, H., 2006. Potential of HighResolution Satellite Data in the Context of Vulnerability of Buildings. Natural Hazards, 38(1-2), 247-258.

Nawrotzki, R., 2019. The geodata decision tree: Using geodata for evaluations. Retrieved from Bonn, Germany:

Nery, T., Sadler, R., Solis-Aulestia, M., White, B., Polyakov, M., Chalak, M., 2016, 10-15 July 2016. Comparing supervised algorithms in Land Use and Land Cover classification of a Landsat time-series. Paper presented at the 2016 IEEE International Geoscience and Remote Sensing Symposium (IGARSS).

Pech, L., 2017. Development of an urban area of limited statehood observed from above: The case of Goma, Democratic Republic of the Congo Measuring Statehood on a Sub-National Level: A dialogue among methods (pp. 27-34). Berlin, Germany: Collaborative Research Center (SFB) 700.

Rau, J. Y., Chen, L. C., Liu, J. K., Wu, T. H., 2007. Dynamics monitoring and disaster assessment for watershed management using time-series satellite images. Ieee Transactions on Geoscience and Remote Sensing, 45(6), 1641-1649.

Reif, M. K., Macon, C. L., Wozencraft, J. M., 2011. Post-Katrina land cover, elevation, and volume change assessment along the South shore of Lake Pontchartrain, Louisiana, USA. Journal of Coastal Research, 30-39.

Ren, X., Guo, H., Li, S., Wang, S., Li, J., 2017. A Novel Image Classification Method with CNN-XGBoost Model. 10431, 378-390.

Rose, A., Krausmann, E., 2013. An economic framework for the development of a resilience index for business recovery. International Journal of Disaster Risk Reduction, 5, 73-83.

Rubin, C., Spence, R., Going, C., Markus, M., 1985. Community recovery from a major natural disaster. Institute of Behavioral science University of Colorado Boulder, co, Monograph 41.

Saito, K., Spence, R. J. S., Going, C., Markus, M., 2004. Using High-Resolution Satellite Images for Post-Earthquake Building Damage Assessment: A Study Following the 26 January 2001 Gujarat Earthquake. Earthquake Spectra, 20(1), 145-169.

Sheykhmousa, M., Kerle, N., Kuffer, M., Ghaffarian, S., 2019. Post-disaster recovery assessment with machine learning-derived land cover and land use information. Remote Sensing, 11(10), 1174.

Singh, A., 1989. Review Article Digital change detection techniques using remotely-sensed data. International Journal of Remote Sensing, 10(6), 989-1003.

Takagi, H., Esteban, M., 2016. Statistics of tropical cyclone landfalls in the Philippines: unusual characteristics of 2013 Typhoon Haiyan. Natural Hazards, 80(1), 211-222. 
Taramelli, A., Lissoni, M., Piedelobo, L., Schiavon, E., Valentini, E., Xuan, A. N., Gonzalez-Aguilera, D., 2019. Monitoring green infrastructure for natural water retention using Copernicus global land products. Remote Sensing, 11(13), 27.

The Humanitarian Data Exchange. $2019 . \quad$ Retrieved from https://data.humdata.org/dataset/philippines-administrative-levels-0-to3

The World Bank. 2017. Results Brief - Climate insurance. Retrieved from https://www.worldbank.org/en/results/2017/12/01/climate-insurance

Tol, R. S. J., 2018. The economic impacts of climate change. Review of Environmental Economics and Policy, 12(1), 4-25.

Townshend, I., Awosoga, O., Kulig, J., Fan, H., 2014. Social cohesion and resilience across communities that have experienced a disaster. Natural Hazards, 76(2), 913-938.

UNISDR. 2015a. Global assessment report on disaster risk reduction. Retrieved from https://www.preventionweb.net/english/hyogo/gar/2015/en/home/

UNISDR. 2015b, 14-18 March. Sendai framework for disaster risk reduction 2015 - 2030. Paper presented at the Third World Conf. Disaster Risk Reduction, Sendai, Japan.

Westrope, C., Banick, R., Levine, M., 2014. Groundtruthing OpenStreetMap building damage assessment. In A. Vidan \& D. Shoag (Eds.), Humanitarian Technology: Science, Systems and Global Impact 2014 (Vol. 78, pp. 2939). Amsterdam: Elsevier Science Bv.

Yang, C., Rottensteiner, F., Heipke, C., 2018. Classification of Land Cover and Land Use Based on Convolutional Neural Networks. ISPRS Annals of Photogrammetry, Remote Sensing and Spatial Information Sciences, IV3, 251-258.

Zhang, F., Du, B., Zhang, L., 2016. Scene Classification via a Gradient Boosting Random Convolutional Network Framework. IEEE Transactions on Geoscience and Remote Sensing, 54(3), 1793-1802.

Zhu, Y., Newsam, S., 2015. Land use classification using convolutional neural networks applied to ground-level images. 1-4.

Zwierzchowska, I., Fagiewicz, K., Ponizy, L., Lupa, P., Mizgajski, A., 2019. Introducing nature-based solutions into urban policy - facts and gaps. Case study of Poznan. Land Use Policy, 85, 161-175. 
Chapter 4 - Towards post-disaster debris identification for precise damage and recovery assessments from UAV and satellite images ${ }^{3}$

3 This chapter is based on:

- Ghaffarian, S.; Kerle, N., 2019. Towards post-disaster debris identification for precise damage and recovery assessments from UAV and satellite images. Int. Arch. Photogramm. Remote Sens. Spatial Inf. Sci., XLII-2/W13, 297-302. 


\section{Abstract}

Often disasters cause structural damages and produce rubble and debris, depending on their magnitude and type. The initial disaster response activity is evaluation of the damages, i.e., the creation of a detailed damage estimation for different object types throughout the affected area. First responders and government stakeholders require the damage information to plan rescue operations and later on to guide the recovery process. Remote sensing, due to its agile data acquisition capability, synoptic coverage, and low cost, has long been used as a vital tool to collect information after a disaster and conduct damage assessment. To detect damages from remote sensing imagery (both UAV and satellite images) structural rubble/debris has been employed as a proxy to detect damaged buildings/areas. However, disaster debris often includes vegetation, sediments, and relocated personal property in addition to structural rubble, i.e., items that are wind- or waterborne and not necessarily associated with the closest building. Traditionally, land cover classificationbased damage detection has been categorizing debris as damaged areas. However, in particular, in a waterborne disaster such as tsunamis or storm surges, vast areas end up being debris-covered, effectively hindering actual building damage to be detected, and leading to an overestimation of the damaged area. Therefore, to perform a precise damage assessment, and consequently, recovery assessment that relies on a clear damage benchmark, it is crucial to separate actual structural rubble from ephemeral debris. In this study two approaches were investigated for two types of data (i.e., UAV and multi-temporal satellite images). To do so, three textural analysis, i.e., Gabor filters, Local Binary Pattern (LBP), and Histogram of the Oriented Gradients (HOG), were implemented on mosaic UAV images, and the relation between debris type and their time of removal was investigated using very highresolution satellite images. The results showed that the HOG features, among other texture features, have the potential to be used for debris identification. In addition, multi-temporal satellite image analysis showed that debris removal time needs to be investigated using daily images because the removal time of debris may change based on the type of disaster and its location. 


\subsection{Introduction}

Building/structural damage assessment is a crucial task at the first stage for the post-disaster response phase by supporting rescue operations, and then recovery phase by providing key information to support governments and decision-makers planning for reconstructions.

Remote sensing has been demonstrated as an essential and efficient tool for a rapid damage assessment after a disaster (Brunner et al. 2010). In addition, advances in computer vision and photogrammetry allow scientists to develop complicated but advanced methods. Several studies were conducted for damage assessment using remote sensing data such as UAV (Cotrufo et al. 2018; Galarreta et al. 2015; Vetrivel et al. 2017; Vetrivel et al. 2016) and satellite images (Duarte et al. 2018b; Gillespie et al. 2007; Joshi et al. 2017). Most of them are based on the assumption that the urban disaster debris belongs to building rubbles, and the presence of the debris surrounding and inside/on the buildings has been used as a proxy to extract damage ratio (Kerle and Hoffman 2013). For example, Galarreta et al. (2015) used rubble piles as a damage feature to identify the damage ratio to the buildings from 3D point clouds derived from UAV images. Vetrivel et al. (2015) detected rubble piles/debris around/on/inside buildings in addition to gaps to extract damaged regions of the structures. In another study, Vetrivel et al. (2016) developed a method to detect building damage corresponding to debris, rubble piles, and heavy spalling buildings. In addition, Ural et al. (2011) developed a method for larger urban area damage extraction using very high-resolution satellite images and LiDAR data and showed the efficiency of their method in extracting damaged buildings and their footprint. For a precise damage detection (Duarte et al. 2018a; Duarte et al. 2018b) fused satellite and UAV images using a CNNbased approach and improved the accuracy of the rubble/debris-based damage identification results. All of the aforementioned studies extracted the building damages with a high success/accuracy rate via mainly extracting the rubble piles/debris. However, disaster debris often incorporates sediments, vegetative debris, and personal property in addition to building materials/rubble, which is not necessarily belong to the closest building. For example, in a tsunami/storm surge scenario, a large quantity of mixed debris can be washed up close to the intact buildings, or a high-speed wind can rip roofs off houses and pluck tree fronds, and relocate them during the event. Furthermore, to do a post-disaster recovery assessment, damage assessment is needed as the first step to determine the damaged areas and ratios. Changes in land cover and land use of the areas were mostly used for damage and, consequently, recovery assessment of the areas (Ghaffarian et al. 2018; Ishihara and Tadono 2017). Hence, land cover/use classification/change detection of post-disaster satellite images the debris class is mostly used as an indicator/proxy of the damaged building and roads/area (Ghaffarian et al. 2018). For example, land cover- 

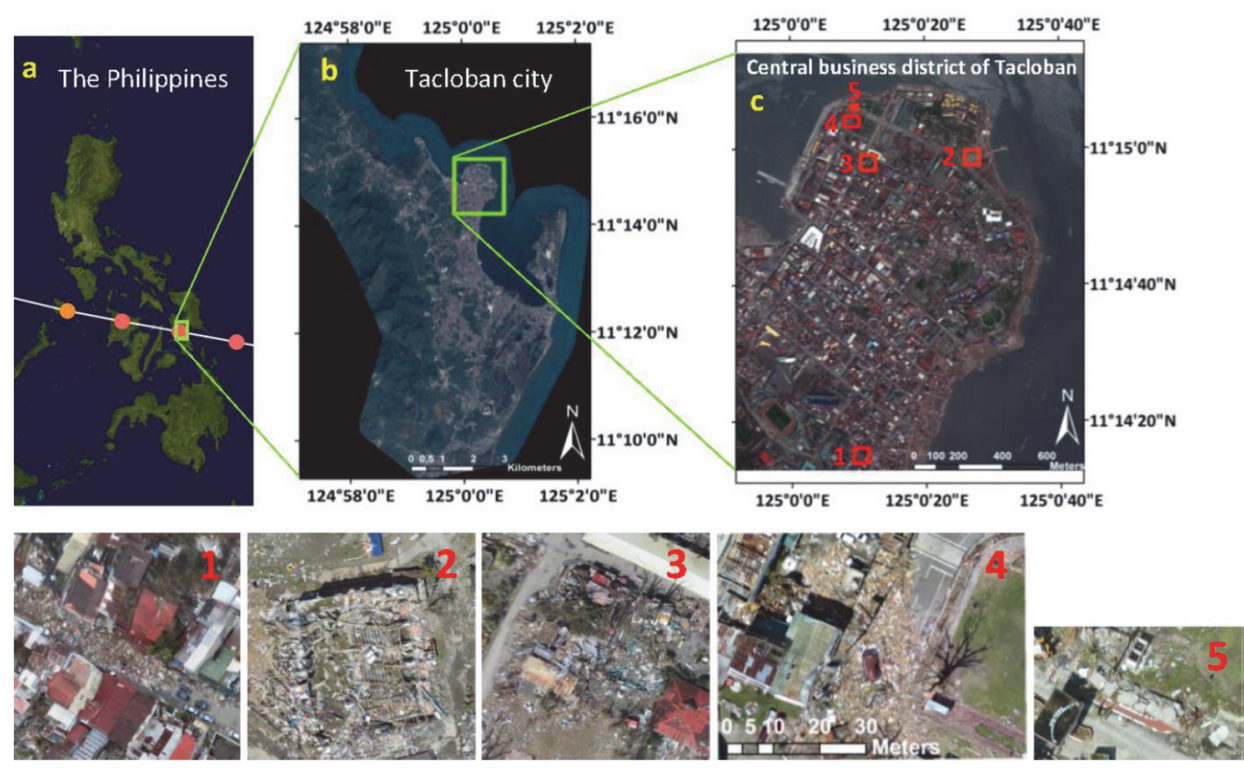

Figure 4.1 a) Track of Typhoon Haiyan over the Philippines, b-c) Overview of Tacloban city. 1-5) The selected UAV images for one week after the disaster from the study area.

based change detection derived from high-resolution satellite imagery and conducted a land cover and land use assessments for a few days after a disaster. They demonstrated that the remobilized debris in the entire area caused inaccuracies in land cover and land use classification results. Land cover and land use classification of the damaged area, particularly in water-related disasters, are prone to overestimation due to washed-up debris that may hinder the intact road and structures and also lead to overestimation of the post-disaster damaged area/ratio. Hence, identification of the disaster debris types is critical for precise post-disaster damage and consequently recovery assessments from both UAV and satellite images.

In this study, we aim to address the challenge of post-disaster debris identification by investigating the potential of the UAV/drone images and multitemporal very high-resolution satellite images acquired some days, weeks and months after a disaster to distinguish between quasi-permanent debris (e.g., rubble related to building materials) and ephemeral materials that get continuously remobilized (e.g., flotsam deposited by flood/storm surge water, and wind-blown vegetation matter such as palm fronds) (figure 4.1).

\subsection{Methodology}

Two distinct approaches are proposed in this paper to identify the debris types for after disaster situations. UAV images due to providing very high-resolution images can contribute to the identification of the disaster debris types. Thus, we conducted a textural analysis using the UAV images to compare areas with 
quasi-permanent debris and ephemeral materials and figure out the most informative ones to distinguish them. In the second approach, the idea that the ephemeral materials can be collected much earlier than the structural debris is investigated to identify the debris types from multi-temporal satellite images.

\subsubsection{Textural analysis}

In order to distinguish the quasi-permanent structural rubble from other more remobilized debris from UAV images, three textural analyses were investigated, i.e., Local Binary Patterns (LBPs), Gabor features, and Histogram of the Oriented Gradients (HOG).

\subsubsection{Texture features (LBPs and Gabor features)}

In general, image-based textural features are extracted using two approaches; statistical-based and signal processing-based approaches. Statistical methods make use of statistical relations of the spatial distribution of gray-level brightness values within the image. Currently, the gray-level co-occurrence matrix (GLCM) is one of the well-known statistical-based methods for textural analysis, which is also used as a basis for other advanced textural methods (e.g., Local Binary Pattern). GLCM features are used for different remote sensing application such as land use classification (Kabir et al. 2010; Pacifici et al. 2009), slum area detection (Kuffer et al. 2016b), built-up area extraction (Pesaresi et al. 2008), and high-resolution satellite image analysis (Zhang et al. 2017). GLCM-based Local Binary Patterns (LBP) have been indicated as one of the most useful and powerful texture analyzing for high-resolution remote sensing images due to their computational simplicity and discriminative power (Gevaert et al. 2016; Kuffer et al. 2016a; Mboga et al. 2017).

GLCM features (e.g., entropy, mean, correlation, homogeneity) are computed based on the occurrence of a pair of grey-level pixels in an image in predefined directions (Rao et al. 2002). Local Binary Pattern (LBP) features (Ojala et al. 2002) are computed based on a selected number of neighboring pixels (N) at a defined distance (d) from the central pixel, which is rotationally invariant. LBPs are developed to identify uniform features, such as corners and edges. In this study, LBP features were extracted with $\mathrm{N}=8$ and $\mathrm{d}=16$ from UAV images.

Signal processing-based texture extraction approaches cut-up image data into different frequency components, and use frequency information of the signals in addition to spatial characteristics of the selected image. One of the wellknown, such textural analysis is a wavelet-based method (Arivazhagan et al. 2006). Wavelet-based texture features were also used for remote sensing applications (Vetrivel et al. 2017) and found to be superior to GLCM texture features in many applications, including classification of remote sensing images 
(Ruiz et al. 2004). Furthermore, wavelets and particularly one of its methods, Gabor filters were used for damage assessment from remote sensing imagery (Radhika et al. 2012; Vetrivel et al. 2016). Gabor filters/features, which are based on wavelets, have been indicated as a robust texture extraction method for damage detection (Arivazhagan et al. 2006; Vetrivel et al. 2015, 2016). Gabor features are computed using a set of filters, and each of which is specifically defined to carry out frequency information at a specific orientation. Gabor filters separate image regions based on spatial frequency and orientation. Detailed information about the generation of the Gabor filters and their application are given by Arivazhagan et al. (2006).

Both LBP and Gabor features are used in this study to investigate their usefulness in identifying debris types.

\subsubsection{Histogram of the Oriented Gradients (HOG)}

The HOG is a feature descriptor that is widely used in computer vision and remote sensing for object detection and classification (Dalal and Triggs 2005; Gao et al. 2013; Xiao et al. 2015; Xu and Liu 2016; Xu et al. 2016). The HOG uses the spatial distribution of the gradients in the image regions to measure the spatial variation of edge orientations within a region (Kobayashi et al. 2008), which is a crucial factor in defining/extracting the shape of an object (Dalal and Triggs 2005). The magnitude and angle of the gradient of pixels in the image are computed to extract HOG features. Then the magnitude of the gradients is binned into a histogram according to their angle/orientation for each predefined image block/cell. After normalizing the results of all blocks, they are concatenated to generate the image based on the block size. Furthermore, feature vectors that represent the gradient orientation and magnitude of blocks can be computed from the histograms. The HOG feature vectors were calculated in this study from UAV images to investigate their utility in identifying debris types.

\subsubsection{Multi-temporal analysis}

Since structural rubbles are supposed to be heavier than ephemeral debris, they should be removed easier, and thus earlier, than structural rubbles after a disaster. Hence, the temporal change of the debris deposits in the images are monitored using multi-temporal satellite images. Furthermore, since the size of debris range from very small to bigger objects in the images, very highresolution satellite images with 0.5 spatial resolution are employed.

\subsection{Results and discussions}

The proposed approaches were tested in Tacloban city, the Philippines, which was hit by super Typhoon Haiyan in November 2013. Since the Typhoon Haiyan 
also caused a storm surge during the event, which led to deposit washed-up debris in urban areas, it is suitable to examine the proposed approaches. Five different locations of the debris were selected from the urban area of the Tacloban to implement the proposed approaches and discuss the results (Fig.1).

\subsubsection{Textural analysis of UAV images}

Three textural methods (i.e., HOG, LBP and Gabor filters/magnitude) were implemented on the five selected UAV image regions. From selected images, \#1 and \#4 images mostly include ephemeral debris, while the others mostly consist of the regions with quasi-permanent structural rubbles. The LBP and Gabor features are studied, and no significant difference between debris types are found.
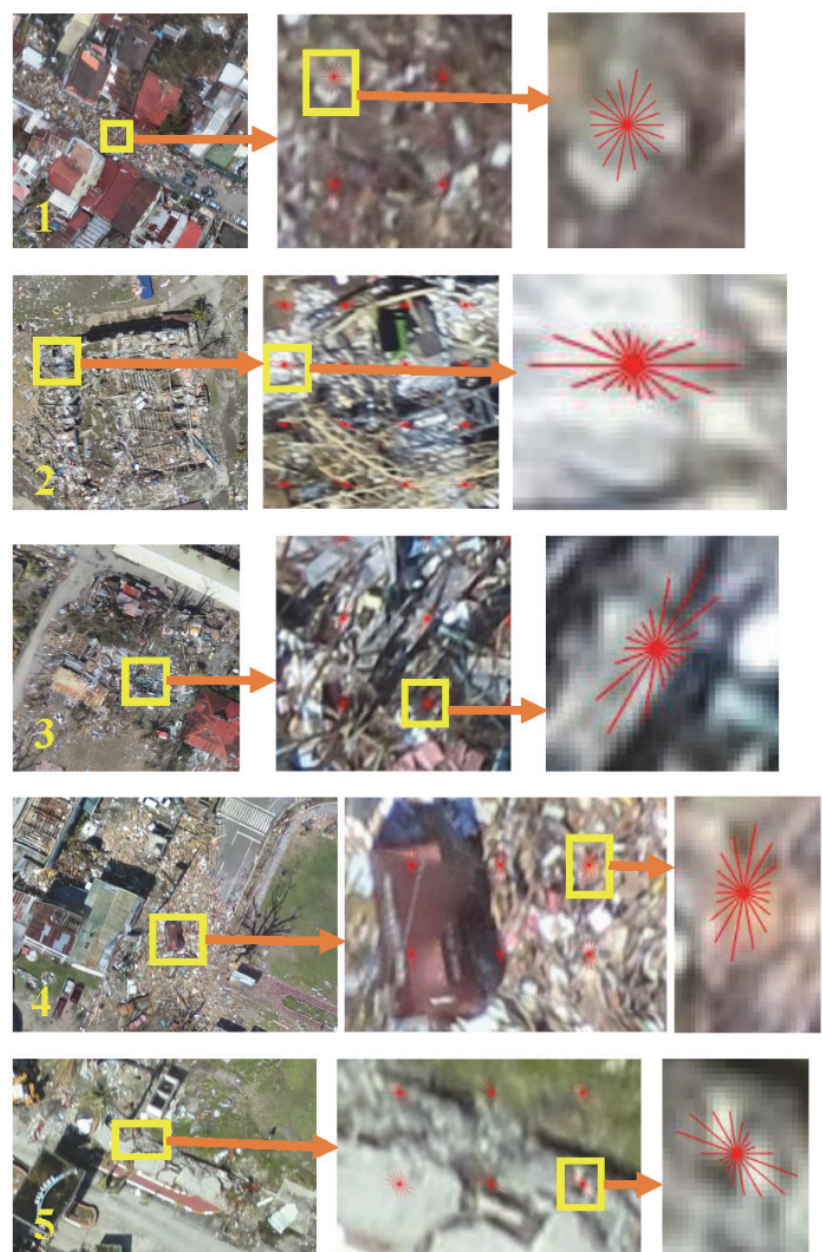

Figure 4.2 1-5) UAV images and their corresponding HOG vector results for the denoted regions. 
Figure 4.2 shows the results of the HOG textural analysis of the selected UAV images. Visual inspection of the results shows a slight difference between HOG of the ephemeral debris and quasi-structural rubble, while ephemeral debris has spread distribution of the gradient orientations (see HOG of images 1 and 2). Structural rubbles due to containing bigger sized objects have less spread HOG distribution. However, visually inspection of the other textural results does not show a significant difference between debris types.

\subsubsection{Multi-temporal analysis of satellite images}

Satellite images acquired by different platforms (e.g., GeoEye1, World_View2 and 3, and Pleiades satellite) for 2, 3, 5, 7 days, 3, 4, 5 weeks and 2, 8 and 9 months after the disaster are analyzed to detect debris changes and extract the relation of temporal changes with debris types in the area.

Figure 4.3 shows the satellite images for the selected area of Tacloban city. Considering the change in the debris area denoted at the images, we can see that most of the debris (on the road) was removed between the first week and 4 weeks after the disaster. However, remaining debris next to the road was removed 5 weeks after the disaster. Since most of the debris had been disappeared from week 4 in the images, we cannot find any relation between the removal time of the debris, and more satellite images are needed to study the changes between the first week and 4 weeks after the disaster. 


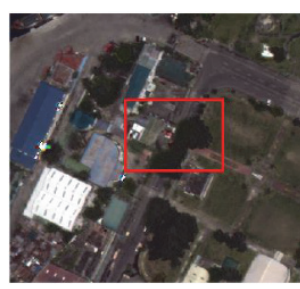

(a) Before the disaster

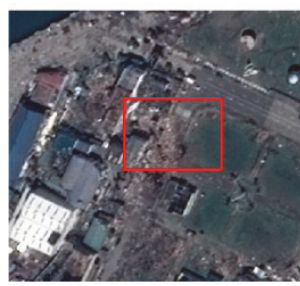

(e) one week after the disaster

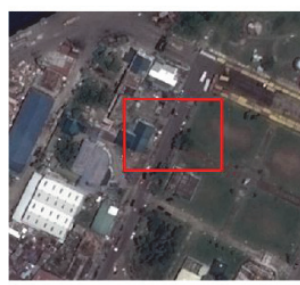

(i) 8 months after the disaster

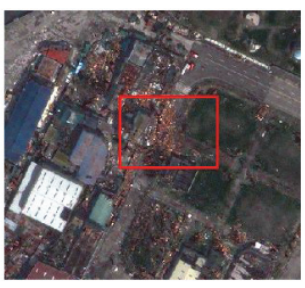

(b) 2 days after the disaster

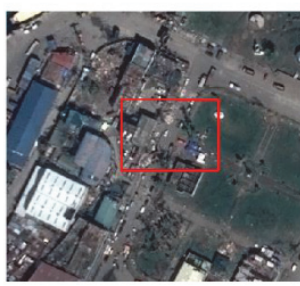

(f) 4 weeks after the disaster

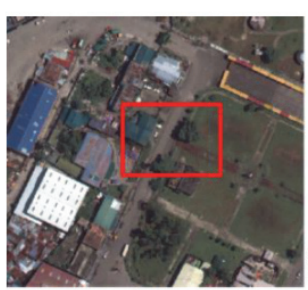

(j) 9 months after the disaster

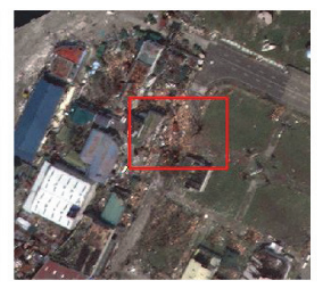

(c) 3 days after the disaster

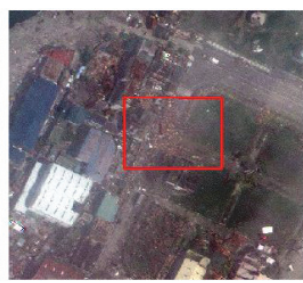

(d) 5 days after the disaster

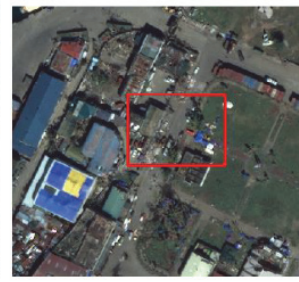

(g) 5 weeks after the disaster

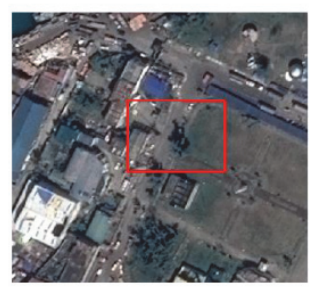

(h) 2 months after the disaster

Figure 4.3 a-j) Very high-resolution satellite images respectively for before disaster, 2, 3, 5, 7 days, 4 and 5 weeks, and 2, 8, and 9 months after the disaster.

\subsection{Conclusions and future work}

In this paper, two approaches are proposed and analyzed for post-disaster type identification. Textural methods are studied for UAV images to test HOG, LBP and Gabor features in differentiating the quasi-permanent structural rubble and ephemeral debris. The results showed that HOG is the most effective feature; however, in the future, to precisely investigate the efficiency of the features and their effectiveness in disaster debris type identification, they should be followed by a classification method and conduct a quantitative comparison. In addition, using 3D point clouds derived from UAV images will help debris identification. Furthermore, the idea of that ephemeral debris due to containing light-weighted debris can be removed earlier than structural rubble is investigated using multi-temporal satellite images. Based on the achieved results in this study and the used time intervals after the disaster, we did not find a strong relationship between the time of removal and debris type. However, it is demonstrated that in order to study this approach, we need daily data/images, type of the structures, and other characteristics of the considered areas that can influence the debris removal time and types. Hence, in the future, this idea should be studied using daily data (e.g., daily drone 
imagery), and use classification methods to extract the disaster debris areas quantitatively.

\subsection{References of Chapter 4}

Arivazhagan, S., Ganesan, L., Priyal, S. P., 2006. Texture classification using Gabor wavelets based rotation invariant features. Pattern Recognition Letters, 27(16), 1976-1982.

Brunner, D., Lemoine, G., Bruzzone, L., 2010. Earthquake damage assessment of buildings using VHR optical and SAR imagery. IEEE Transactions on Geoscience and Remote Sensing, 48(5), 2403-2420.

Cotrufo, S., Sandu, C., Giulio Tonolo, F., Boccardo, P., 2018. Building damage assessment scale tailored to remote sensing vertical imagery. European Journal of Remote Sensing, 51(1), 991-1005.

Dalal, N., Triggs, B., 2005. Histograms of oriented gradients for human detection. Paper presented at the IEEE Computer Society Conference on Computer Vision and Pattern Recognition (CVPR'05), 20-25 June 2005.

Duarte, D., Nex, F., Kerle, N., Vosselman, G., 2018a. Multi-resolution feature fusion for image classification of building damages with convolutional neural networks. Remote Sensing, 10(10), 1636.

Duarte, D., Nex, F., Kerle, N., Vosselman, G., 2018b. Satellite image classification of building damages using airborne and satellite image samples in a deep learning approach. ISPRS Annals of Photogrammetry, Remote Sensing and Spatial Information Sciences, IV-2, 89-96.

Galarreta, J. F., Kerle, N., Gerke, M., 2015. UAV-based urban structural damage assessment using object-based image analysis and semantic reasoning. Natural Hazards and Earth System Sciences, 15(6), 10871101.

Gao, F., Xu, Q., Li, B., 2013. Aircraft detection from VHR images based on circle-frequency filter and multilevel features. The Scientific World Journal, 2013, 917928-917928.

Gevaert, C. M., Persello, C., Sliuzas, R., Vosselman, G., 2016. Classification of Informal Settlements through the Integration of 2D and 3D Features Extracted from Uav Data. ISPRS Annals of Photogrammetry, Remote Sensing and Spatial Information Sciences, III-3, 317-324.

Ghaffarian, S., Kerle, N., Filatova, T., 2018. Remote Sensing-Based Proxies for Urban Disaster Risk Management and Resilience: A Review. Remote Sensing, 10(11), 1760.

Gillespie, T. W., Chu, J., Frankenberg, E., Thomas, D., 2007. Assessment and Prediction of Natural Hazards from Satellite Imagery. Progress in Physical Geography, 31(5), 459-470.

Ishihara, M., Tadono, T., 2017. Land cover changes induced by the great east Japan earthquake in 2011. Scientific Reports, 7, 45769.

Joshi, A. R., Tarte, I., Suresh, S., Koolagudi, S. G., 2017, 19-22 Oct. 2017. Damage identification and assessment using image processing on postdisaster satellite imagery. Paper presented at the 2017 IEEE Global Humanitarian Technology Conference (GHTC).

Kabir, S., He, D. C., Sanusi, M. A., Wan Hussina, W. M. A., 2010. Texture analysis of IKONOS satellite imagery for urban land use and land cover classification. The Imaging Science Journal, 58(3), 163-170. 
Kerle, N., Hoffman, R. R., 2013. Collaborative damage mapping for emergency response: The role of cognitive systems engineering. Natural Hazards and Earth System Science, 13(1), 97-113.

Kobayashi, T., Hidaka, A., Kurita, T., 2008. Selection of Histograms of Oriented Gradients Features for Pedestrian Detection, Berlin, Heidelberg.

Kuffer, M., Pfeffer, K., Sliuzas, R., 2016a. Slums from Space-15 Years of Slum Mapping Using Remote Sensing. Remote Sensing, 8(6), 455.

Kuffer, M., Pfeffer, K., Sliuzas, R., Baud, I., 2016b. Extraction of Slum Areas From VHR Imagery Using GLCM Variance. Ieee Journal of Selected Topics in Applied Earth Observations and Remote Sensing, 9(5), 1830-1840.

Mboga, N., Persello, C., Bergado, J., Stein, A., 2017. Detection of informal settlements from VHR images using Convolutional Neural Networks. Remote Sensing, 9(11), 1106.

Ojala, T., Pietikainen, M., Maenpaa, T., 2002. Multiresolution gray-scale and rotation invariant texture classification with local binary patterns. IEEE Transactions on Pattern Analysis and Machine Intelligence, 24(7), 971987.

Pacifici, F., Chini, M., Emery, W. J., 2009. A neural network approach using multi-scale textural metrics from very high-resolution panchromatic imagery for urban land-use classification. Remote Sensing of Environment, 113(6), 1276-1292.

Pesaresi, M., Gerhardinger, A., Kayitakire, F., 2008. A robust built-up area presence index by anisotropic rotation-invariant textural measure. IEEE Journal of Selected Topics in Applied Earth Observations and Remote Sensing, 1(3), 180-192.

Radhika, S., Tamura, Y., Matsui, M., 2012. Use of post-storm images for automated tornado-borne debris path identification using texture-wavelet analysis. Journal of Wind Engineering and Industrial Aerodynamics, 107108, 202-213.

Rao, P. V. N., Sai, M. V. R. S., Sreenivas, K., Rao, M. V. K., Rao, B. R. M., Dwivedi, R. S., Venkataratnam, L., 2002. Textural analysis of IRS-1D panchromatic data for land cover classification. International Journal of Remote Sensing, 23(17), 3327-3345.

Ruiz, L. A., Fernández-Sarría, A., Recio, J. A., 2004. Texture feature extraction for classification of remote sensing data using wavelet decomposition: A comparative study. Paper presented at the International Archives of the Photogrammetry, Remote Sensing and Spatial Information Sciences, XXXV- B4.

Ural, S., Hussain, E., Kim, K., Fu, C.-S., Shan, J., 2011. Building Extraction and Rubble Mapping for City Port-au-Prince Post-2010 Earthquake with GeoEye-1 Imagery and Lidar Data. Photogrammetric Engineering \& Remote Sensing, 77(10), 1011-1023.

Vetrivel, A., Gerke, M., Kerle, N., Nex, F., Vosselman, G., 2017. Disaster damage detection through synergistic use of deep learning and 3D point cloud features derived from very high resolution oblique aerial images, and multiple-kernel-learning. ISPRS Journal of Photogrammetry and Remote Sensing, 140, 445-59.

Vetrivel, A., Gerke, M., Kerle, N., Vosselman, G., 2015. Identification of damage in buildings based on gaps in 3D point clouds from very high resolution oblique airborne images. ISPRS Journal of Photogrammetry and Remote Sensing, 105, 61-78. 
Vetrivel, A., Gerke, M., Kerle, N., Vosselman, G., 2016. Identification of structurally damaged areas in airborne oblique images using a visual-bagof-words approach. Remote Sensing, 8(3), 231.

Xiao, Z., Liu, Q., Tang, G., Zhai, X., 2015. Elliptic Fourier transformation-based histograms of oriented gradients for rotationally invariant object detection in remote-sensing images. International Journal of Remote Sensing, $36(2), 618-644$.

$\mathrm{Xu}, \mathrm{F}$. , Liu, J.-H., 2016. Ship detection and extraction using visual saliency and histogram of oriented gradient. Optoelectronics Letters, 12(6), 473-477.

Xu, Y., Yu, G., Wang, Y., Wu, X., Ma, Y., 2016. A Hybrid Vehicle Detection Method Based on Viola-Jones and HOG + SVM from UAV Images. Sensors (Basel, Switzerland), 16(8), 1325.

Zhang, X., Cui, J., Wang, W., Lin, C., 2017. A Study for Texture Feature Extraction of High-Resolution Satellite Images Based on a Direction Measure and Gray Level Co-Occurrence Matrix Fusion Algorithm. Sensors (Basel), 17(7). 


\section{Chapter 5 - Post-disaster building database updating using automated deep learning: An integration of pre-disaster OpenStreetMap and multi-temporal satellite data ${ }^{4}$}

\footnotetext{
4 This chapter is based on:
}

- Ghaffarian, S.; Kerle, N.; Pasolli, E.; Jokar Arsanjani, J., 2019. Post-disaster building database updating using automated deep learning: An integration of pre-disaster OpenStreetMap and multi-temporal satellite data. Remote Sens., 11, 2427. 


\section{Abstract}

First responders and recovery planners need accurate and quickly derived information about the status of the buildings as well as the newly built ones to both help victims, and to make decisions for reconstruction processes after a disaster. Deep learning and in particular convolutional neural network (CNN)based approaches have recently become state-of-the-art methods to extract information from remote sensing images, in particular for image-based structural damage assessment. However, they are predominantly based on manually extracted training samples. In the present study we use pre-disaster OpenStreetMap building data to automatically generate training samples to train the proposed deep learning approach after the co-registration of the map and the satellite images. The proposed deep learning framework is based on the U-net design with residual connections, which has been shown as an effective method to increase the efficiency of CNN-based models. The ResUnet is followed by a Conditional Random Field (CRF) implementation to further refine the results. Experimental analysis was carried out on selected very high resolution (VHR) satellite images representing various scenarios after 2013 Super Typhoon Haiyan in both the damage and the recovery phases in Tacloban, the Philippines. The results show the robustness of the proposed ResUnet-CRF framework in updating the building map after a disaster for both damage and recovery situations by producing an overall F1-score of $84.2 \%$. 


\subsection{Introduction}

Post-disaster map updating is one of the essential tasks to support officials/governments to make decisions, policies and plans for both the response phase to conduct emergency actions, and the recovery phase to return to normalcy after the event, and even to build back better as per the Sendai Framework (UNISDR 2015). Buildings constitute an essential land cover class in the affected area. Consequently, updating the building database is vital to provide accurate information related to demolition, reconstruction and building modification taking place during the response and recovery phases (Ghaffarian et al. 2018). Building map updating requires new building data for detecting changes in the status of the buildings and the identification of newly built ones. Satellite remote sensing (RS) has become an essential and rapid tool for acquiring suitable geospatial data given its synoptic coverage and ready availability. In addition, the availability of free high-resolution images that are provided by platforms such as Google Earth has also been attracting researchers in the remote sensing domain to focus on image-based building detection and mapping (Ghaffarian and Ghaffarian 2014a, 2014b).

Building database updating can be done based on two general frameworks: building detection/extraction from mono temporal RS data, and building change detection using multi-temporal RS data (Feng and Zhao 2009; Ghanea et al. 2016; Sofina and Ehlers 2016). The second framework is the focus of this study and usually comprises two main steps: 1 - extracting buildings, and 2 - detecting changes. Change detection approaches can be grouped based on the type of data they use:

1- Multi-temporal RS data: The multi-temporal data are directly considered to detect changes, for example, from multi-temporal satellite images with a pixel-by-pixel or an object-/feature-based change analysis by comparing two images (Liu et al. 2018; Singh 1989).

2- Multi-temporal RS and map data: In this approach, the multi-temporal RS data are classified using additional support from existing maps by providing guidance in training area selection (Knudsen and Olsen 2003b), or excluding non-building pixels based on a probability analysis (Holland et al. 2008; Rottensteiner 2007). Then the maps or the classified building images are compared to detect changes in buildings in an object-oriented manner (Armenakis et al. 2003; Walter 1999).

3- Monocular RS and old map data: In many cases, pre-disaster highresolution RS data of the affected region do not exist, precluding method 1 from being used. However, the old geo-databases containing building information can be used to guide the method to find changes in the building stock (Bouziani et al. 2010; Gharibi et al. 2014; Le Bris and Chehata 2011). This method is more complicated than the previous one 
because it contains a level of generalization and abstraction (Bentabet et al. 2003; Fiset et al. 1998), and existing databases may not accurately reflect the immediate pre-disaster situation. However, the method can provide valuable information about relevant feature classes (Baltsavias 2004).

4- Height-related data: Approaches that use height data such as Digital Surface Models (DSMs), including height information obtained through LIght Detection And Ranging (LiDAR) and Unmanned Aerial Vehicle (UAV) data. Height-related data from DSMs and LiDAR data are generally utilized as changed or non-changed features to detect building changes (Choi et al. 2009; Jung 2004; Tian et al. 2011; Xu et al. 2015).

In this paper we propose a framework to provide an automatic updating of the building database from very high resolution (VHR) satellite images and outdated map data. OpenStreetMap (OSM) data were selected to be used as the reference building layer, due to their free availability. OSM provides global coverage of crowdsourced geoinformation and has become the premier Volunteered Geographic Information (VGI)-derived cartographic database, though with spatially varying data quality. OSM has been proven to be even more accurate than proprietary data in some areas (e.g., generally in Europe) (Zielstra and Zipf 11-14 May 2010), while not offering the same accuracy or completeness in many more remote parts of the world (Brovelli and Zamboni 2018; Siebritz and Sithole 1-3 July, 2014). Since a wide range of volunteers, including highly-experienced and amateurs, contributes to the OSM data collection, certain limitations apply when dealing with OSM data (Jokar Arsanjani et al. 2015). OSM building datasets contain a number of errors: a) omission errors, which indicate actual buildings not mapped in OSM, b) spatial mismatch/shift of the building footprints compared to satellite images, c) geometric errors, which indicate that the size and/or shape of the actual buildings do not match with the mapped ones, and d) thematic information/label errors, which indicates that the information regarding the types/use of the buildings does not match the actual building use/information. In addition, e) mismatch of the building rooftop with the footprints can occur in satellite images due to the incident angle of the sensor during their acquisition.

Since the present study is dedicated to automatic building database updating from multi-temporal VHR satellite images and (potentially outdated) OSM map data, we primarily discuss the previous studies that used RS datasets guided by old map data to generate new building maps.

Fiset et al. (1998) introduced the use of maps to guide a change detection algorithm to update road network maps. Afterwards Bentabet et al. (2003) proposed a new approach to refine and update road vector data using SAR 
images. Regarding building change detection using existing map data, Knudsen and Olsen (2003a) developed a method that combines supervised and unsupervised comparisons and tested their method to update Denmark's map database. They used a conventional classification method to extract buildings, which produced a high amount of false positives in detecting buildings. Bouziani et al. (2010) proposed a heuristic technique based on contextual information obtained from old map data. Their method was based on objectbased image analysis (OBIA) classification and fuzzy logic-based rules to extract the changed buildings from VHR images. However, the inclusion of rules based on color, size, and spectral information results in the accuracy being strongly correlated with the possibility of tuning the OBIA parameters properly. Building change detection was done in (Matikainen et al. 2010) by first segmenting DSM information produced from laser data. Then, aerial images and laser data were used to obtain a classification map, which was further refined through information from an old map. Results were promising and exhibited accuracy values in terms of completeness and correctness of about $85 \%$ for buildings larger than $60 \mathrm{~m}^{2}$. However, their method is highly dependent on the quality of the DSM data, which are critical for the removal of non-building features. Le Bris and Chehata (2011) conducted a comparison analysis including methods that rely on images and old maps, and strategies based on multi-temporal images for building map updating. They concluded that such methods were not appropriate for operational uses. Similar approaches were investigated by Malpica et al. (2012) and Gharibi et al. (2014), who used old map data and a LiDAR-based nDSM to guide a Support Vector Machine (SVM) classification and Level Set method for building change detection. Although their methods provided good results, nDSM data were again critical for building detection. This departs from what we propose in the present paper, in which only multispectral VHR satellite images are used, therefore without considering any products that include height information. Furthermore, most of the previous studies did not consider the building change in different scenarios, particularly in a disaster situation. For example, a preevent building may either be damaged and rebuilt or demolished, change into a different shape or type during recovery, or be rebuilt in a new place.

Concurrent with the development of map-guided building change detection methods, computer vision and RS data processing methods have evolved, and based on recent advances in computer hardware systems researchers can readily run deep neural network-based models. Deep learning and convolutional neural networks (CNN) have been investigated and become the state-of-the-art for many computer vision tasks. These methods have been also used for RS data processing problems such as scene classification (Yang et al. 2015; Zhao et al. 2016), hyperspectral image classification ( $\mathrm{Li}$ et al. 2017b; Mou et al. 2017a, 2017b), object detection (Cheng et al. 2016; Jin and Davis 2007), image retrieval (Jiang et al. 2017), multi-modal data fusion 
(Duarte et al. 2018a; Loncan et al. 2015), and change detection (Li et al. 2017a; Lyu et al. 2016; Zhang et al. 2018b). However, the developed deep learning-based change detection methods aim at detecting scene-based changes rather than a specific object and need further processes to be used in RS applications. Deep learning, in particular CNN, has also been used for disaster-based applications, such as structural damage assessment (Duarte et al. 2018a; Duarte et al. 2018b, 2019; Nex et al. 2019; Saha et al. 2018; Vetrivel et al. 2017), as well as landslide (Chen et al. 2018; Xiao et al. 2018) and fire detection (Zhao et al. 2018). Most of the developed methods for building damage assessments require VHR UAV images and/or 3D point clouds, and aim at assessing only structural damages. However, changes in the status of the buildings months and years after a disaster provide crucial information for post-disaster recovery assessment, which is addressed in the present paper. In a recent study, Ji et al. (2019) proposed a method to detect collapsed buildings after an earthquake using pre- and post-disaster satellite imagery. Their method made use of CNN-based features to be used in a random forest classifier to do the detection work. Although the method was able to extract collapsed buildings with high accuracy, the building map was manually generated, a subjective and time-consuming task. This problem is overcome by our fully-automated methodology based on the OSM building map. Furthermore, their method was aimed at detecting collapsed building after an earthquake, which is inherently unsuitable for detecting new buildings or changes in building size or shapes during the recovery/reconstruction phase as exploited in our current work.

In the present paper we adapted the deep residual U-net (ResUnet) developed by (Zhang et al. 2018a) as the classifier, and the training area was selected automatically using the pre-disaster building OSM data after a preprocessing step that co-registered the OSM and satellite data. The network was first trained on the pre-disaster OSM data and pre-disaster satellite images, and then fine-tuned using the same building map after conducting a building-based change detection process from a post-disaster satellite image. The change detection step was done based on two textural measurements to select appropriate training areas. Two measures, i.e., Variation-Histogram of the Oriented Gradients (V-HOG) and Edge Density Index (EDI), were considered to perform the change detection. This step was essential to exclude those buildings that may have been destroyed or damaged during the disaster from the training set when retraining the network for the period just after disaster. Furthermore, by fine-tuning the pre-trained network on the post-disaster images after conducting the change detection step, the proposed method was able to detect buildings in the post-disaster (recovery) time that were newly constructed, and to extract changes in the size or shapes of the existing buildings. As final step, Conditional Random Field (CRF) was performed to refine the boundaries and improve the classification results, similar to the 
methods investigated in (Alam et al. 2018; Pan and Zhao 2018). The proposed framework was evaluated using WorldView2 satellite images of Tacloban, the Philippines, which was hit by Typhoon Haiyan (Yolanda) in 2013. Images were acquired one month before the disaster, three days, and four years after the disaster.

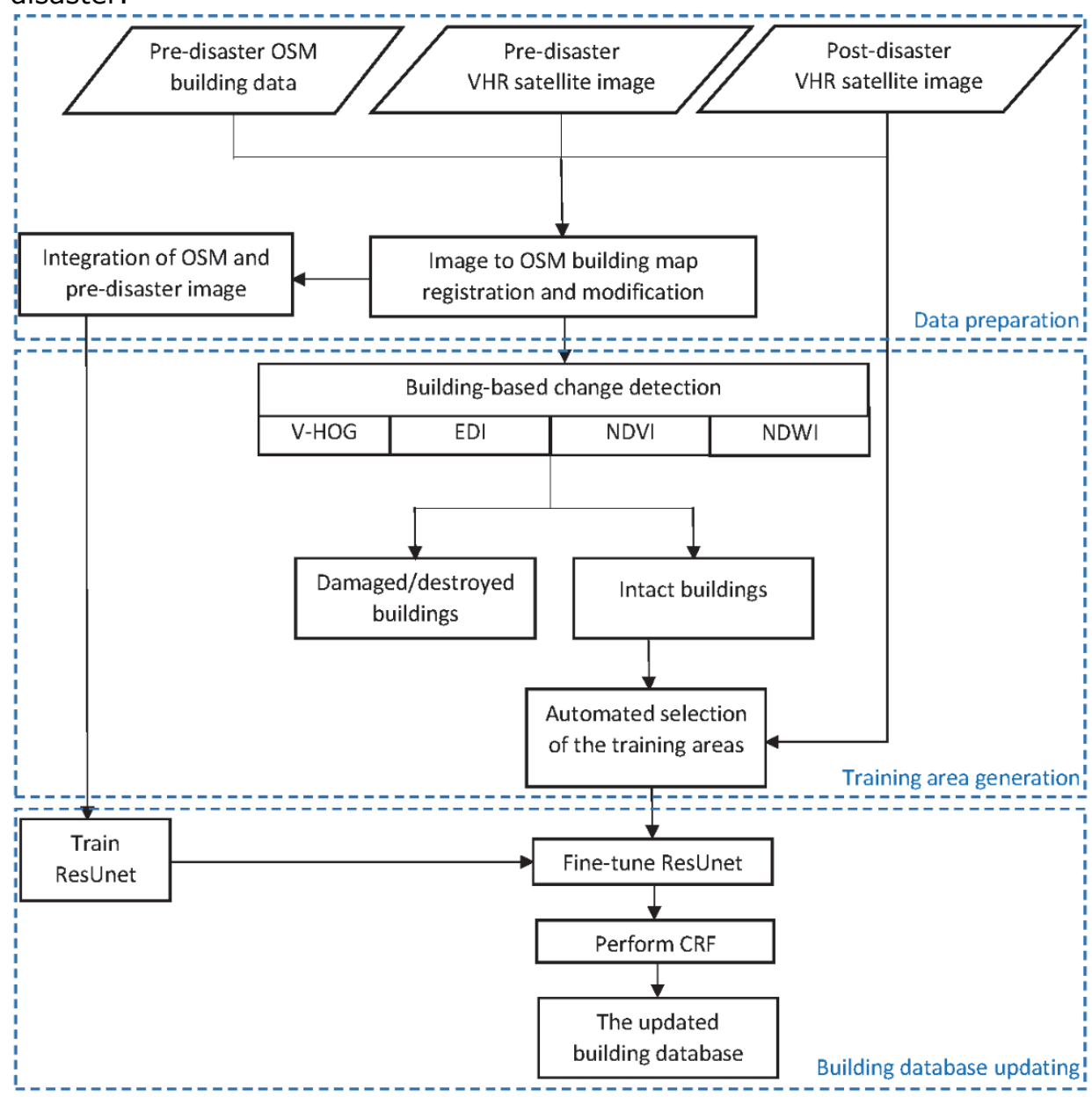

Figure 5.1 The framework proposed in this paper for post-disaster building database updating. Notes: V-HOG = Variation of Histogram of Oriented Gradients; EDI = Edge Density Index; $\mathrm{CRF}=$ Conditional Random Field.

\subsection{Materials and Methods}

In this paper we propose a framework for updating the building database after a disaster through an automated ResUnet-CRF, using outdated OSM building data and multi-temporal satellite images (Figure 5.1). The proposed approach consists of four main steps. 


\subsubsection{Step 1: Co-registration of OSM data and satellite images}

Since the aim of this study was to use OSM building data as a mask to extract building training samples from the pre-disaster image for a CNN-based approach, we implemented simple preprocesses to create accurate training samples. Since it was observed that the shift on the building footprints is not systematic and the shift direction and amount differs substantially across the area, initially the downloaded OSM data for the pre-disaster time were separated into five sections/regions. Then rubber sheeting was implemented in ArcGIS, which can also handle small geometric correction as well as shifting the vector maps, to adjust and align the building map to the building rooftops in the pre-disaster image (Figure 5.2). In order to achieve good results from the application of the rubber sheeting method, five geographically welldistributed points within each region of interest were used, so that the points cover at least the center and the four main directions. Furthermore, the postdisaster satellite images were co-registered/rectified according to the predisaster image using ArcGIS by selecting geo-rectification points.
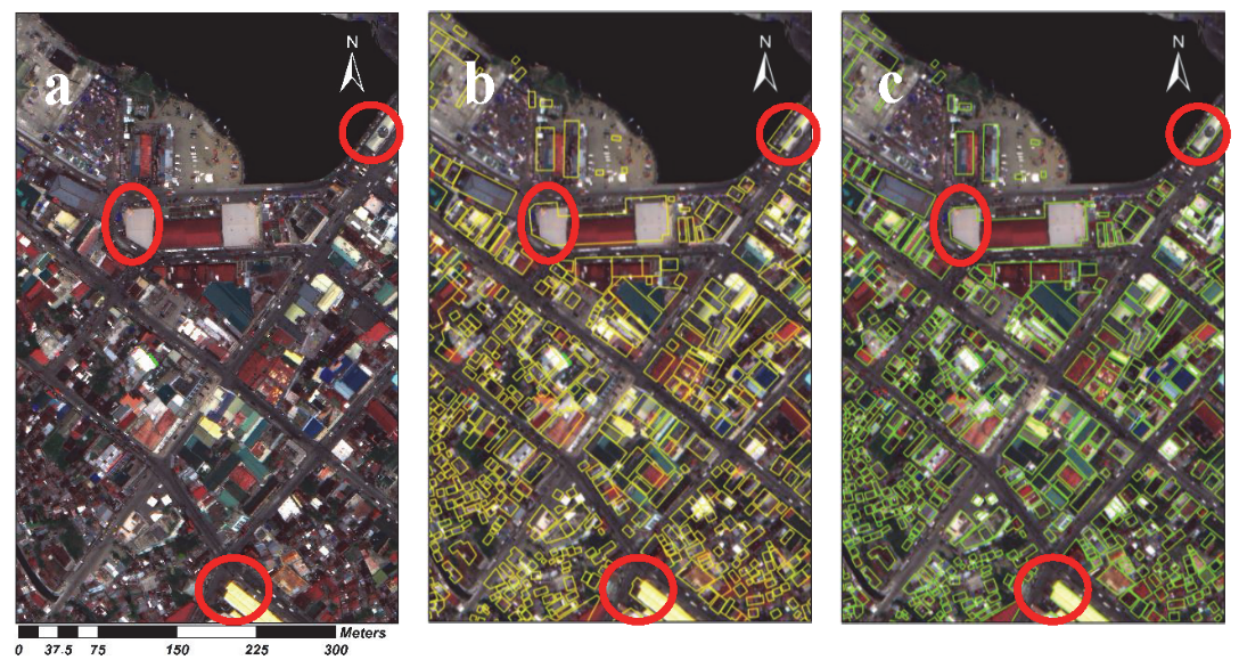

Figure 5.2 Example of the co-registration of the OSM building map and satellite images for Tacloban city, the Philippines. (a) Pre-disaster satellite image, (b) original OSM building map, and (c) modified OSM building map. The areas denoted by red boundaries show the effect of the refinements on OSM map data.

\subsubsection{Step 2: Training patch generation from the pre-disaster image}

Pre-processed data from step 1 were used to automatically generate training samples from the pre-disaster image. Although the mismatch between OSM building footprints and the actual buildings in the pre-disaster images was 
mostly corrected for in step 1, some matching errors remained. For example, in the case that a building near a vegetated area has a mismatch, the building mask might contain vegetation pixels. In addition, even in a correct match of building and OSM map some non-building pixels might end up inside the training samples e.g., a tree may partially cover a rooftop of a building. This case might also occur where the buildings are next to the sea/water bodies, which may lead to the inclusion of water pixels in the training samples. Hence, to overcome these issues the Normalized Difference Vegetation Index (NDVI) and Normalized Difference Water Index (NDWI) indices were computed for the pre-disaster image to exclude vegetated areas, trees, and water bodies from the building mask. To do so, NDVI and NDWI masks were computed based on pre-defined thresholds and those pixels falling into the masks were removed from the building training class. Training patches with a height and width of 512 pixels were extracted from the entire image. Moreover, in order to increase the training samples, more patches were generated from the same area by shifting the starting point of the generation of the patches by 100 pixels in both $x$ and $y$ directions. This procedure was conducted three times to obtain different image patches from the same area, and then the suitable training samples were selected from those to train the network. In total, 780 image patches were selected to be used as initial training sample set. In addition, 542 image patches were selected for each of disaster and post-disaster images to be used for fine-tuning of the model.

\subsubsection{Step 3: Detecting damaged and demolished buildings}

The OSM building map represents the pre-disaster time; however, since some buildings get damaged during the disaster and are later demolished, direct use of those maps for the training area selection from post-disaster images will lead to inaccurate results, as rubble and damaged buildings are included. Hence, the damaged and destroyed buildings should be excluded from the building footprint map before using them for training area selection for postdisaster time images. Since the extraction of the buildings will be based on the advanced proposed deep learning approach, a simple yet accurate method was developed only for the identification of the intact and damaged buildings from the provided OSM building map.

Two measurements based on the Histogram of the Oriented Gradients (HOG) and the edge detection results of the satellite images, namely Variation-HOG (V-HOG) and Edge Density Index (EDI), were used to conduct the change detection between the pre-disaster and post-disaster satellite images. The change detection was performed only on the building masks of the images to distinguish the damaged/demolished and intact buildings in the post-disaster image. 


\subsubsection{Variation of HOG (V-HOG)}

HOGs provide powerful features (Dalal and Triggs 2005) for image representation, which are particularly robust for image-based object classification. They were initially developed for pedestrian identification (Geronimo et al. 2010), but were then found to be robust features in different applications (Geronimo et al. 2010), including for remote sensing data (Cheng et al. 2016; Patel et al. 2016; Torrione et al. 2014; Xu et al. 2016) and for image-based damage detection (Vetrivel et al. 2016).

The standard approach was used to extract the HOG features, which starts by computing the gradient angles of the image and their magnitude distributions. Then the images were split into cells of size $(a \times b)$. Gradient images were split into overlapping blocks in a manner that each block contained $50 \%$ overlap with the cells. Then the orientation of the gradients was computed based on the defined bin size. The histogram of the oriented gradients was computed as a vector and concatenated for each block after adding the normalized magnitude of the gradients. Since damaged areas have a larger HOG distribution compared to intact buildings, we considered the V-HOG to compute the variation of the normalized magnitude of the gradients of the bins to detect damaged buildings (Figure 5.3e). Hence, a higher variation of the HOG descriptor (higher V-HOG value) represented damaged areas, while small VHOG values indicated intact buildings. The V-HOG can be computed for each block or each pixel, similarly to the HOG. However, HOG features may show high variation in some cases due to color differences between pixel values in building roofs. For example, a building may contain more than one color in its rooftop, which was overcome by conducting a building-based change analysis, rather than considering only the mono temporal image. This rasterized value can be used simply by defining a threshold in the change in mean of V-HOG from pre- to the post-disaster image to distinguish damaged/demolished buildings from the intact ones. 

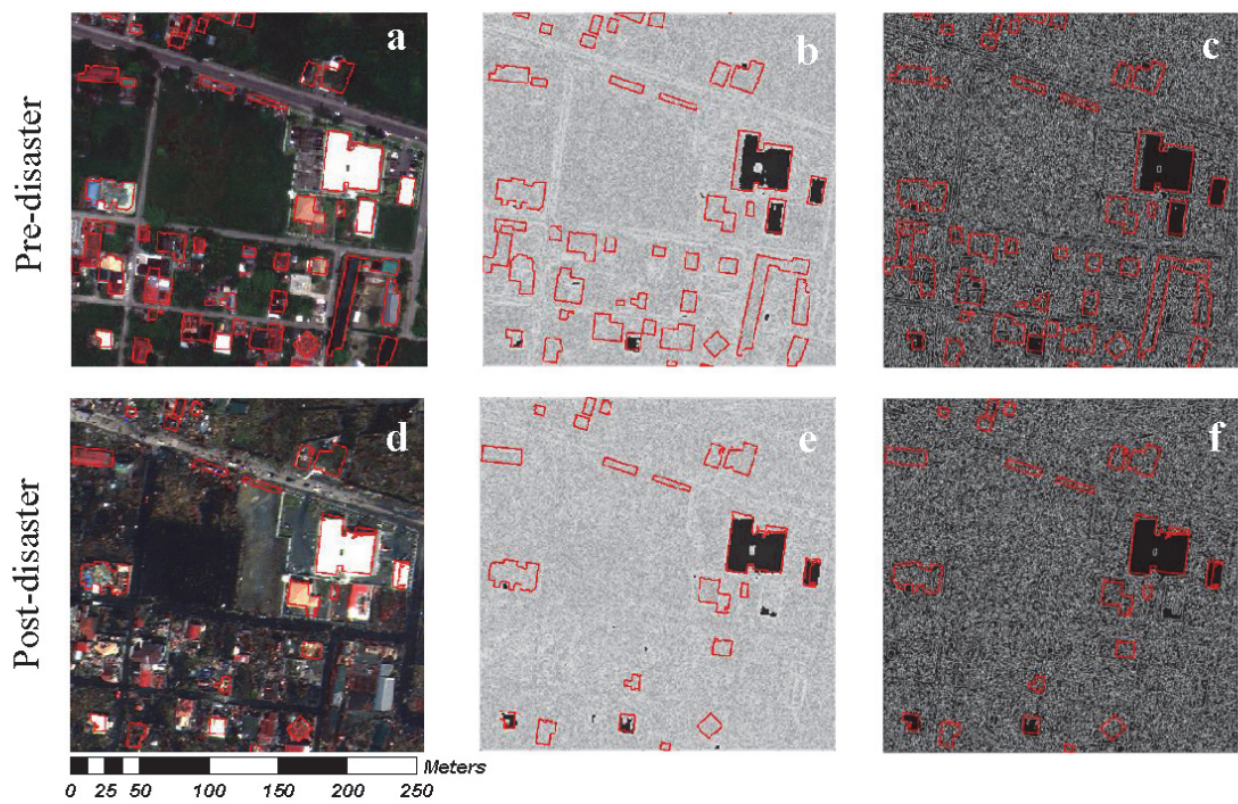

Figure 5.3 (a-c) Pre-disaster data in terms of (a) multispectral image, (b) V-HOG and (c) edge detection image; OSM building map denoted with red lines. (d-f) Event time data in terms of (d) multispectral image, (e) V-HOG and (f) edge detection image; . result of Step 3 denoted with red lines.

\subsubsection{Edge Density Index (EDI)}

Edge detection results have previously been employed to refine image classification (Marmanis et al. 2018) and object boundary detection (Ghaffarian and Turker 2018). In our study we used the edge detection results to detect changes in building status, i.e. to differentiate among damaged, demolished and intact buildings. Since damaged buildings are expected to contain greater variations in their pixel values inside the building footprints due to the presence of debris/rubble, they were expected to contain more edge pixels when compared to intact buildings that had more stable color variation. Accordingly, the number of edge pixels along a building damaged during a disaster was higher than that of buildings in the pre-disaster situation that were not damaged (Figure 5.3c,f). Since the size of a building could vary from large factories to very small slum dwellings, the number of edge pixels should be considered based on the corresponding building size. Hence, Edge Density Index (EDI) was proposed, which measures the percentage of edge pixels within a building area, and if the change was higher than the defined threshold the building was considered to be damaged/demolished. Edges were detected using the Canny edge detector (Canny 1986), and its two parameters were set to extract even weak edge pixels from the images. 
After a disaster and during the reconstruction process, the rooftop color of a building may change, and thus conventional techniques that perform direct change detection such as pixel value-based subtraction methods (Celik 2009; Lu et al. 2004) were not suitable for this aim. However, the two proposed indices were not sensitive to the changes in the rooftop colors of buildings from the pre- to the post-disaster scenario. In addition, since the change detection is at building level and is followed by an advanced deep learning approach, a simple yet accurate method is required rather than more complicated methods that include contextual information (Janalipour and Mohammadzadeh 2016). The buildings in each image patch were considered individually, and each building was taken into account at each time. Subsequently, the intact buildings were extracted. Furthermore, NDVI and NDWI were used to remove vegetated areas and water bodies and to refine the results from the building mask in the post-disaster image. Only pre-disaster OSM building data were used for the damage analysis (Figure 5.3a), and thus the damaged buildings (in the event time case) and demolished buildings (in the recovery case) were detected based on changes in the mean V-HOG and EDI.

\subsubsection{Step 4: Updating the building database}

The output of step 3 is affected by three main problems: 1 ) buildings that are present in the images but are missing in the OSM building map data cannot be detected by the procedure implemented in step $3 ; 2$ ) inaccuracies may occur especially due to mismatches and inaccuracies of OSM building map data. This, for example, will classify an intact building as damaged due to connected and adjacent buildings; and 3 ) it gives only changes of buildings existing before the disaster, therefore missing the capability to extract newly constructed buildings. To overcome these relevant issues, step 3 is followed by step 4 which is based on an automated deep learning-based network as we will detail later. Furthermore, since the method is a pixel-level classification it can extract the shape and size of the buildings and thus their changes. The method is primarily based on the adapted deep residual U-net (Zhang et al. 2018a) to automatically train and detect buildings for post-disaster situations. U-net has been shown to be reliable for image segmentation tasks (Bai et al. 2018; Yuan et al. 2019; Zhu et al. 2017), and residual connection has been also demonstrated as one of the effective network designs to detect building damages (Duarte et al. 2018a). Figure 5.4 shows the DeepResUnet-CRF design used in our study. We used only historical OSM data for the initial training of the network from the pre-disaster image, in which there were inaccuracies even after refinements of the OSM building maps. Therefore, only suitable ones were selected to train the network. In addition, transfer learning has been shown to be an effective method to accelerate the training process and increase the accuracy performance of the network in several computer vision (Gopalakrishnan et al. 2017; Ker et al. 2018; Shin et al. 2016) and remote 
sensing applications (Huang et al. 2017; Li et al. 2017c; Zhu et al. 2017). Hence, the Resnet34 trained network from ImageNet was considered as pretrained network. Given that every satellite image may differ from the other ones in terms of image characteristics (e.g., radiometric range values) and changes in building properties (e.g., colors) after a disaster, the network trained on the pre-disaster situation/images may not provide accurate results for the post-disaster situations/images. Hence, the results of step 3 were used to generate new samples from the associated post-disaster satellite images to fine-tune the pre-trained network. The fully connected networks and U-net have a common limitation in image segmentation tasks, which is the smoothing of edges. In addition, since the OSM building map did not provide a precise building mask, particularly for the building boundaries, we verified inaccuracies in some parts of the images. This problem was alleviated by implementing a Conditional Random Field method (CRF), which has been primarily investigated in the literature as a refinement over the U-net or Fully Connected Networks (FCNs) results (Liu et al. 2019; Pan and Zhao 2018; Wang et al. 2018). Accordingly, a fully/dense CRF model developed by Krähenbühl and Koltun (2011) was employed to optimize the ResUnet results.

Labels for each pixel can be considered as random variables, and their relations in the image can be considered as edges in a graph-based theory, and these two factors constitute a conditional random field. In dense CRF two main factors in its energy function are the unary and pairwise potentials.

Let $x$ be the pixel-level labels for the input image, then the unary potential $\varphi_{i}\left(x_{i}\right)$ represents the probability of each $i$ pixel, and the pairwise potential $\tau_{i, j}\left(x_{i}, x_{j}\right)$ that represents the cost between labels at $i, j$ pixels is computed as follow:

$$
\tau_{i, j}\left(x_{i}, x_{j}\right)=\mu\left(x_{i}, x_{j}\right)\left[\omega_{1} \exp \left(-\frac{\left|l_{i}-l_{j}\right|^{2}}{2 \theta_{\alpha}^{2}}-\frac{\left|c_{i}-C_{j}\right|^{2}}{2 \theta_{\beta}^{2}}\right)+\omega_{2} \exp \left(-\frac{\left|l_{i}-l_{j}\right|^{2}}{2 \theta_{\gamma}^{2}}\right)\right]
$$

where $l_{i}$ and $C_{i}$ are the position and color vector for pixel $i . \mu\left(x_{i}, x_{j}\right)$ is defined based on the Potts model (Potts 1952) and is equal to one if $x_{i} \neq x_{j}$, otherwise it is equal to zero. The first Gaussian expression considers both color and location of the pixels, which is an appearance kernel to consider the similarity of the adjacent pixels using the $\theta_{\alpha}$ and $\theta_{\beta}$ parameters, and the second expression only consider pixel positions and is for smoothness using the $\theta_{\gamma}$ as the control parameter.

Then the energy function can be written as follow:

$$
E(x)=\sum_{i} \varphi_{i}\left(x_{i}\right)+\sum_{i j} \tau_{i j}\left(x_{i}, x_{j}\right)
$$

The CRF is an iterative method that evolves and computes the labels and predictions. 


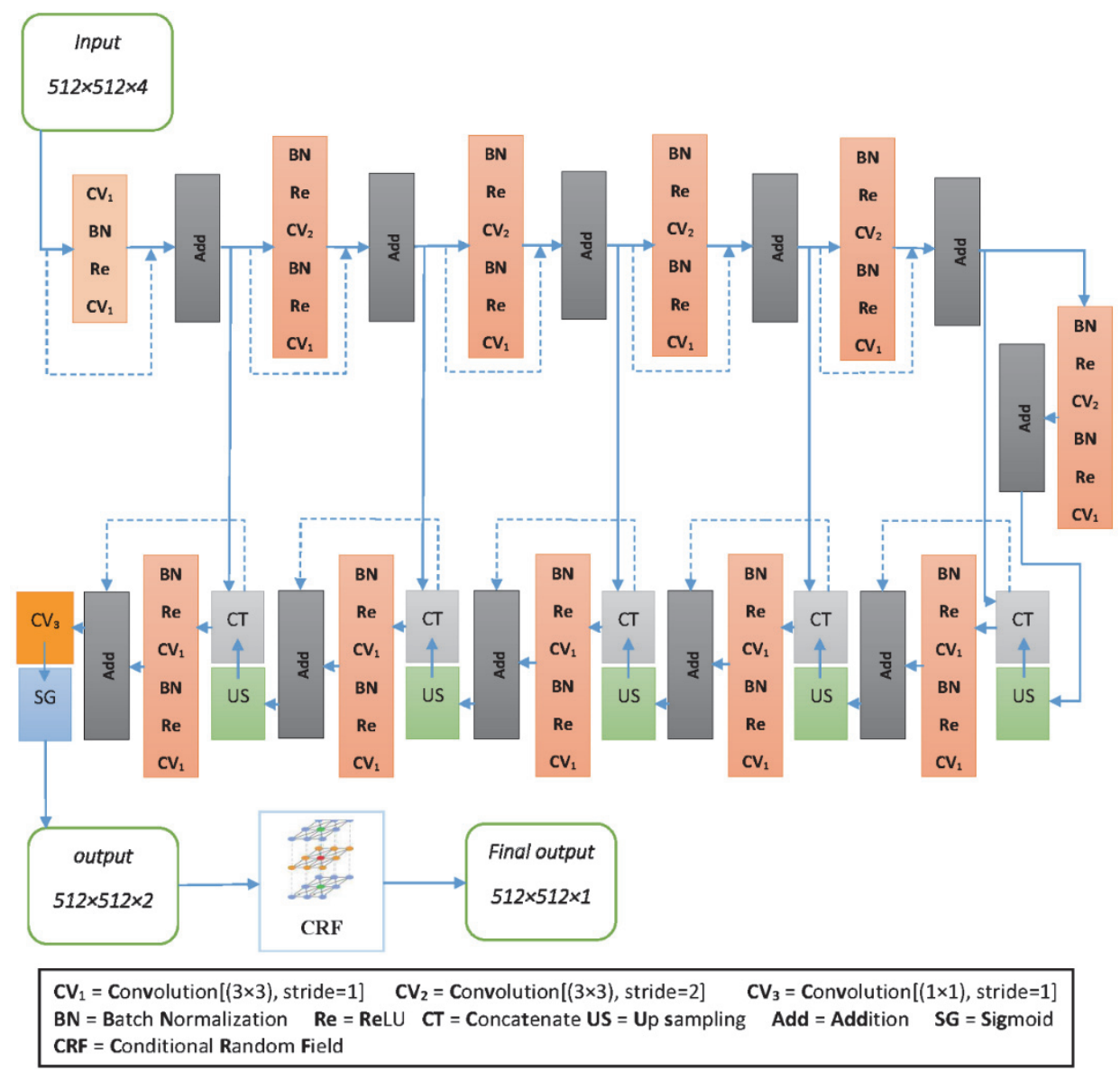

Figure 5.4 The proposed ResUnet-CRF framework.

\subsection{Experimental results}

\subsubsection{Datasets}

We tested the proposed post-disaster building database updating framework on satellite images of Tacloban city, the Philippines, which was hit by super Typhoon Haiyan in November 2013, resulting in massive damages and losses (Figure 5.5). Tacloban is a highly urbanized city that is extensively vegetated due its tropical location. There are several types of built-up regions in the city, including dense urban areas mostly located in the central business district of the city with adjacent buildings, slum areas, mix of slum and formal buildings, isolated buildings surrounded by dense vegetation/trees, various building shapes and sizes from very small slum dwellings to large factories, and diverse rooftop colors. All these characteristics made the building detection procedure challenging and a suitable test area for our method. 


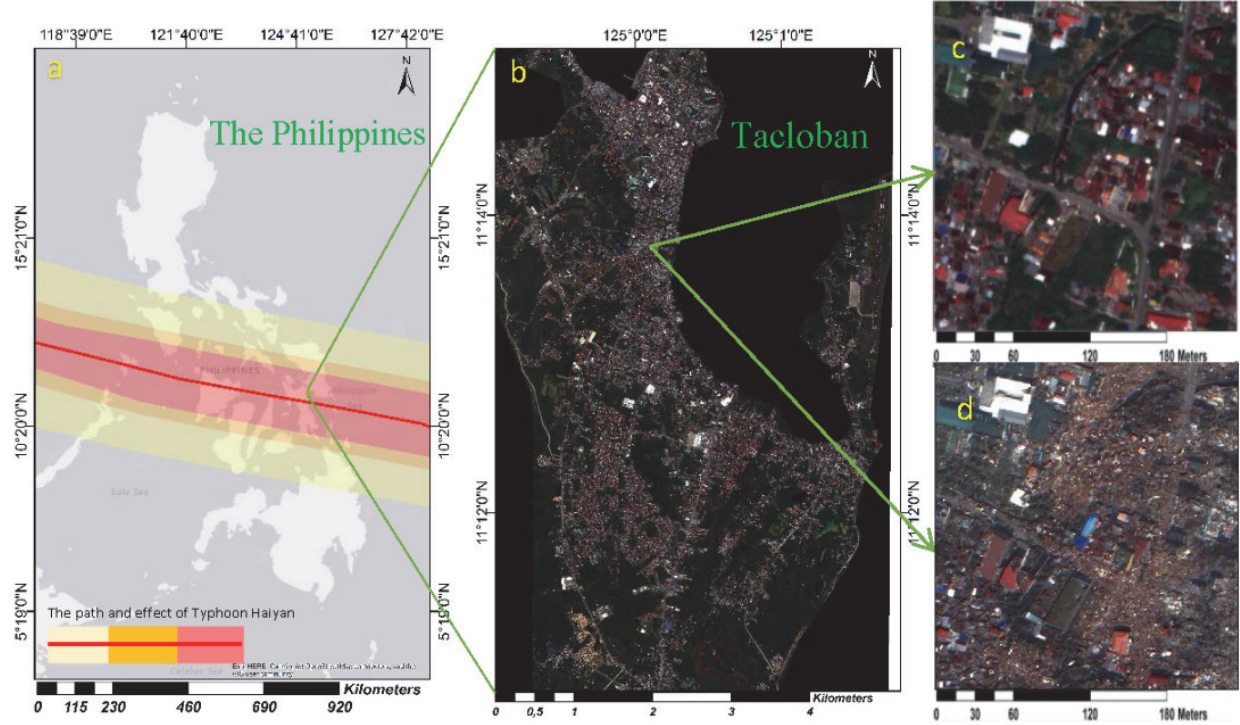

Figure 5.5 An overview of the Philippines showing the path of Typhoon Haiyan (a) and the location of Tacloban city (b). Pre-disaster image (c), and image acquired three days after the disaster (d).

The WorldView2 (WV2) pan-sharpened images with $0.5 \mathrm{~m}$ spatial resolution and four multispectral bands (Blue, Green, Red, NIR) acquired 8 months before, 3 days after and 4 years after the Typhoon were used in the work. The selection of 4 bands (Red, Green, Blue, and NIR) instead of using entire 8 bands available in the satellite images is to reduce the computational complexity/time of the processes while using the most informative bands of the satellite images for our goal. In addition, OSM (historical) building data for 2013 obtained from the OSM platform were used as the pre-disaster building map data.

\subsubsection{Experimental settings}

The proposed method was applied to ten selected image patches to evaluate its capabilities on urban areas characterized by various building and environmental characteristics. The test images were not included in the training process of the network, and were specifically selected to test the performance of the proposed approach in various environmental/data setsbased conditions, as well as different damage and recovery (reconstruction) scenarios (Table 5.1). 
Table 5.1 The targeted post-disaster building detection scenarios for each selected test images.

\begin{tabular}{|c|c|}
\hline Image & Targeted post-disaster building detection scenarios \\
\hline$\# 1$ & Buildings that survived the disaster \\
\hline$\# 2$ & Partially destroyed slums and formal buildings \\
\hline$\# 3$ & Buildings surrounded by flood water \\
\hline$\# 4$ & $\begin{array}{l}\text { Completely destroyed slums that produced an extensive amount of } \\
\text { debris }\end{array}$ \\
\hline$\# 5$ & Partially damaged factory buildings \\
\hline$\# 6$ & $\begin{array}{l}\text { Reconstructed and not-reconstructed (completely cleared/removed) } \\
\text { buildings after } 4 \text { years }\end{array}$ \\
\hline \#7 & $\begin{array}{l}\text { Reconstruction of the buildings almost to the same amount, shape } \\
\text { and sizes }\end{array}$ \\
\hline$\# 8$ & $\begin{array}{l}\text { Construction of new buildings and changes in rooftop colors in the } \\
\text { recovery phase }\end{array}$ \\
\hline$\# 9$ & $\begin{array}{l}\text { Clear expansion of the built-up area and construction of new } \\
\text { buildings }\end{array}$ \\
\hline$\# 10$ & Change in the size of the reconstructed factory building \\
\hline
\end{tabular}

Table 5.2 presents the parameters and thresholds which were employed in the implementation of the developed method. The results obtained by the proposed automatic procedure were compared with reference data produced manually by a qualified human operator. Accuracies were assessed using common precision, recall, $F_{1}$-score, and Intersection over Union (IoU) (Ghaffarian and Ghaffarian 2014a) measurements, all computed at pixel-level. Therefore, initially all the pixels in the image were sorted into four classes: True Positive (TP), True Negative (TN), False Positive (FP) and False Negative (FN). TP and TN show the correct detections, while FP and FN show incorrect detection results. Accordingly, the abovementioned quality measurements can be computed as follow:

$$
\begin{gathered}
\text { precision }=\frac{|T P|}{|T P|+|F P|} \\
\text { recall }=\frac{|T P|}{|T P|+|F N|} \\
F_{1}=\frac{2 \times \text { precision } \times \text { recall }}{\text { precision }+ \text { recall }} \\
I o U=\frac{|T P|}{|T P|+|F P|+|F N|}
\end{gathered}
$$

where $|$.$| denotes the number of pixels assigned to each distinct class, and F_{1}$ score is the combination of precision and recall into a single score. 
Table 5.2 The parameters and threshold values used to do the experiments.

\section{The parameters}

S

\begin{tabular}{|c|c|c|}
\hline o & Edge Density Index (EDI): & \\
\hline ○ & Difference between EDIs for change detection & 0.03 \\
\hline \multirow[t]{3}{*}{ ○ } & Edge detection: Canny & \\
\hline & o Low threshold & 10 \\
\hline & ○ High threshold & 25 \\
\hline O & Variation-HOG (V-HOG): & \\
\hline 0 & $\begin{array}{l}\text { Difference between the mean of V-HOGs for } \\
\text { change detection }\end{array}$ & 0.008 \\
\hline \multirow[t]{4}{*}{ ○ } & HOG: & \\
\hline & ○ Cell size & 2 \\
\hline & ○ Block Size & 1 \\
\hline & o Number of bins & 9 \\
\hline \multirow[t]{4}{*}{ ○ } & Conditional Random Field (CRF): & \\
\hline & $\circ \boldsymbol{\theta}_{\boldsymbol{\alpha}}$ & 35 \\
\hline & $\circ \boldsymbol{\theta}_{\boldsymbol{\beta}}$ & 8 \\
\hline & $\circ \boldsymbol{\theta}_{\boldsymbol{\gamma}}$ & 5 \\
\hline
\end{tabular}

The accuracy values of the proposed approach in extracting buildings from the selected image patches, which are representative of different scenarios, shows the performance of the method in such challenges and conditions.

\subsection{Experimental results and discussion}

The implementation of the Deep ResUnet was carried out on the LISA platform of the SURFSara Dutch supercomputer. This platform is widely available for academic organizations. The pre-processing of the OSM data and the image rectifications were conducted in ArcGIS.

Figure 5.6 shows the automated post-disaster building detection results for the 10 selected images. From those, 5 images were selected from the satellite image acquired 3 days and the other 5 images acquired 4 years after Typhoon Haiyan to test the performance of the proposed method in both the response (damage) and recovery (reconstruction) phases. The TP, FP and FN pixels are illustrated and overlaid on the original images by assigning green, red and blue colors, respectively. In addition, the pre-disaster OSM building map overlaid (yellow color) on the pre-disaster satellite images is shown in the first column of Figure 5.6 to illustrate the changes after the disaster. 

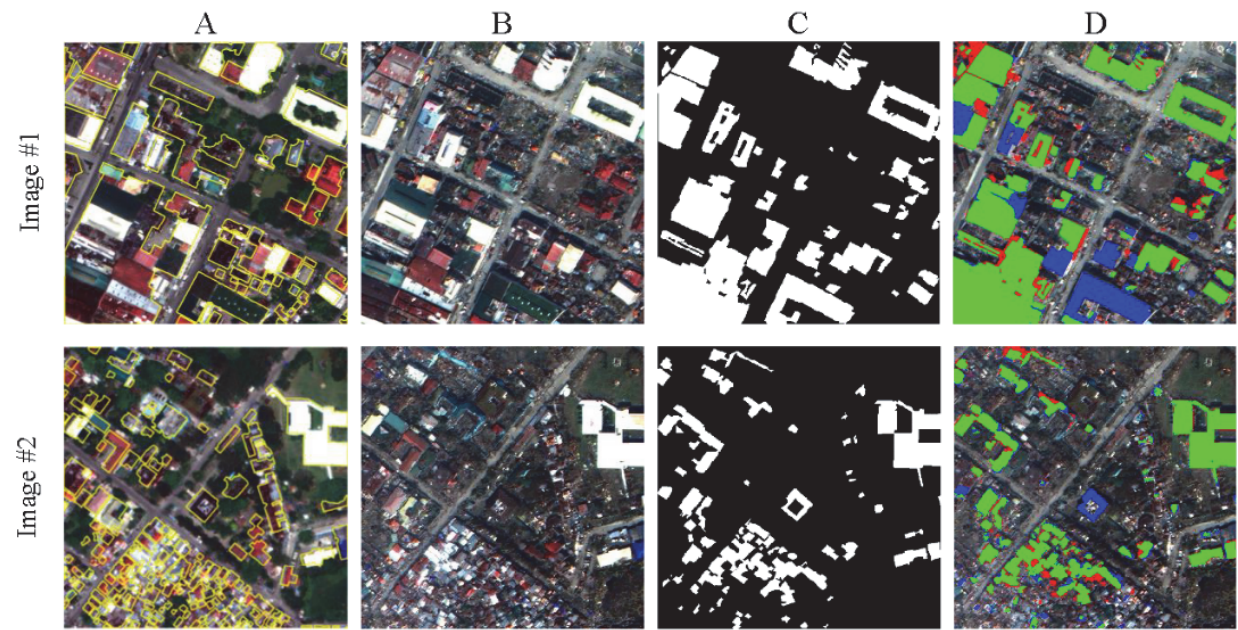

क
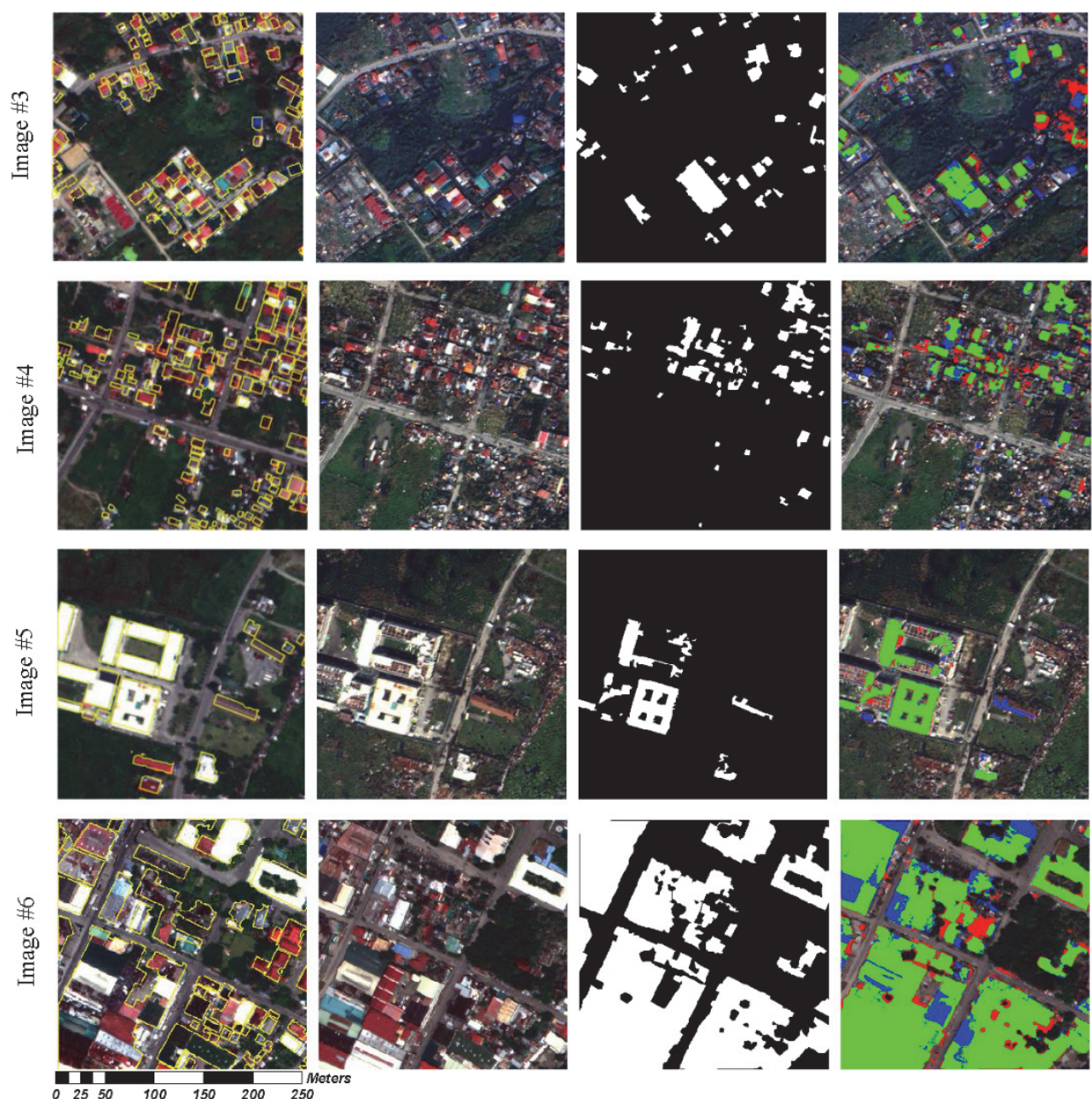

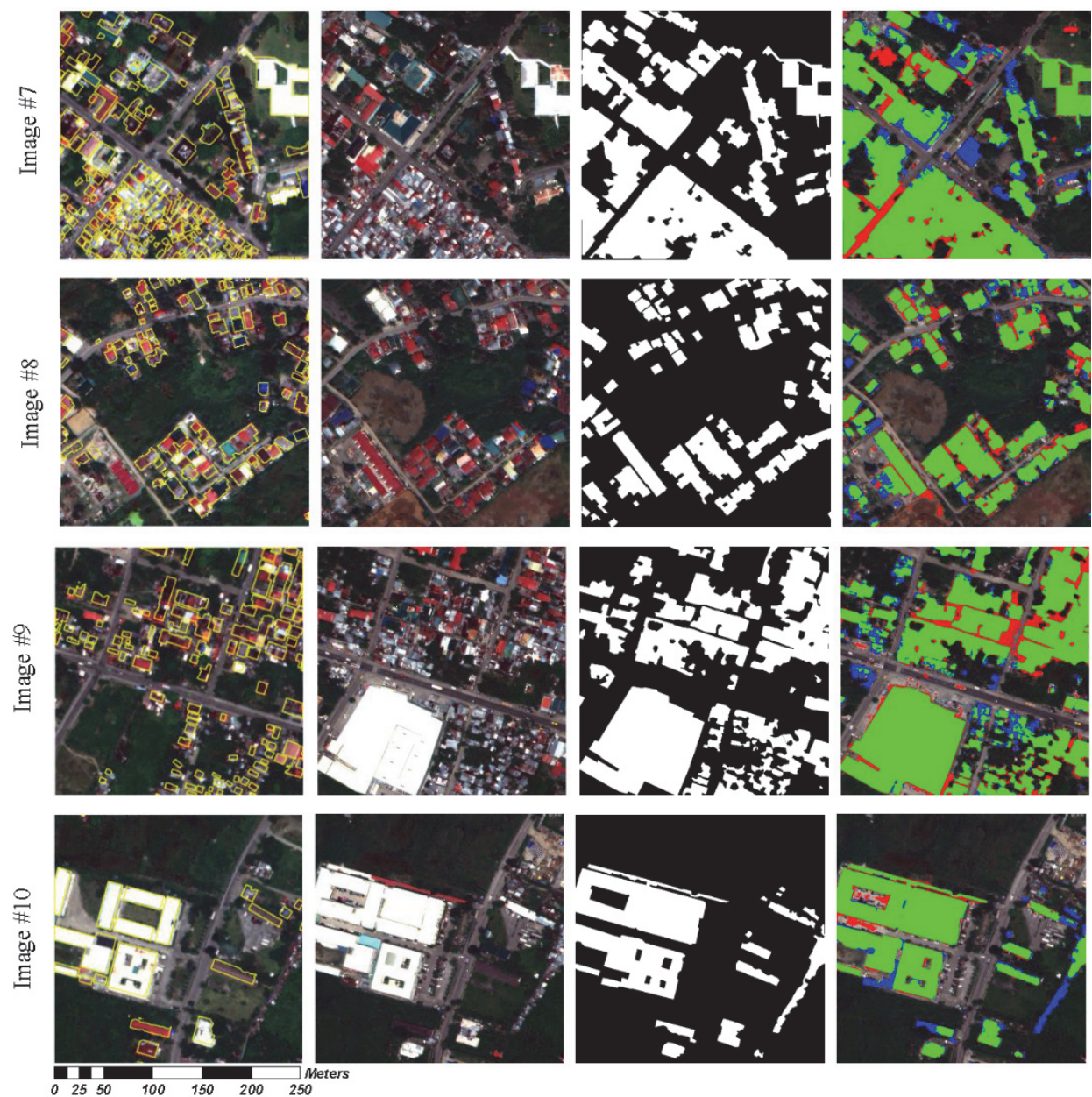

Figure 5.6 The results of the proposed method, test images and pre-disaster images with OSM building boundaries (yellow). Column A: Pre-disaster ( 8 months before Haiyan) images with OSM building boundaries in yellow. Column B, Images \#1-5 taken 3 days after Haiyan and Images\#6-10 taken 4 years after Haiyan. Column C: the reference image for buildings, in which white and black colors represent the building and background pixels, respectively. Column D: detected buildings for test images. Green, red and blue represent TP, FP and FN, respectively.

Qualitative analysis of the results based on the visual interpretation showed the robustness of the proposed method in the extraction of the post-disaster buildings in such a challenging case study by producing more TPs (i.e., green areas), while limiting the FPs (i.e., red areas). Furthermore, the quantitative assessment of the results also supported this statement. The overall $F_{1}$-score for the event-time and recovery time images was $84.2 \%$, the overall precision $84.1 \%$, and the overall recall $84.4 \%$ (Table 5.3 ). The balance between these accuracy measurements also showed the efficiency of the proposed method. In addition, the overall IoU of $73.1 \%$ for such a challenging test area 
demonstrates the performance of the proposed method in extracting building boundaries and their overlap with actual building footprints.

Table 5.3 Numerical results of the proposed post-disaster building database update for event and recovery times.

\begin{tabular}{ccccc}
\hline \multicolumn{5}{c}{ Event time satellite images } \\
\hline & Precision (\%) & Recall (\%) & $F_{1}$ score (\%) & IoU (\%) \\
\hline \#1 & 86.3 & 78.2 & 82.1 & 69.6 \\
\hline \#3 & 84.8 & 84.1 & 84.5 & 73.1 \\
\hline \#4 & 70.6 & 77.0 & 73.7 & 58.4 \\
\hline \#5 & 75.2 & 77.7 & 76.4 & 61.8 \\
\hline Mean & 88.1 & 88.5 & 88.3 & 78.9 \\
\hline \#6 & 81.0 & 81.1 & 81.0 & 68.4 \\
\hline \#7 & Recovery satellite images & \\
\hline \#8 & 80.6 & Recall (\%) & $F_{1}$ score (\%) & IoU (\%) \\
\hline \#9 & 81.3 & 85.9 & 88.2 & 78.8 \\
\hline \#10 & 85.4 & 86.4 & 86.8 & 76.7 \\
\hline Mean & 81.3 & 87.4 & 84.2 & 72.8 \\
\hline Overall & 84.1 & 91.8 & 88.5 & 79.4 \\
accuracy & & 87.3 & 89.3 & 80.6 \\
\hline
\end{tabular}

The main challenges experienced in this case study were: (i) different textures of the building rooftops and in some cases their similarity with other land covers (e.g., bare soil) that made the change detection step challenging; (ii) inaccuracies in the OSM map data that influenced the change detection and extraction procedures (e.g., mismatches of the OSM building map with actual buildings in the satellite images, and missing boundaries for some buildings); (iii) the complexity of the scene to perform the building extraction task (e.g., mixture of slums and formal buildings even in the business district of the city, and buildings with various colors, shapes and other building characteristics). The proposed method produced $81.0 \%$ and $68.4 \%$ mean $F_{1}$-score and IoU accuracies, respectively, for the images belonging to the event time, and $87.4 \%$ and $77.7 \%$ mean $F_{1}$-score and IoU, respectively, for the recovery images. The lower accuracy for the event time images was due to presence of a large amount of debris in the areas just after the disaster. From the event time images, the test image \# 5 produced the best accuracy values with $88.3 \%$ $F_{1}$-score (Table 5.3), while the lowest accuracy belonged to image \#3 with a 
73.7\% $F_{1}$-score. The most important reason for the low performance was the similarity of the texture and color of the buildings with the damaged ones, which resulted in more false positives (i.e., red areas) in the results. In addition, image \#4 produced a $76.4 \% F_{1}$-score due to the presence of large amounts of debris around the intact buildings, which also led to more false positives. Also, the similarity of some of intact building rooftop colors and texture to debris resulted in more false negatives (i.e., blue colored areas). Moreover, images \#1 and \#2 demonstrated the efficiency of the proposed method in extracting buildings in dense urban areas, as well as a mixture of formal building and slum areas, by producing a $82.1 \%$ and $84.5 \% F_{1}$-score, respectively. However, one large dark-green colored building was not detected in this image using the proposed method, which could have two reasons: the lack of dark-green colored buildings in the training samples, and/or the building was detected only partially, which was later removed during post-processing, particularly during the implementation of the CRF method (Figure 5.7). Although CRF removed some pixels that were correctly classified as building in the previous step, it led to an overall increase in the $F_{1}$-score from $73.7 \%$ to $82.1 \%$ (Figure 5.7). Furthermore, image \#5 showed the robustness of the method in extracting even partially damaged building. In addition, the low $F_{1}$ score $(54.09 \%)$ and IoU accuracy $(37.1 \%)$ values produced by the network initially trained without fine-tuning for Image\# 1 shows the significance of this step in improving the performance of the final building database updating results (Figure 5.7). 

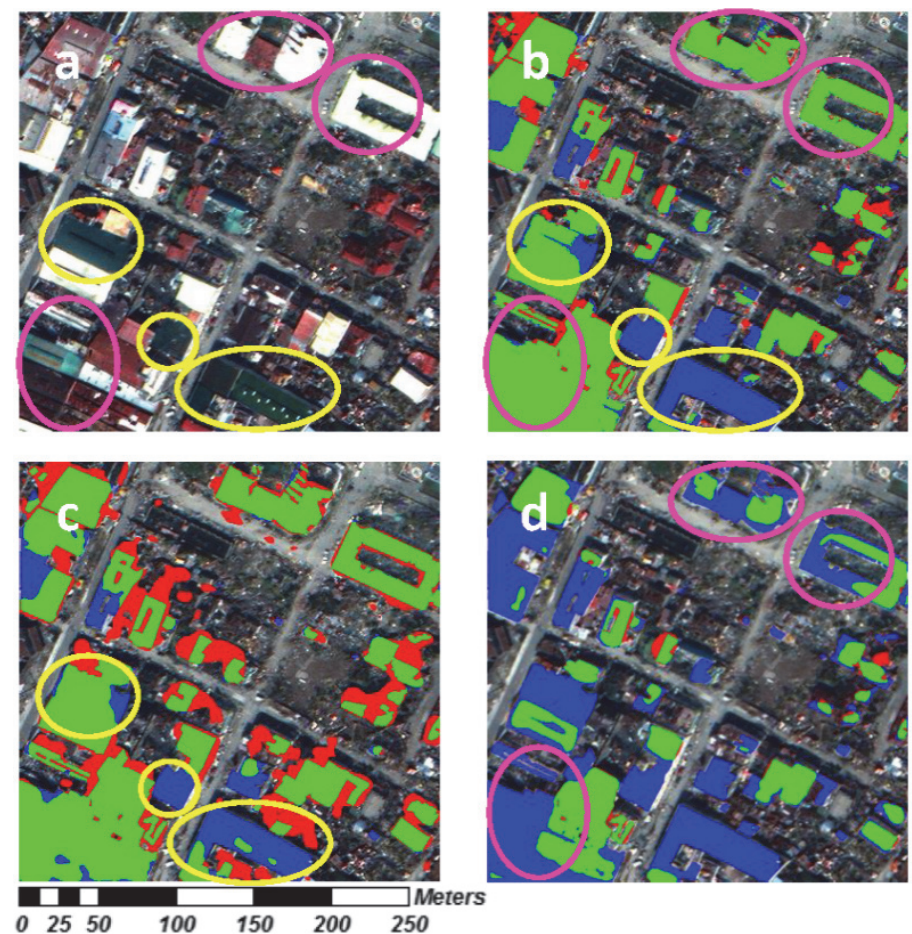

Figure 5.7 (a) Original test Image\#1, (b) ResUnet-CRF, (c) ResUnet, and (d) ResUnet without fine-tuning results. The areas within the yellow boundaries denote the dark green buildings to stress the effect of the CRF in the final result. The areas within the purple boundaries denote the inaccuracies in the results extracted using the network initially trained without fine-tuning. Green, red and blue pixels represent TP, FP and FN, respectively.

\subsection{Conclusion}

In this paper, we proposed a novel framework to update the post-disaster building database from VHR satellite imagery coupled with a leading online collaborative and open access map database, OSM. The approach was based on the automated training area generation for the proposed ResUnet-CRF network for building extraction. In addition, the proposed EDI and V-HOG indices confirmed a reliable performance in detecting changes of built-up areas from multi-temporal satellite imagery, to distinguish between damaged and intact buildings. This was then used as a preprocessing step in the automatic training area selection for the post-disaster building extraction (both for immediately after the disaster, and the recovery phase).

Experiments performed on ten test images selected from the study area (VHR images) demonstrated that the proposed approach produced robust results in updating the building database in different post-disaster scenarios, such as damaged, collapsed, reconstructed, newly built, and demolished buildings, 
using diverse building characteristics such as color, shape, and size of the buildings under challenging environmental conditions. Indeed, the efficacy of the proposed method was independent from building characteristics. Although the proposed method performed efficiently in this case study, it would likely produce even higher accuracies where OSM data are more accurate, such as in large cities. Assessing the impact of registering and modifying the OSM map and satellite images before training the network is also of interest for future studies. The inaccuracies in the OSM data (i.e. mismatching of the building footprint with the actual ones in the satellite images) led to smoothness in the edge of the buildings, which was mostly overcome through the CRF method. The CRF method showed a fairly good performance in refining the ResUnet results of the building boundaries; however, it did not perform well in the pixel brightness value-based refinements. This was also expected due to the complexity of the study area, in which the color variation was high and there was a strong similarity between the building and non-building classes in some parts of the images in terms of color and texture. In this case, in future research spatial context-based approaches can be used to overcome these drawbacks. The limitation of the proposed method was mainly associated with difficulties in detecting buildings that rarely occurred in the training set. For example, in a post-disaster scenario construction materials of the buildings may change to increase the resilience of the buildings, which may result in changes in the rooftop color and texture of the buildings which were not present in the training set used to build the network. However, this issue was limited by the proposed method by retraining the network using updated satellite images. Besides, the performance of the network can be improved by adding more image patches for training. In addition, the framework could be used to update building maps in a normal situation by implementing the proposed approach but excluding the change detection phase.

\subsection{References of Chapter 5}

Alam, F. I., Zhou, J., Liew, A. W., Jia, X., Chanussot, J., Gao, Y., 2018. Conditional random field and deep feature learning for hyperspectral image classification. IEEE Transactions on Geoscience and Remote Sensing, 1-17.

Armenakis, C., Leduc, F., Cyr, I., Savopol, F., Cavayas, F., 2003. A comparative analysis of scanned maps and imagery for mapping applications. ISPRS Journal of Photogrammetry and Remote Sensing, 57(5-6), 304-314.

Bai, Y., Mas, E., Koshimura, S., 2018. Towards operational satellite-based damage-mapping using u-net convolutional network: A case study of 2011 Tohoku earthquake-tsunami. Remote Sensing, 10(10), 1626.

Baltsavias, E. P., 2004. Object extraction and revision by image analysis using existing geodata and knowledge: current status and steps towards operational systems. ISPRS Journal of Photogrammetry and Remote Sensing, 58(3-4), 129-151. 
Bentabet, L., Jodouin, S., Ziou, D., Vaillancourt, J., 2003. Road vectors update using SAR imagery: a snake-based method. IEEE Transactions on Geoscience and Remote Sensing, 41(8), 1785-1803.

Bouziani, M., Goïta, K., He, D.-C., 2010. Automatic change detection of buildings in urban environment from very high spatial resolution images using existing geodatabase and prior knowledge. ISPRS Journal of Photogrammetry and Remote Sensing, 65(1), 143-153.

Brovelli, M., Zamboni, G., 2018. A new method for the assessment of spatial accuracy and completeness of OpenStreetMap building footprints. ISPRS International Journal of Geo-Information, 7(8), 289.

Canny, J., 1986. A Computational Approach to Edge Detection. IEEE Transactions on Pattern Analysis and Machine Intelligence, PAMI-8(6), 679-698.

Celik, T., 2009. Unsupervised change detection in satellite images using principal component analysis and k-means clustering. IEEE Geoscience and Remote Sensing Letters, 6(4), 772-776.

Chen, Z., Zhang, Y., Ouyang, C., Zhang, F., Ma, J., 2018. Automated landslides detection for mountain cities using multi-temporal remote sensing imagery. Sensors, 18(3), 821.

Cheng, G., Zhou, P., Yao, X., Yao, C., Zhang, Y., Han, J., 2016, 4-6 July 2016. Object detection in VHR optical remote sensing images via learning rotation-invariant HOG feature. Paper presented at the 2016 4th International Workshop on Earth Observation and Remote Sensing Applications (EORSA).

Choi, K., Lee, I., Kim, S., 2009. A feature based approach to automatic change detection from Lidar data in urban areas. Paper presented at the ISPRS Workshop on Laser scanning, Paris, France.

Dalal, N., Triggs, B., 2005. Histograms of oriented gradients for human detection. Paper presented at the Computer Society Conference on Computer Vision and Pattern Recognition (CVPR), San Diego, CA, USA.

Duarte, D., Nex, F., Kerle, N., Vosselman, G., 2018a. Multi-resolution feature fusion for image classification of building damages with convolutional neural networks. Remote Sensing, 10(10), 1636.

Duarte, D., Nex, F., Kerle, N., Vosselman, G., 2018b. Satellite image classification of building damages using airborne and satellite image samples in a deep learning approach. ISPRS Annals of Photogrammetry, Remote Sensing and Spatial Information Sciences, IV-2, 89-96.

Duarte, D., Nex, F., Kerle, N., Vosselman, G., 2019. Damage detection on building façades using multi-temporal aerial oblique imagery. ISPRS Ann. Photogramm. Remote Sens. Spatial Inf. Sci., IV-2/W5, 29-36.

Feng, T., Zhao, J., 2009. Review and Comparison: Building Extraction Methods Using High-Resolution Images. Paper presented at the 2009 Second International Symposium on Information Science and Engineering, 26-28 Dec. 2009.

Fiset, R., Cavayas, F., Mouchot, M.-C., Solaiman, B., Desjardins, R., 1998. Map-image matching using a multi-layer perceptron: The case of the road network. ISPRS Journal of Photogrammetry and Remote Sensing, 53(2), 76-84.

Geronimo, D., Lopez, A. M., Sappa, A. D., Graf, T., 2010. Survey of pedestrian detection for advanced driver assistance systems. IEEE Trans. Pattern. Anal. Mach. Intell., 32(7), 1239-1258. 
Ghaffarian, S., Ghaffarian, S., 2014a. Automatic building detection based on Purposive FastICA (PFICA) algorithm using monocular high resolution Google Earth images. ISPRS Journal of Photogrammetry and Remote Sensing, 97, 152-159.

Ghaffarian, S., Ghaffarian, S., 2014b. Automatic building detection based on supervised classification using high resolution Google Earth images. Paper presented at the The International Archives of Photogrammetry, Remote Sensing and Spatial Information Sciences, 40, 101.

Ghaffarian, S., Kerle, N., Filatova, T., 2018. Remote Sensing-Based Proxies for Urban Disaster Risk Management and Resilience: A Review. Remote Sensing, 10(11), 1760.

Ghaffarian, S., Turker, M., 2018. An improved cluster-based snake model for automatic agricultural field boundary extraction from high spatial resolution imagery. International Journal of Remote Sensing, 1-31.

Ghanea, M., Moallem, P., Momeni, M., 2016. Building extraction from highresolution satellite images in urban areas: recent methods and strategies against significant challenges. International Journal of Remote Sensing, 37(21), 5234-5248.

Gharibi, M., Arefi, H., Rastiveis, H., Hashemi, H., 2014. Automatic Building Map Updating Using Worldview-2 Stereo Images and Existing Outdated Building Map. ISPRS - International Archives of the Photogrammetry, Remote Sensing and Spatial Information Sciences, XL-2/W3, 131-136.

Gopalakrishnan, K., Khaitan, S. K., Choudhary, A., Agrawal, A., 2017. Deep Convolutional Neural Networks with transfer learning for computer visionbased data-driven pavement distress detection. Construction and Building Materials, 157, 322-330.

Holland, D. A., Sanchez-Hernandez, C., Gladstone, C., 2008. Detecting changes to topographic features using high resolution imagery. Paper presented at the XXIst ISPRS Congress, Beijing, China,.

Huang, Z., Pan, Z., Lei, B., 2017. Transfer Learning with Deep Convolutional Neural Network for SAR Target Classification with Limited Labeled Data. Remote Sensing, 9(9), 907.

Janalipour, M., Mohammadzadeh, A., 2016. Building damage detection using object-based image analysis and ANFIS from high-resolution image (Case Study: BAM Earthquake, Iran). Ieee Journal of Selected Topics in Applied Earth Observations and Remote Sensing, 9(5), 1937-1945.

Ji, M., Liu, L., Du, R., Buchroithner, M. F., 2019. A Comparative Study of Texture and Convolutional Neural Network Features for Detecting Collapsed Buildings After Earthquakes Using Pre- and Post-Event Satellite Imagery. Remote Sensing, 11(10), 1202.

Jiang, T., Xia, G., Lu, Q., 2017, 17-20 Sept. 2017. Sketch-based aerial image retrieval. Paper presented at the 2017 IEEE International Conference on Image Processing (ICIP). Beijing, China, 17-20 September 2017; pp. 3690-3694.

Jin, X., Davis, C. H., 2007. Vehicle detection from high-resolution satellite imagery using morphological shared-weight neural networks. Image and Vision Computing, 25(9), 1422-1431.

Jokar Arsanjani, J., Mooney, P., Zipf, A., Schauss, A., 2015. Quality Assessment of the Contributed Land Use Information from OpenStreetMap Versus Authoritative Datasets. 37-58. 
Jung, F., 2004. Detecting building changes from multitemporal aerial stereopairs. ISPRS Journal of Photogrammetry and Remote Sensing, 58(3-4), 187-201.

Ker, J., Wang, L., Rao, J., Lim, T., 2018. Deep Learning Applications in Medical Image Analysis. IEEE Access, 6, 9375-9389.

Knudsen, T., Olsen, B. P., 2003a. Automated change detection for updates of digital map databases. Photogrammetric Engineering \& Remote Sensing, 69(11), 1289-1296.

Knudsen, T., Olsen, B. P., 2003b. Automated change detection for updates of digital map databases. Photogramm. Eng. Remote Sensing, 69, 12891296.

Krähenbühl, P., Koltun, V., 2011. Efficient Inference in Fully Connected CRFS with Gaussian Edge Potentials. Paper presented at the Advances Neural Inf. Process Syst. (NIPS), Granada, Spain, 12-17 December 2011; pp. 109-117.

Le Bris, A., Chehata, N., 2011. Change detection in a topographic building database using submetric satellite images. Paper presented at the PIA11 - Photogrammetric Image Analysis, Munich, Germany, October 5-7.

Li, W., Wu, G., Du, Q., 2017a. Transferred Deep Learning for Anomaly Detection in Hyperspectral Imagery. IEEE Geoscience and Remote Sensing Letters, 14(5), 597-601.

Li, W., Wu, G., Zhang, F., Du, Q., 2017b. Hyperspectral Image Classification Using Deep Pixel-Pair Features. IEEE Transactions on Geoscience and Remote Sensing, 55(2), 844-853.

Li, X., Zhang, L., Du, B., Zhang, L., Shi, Q., 2017c. Iterative Reweighting Heterogeneous Transfer Learning Framework for Supervised Remote Sensing Image Classification. Ieee Journal of Selected Topics in Applied Earth Observations and Remote Sensing, 10(5), 2022-2035.

Liu, H., Yang, M., Chen, J., Hou, J., Deng, M., 2018. Line-Constrained Shape Feature for Building Change Detection in VHR Remote Sensing Imagery. ISPRS International Journal of Geo-Information, 7(10), 410.

Liu, Y., Piramanayagam, S., Monteiro, S. T., Saber, E., 2019. Semantic segmentation of multisensor remote sensing imagery with deep ConvNets and higher-order conditional random fields. Journal of Applied Remote Sensing, 13(01), 1.

Loncan, L., Almeida, L. B. D., Bioucas-Dias, J. M., Briottet, X., Chanussot, J., Dobigeon, N., Fabre, S., Liao, W., Licciardi, G. A., Simoes, M., Tourneret, J., Veganzones, M. A., Vivone, G., Wei, Q., Yokoya, N., 2015. Hyperspectral Pansharpening: A Review. IEEE Geoscience and Remote Sensing Magazine, 3(3), 27-46.

Lu, D., Mausel, P., Brondízio, E., Moran, E., 2004. Change detection techniques. International Journal of Remote Sensing, 25(12), 2365-2401.

Lyu, H., Lu, H., Mou, L., 2016. Learning a transferable change rule from a recurrent neural network for land cover change detection. Remote Sensing, 8(6), 506.

Malpica, J. A., Alonso, M. C., Papí, F., Arozarena, A., Martínez De Agirre, A., 2012. Change detection of buildings from satellite imagery and lidar data. International Journal of Remote Sensing, 34(5), 1652-1675.

Marmanis, D., Schindler, K., Wegner, J. D., Galliani, S., Datcu, M., Stilla, U., 2018. Classification with an edge: Improving semantic image 
segmentation with boundary detection. ISPRS Journal of Photogrammetry and Remote Sensing, 135, 158-172.

Matikainen, L., Hyyppä, J., Ahokas, E., Markelin, L., Kaartinen, H., 2010. Automatic detection of buildings and changes in buildings for updating of maps. Remote Sensing, 2(5), 1217-1248.

Mou, L., Ghamisi, P., Zhu, X. X., 2017a. Deep recurrent neural networks for hyperspectral image classification. IEEE Transactions on Geoscience and Remote Sensing, 55(7), 3639-3655.

Mou, L., Ghamisi, P., Zhu, X. X., 2017b, 23-28 July 2017. Fully conv-deconv network for unsupervised spectral-spatial feature extraction of hyperspectral imagery via residual learning. Paper presented at the 2017 IEEE International Geoscience and Remote Sensing Symposium (IGARSS). Fort Worth, TX, USA, 23-28 July 2017; pp. 5181-5184.

Nex, F., Duarte, D., Steenbeek, A., Kerle, N., 2019. Towards real-time building damage mapping with low-cost UAV solutions. Remote Sensing, 11(3), 287.

Pan, X., Zhao, J., 2018. High-Resolution Remote Sensing Image Classification Method Based on Convolutional Neural Network and Restricted Conditional Random Field. Remote Sensing, 10(6), 920.

Patel, M. I., Thakar, V. K., Shah, S. K., 2016. Image Registration of Satellite Images with Varying Illumination Level Using HOG Descriptor Based SURF. Procedia Computer Science, 93, 382-388.

Potts, R. B., 1952. Some generalized order-disorder transformations Mathematical proceedings of the cambridge philosophical society (Vol. 48, pp. 106-109): Cambridge Univ. Press.

Rottensteiner, F., 2007. Building change detection from digital surface models and multi-spectral images. Paper presented at the PIA07Photogrammetric Image Analysis, Munich, Germany, September 19-21.

Saha, S., Bovolo, F., Bruzzone, L., 2018. Destroyed-buildings detection from VHR SAR images using deep features. In Image and Signal Processing for Remote Sensing XXIV; International Society for Optics and Photonics: Berlin, Germany, 2018.

Shin, H., Roth, H. R., Gao, M., Lu, L., Xu, Z., Nogues, I., Yao, J., Mollura, D., Summers, R. M., 2016. Deep Convolutional Neural Networks for Computer-Aided Detection: CNN Architectures, Dataset Characteristics and Transfer Learning. IEEE Transactions on Medical Imaging, 35(5), 1285-1298.

Siebritz, L. A., Sithole, G., 1-3 July, 2014. Assessing the Quality of OpenStreetMap Data in South Africa in Reference to National Mapping Standards. Paper presented at the Second Africa GEO Conference, Cape Town, South Africa.

Singh, A., 1989. Review Article Digital change detection techniques using remotely-sensed data. International Journal of Remote Sensing, 10(6), 989-1003.

Sofina, N., Ehlers, M., 2016. Building Change Detection Using High Resolution Remotely Sensed Data and GIS. Ieee Journal of Selected Topics in Applied Earth Observations and Remote Sensing, 9(8), 3430-3438.

Tian, J., Chaabouni-Chouayakh, H., P., R., 2011. 3D building change detection from high resolution spaceborne stereo imagery. Paper presented at the International Workshop M2RSM, Xiamen, China. 
Torrione, P. A., Morton, K. D., Sakaguchi, R., Collins, L. M., 2014. Histograms of Oriented Gradients for Landmine Detection in Ground-Penetrating Radar Data. IEEE Transactions on Geoscience and Remote Sensing, 52(3), 1539-1550.

Unisdr. 2015, 14-18 March. Sendai framework for disaster risk reduction 2015 - 2030. Paper presented at the Third World Conf. Disaster Risk Reduction, Sendai, Japan.

Vetrivel, A., Gerke, M., Kerle, N., Nex, F., Vosselman, G., 2017. Disaster damage detection through synergistic use of deep learning and 3D point cloud features derived from very high resolution oblique aerial images, and multiple-kernel-learning. ISPRS Journal of Photogrammetry and Remote Sensing.

Vetrivel, A., Gerke, M., Kerle, N., Vosselman, G., 2016. Identification of structurally damaged areas in airborne oblique images using a visual-bagof-words approach. Remote Sensing, 8(3), 231.

Walter, V., 1999. Comparison of the potential of different sensors for an automatic approach for change detection in GIS databases Digital Images and GIS. International Workshop ISD (pp. 47-63). Lecture Notes in Computer Science: Springer.

Wang, Y., Liang, B., Ding, M., Li, J., 2018. Dense Semantic Labeling with Atrous Spatial Pyramid Pooling and Decoder for High-Resolution Remote Sensing Imagery. Remote Sensing, 11(1), 20.

Xiao, L., Zhang, Y., Peng, G., 2018. Landslide Susceptibility Assessment Using Integrated Deep Learning Algorithm along the China-Nepal Highway. Sensors, 18(12), 4436.

Xu, S., Vosselman, G., Oude Elberink, S., 2015. Detection and Classification of Changes in Buildings from Airborne Laser Scanning Data. Remote Sensing, 7(12), 17051-17076.

Xu, Y., Yu, G., Wang, Y., Wu, X., Ma, Y., 2016. A Hybrid Vehicle Detection Method Based on Viola-Jones and HOG + SVM from UAV Images. Sensors, 16(8), 1325.

Yang, W., Yin, X., Xia, G., 2015. Learning High-level Features for Satellite Image Classification With Limited Labeled Samples. IEEE Transactions on Geoscience and Remote Sensing, 53(8), 4472-4482.

Yuan, M., Liu, Z., Wang, F., 2019. Using the wide-range attention U-Net for road segmentation. Remote Sensing Letters, 10(5), 506-515.

Zhang, Z., Liu, Q., Wang, Y., 2018a. Road Extraction by Deep Residual U-Net. IEEE Geoscience and Remote Sensing Letters, 15, 749-753.

Zhang, Z., Vosselman, G., Gerke, M., Tuia, D., Yang, M. Y., 2018b. Change Detection between Multimodal Remote Sensing Data Using Siamese CNN. arXiv 2018, arXiv: 1807.09562.

Zhao, B., Zhong, Y., Xia, G., Zhang, L., 2016. Dirichlet-Derived Multiple Topic Scene Classification Model for High Spatial Resolution Remote Sensing Imagery. IEEE Transactions on Geoscience and Remote Sensing, 54(4), 2108-2123.

Zhao, Y., Ma, J., Li, X., Zhang, J., 2018. Saliency Detection and Deep LearningBased Wildfire Identification in UAV Imagery. Sensors (Basel), 18(3).

Zhu, X. X., Tuia, D., Mou, L., Xia, G., Zhang, L., Xu, F., Fraundorfer, F., 2017. Deep Learning in Remote Sensing: A Comprehensive Review and List of Resources. IEEE Geoscience and Remote Sensing Magazine, 5(4), 8-36. 
Zielstra, D., Zipf, A., 11-14 May 2010. A comparative study of proprietary geodata and volunteered geographic information for Germany. Paper presented at the 13th AGILE International Conference on Geographic Information Science, Guimaraes, Portugal. 


\section{Chapter 6 - Post-disaster recovery monitoring with Google Earth Engine ${ }^{5}$}

\footnotetext{
5 This chapter is based on:

- Ghaffarian, S.; Rezaie Farhadabad, A.; Kerle, N., 2020. Post-disaster recovery monitoring with Google Earth Engine. Appl. Sci., 10, 4574.
} 


\section{Abstract}

Post-disaster recovery is a complex process in terms of measuring its progress after a disaster and understanding its components and influencing factors. During this process, disaster planners and governments need reliable information to make decisions towards building the affected region back to normal (pre-disaster), or even improved, conditions. Hence, it is essential to use methods to understand the dynamics/variables of the post-disaster recovery process, and rapid and cost-effective data and tools to monitor the process. Google Earth Engine (GEE) provides free access to vast amounts of remote sensing (RS) data and a powerful computing environment in a cloud platform, making it an attractive tool to analyze earth surface data. In this study we assessed the suitability of GEE to analyze and track recovery. To do so, we employed GEE to assess the recovery process over a three-year period after Typhoon Haiyan, which struck Leyte island, in the Philippines, in 2013. We developed an approach to (i) generate cloud and shadow-free image composites from Landsat 7 and 8 satellite imagery and produce land cover classification data using the Random Forest method, and (ii) generate damage and recovery maps based on post-classification change analysis. The method produced land cover maps with accuracies $>88 \%$. We used the model to produce damage and three time-step recovery maps for 62 municipalities on Leyte island. The results showed that most of the municipalities had recovered after three years in terms of returning to the pre-disaster situation based on the selected land cover change analysis. However, more analysis (e.g., functional assessment) based on detailed data (e.g., land use maps) is needed to evaluate the more complex and subtle socio-economic aspects of the recovery. The study showed that GEE has good potential for monitoring the recovery process for extensive regions. However, the most important limitation is the lack of very-high-resolution RS data that are critical to assess the process in detail, in particular in complex urban environments. 


\subsection{Introduction}

Natural disasters have devastating impacts on infrastructure, business sectors, and people in the affected region. Between 1998 and 2017, more than 5.7 billion people were affected by disasters, and more than one million people were killed by such events, while a total loss of USD 2.9 trillion was reported (CRED 2018). Recovery starts after the immediate post-disaster response phase, mainly search and rescue operations, have concluded. Post-disaster recovery is the process of reconstructing communities in all their aspects (e.g., physical, economic, social, and environmental) in order to return life, livelihoods, and the built environment to their pre-impact (Burton et al. 2011), or even better, states, as per the Sendai Framework (UNISDR 2015). Recovery can take years or even decades, and is the least studied phase of the disaster management cycle. Moreover, it has been considered as a proxy to evaluate resilience (Ghaffarian et al. 2018; Sheykhmousa et al. 2019), with resilient communities recovering faster after a disaster. However, a recent study argued that this assumption may not be true in all cases, and there is a need for more studies to explore the relationship (Kerle et al. 2019a). Accordingly, providing information about the status of the damage and the reconstruction and recovery process after a disaster is vital to support decision-makers and planners, but also to support post-event risk updating efforts.

Remote sensing (RS) data and techniques have been extensively used for different aspects of disaster risk management (DRM) (Ghaffarian et al. 2018), from quantification of social (Ebert et al. 2009) and physical (Harb et al. 2015) vulnerabilities, to rapid damage assessments (Kerle et al. 2019b) using satellite (Vetrivel et al. 2016b), airborne (Vetrivel et al. 2016a), and Unmanned Aerial Vehicle (UAV) images (Nex et al. 2019a), and numerous data analysis methods have been developed. Conversely, only recently have a small number of studies focused on the recovery phase (Ghaffarian and Kerle 2019b; Sheykhmousa et al. 2019). Recently, RS data also started to be used for disaster recovery monitoring; however, most of these studies used RS for physical recovery assessment (Ghaffarian et al. 2018). For instance, Brown et al. (Brown et al. 2010) developed an indicator-based methodology to monitor and evaluate post-disaster recovery based on high-resolution RS imagery, particularly IKONOS and QuickBird satellite images, in addition to field surveys and internet-based statistic data sets. They implemented image processing techniques for change detection (i.e., land cover changes) and building-based recovery/reconstruction analysis. They evaluated the potential of RS data for recovery assessment. In a different study, Burton et al. (Burton et al. 2011) used the repeat photography method to evaluate post-Katrina recovery in Mississippi. They took photographs every six months over a three-year period. Then, by assigning scores to each scene in terms of change and recovery, they generated a map for recovery assessment for the entire region. Night-time 
light satellite images have been also used for damage and recovery analysis, and it has been demonstrated that there is a close relationship between light intensity and economic activity (Sutton et al. 2007). However, the night-time light satellite images are only useful for urban area recovery assessments.

Brown et al. (Brown et al. 2011) developed a model to assess the damage and early recovery using RS data and ground survey tools after the 2008 Wenchuan earthquake in China. The recovery step of their study included buildings, accessibility, power, and water livelihoods assessments. In their study, only the Normalized Differential Vegetation Index (NDVI) computation was implemented automatically, and other information, such as building change detection and accessibility assessments, were generated manually. Costa Vieira and Kerle (Costa Viera and Kerle 2014) studied urban recovery using geospatial data for the firework disaster in Enschede, the Netherlands, 2000. They mainly employed very high-resolution aerial images to extract information about the building morphology, such as building density, shape, and size, and concentration of road networks as proxies. In addition they proposed a proxy to measure the quality of housing based on the energy loss indicator of the buildings. Hoshi et al. (Hoshi et al. 2014) used ground survey information in combination with RS, employing visual interpretation and binary classification methods to monitor post-disaster urban recovery. Contreras et al. (Contreras et al. 2016) integrated RS (high-resolution satellite/aerial imagery) and ground observations to assess the recovery process in the Italian city of L'Aquila after the 2009 earthquake. They showed that using RS data for recovery monitoring reduces the required ground fieldwork.

Recent studies have focused on the extraction of proxies for post-disaster recovery assessment by land cover and land use classification and change detection of very high-resolution images using advanced machine learning methods (Kerle 2016). Sheykhmousa et al. (Sheykhmousa et al. 2019) implemented a Support Vector Machine (SVM)-based method to produce land cover and land use classification maps for before, event/just after, and years after the disaster. They assessed the recovery processes by monitoring the changes in land cover and use maps. They also introduced the concept of positive and negative recoveries based on the observed change patterns. Furthermore, Kerle et al. (Kerle et al. 2019a) developed a conceptual framework for RS-based recovery monitoring and then integrated it with statistical economic analysis to assess the resilience-centered development interventions. They hypothesized that the speed of recovery is a proxy for resilience assessment; however, for some areas their results showed a negative relationship between image-derived speed of the recovery and resilience calculated based on household surveys. They employed the Extreme Gradient Boosting (XGBoost) method (Chen and Guestrin 2016; Georganos et al. 2018) to classify the very high-resolution images to extract RS-based 
proxies. They concluded that although RS can provide useful information in addition to socio-economic survey data, the high cost of very high-resolution images and the need for high computational processing power are the limitations of utilizing RS data. Moreover, deep learning, in particular convolutional neural networks (CNN), has become the state-of-the-art method in computer vision and RS data analysis, and has also been used for postdisaster damage and recovery assessments (Duarte et al. 2018; Kerle et al. 2019b; Nex et al. 2019b). For instance, a new image-patch generation approach was developed to train a CNN-based network simultaneously with multi-temporal satellite images and assess recovery (Ghaffarian and Kerle 2019a). In addition, free OpenStreetMap building footprints were employed to automatically generate training data from very high-resolution satellite images for a pixel-level deep learning method for post-disaster building database updating (Ghaffarian et al. 2019). The study showed that the proposed approach significantly decreased the manual work of training area collection, while maintaining the accuracy of the detected damaged, reconstructed, and newly constructed buildings at a high level. However, extensive computational power is needed to execute deep learning methods.

Most of the developed RS-based approaches in post-disaster damage and recovery assessments focus on the use of costly very high-resolution data that require extensive digital storage and computing capacity to make use of them. In recent years, freely available RS images made available through platforms such as Google Earth have attracted the attention of researchers to extract useful information (Ghaffarian and Ghaffarian 2014a, 2014b). In addition, cloud-based platforms such as Google Earth Engine (GEE) provide free RS data and computing power with a coding environment to develop and implement user-defined methods and process the data (Gorelick et al. 2017; Kumar and Mutanga 2018). Freely available, low- to medium-resolution images by data providers (e.g., Copernicus) are mainly collected in these platforms. The potential of such systems has been studied for vegetation cover change analysis (Xie et al. 2019), land cover and land use classification (Ge et al. 2019; Stromann et al. 2019), change detection/analysis (Canty et al. 2019; Sidhu et al. 2018), wetland map generation (Mahdianpari et al. 2018; Wu et al. 2019), rangeland and crop monitoring (Xiong et al. 2017; Zhou et al. 2020), and in the DRM domain for flood prevention and emergency response (Liu et al. 2018), drought assessment (Sazib et al. 2018), and wildfire progress mapping (Crowley et al. 2019). However, they have not yet been studied to monitor the post-disaster recovery process. Furthermore, there is a need for higher spatial resolution images for detailed analysis (e.g., functional assessments), especially in urban areas.

The aim of this study was to test the suitability of GEE for a large-scale postdisaster recovery assessment. The big time series Landsat 7 and 8 data 
available on GEE were used to first generate image composites for the before, event/just after, and post-disaster times (i.e., three time-steps after the disaster) to obtain cloud-free images, by adapting the method developed by De Alban et al. (De Alban et al. 2018). Then, the Random Forest classifier available on the cloud computing platform was employed to classify the generated images and monitor the land cover changes during the recovery process. The entire Leyte island (with an area of ca. $7300 \mathrm{~km}^{2}$ ) in the Philippines was selected to test the developed approach, and municipalitybased damage and recovery maps for the entire island were generated for the selected time epochs. Leyte region was hit by Super Typhoon Haiyan on 8 November 2013. As one of the strongest typhoons on record worldwide, it resulted in more than 6000 fatalities, and total damages were estimated at ca. USD 2.2 billion (NDRRMC 2014).

\subsection{Materials and Methods}

\subsubsection{Case Study and Google Earth Engine Data}

Leyte province, located in the Eastern Visayas, is the seventh largest island in the Philippines (Figure 6.1). With a population of approximately 250,000, Tacloban is the biggest city and the capital of Leyte, which in total houses ca. 1.7 million people. The region has a tropical climate with two slightly different rainfall patterns during a year. While most of Leyte island has an even annual rainfall distribution, some eastern parts have a pronounced maximum rainfall from November to January. Typhoon Haiyan (also known as Typhoon Yolanda in the region) passed over Leyte island close to Tacloban City on 8 November 2013. Tacloban was hit by the full force of the typhoon, which caused massive destruction in the city. A state of emergency was declared in Tacloban and the typhoon caused a storm surge of up to 5 m (NDRRMC 2014).

Since Leyte island is a tropical region and is covered by clouds most of the time, we first aimed at using Synthetic Aperture Radar (SAR) data. However, only Landsat images were available and found suitable for our study in the GEE data sets for the time of the disaster and recovery processes. Accordingly, atmospherically corrected surface reflectance Landsat 7 and 8 satellite images with $30 \mathrm{~m}$ spatial resolution were used to map the selected land cover types and implement the developed approach for post-disaster recovery assessment (Table 6.1). In total, 446 Landsat images were employed in this study. In particular the potential of big geospatial time-series data (i.e., Landsat 7 and 8 images) available on the cloud was used to generate the best-available-pixel image composites (explained in the methodology section) for the pre-disaster, event time, and post-disaster time steps over Leyte island to minimize/remove the cloud and shadow areas. 


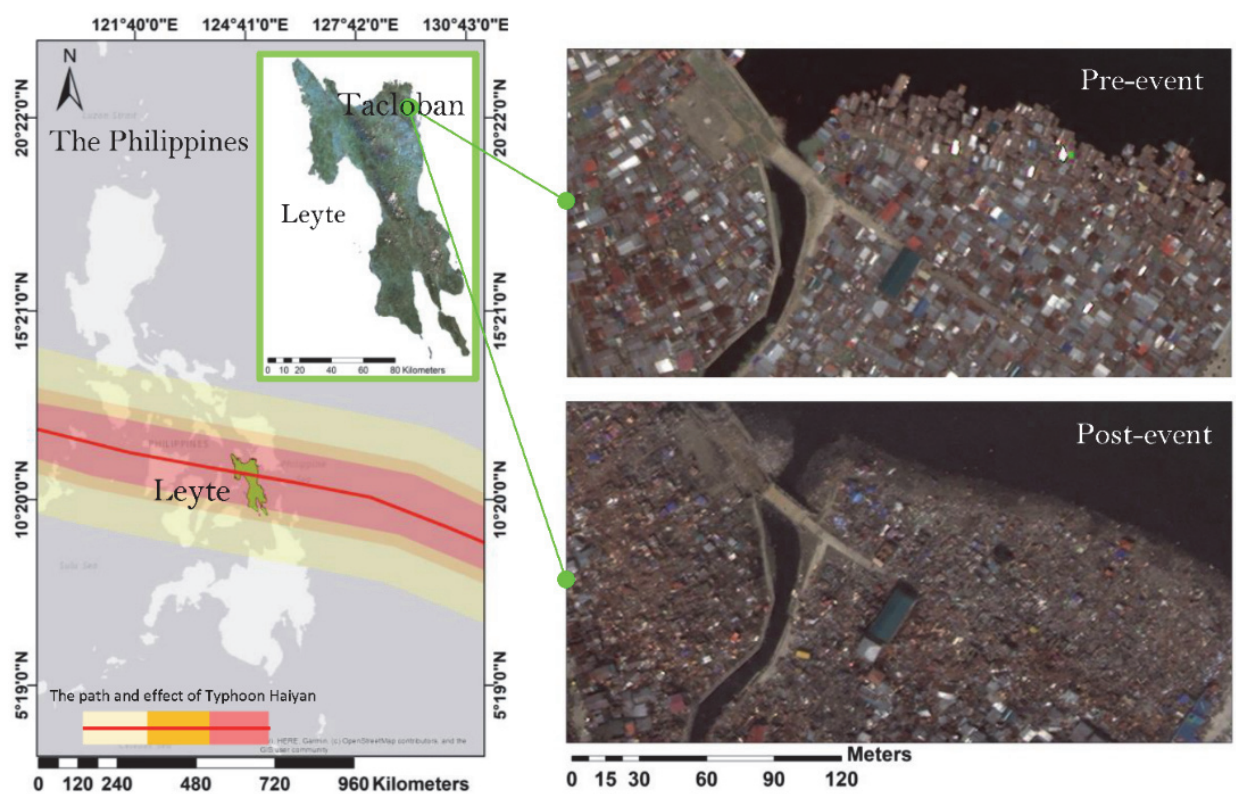

Figure 6.1 An overview of the Philippines showing the path of Typhoon Haiyan and the location of Leyte island. Pre-event image and an image acquired three days after the disaster for Tacloban.

Table 6.1 Satellite images used in this study.

\begin{tabular}{|c|c|c|c|c|c|}
\hline ID & Timeline & $\begin{array}{l}\text { Acquisition } \\
\text { Dates }\end{array}$ & $\begin{array}{c}\text { Number } \\
\text { of } \\
\text { Images } \\
\text { Collected }\end{array}$ & $\begin{array}{c}\text { Satellite } \\
\text { Platforms }\end{array}$ & $\begin{array}{c}\text { Spatial } \\
\text { Resolution }\end{array}$ \\
\hline TO & Before Haiyan & $\begin{array}{c}2013-05-01 \text { to } \\
2013-10-28\end{array}$ & 68 & \multirow{5}{*}{$\begin{array}{c}\text { Landsat } 7 \\
\text { ETM+ and } \\
\text { Landsat } 8 \\
\text { OLI }\end{array}$} & \multirow{5}{*}{$30 \mathrm{~m}$} \\
\hline T1 & Event time & $\begin{array}{c}2013-11-10 \text { to } \\
2014-03-31\end{array}$ & 56 & & \\
\hline T2 & Post-Haiyan 1 & $\begin{array}{c}2014-06-01 \text { to } \\
2014-12-30\end{array}$ & 104 & & \\
\hline T3 & Post-Haiyan 2 & $\begin{array}{c}2015-06-01 \text { to } \\
2015-12-30\end{array}$ & 109 & & \\
\hline T4 & Post-Haiyan 3 & $\begin{array}{c}2016-06-01 \text { to } \\
2016-12-30\end{array}$ & 109 & & \\
\hline
\end{tabular}

\subsubsection{Reference Data and Land Cover Classes}

We defined the land cover classes based on the local knowledge of the authors about the study area, and by considering the capacity/potential of the Landsat images (i.e., spatial and spectral resolutions) to identify different classes through field verification and visual interpretation of high-resolution satellite imagery acquired from various platforms (i.e., WorldView1-3, GeoEye 1, 
Pleiades) and images available from Google Earth Pro. We identified five land cover classes: forest/trees, built-up, crop land, water body, and other (i.e., non-tree vegetation, bare land, and debris/rubble) (Table 6.2), and the corresponding regions of interests (ROIs) were collected for training and testing of the classification method using the tools available within GEE.

Table 6.2 Description of the selected land cover classes.

\begin{tabular}{|c|c|c|}
\hline ID & Land Cover & Description \\
\hline 1 & Forest/Trees & $\begin{array}{c}\text { All types of trees (e.g., forest, palm and banana), tree } \\
\text { canopy coverage }>30 \%\end{array}$ \\
\hline 2 & Built-up & $\begin{array}{c}\text { Any type of developed lands such as buildings, roads, } \\
\text { impervious surfaces }\end{array}$ \\
\hline 3 & Crop land & $\begin{array}{c}\text { Agricultural fields with any non-tree crop type } \\
\text { plantation }\end{array}$ \\
\hline 4 & Water body & $\begin{array}{c}\text { Bodies of water including lakes, oceans, rivers and } \\
\text { flooded areas }\end{array}$ \\
\hline 5 & Other & $\begin{array}{c}\text { All other land cover classes (i.e., non-tree vegetation, } \\
\text { bare land, rubble and debris) }\end{array}$ \\
\hline
\end{tabular}

\subsubsection{Methods}

The developed approach for post-disaster recovery assessment has three main steps (Figure 6.2): (i) land cover classification of the pre-disaster, event time, and post-disaster images, (ii) change detection of pre-disaster and event time classified images to obtain the damage map, and (iii) change analysis of the post-disaster classified images and the damage map to obtain the recovery maps at T2-T4. Generating the land cover maps from the Landsat 7 and 8 satellite images with GEE consisted of three main stages: image composite generation, image classification, and accuracy assessment. 


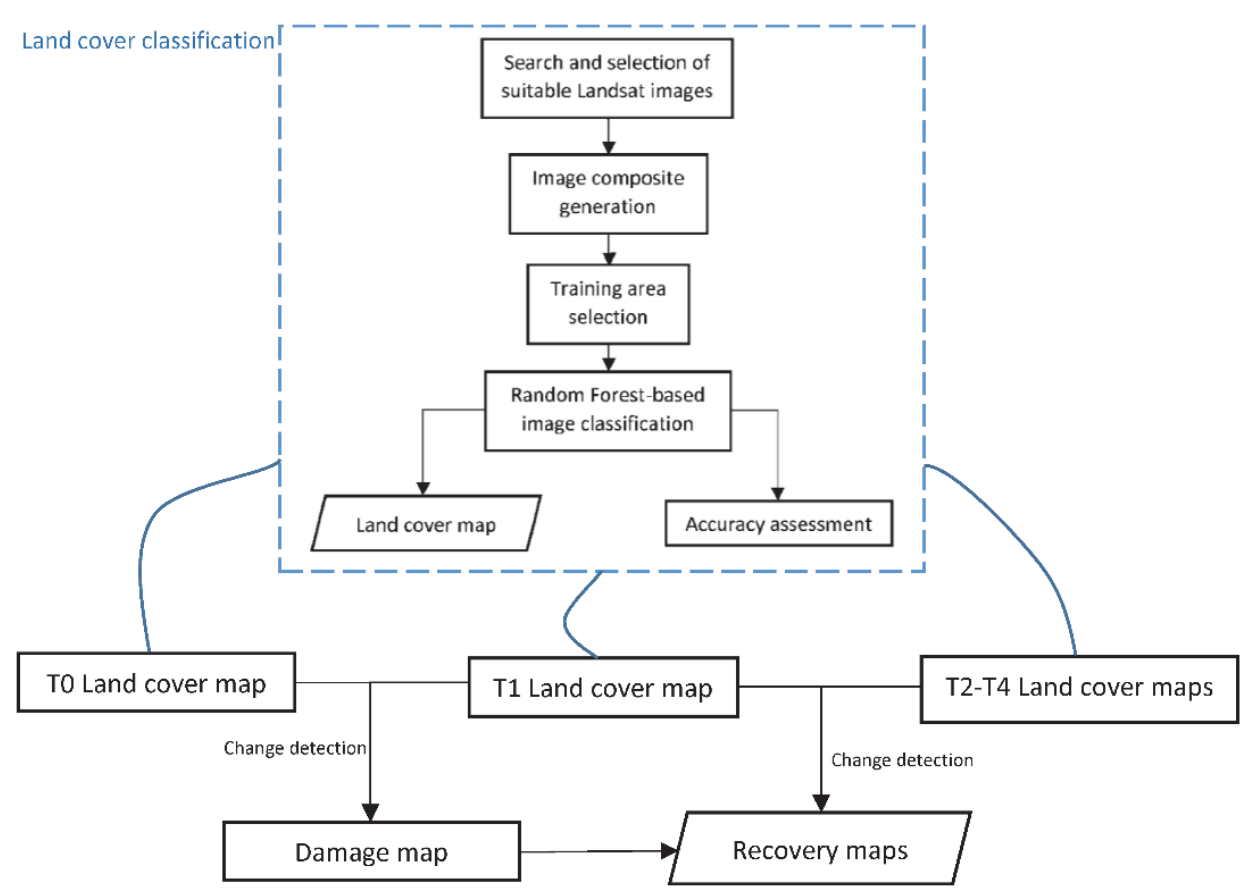

Figure 6.2 The proposed framework for post-disaster recovery monitoring.

\subsubsection{Image Composite Generation}

We generated the best-available-pixel image composites for the T0-T4 time steps by extracting the best observations from several Landsat 7 and 8 images for our case study (Leyte island), using pixel-based image compositing within the GEE platform, and implemented using rule-based criteria such as dates of acquisition and exclusion of clouds and shadows (Griffiths et al. 2013; Hansen and Loveland 2012; White et al. 2014; Wulder et al. 2012). To do so, we adapted a script within the GEE environment provided by De Alban et al. (De Alban et al. 2018). The generated Landsat image composites were exported for subsequent use in the image classification. The developed script needs user-defined inputs and parameters: the coordinates and extent of Leyte island, the number of years, starting and ending date range for the selected region (i.e., Leyte), the thresholds for cloud detection and masking (10\% or less) and shadows ( $z$-score $=-1$ ), and the image collection used, (i.e., Landsat 7 ETM+ and Landsat $8 \mathrm{OLI})$. Then the areas that were masked out in the previous step were filled by the median of the selected image pixels (De Alban et al. 2018; Reductions. 2019). The scripts developed for this study are provided as supplementary materials in this paper.

In addition, we calculated five well-known indices from the Landsat composite images and added to the available Landsat bands to be used in the classification step as follows: 
Normalized Difference Vegetation Index (NDVI; (Tucker 1979)) using Equation (1):

$$
N D V I=\frac{N I R-R e d}{N I R+R e d}
$$

The Soil-Adjusted Total Vegetation Index (SATVI; (Marsett et al. 2006)) using Equation (2):

$$
S A T V I=\frac{S W I R 1-\text { Red }}{S W I R 1+\text { Red }+0.1} *(1.1-S W I R 2 / 2.0)
$$

Enhanced Vegetation Index (EVI; (Huete et al. 2002; Huete et al. 1997)) using Equation (3):

$$
E V I=2.5 * \frac{N I R-\text { Red }}{N I R+(6.0 * \text { Red })-(7.5 * \text { Blue })+1.0}
$$

Land Surface Water Index (LSWI; (Gao 1996; Jurgens 1997)) using Equation (4):

$$
L S W I=\frac{N I R-S W I R 1}{N I R+S W I R 1}
$$

Normalized Difference Tillage Index (NDTI; (Van Deventer et al. 1997)) using Equation (5):

$$
N D T I=\frac{S W I R 1-S W I R 2}{S W I R 1+S W I R 2}
$$

where Blue is the blue band, Red is the red band, NIR is the near-infrared band, SWIR1 is the shortwave infrared 1 band, and SWIR2 is the shortwave infrared 2 band of the Landsat image.

NDVI was shown to be a useful tool for distinguishing green vegetation areas from other land cover types; LSWI is for detecting water bodies and when combined with NDVI improves the performance of separating crop lands and forests (Xiao et al. 2002). SATVI, EVI, and NDTI have been shown to be effective tools for identifying forest and crop types from other land cover classes (De Alban et al. 2018; Torbick et al. 2016).

\subsubsection{Image Classification}

The final stacked/combined images for each time step consisted of seven Landsat bands (i.e., Blue, Green, Red, NIR, SWIR1, SWIR2, and TIR) and the computed indices (i.e., NDVI, EVI, SATVI, NDTI, and LSWI). The combined images were stored under the "Asset" tab of the GEE code editor platform to be later used for training and testing ROI selection/extraction.

We collected regions of interest (ROIs)/training areas from the images, using the tools within GEE in a way that each class had at least 40 polygons. These selections were based on field verifications and visual interpretations from high-resolution Google Earth Pro historical images and high-resolution satellite images acquired by different sensors (i.e., WorldView1-3, GeoEye1, Pleiades), and Landsat true- and false-color images to obtain final robust training and 
testing datasets. From the collected ROIs, 70\% were randomly selected for training the classifier method and the rest were used for testing purposes. We used Random Forest, as an ensemble machine learning algorithm, to classify the images based on the collected training samples. Random Forest has been widely used in RS image processing for different applications (Belgiu and Drăgut 2016), including land cover and land use classification (Abdi 2019; Gislason et al. 2006), landslide detection (Stumpf and Kerle 2011), and hyperspectral image classification (Ham et al. 2005), due to providing efficient and accurate results. It is also one of the machine learning-based methods available and ready to use in GEE. This approach has also been used by other researchers and its ability to produce accurate classification results has been demonstrated (Kelley et al. 2018; Oliphant et al. 2019; Shelestov et al. 2017; Teluguntla et al. 2018). In addition, Random Forest was selected as the best classifier among several advanced algorithms, including SVM and neural networks, for classifying hundreds of different real-world datasets (FernándezDelgado et al. 2014).

Random Forest grows multiple decision trees on randomly selected training subsets. In addition, it takes the advantage of the bootstrapping approach to construct the decision trees from training samples and input variables at every node. The constructed numerous decision tress then go through the majority voting mechanism to obtain the final classification result. This strategy contributes to overcoming the limitations of single tree-based classifiers such as overfitting, and makes it less sensitive to noise. In the current study we executed Random Forest with 100 decision trees per class, a 0.5 fraction number to bag per tree, and 10 for the minimum size of a terminal node.

\subsubsection{Accuracy Assessment}

The overall user's and producer's accuracies were calculated for each time step classified maps (i.e., T0-T4) to assess the accuracy of the produced land cover classification results. A proportion of $30 \%$ of the collected ROIs for each class were selected using stratified random sampling for testing and computing the accuracy measures (Marsett et al. 2006). To do so, we adapted the code script used by De Alban et al. (De Alban et al. 2018) and executed it within the GEE environment. Selecting the training and ground truth ROIs was challenging for some classes due to similarities between the land cover classes (e.g., crop land and vegetation classes). We tried to minimize the effect of these inaccuracies using different sources (e.g., very high-resolution satellite images) in selecting the ROIs. In addition, considering debris/rubble as the damaged area may cause inaccuracies, e.g., in the case of storm surge that may relocate/wash debris to other regions covering the intact built-up area (Ghaffarian and Kerle 2019). 


\subsubsection{Change Analysis}

Final damage and recovery maps were generated based on the amount of change in the forest, built-up, and crop land classes. The changes were computed at the municipality level, and the damage map was generated by comparing the T0 and T1 land cover classification results; also, the recovery maps for each post-disaster time step (T2-T4) were computed by comparing the T1 and T2-T4 land cover classification results.

\subsection{Results and Discussion}

The proposed approach produced overall accuracies of $92.8 \%, 88.6 \%, 93.8 \%$, $94.6 \%$, and $89.0 \%$ for land cover classifications of the T0, T1, T2, T3, and T4 images, respectively (Table 6.3). In addition, Figure 6.3 shows the original true-color image composites and the results of the land cover classification for the T0-T4 time steps. Visual interpretation of the results and the high accuracy rates demonstrate the robustness of the approach and the Random Forest classifier for land cover classification for the defined classes in such a case study. However, the producer's accuracy values of the built-up and the other (including non-tree vegetation, bare land, and debris) classes for T1 and T4 images were $76.5 \%$ and $75.4 \%$, respectively, which shows the relatively high omission errors for those classes. Relocation of palm tree fronds by the typhoon made distinguishing trees, crop land, and other (including debris) classes more difficult for the event time, and resulted in the lowest overall accuracy of $88.6 \%$ for the T1 image classification. Furthermore, the generated composite images may still include cloud coverage. For example, the presence of clouds in the T4 image even after image composition generation resulted in some inaccuracies that produced the second-lowest overall accuracy of $89.0 \%$ among other images. The developed method produced accurate results in classifying builtup, water body, and forest/trees classes in most of the images. 
Table 6.3 The land cover classification accuracies for $\mathrm{T} 0, \mathrm{~T} 1, \mathrm{~T} 2, \mathrm{~T} 3$, and $\mathrm{T} 4$ time epochs for Leyte island. PA-producer's accuracy; UA-user's accuracy; OA-overall accuracy; Non-tv-Non-tree vegetation.

\begin{tabular}{|c|c|c|c|c|c|c|}
\hline \multicolumn{2}{|c|}{ Time/Class } & \multirow{2}{*}{$\begin{array}{c}\text { Trees } \\
90.7 \\
\end{array}$} & \multirow{2}{*}{$\begin{array}{c}\text { Built- } \\
\text { Up } \\
100\end{array}$} & \multirow{2}{*}{$\begin{array}{r}\text { Crop } \\
\text { Land } \\
93.0\end{array}$} & \multirow{2}{*}{$\begin{array}{c}\text { Water } \\
\text { Body } \\
100\end{array}$} & \multirow{2}{*}{$\begin{array}{c}\text { Other (Non-tv, } \\
\text { Bare Land, } \\
\text { Debris) } \\
86.7\end{array}$} \\
\hline \multirow{3}{*}{$\begin{array}{l}\text { Pre-disaster } \\
\text { (TO) }\end{array}$} & UA(\%) & & & & & \\
\hline & $\mathrm{PA}(\%)$ & 87.3 & 100 & 84.6 & 100 & 85.8 \\
\hline & OA(\%) & \multicolumn{5}{|c|}{92.8} \\
\hline \multirow{3}{*}{$\begin{array}{l}\text { Event time } \\
\text { (T1) }\end{array}$} & UA(\%) & 98.4 & 94.8 & 85.1 & 100 & 71.9 \\
\hline & PA(\%) & 84.1 & 100 & 84.9 & 100 & 76.5 \\
\hline & OA(\%) & \multicolumn{5}{|c|}{88.6} \\
\hline \multirow{3}{*}{$\begin{array}{c}\text { Post-disaster } \\
\text { (T2) }\end{array}$} & UA(\%) & 96.4 & 89.1 & 90.6 & 99.9 & 90.3 \\
\hline & $\mathrm{PA}(\%)$ & 88.4 & 95.7 & 90.6 & 99.8 & 96.4 \\
\hline & OA(\%) & \multicolumn{5}{|c|}{93.8} \\
\hline \multirow{3}{*}{$\begin{array}{c}\text { Post-disaster } \\
\text { (T3) }\end{array}$} & UA(\%) & 95.7 & 98.5 & 84.8 & 100 & 86.5 \\
\hline & $\mathrm{PA}(\%)$ & 93.1 & 97.8 & 98.7 & 100 & 91.3 \\
\hline & OA(\%) & \multicolumn{5}{|c|}{94.6} \\
\hline \multirow{3}{*}{$\begin{array}{c}\text { Post-disaster } \\
\text { (T4) }\end{array}$} & $\mathrm{UA}(\%)$ & 90.1 & 81.0 & 86.3 & 99.5 & 86.7 \\
\hline & $\mathrm{PA}(\%)$ & 96.6 & 75.4 & 93.3 & 99.9 & 82.6 \\
\hline & OA(\%) & \multicolumn{5}{|c|}{89.0} \\
\hline
\end{tabular}


TO
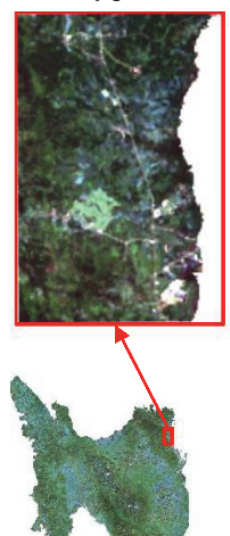

a

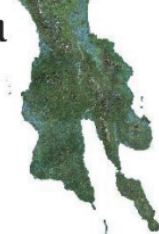

T1
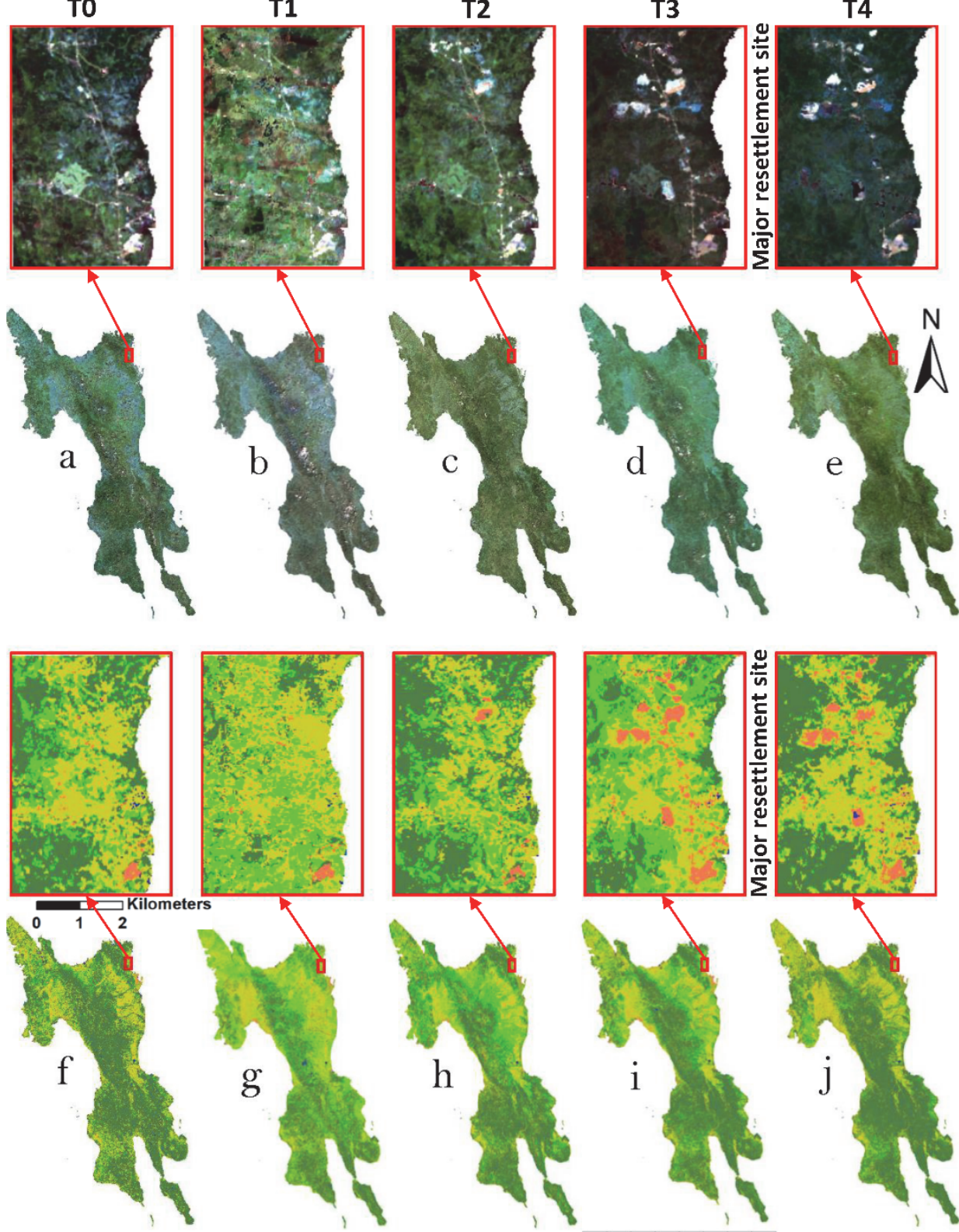

h

g

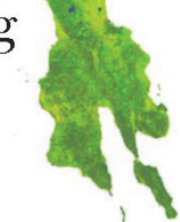

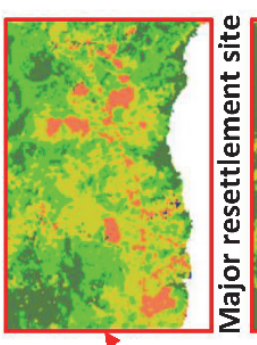
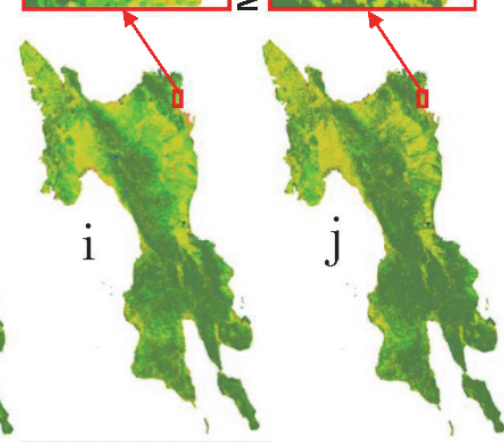

120 Kilometers

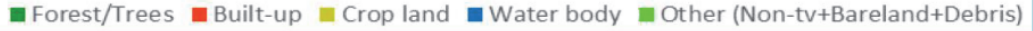

Figure 6.3 (a-e) Landsat image composites acquired over Leyte island for T0-T4 times, respectively, $(f-j)$ land cover classification maps produced with GEE for T0T4 times, respectively. Red rectangles show the status of the relocation site (northern part of Tacloban) for T0-T4 times. Non-tv-Non-tree vegetation. 
Figure 6.4 shows the land cover class change trends extracted from the T0 to T4 images. Typhoon Haiyan clearly had a significant impact on the forest/trees and built-up areas, reducing their coverage by about $40 \%$ and $30 \%$ from T0 to T1 images, respectively. The increase of the other class from T0 to T1 is mostly due to the debris and rubble caused by Haiyan and denuded trees, which led to an increase in non-tree vegetation coverage even in (formerly) forested areas. The storm surge during the typhoon resulted in the increase in water bodies in the T1 image. Monitoring three years after Typhoon Haiyan for Leyte island demonstrates that most of the land cover changes returned to their pre-disaster situation. However, there is an increase in the built-up class that is due to the construction of resettlement sites (as safe zones) mostly around the eastern coastal cities (e.g., Tacloban city-Figure 6.3).

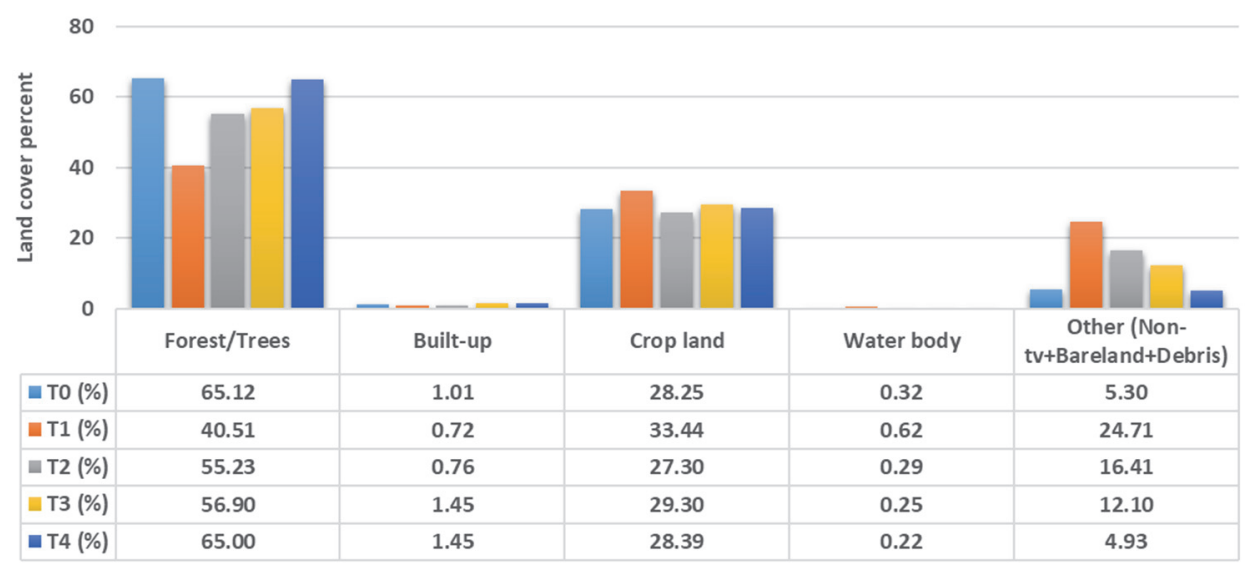

Figure 6.4 Per class land cover percentage for Leyte island for T0-T4. Non-tv-Non-tree vegetation.

Figure 6.5 shows the damage and recovery maps for the post-Typhoon Haiyan situation generated at the municipality level for Leyte island. The damage map was produced based on the change analysis of the land cover classes for the T0 and T1 times, and recovery maps (RC) (i.e., RC1, RC2, and RC3) were produced based on change analysis of the damage map and the land cover classes of $\mathrm{T} 2, \mathrm{~T} 3$, and $\mathrm{T} 4$ times, respectively. 


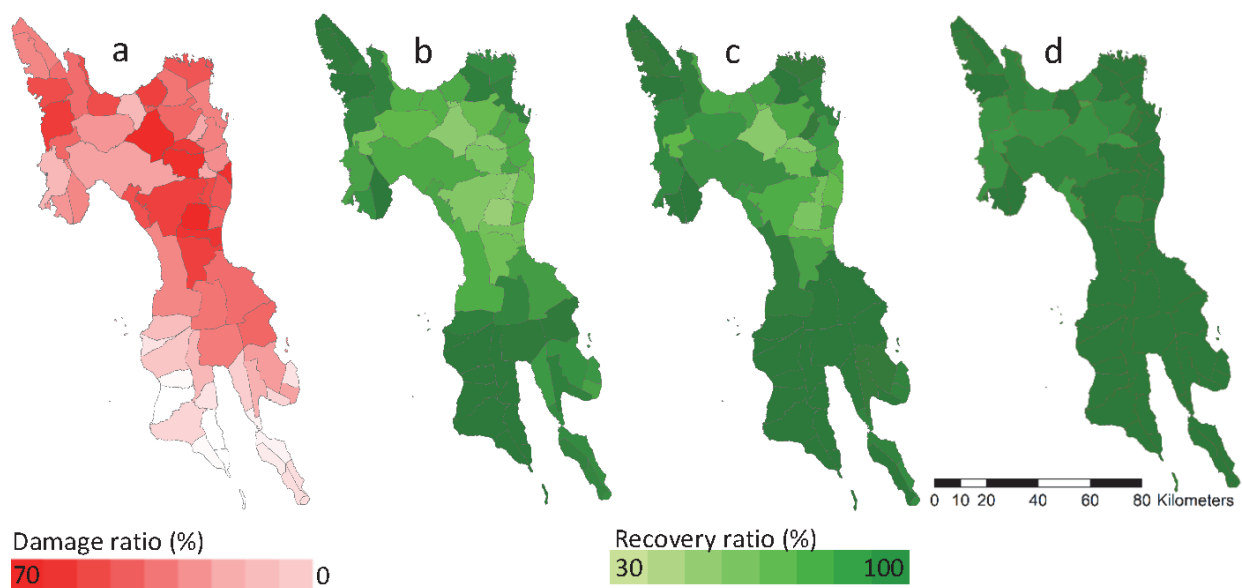

Figure 6.5 (a) Municipality level damage map for T1 and (b-d) the recovery maps for T2-T4 times after Typhoon Haiyan for Leyte island, respectively.

In Figure 6.5, the darker red color shows more damage while, for the recovery maps, the darker green color shows a higher level of recovery. The most damaged/impacted municipalities are located in the center and northern part of the island. However, since Haiyan passed through the northern part of the island, the municipalities in the southern parts have the lowest damage ratios, close to zero. The most affected land cover classes were the forest/trees and built-up classes (Figure 6.4). Consequently, the municipalities with more forest/trees and built-up coverage compared to other land cover types have higher damage ratios. The change in the recovery maps (from RC1 to RC3) shows that the municipalities with more forest/tree coverage recovered more slowly than the others, and eventually, after a period of three years, most of the forest trees had recovered (RC3) (Figure 6.5d). Moreover, the results reported in a previous study (Sheykhmousa et al. 2019) show almost the same pattern of recovery in terms of change in land cover classes at a small scale (i.e., Tacloban) in the post-Haiyan time. The only difference is in built-up class, where our study shows growth in built-up areas three years after Haiyan, while they reported that Tacloban city returned to almost the same level of built-up coverage four years after the disaster. This is mainly because their focus was on central urban areas of Tacloban, not including the northern part of Tacloban in their analysis, in which the primary resettlement sites for the period after Haiyan were built (Figure 6.3).

\subsection{Discussion and Conclusions}

The aim of this study was to assess the suitability of GEE for post-disaster recovery assessment for a large-scale region after Typhoon Haiyan, which hit Leyte island in the Philippines in 2013. We proposed an approach to monitor and quantify the recovery process using GEE, in particular with Landsat 
images, based on land cover classification and subsequent change analysis, and then producing municipality-based damage and recovery maps. The land cover classification results show the robustness of the employed approach, including image composite generation and Random Forest-based image classification. Furthermore, the change analysis and generated recovery maps clearly illustrate the damage and recovery processes during the selected time epochs, i.e., three time-steps for recovery assessment for three years after Haiyan. However, among the big geo dataset available in the platform and considering the time of Typhoon Haiyan (i.e., November 2013), we only found Landsat images suitable for this study. Hence, due to the limited spatial resolution of the data, detailed recovery analysis was not possible (e.g., extracting more land-use classes, such as building use and crop types). As also concluded by Sheykhmousa et al. (2019), land cover maps can provide the main information for decision-makers to have an overview of the damage and reconstruction/recovery processes, which are critical in the early stages of recovery. However, a comprehensive recovery assessment needs more details that can be obtained using land use maps extracted from very high-resolution images. Little work has been done to date in damage and recovery mapping of the selected area, and none of this previous research investigated the entire Leyte island. In addition, the available studies that focused on some selected small areas were based on very high-resolution images with different class definitions. Hence, the validation is only based on the accuracy assessment of the land cover classification results. However, since GEE also allows to upload user images for processing, it might be used to upload a very high-resolution image for validation purposes.

Moreover, the change in types of crops and trees, or any detail about the recovered trees, can provide more information about the recovery process. For example, it is essential from the environmental and ecological points of view to understand whether the same trees that were denuded by the typhoon recovered, or whether the trees detected were newly planted after the disaster. More detailed information on the age of the trees can provide significant additional insights into the post-disaster recovery process.

In conclusion, further studies are needed to investigate the potential of the more recently available higher resolution optical and SAR images (Sentinel 1 and 2 images) for most of the case studies (i.e., disasters) that occurred after those platforms became operational. In addition, SAR data have been shown to be a useful tool for extracting land cover and use classes such as distinguishing different crop and tree types (De Alban et al. 2018). Hence, using higher resolution images and SAR data available in GEE will allow the researchers to detect damage to the crops, contribute to detecting changes in tree types, and extract more details for urban areas after a disaster (Bell et al. 2019; Dadhich et al. 2019; Phan et al. 2019). 
Computer vision and image processing/classification methods deal with the actual image-based pixel values and try to classify them to achieve the best accuracies. However, in post-disaster remote sensing images, especially in water-related disasters, seeing actual damage and visually distinguishing damaged and intact areas is nearly impossible in some cases, as some intact areas are often buried under debris. Hence, assuming the debris area as the damaged class is not entirely correct, there is a need for further studies to identify the debris types (e.g., (Ghaffarian and Kerle 2019b)) or use proxies for distinguishing between damaged and other classes (Sheykhmousa et al. 2019) instead of only using direct image classification methods.

Evaluating the recovery process with more details (e.g., building use detection, crop type classification) and several time epochs at such a large scale, and providing municipality level recovery scores similar to those proposed in our study, can be further used for per-municipality resilience assessment at large scales, assuming that the speed of recovery is a proxy for evaluating resilience. However, it has been shown that this assumption is not correct for all cases, and further investigation is needed to clearly determine the relationship between the speed of the post-disaster recovery and resilience (Kerle et al. 2019a).

Although the Random Forest-based image classification produced a high accuracy rate, using more advanced methods (i.e., deep learning-based approaches) within the Google Earth API could help to improve the accuracy and would allow executing more advanced image classification and object detection tasks.

\section{Supplementary Materials}

Supplementary materials can be accessed at: https://www.mdpi.com/2076$3417 / 10 / 13 / 4574 / s 1$.

\subsection{References of Chapter 6}

Abdi, A. M., 2019. Land cover and land use classification performance of machine learning algorithms in a boreal landscape using Sentinel-2 data. GIScience \& Remote Sensing, 1-20.

Belgiu, M., Drăguţ, L., 2016. Random forest in remote sensing: A review of applications and future directions. ISPRS Journal of Photogrammetry and Remote Sensing, 114, 24-31.

Bell, J., Gebremichael, E., Molthan, A., Schultz, L., Meyer, F., Shrestha, S., 2019. Synthetic Aperture Radar and optical remote sensing of crop damage attributed to severe weather in the central United States. Paper presented at the IGARSS 2019, Yokohama, Japan, . 
Brown, D., S., P., J., B., 2010. Disaster recovery indicators: Guidlines for monitoring and evaluation. University of Cambridge: Cambridge, UK: CURBE, Cambridge University for Risk in the Built Environment.

Brown, D., Saito, K., Liu, M., Spence, R., So, E., Ramage, M., 2011. The use of remotely sensed data and ground survey tools to assess damage and monitor early recovery following the 12.5.2008 Wenchuan earthquake in China. Bulletin of Earthquake Engineering, 10(3), 741-764.

Burton, C., Mitchell, J. T., Cutter, S. L., 2011. Evaluating post-Katrina recovery in Mississippi using repeat photography. Disasters, 35(3), 488-509.

Canty, M. J., Nielsen, A. A., Conradsen, K., Skriver, H., 2019. Statistical Analysis of Changes in Sentinel-1 Time Series on the Google Earth Engine. Remote Sensing, 12(1), 46.

Chen, T., Guestrin, C., 2016. XGBoost: A Scalable Tree Boosting System. Paper presented at the Proceedings of the 22nd ACM SIGKDD International Conference on Knowledge Discovery and Data Mining, San Francisco, California, USA.

Contreras, D., Blaschke, T., Tiede, D., Jilge, M., 2016. Monitoring recovery after earthquakes through the integration of remote sensing, GIS, and ground observations: the case of L'Aquila (Italy). Cartography and Geographic Information Science, 43(2), 115-133.

Costa Viera, A., Kerle, N., 2014. Utility of geo-informatics for disaster risk management: Linking structural damage assessment, recovery and resilience. University of Twente, The Netherlands.

CRED. 2018. Economic losses, poverty and disasters 1998-2017.

Crowley, M. A., Cardille, J. A., White, J. C., Wulder, M. A., 2019. Generating intra-year metrics of wildfire progression using multiple open-access satellite data streams. Remote Sensing of Environment, 232, 111295.

Dadhich, G., Miyazaki, H., Babel, M., 2019. Applications of Sentinel-1 Synthetic Aperture Radar imagery for floods damage assessment: A case study of Nakhon Si Thammarat, Thailand. ISPRS - International Archives of the Photogrammetry, Remote Sensing and Spatial Information Sciences, 4213, 1927-1931.

De Alban, J. D. T., Connette, G. M., Oswald, P., Webb, E. L., 2018. Combined Landsat and L-Band SAR Data Improves Land Cover Classification and Change Detection in Dynamic Tropical Landscapes. Remote Sensing, 10(2), 306.

Duarte, D., Nex, F., Kerle, N., Vosselman, G., 2018. Multi-resolution feature fusion for image classification of building damages with convolutional neural networks. Remote Sensing, 10(10), 1636.

Ebert, A., Kerle, N., Stein, A., 2009. Urban social vulnerability assessment with physical proxies and spatial metrics derived from air- and spaceborne imagery and GIS data. Natural Hazards, 48(2), 275-294.

Fernández-Delgado, M., Cernadas, E., Barro, S., Amorim, D., 2014. Do we need hundreds of classifiers to solve real world classification problems? (Vol. 15): JMLR.org.

Gao, B.-C., 1996. NDWI-A normalized difference water index for remote sensing of vegetation liquid water from space. Remote Sensing of Environment, 58(3), 257-266.

Ge, Y., Hu, S., Ren, Z., Jia, Y., Wang, J., Liu, M., Zhang, D., Zhao, W., Luo, Y., $\mathrm{Fu}, \mathrm{Y} ., \mathrm{Bai}, \mathrm{H}_{.}$, Chen, Y., 2019. Mapping annual land use changes in 
China's poverty-stricken areas from 2013 to 2018. Remote Sensing of Environment, 232, 111285.

Georganos, S., Grippa, T., Vanhuysse, S., Lennert, M., Shimoni, M., Wolff, E., 2018. Very High Resolution Object-Based Land Use-Land Cover Urban Classification Using Extreme Gradient Boosting. IEEE Geoscience and Remote Sensing Letters, 15(4), 607-611.

Ghaffarian, S., Ghaffarian, S., 2014a. Automatic building detection based on Purposive FastICA (PFICA) algorithm using monocular high resolution Google Earth images. ISPRS Journal of Photogrammetry and Remote Sensing, 97, 152-159.

Ghaffarian, S., Ghaffarian, S., 2014b. Automatic building detection based on supervised classification using high resolution Google Earth images. Paper presented at the The International Archives of Photogrammetry, Remote Sensing and Spatial Information Sciences, 40, 101.

Ghaffarian, S., Kerle, N., 2019a. Post-disaster recovery assessment using multi-temporal satellite images with a deep learning approach. Paper presented at the 39th Earsel conference, Salzburg, Austria.

Ghaffarian, S., Kerle, N., 2019b. Towards post-disaster debris identification for precise damage and recovery assessments from UAV and satellite images. Int. Arch. Photogramm. Remote Sens. Spatial Inf. Sci., XLII-2/W13, 297302.

Ghaffarian, S., Kerle, N., Filatova, T., 2018. Remote Sensing-Based Proxies for Urban Disaster Risk Management and Resilience: A Review. Remote Sensing, 10(11), 1760.

Ghaffarian, S., Kerle, N., Pasolli, E., Jokar Arsanjani, J., 2019. Post-Disaster Building Database Updating Using Automated Deep Learning: An Integration of Pre-Disaster OpenStreetMap and Multi-Temporal Satellite Data. Remote Sensing, 11(20), 2427.

Gislason, P. O., Benediktsson, J. A., Sveinsson, J. R., 2006. Random Forests for land cover classification. Pattern Recognition Letters, 27(4), 294-300.

Gorelick, N., Hancher, M., Dixon, M., Ilyushchenko, S., Thau, D., Moore, R., 2017. Google Earth Engine: Planetary-scale geospatial analysis for everyone. Remote Sensing of Environment, 202, 18-27.

Griffiths, P., Linden, S. V. D., Kuemmerle, T., Hostert, P., 2013. A Pixel-Based Landsat Compositing Algorithm for Large Area Land Cover Mapping. Ieee Journal of Selected Topics in Applied Earth Observations and Remote Sensing, 6(5), 2088-2101.

Ham, J., Yangchi, C., Crawford, M. M., Ghosh, J., 2005. Investigation of the random forest framework for classification of hyperspectral data. IEEE Transactions on Geoscience and Remote Sensing, 43(3), 492-501.

Hansen, M. C., Loveland, T. R., 2012. A review of large area monitoring of land cover change using Landsat data. Remote Sensing of Environment, 122, 66-74.

Harb, M. M., De Vecchi, D., Dell'acqua, F., 2015. Phisical Vulnerability Proxies from Remotes Sensing: Reviewing, Implementing and Disseminating Selected Techniques. IEEE Geoscience and Remote Sensing Magazine, 3(1), 20-33.

Hoshi, T., Murao, O., Yoshino, K., Yamazaki, F., Estrada, M., 2014. Postdisaster urban recovery monitoring in pisco after the 2007 peru earthquake using satellite image. . J. Disaster Res., 9, 1059-1068. 
Huete, A., Didan, K., Miura, T., Rodriguez, E. P., Gao, X., Ferreira, L. G., 2002. Overview of the radiometric and biophysical performance of the MODIS vegetation indices. Remote Sensing of Environment, 83(1), 195-213.

Huete, A. R., Liu, H. Q., Batchily, K., Van Leeuwen, W., 1997. A comparison of vegetation indices over a global set of TM images for EOS-MODIS. Remote Sensing of Environment, 59(3), 440-451.

Jurgens, C., 1997. The modified normalized difference vegetation index (mNDVI) a new index to determine frost damages in agriculture based on Landsat TM data. International Journal of Remote Sensing, 18(17), 35833594.

Kelley, L. C., Pitcher, L., Bacon, C., 2018. Using Google Earth Engine to Map Complex Shade-Grown Coffee Landscapes in Northern Nicaragua. Remote Sensing, 10(6), 952.

Kerle, N., 2016. Disasters: Risk assessment, management, and post-disaster studies using remote sensing Remote sensing of water resources, disasters, and urban studies: Taylor \& Francis Group, LLC.

Kerle, N., Ghaffarian, S., Nawrotzki, R., Leppert, G., Lech, M., 2019a. Evaluating Resilience-Centered Development Interventions with Remote Sensing. Remote Sensing, 11(21), 2511.

Kerle, N., Nex, F., Gerke, M., Duarte, D., Vetrivel, A., 2019b. UAV-based structural damage mapping: A review. ISPRS International Journal of GeoInformation, 9(1), 14.

Kumar, L., Mutanga, O., 2018. Google Earth Engine Applications Since Inception: Usage, Trends, and Potential. Remote Sensing, 10(10), 1509.

Liu, C.-C., Shieh, M.-C., Ke, M.-S., Wang, K.-H., 2018. Flood Prevention and Emergency Response System Powered by Google Earth Engine. Remote Sensing, 10(8), 1283.

Mahdianpari, M., Salehi, B., Mohammadimanesh, F., Homayouni, S., Gill, E., 2018. The First Wetland Inventory Map of Newfoundland at a Spatial Resolution of $10 \mathrm{~m}$ Using Sentinel-1 and Sentinel-2 Data on the Google Earth Engine Cloud Computing Platform. Remote Sensing, 11(1), 43.

Marsett, R. C., Qi, J., Heilman, P., Biedenbender, S. H., Carolyn Watson, M., Amer, S., Weltz, M., Goodrich, D., Marsett, R., 2006. Remote Sensing for Grassland Management in the Arid Southwest. Rangeland Ecology \& Management, 59(5), 530-540.

NDRRMC. 2014. Final reports for effects of Typhoon Yolanda (Haiyan). Retrieved from http://www.ndrrmc.gov.ph/attachments/article/1329/FINAL_REPORT_re _Effects_of_Typhoon_YOLANDA_HAIYAN_06-09NOV2013.pdf

Nex, F., Duarte, D., Steenbeek, A., Kerle, N., 2019a. Towards real-time building damage mapping with low-cost UAV solutions. Remote Sensing, 11(3), 287.

Nex, F., Duarte, D., Tonolo, F. G., Kerle, N., 2019b. Structural Building Damage Detection with Deep Learning: Assessment of a State-of-the-Art CNN in Operational Conditions. Remote Sensing, 11(23), 2765.

Oliphant, A. J., Thenkabail, P. S., Teluguntla, P., Xiong, J., Gumma, M. K., Congalton, R. G., Yadav, K., 2019. Mapping cropland extent of Southeast and Northeast Asia using multi-year time-series Landsat 30-m data using a random forest classifier on the Google Earth Engine Cloud. International Journal of Applied Earth Observation and Geoinformation, 81, 110-124. 
Phan, A., N. Ha, D., D. Man, C., T. Nguyen, T., Q. Bui, H., T. N. Nguyen, T., 2019. Rapid Assessment of Flood Inundation and Damaged Rice Area in Red River Delta from Sentinel 1A Imagery. Remote Sensing, 11(17), 2034.

Reductions., G. D. I., 2019. Available online: https://developers.google.com/earthengine/reducers_image_collection.

Sazib, N., Mladenova, I., Bolten, J., 2018. Leveraging the Google Earth Engine for Drought Assessment Using Global Soil Moisture Data. Remote Sensing, 10(8), 1265.

Shelestov, A., Lavreniuk, M., Kussul, N., Novikov, A., Skakun, S., 2017. Exploring Google Earth Engine Platform for Big Data Processing: Classification of Multi-Temporal Satellite Imagery for Crop Mapping. Frontiers in Earth Science, 5(17).

Sheykhmousa, M., Kerle, N., Kuffer, M., Ghaffarian, S., 2019. Post-disaster recovery assessment with machine learning-derived land cover and land use information. Remote Sensing, 11(10), 1174.

Sidhu, N., Pebesma, E., Câmara, G., 2018. Using Google Earth Engine to detect land cover change: Singapore as a use case. European Journal of Remote Sensing, 51(1), 486-500.

Stromann, O., Nascetti, A., Yousif, O., Ban, Y., 2019. Dimensionality Reduction and Feature Selection for Object-Based Land Cover Classification based on Sentinel-1 and Sentinel-2 Time Series Using Google Earth Engine. Remote Sensing, 12(1), 76.

Stumpf, A., Kerle, N., 2011. Object-oriented mapping of landslides using Random Forests. Remote Sensing of Environment, 115(10), 2564-2577.

Sutton, P., Elvidge, C., Ghosh, T., 2007. Estimation of gross domestic product at sub-national scales using night-time satellite imagery. Int. J. Ecol. Econ. Stat., 8, 5-21.

Teluguntla, P., Thenkabail, P. S., Oliphant, A., Xiong, J., Gumma, M. K., Congalton, R. G., Yadav, K., Huete, A., 2018. A 30-m landsat-derived cropland extent product of Australia and China using random forest machine learning algorithm on Google Earth Engine cloud computing platform. ISPRS Journal of Photogrammetry and Remote Sensing, 144, 325-340.

Torbick, N., Ledoux, L., Salas, W., Zhao, M., 2016. Regional Mapping of Plantation Extent Using Multisensor Imagery. Remote Sensing, 8(3), 236.

Tucker, C. J., 1979. Red and photographic infrared linear combinations for monitoring vegetation. Remote Sensing of Environment, 8(2), 127-150.

UNISDR. 2015, 14-18 March. Sendai framework for disaster risk reduction 2015 - 2030. Paper presented at the Third World Conf. Disaster Risk Reduction, Sendai, Japan.

Van Deventer, A. P., Ward, A. D., Gowda, P. H., Lyon, J. G., 1997. Using Thematic Mapper data to identify contrasting soil plains and tillage practices. . Photogramm. Eng. Remote Sens., 63, 87-93.

Vetrivel, A., Gerke, M., Kerle, N., Vosselman, G., 2016a. Identification of structurally damaged areas in airborne oblique images using a visual-bagof-words approach. Remote Sensing, 8(3), 231.

Vetrivel, A., Kerle, N., Gerke, M., Nex, F., Vosselman, G., 2016b. Towards automated satellite image segmentation and classification for assessing disaster damage using data specific features with incremental learning. Paper presented at the GEOBIA 2016, Enschede, The Netherlands. 
White, J. C., Wulder, M. A., Hobart, G. W., Luther, J. E., Hermosilla, T., Griffiths, P., Coops, N. C., Hall, R. J., Hostert, P., Dyk, A., Guindon, L., 2014. Pixel-Based Image Compositing for Large-Area Dense Time Series Applications and Science. Canadian Journal of Remote Sensing, 40(3), 192-212.

Wu, Q., Lane, C. R., Li, X., Zhao, K., Zhou, Y., Clinton, N., Devries, B., Golden, H. E., Lang, M. W., 2019. Integrating LiDAR data and multi-temporal aerial imagery to map wetland inundation dynamics using Google Earth Engine. Remote Sensing of Environment, 228, 1-13.

Wulder, M. A., Masek, J. G., Cohen, W. B., Loveland, T. R., Woodcock, C. E., 2012. Opening the archive: How free data has enabled the science and monitoring promise of Landsat. Remote Sensing of Environment, 122, 210.

Xiao, X., Boles, S., Frolking, S., Salas, W., Moore, B., Li, C., He, L., Zhao, R., 2002. Landscape-scale characterization of cropland in China using Vegetation and Landsat TM images. International Journal of Remote Sensing, 23(18), 3579-3594.

Xie, Z., Phinn, S. R., Game, E. T., Pannell, D. J., Hobbs, R. J., Briggs, P. R., Mcdonald-Madden, E., 2019. Using Landsat observations (1988-2017) and Google Earth Engine to detect vegetation cover changes in rangelands - A first step towards identifying degraded lands for conservation. Remote Sensing of Environment, 232, 111317.

Xiong, J., Thenkabail, P. S., Gumma, M. K., Teluguntla, P., Poehnelt, J., Congalton, R. G., Yadav, K., Thau, D., 2017. Automated cropland mapping of continental Africa using Google Earth Engine cloud computing. ISPRS Journal of Photogrammetry and Remote Sensing, 126, 225-244.

Zhou, B., Okin, G. S., Zhang, J., 2020. Leveraging Google Earth Engine (GEE) and machine learning algorithms to incorporate in situ measurement from different times for rangelands monitoring. Remote Sensing of Environment, 236, 111521. 


\section{Chapter 7 - Agent-based modelling of post- disaster recovery with remote sensing data ${ }^{6}$}

6 This chapter is based on:

- Ghaffarian, S.; Roy, D; Filatova, T.; Kerle, N., 2020. Agent-based modelling of postdisaster recovery with remote sensing data. International Journal of Disaster Risk Reduction. (Under review) 


\section{Abstract}

Disaster risk management, and post-disaster recovery (PDR) in particular, become increasingly important to assure resilient development. Yet, PDR is the most poorly understood phase of the disaster management cycle and can take years or even decades. The physical aspects of the recovery are relatively easy to monitor and evaluate using, e.g. geospatial remote sensing data compared to functional assessments that include social and economic processes. Therefore, there is a need to explore the impacts of different dimensions of the recovery, including individual behaviour and their interactions with socioeconomic institutions. In this study, we develop an agent-based model to simulate and explore the PDR process in urban areas of Tacloban, the Philippines devastated by Typhoon Haiyan in 2013. Formal and informal (slum) sector households are differentiated in the model to explore their resilience and different recovery patterns. Machine learning-derived land use maps are extracted from remote sensing images for pre- and post-disaster and are used to provide information on physical recovery. We use the empirical model to evaluate two realistic policy scenarios: the construction of relocation sites after a disaster and the investments in improving employment options. We find that the speed of the recovery of the slum dwellers is higher than formal sector households due to the quick reconstruction of slums and the availability of lowincome jobs in the first months after the disaster. Finally, the results reveal that the households' commuting distance to their workplaces is one of the critical factors in households' decision to relocate after a disaster. 


\subsection{Introduction}

Annually natural disasters take a high toll in terms of assets and people globally. Between 1998-2017 the number of affected people increased from 4 to more than 5.7 billion people (CRED 2015, 2018), imposing US\$ 520 billion of damages in real annual global economic costs (The World Bank 2017; CRED 2018). This escalation is due to the accelerated urbanization and the increase in the number and severity of natural disasters triggered by climate change. However, these losses are not equally distributed across countries. Low-income countries incurred substantially higher Gross Domestic Product (GDP) losses over the past 20 years due to natural disasters when compared to high-income countries (CRED 2018). The rapid increase in disaster severity and frequency and associated damage calls for effective disaster risk reduction and management strategies at different scales, including individual actions.

One of the main phases of the disaster risk management cycle is the recovery phase, which usually starts after the operations for the response phase have concluded, and whose effectiveness has a significant effect on the final disaster cost. Post-disaster recovery (PDR) is known as a process to rebuild the community to normal conditions/functioning level, i.e. the same as before the disaster. However, it is essential to use the recovery process as an opportunity to rebuild the affected area better as per the Sendai Framework (Unisdr 2015). Improving disaster preparedness through the building back better concept in the recovery phase is the Framework's priority, resulting in more resilient and sustainable communities (Brundiers and Eakin 2018; UNISDR 2015). This becomes vital where the vicious cycle of disasters weakens affected areas, setting them up for rapid follow-on disasters (Alcayna T et al. 2016; Bank 2018). Nevertheless, recovery is a dynamic process that varies in duration and quality (Brown et al. 2010). Furthermore, a holistic recovery process goes beyond a physical recovery of infrastructure and includes a re-establishment of social, economic, and natural environmental processes (Mcdem 2005). Hence, it involves multiple sectors, governmental departments, policymakers, and households working together. This makes recovery a complex process. Therefore, there is a need for monitoring and providing tools for decisionmakers to collect information about the ongoing PDR process and understand the effect of different scenarios in dynamics. Evaluating the PDR can give valuable information to the decision-makers regarding the current stage of the process, useful for monitoring and comparison with the envisioned recovery plans (Ghaffarian and Kerle 2019a; Ghaffarian et al. 2018; Kerle et al. 2019a; Sheykhmousa et al. 2019).

Increasingly, there is a need to understand and explore the impacts of different dimensions of recovery, including the behavior of individual actors and their interactions with socio-economic institutions. Computer-based simulations 
such as agent-based models (ABMs) permit to explore the dynamics of the recovery process from the bottom up. ABMs are computational models of societies where different actors - households, firms, farmers, governments act, learn, interact, and co-evolve with their environment (Fiedrich and Burghardt 2007). In an ABM, agents (decision-making entities) interact with each other and their environments to decide and act based on defined rules for their behaviour in a specific situation such as a PDR (Tesfatsion 2005; Wooldridge 2009). ABMs, which simulate paths between equilibria and emergence of new post-disaster states, generate a wider range of nonlinear behaviour than conventional models that focus on the recovery as the 'return to normality'. Therefore, it constitutes an opportunity for policy-makers to test different policy scenarios in an artificial simulation environment and explore their consequences (Farmer and Foley 2009). Accordingly, policy and decisionmakers can take advantage of the simulation outcomes to steer the recovery process.

ABMs are used frequently in the disaster management domain (An 2012; Burger et al. 2019; Filatova 2014; Filatova et al. 2011; Mehvar et al. 2019; Watts et al. 2019) such as flood management (Abebe et al. 2019; Dawson et al. 2011), disaster evacuation modeling (Chen and Zhan 2008; Wang et al. 2016), coastal adaptation (Mcnamara and Keeler 2013), disaster impact assessment (Grinberger and Felsenstein 2016; Markhvida et al. 2020; Mehvar et al. 2019), recovery modeling (Eid and El-Adaway 2015; Sun et al. 2019) and resilience assessment (Boston et al. 2014; Sun et al. 2019; Taylor et al. 2015). The response phase management and evacuation after a disaster have been extensively addressed in the literature (Burger et al. 2019; Chen and Zhan 2008; Markhvida et al. 2020; Wang et al. 2016). However, PDR management as a long-term process has not yet been sufficiently studied to extract the influential factors and their impacts on this process. Besides, formal and informal (slum) sector households have not been studied as separate groups for recovery modelling and resilience assessment in developing countries. Furthermore, the potential of the alternative data sources for survey data, in particular remote sensing (RS) data, for ABMs have not been sufficiently studied.

RS as a rapid and effective tool to collect geospatial data has been used for different purposes in the DRM domain (Ghaffarian and Kerle 2019b; Ghaffarian et al. 2018; Kerle et al. 2019a; Kerle et al. 2019b; Sheykhmousa et al. 2019). Also, recent advances in machine learning/computer vision methods and computer hardware have increased the accuracy and speed of the semi/automatic approaches in the extraction of information from RS data (Duarte et al. 2018; Ghaffarian and Kerle 2019a; Ghaffarian et al. 2019; Vetrivel et al. 2015). These improvements make RS a reliable alternative for fieldwork-based data collection approaches, particularly when there is a need 
for Earth surface-related information. RS data, especially satellite images, are of particular importance in data-scarce situations. Spatial data from standard GIS layers have been employed in ABMs; however, RS was not been used before as the primary source for a PDR ABM, especially in the Global South DRM research.

This article presents an ABM to explore the post-disaster recovery process (the PDR ABM). We apply the model to Tacloban, the Philippines, which hit by super Typhoon Haiyan in 2013. The innovative contribution of this article is four-fold. Firstly, RS data are employed as the main data source to initialize the PDR ABM and the reconstruction of the built-up area in the recovery process. RS data can be used to monitor the recovery process directly by extracting physical aspects of the process (Ghaffarian et al. 2019; Sheykhmousa et al. 2019) or indirectly using proxies to conduct functional recovery assessments (Ghaffarian et al. 2018; Sheykhmousa et al. 2019). In our study, RS data are used to extract multi-temporal land use maps, including slum and formal building information, using advanced machine learning methods. And Secondly, we differentiate between the behaviour of formal and informal (slum) sector households, which follow different decision-making strategies in the recovery process. The outputs of the model and the multi-temporal utility satisfaction can serve to evaluate the disaster resilience of these target groups. This also allows us to go beyond the physical aspects and understand/explore the socioeconomic factors of the PDR dynamics. Thirdly, the spatial distribution of the households utility satisfaction is visualized and overlaid with the highresolution satellite images that add the capability of exploring the spatial recovery patterns. Fourthly, we use the developed model to run two realistic policy scenarios: the construction of relocation sites after a disaster and the investments in improving employment options. In what follows we describe the methodology and the case-study, present the simulation results and discuss them in the context of disaster risk and resilience management.

\subsection{Methods}

\subsubsection{Case-study and data}

Tacloban city, located in the Eastern Visayas, is the biggest city and the economic centre of the Leyte region in the Philippines (Figure 7.1). The city has a population of approximately 250.000, with an economy largely focused on commerce, agriculture, fishing, industry (mostly palm oil factories), tourism, and trade. There are formal and informal (slums, mostly stretched along the coast) neighbourhoods in the city, showing the socio-economic diversity of the population. On the $8^{\text {th }}$ of November 2013, Tacloban was hit by Typhoon Haiyan (locally known as Typhoon Yolanda), which was one of the strongest typhoons ever to make a landfall worldwide (Mori et al. 2014). The 
occurrence of a storm surge of up to $5 \mathrm{~m}$ led to an official fatality number of 6201 for the city, mostly killing people who lived in the coastal neighbourhoods (Ching Pk et al. 2015).

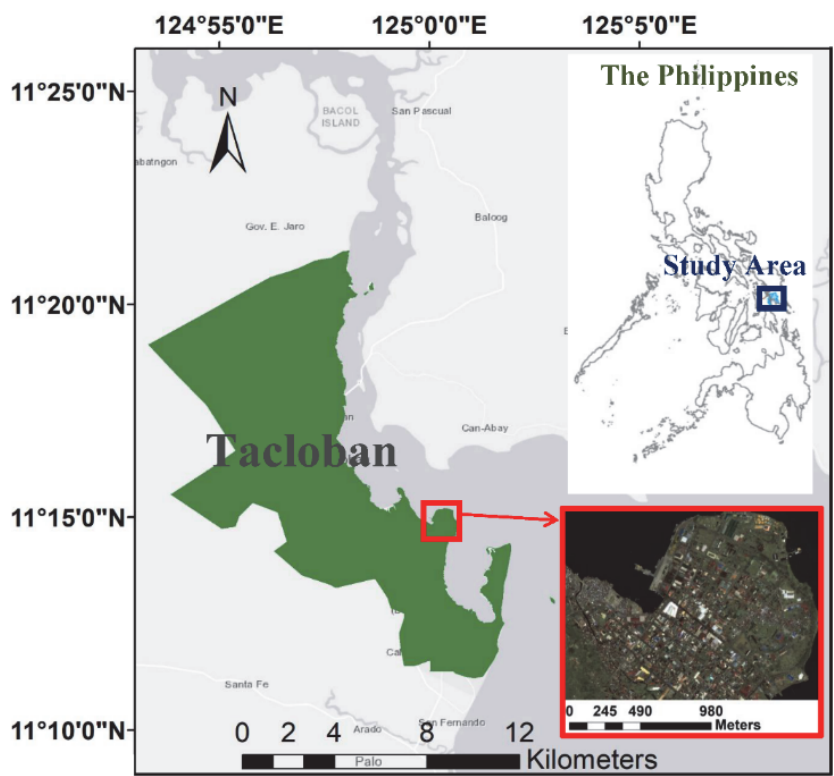

Figure 7.1 The overview of Tacloban, the Philippines, and the satellite image for the modelled urban area acquired before Haiyan.

Using the RS data (i.e. high-resolution satellite images) we can extract the built-up areas, including slum and formal buildings, of the city before the disaster and trace the recovery process in the first years following Haiyan. To explore the social processes driving this recovery, we developed the PDR ABM, parameterized with the maps extracted from the RS data. Advanced machine learning methods (Kerle et al. 2019a; Sheykhmousa et al. 2019) were employed to extract the land use maps from high-resolution satellite images (for the pre-disaster situation, as well as three days, three months, and eight months after Haiyan) (Table 7.1). The land use raster maps of the region were converted to points with corresponding attributes as a GIS map of the urbanized region to be used in the model. Accordingly, informal and formal built-up areas were identified. Also, the buildings damaged by Haiyan and the reconstruction levels of the area during the recovery process for each month were extracted from the land use maps. According to the results reported by Sheykhmousa et al. (2019) on the reconstruction of the Tacloban area for four years after Haiyan and the best of our knowledge, we assumed that the modelled area had been fully reconstructed after five years. 
Table 7.1 Satellite images used in this study.

\begin{tabular}{ccccc}
\hline ID & Timeline & Acquired date & $\begin{array}{l}\text { Satellite } \\
\text { platform }\end{array}$ & Description \\
\hline T0 & Before Haiyan & $2013-03-17$ & WorldView-2 & Pansharpened images \\
T1 & Event time & $2013-11-11$ & WorldView-2 & with 0.5 m spatial \\
T2 & Post-Haiyan 1 & $2014-01-25$ & Pleiades & resolution \\
T3 & Post-Haiyan 2 & $2014-06-16$ & Pleiades &
\end{tabular}

Moreover, in 2015 we conducted extensive fieldwork in the area to understand how households made decisions and what impacted them. Specifically, we carried out interviews with key stakeholders, analyzed survey data (collected for the study (Kerle et al. 2019a)), and the demographic data collected from the Philippines Statistics Authority (e.g., employment rate, type of the economic activities before and after the disaster). The key persons, including authorities of the Tacloban city and a group of farmers and fishermen, were interviewed to understand the characteristics of the community, and how the disaster impacted them. Among others, the fieldwork revealed that immediately after Haiyan there was a relocation site developed in the northern part of Tacloban, aimed at becoming a safe area to move people out of danger zones, mostly slum dwellers. However, the relocation site was far from the city centre and the coast where most of the employment opportunities are located. We use the presence of the relocation site as well as the creation of additional employment opportunities as scenarios in the PDR ABM.

\subsubsection{Agent-based modelling of post-disaster recovery}

Related work: Scholars actively use ABMs to simulate and understand the recovery process after a disaster (Burger et al. 2019; Mishra et al. 2018). As one of the initial ABM-based studies in post-disaster recovery, Nejat and Damnjanovic (2012) developed a spatial-temporal ABM based on the dynamic homeowners' interactions with their neighbours in a post-disaster recovery situation. They showed that the discount factor (i.e., the weight of homeowners' utility from reconstruction) and the accuracy of the signals (i.e. the owners' future reconstruction property value) have impacts on the reconstruction of the houses. However, their model only focuses on housing recovery/reconstruction without including the individual personal factors/attributes. Afterwards, researchers tried to add individual personal behaviours to $A B M$ for different purposes and applications for the recovery process. Kanno et al. (2018) developed an ABM framework for the simulation of the post-disaster recovery in urban systems, with a final goal of disaster resilience assessment. In their $A B M$, they defined agents representing the behavior of the civil life, production industry, and infrastructures to understand their impact on the recovery and resilience. Their results showed that each of the subsystems has an impact on the resilience (i.e., the action or act of rebounding or springing back) of the urban systems with different 
coefficients/weights. In another study, tourist recovery strategies were studied using ABM after an earthquake in Jiuzhai Valley, China (in August 2017) (Fan et al. 2019). Their model provides a tool for managers to have an overall estimation of future tourist decline, as well as economic losses during the postearthquake recovery period. Coates et al. (2019) developed an ABM to assess the flood recovery and preparedness adaptation measures for small and medium-sized enterprises. They mainly studied physical and social adaptation factors and showed their combined significance effects in the adaptation of a key industrial area of the UK for a severe flooding scenario. In a different study, an ABM was developed to support sustainable disaster recovery by adding the environmental vulnerability to the model, and thus the decision-making process. The model was used to improve the community's welfare by reducing the vulnerability of the area to disasters and increasing the residents' needs/objective function (e.g. income and monthly distributed tax amount) (Eid and El-Adaway 2017). Current post-disaster recovery ABM simulates the entire community, ignoring decision traits that may vary among low and highincome households. In our case, formal and informal residents vary in their behavioural strategies, mostly due to their education, employment opportunities and economic level. In addition, the models usually utilize extensive survey-based information and ignore high-resolution RS information for modelling.

The PDR ABM: We developed a spatial ABM to simulate the decision-making process of individuals in a post-disaster recovery process in a city. The PDR model provides a tool to understand how households (living in informal or formal settlements) change jobs and locations to live after a disaster, driven by primary factors that influence their decisions during the reconstruction process. Appendix A provides a detailed description of the model according to the ODD + D protocol (Müller et al. 2013). Here we briefly outline the main agents, the rules guiding their choices and the overall flow of the model.

The main agents in the PDR ABM are households and buildings in an urban environment. There are two types of households: those residing in formal (FH) or informal (IH) urban areas that have different behaviours mostly due to their economic and education level. Further, households can be heterogeneous within each group, differentiated by income, education and workplace. Buildings in the modelled city can be for residential (formal or informal) and industrial use. Industrial buildings, important as they represent the location of mid- and high-income jobs, usually accessible only for FH with a higher level of education compared to IH. IH, which reside in slums, often have jobs in an informal sector, such as in fishing or as a seasonal labourer in the agricultural sector. 
Households agents aim to satisfy their utility by choosing where to live and what job to take. Currently, the PDR ABM is applied to several neighbourhoods in Tacloban and heavily relies on empirical data as explained in 2.1 and Appendix A. Households' utility is shaped by agents' social and economic needs (i.e. job, education, income, accessibility to the workplace). These factors help to assess how satisfied an agent is with living in a particular location, and having/lacking a job, the match between its income and education level, and the commute to its workplace. Though agents try bounded rationality: while they prefer an action that improves their utility, they are not searching for a global maximum. Such actions include: relocating their place of residence, changing a job, or both combined. The agent can change its job or location consulting its social network or by searching among available options individually. To define the social network and agent interactions in the PDR model we employ the theory of homophily (Mcpherson et al. 2001; Roy and Lees 2020), in which the social network is based on the degree of similarity (homophily) between two agents. To do so, we first determine the contact network and collect the information, then the agent selects through the available opportunities provided by the networks. In the PDR model, the degree of homophily is computed based on the similarity of the three agent attributes: job category/workplace, education, and income level (Eq. A.3). As a proxy for the accessibility analysis in the PDR model, we employed the distance to the workplace, which was computed from the RS data (please refer to Appendix A for details). Based on our interviews, we assume that IHs prefer to live near their workplaces, having therefore short daily commute.

The PDR model is initialized using the point-based land use data $(\mathrm{FH}, \mathrm{IH}$, and location of the industrial workplaces). In total, the agents are randomly assigned to 2131 informal residential and 1703 formal housing areas at initialization. The agents' state variables for individual IH and FH households are derived from the land use map of the Tacloban urban area, key interviews, census data, and survey data as described in Appendix (Table 7.2). The PDR model started from one month before Haiyan and evolved with a time step equal to one month. Hence, the PDR model started in September 2013 with a total of 11,502 agents.

During each step of the recovery phase in the PDR ABM, a damaged building may be reconstructed or a new one added to the residential building stock for agents to live in. The monthly increase of the reconstructed buildings was extracted separately for formal buildings and slums using the machine learning-derive land use maps from the satellite images (see 2.1). 


\subsection{Results and discussion}

Our PDR ABM simulates the post-disaster recovery to explore the recovery patterns of formal sector households and slum dwellers. Their differences affect decision making during the recovery process. We focus specifically on the effects of relocation site existence after a disaster and the dynamics of the employment rate in the model. In some post-disaster cases, such as in Tacloban, based on the hazard exposure and vulnerability measurements, governments decide to relocate people from high natural hazard risk to safer zones; however, this policy is not always successful. To model this policy scenario, we added a new residential area - built in the Tacloban case - as formal houses to the model that becomes accessible after the disaster, which is far from central urban areas. As a second policy scenario, we consider a boost in the job recovery commonly supported by NGOs in disaster-impacted areas. Having a job is key for individual welfare and socio-economic resilience in every society. Hence, we assess the impacts of changing employment rates on the recovery process and the resilience for both IH and FH by changing the employment rate while the other parameters are fixed.

The model was executed 30 times for each of the experiments under the same parameter combinations. We present the mean and standard deviation values of households' utility satisfaction across the $\mathbf{3 0}$ model runs. Since this metric is computed based on the socio-economic status of the households (e.g., income and education level) as well as the physical recovery rate (i.e., reconstruction of the buildings), it provides a holistic overview of the recovery status of the households (please refer to Appendix A. for more details). Socioeconomic assumptions at the micro-level of the households choices are based on the insights from our field work. The results of the model at the macro-level are validated by comparing them with the actual recovery patterns in the RS data. However, since RS data capture only the physical aspects of the recovery - damage and reconstruction of buildings - and not the utility satisfaction of individual households, a macro validation of this socio-economic dimension was not feasible given the available data.

\subsubsection{Post-disaster recovery patterns}

We implement the PDR model based on the available data and the information collected using both machine learning-derived information from RS images and census data. The PDR model results are employed to show and discuss the recovery patterns of the $\mathrm{IH}$ and $\mathrm{FH}$ in the urban area of Tacloban after Haiyan for the first 18 months after the disaster. Figure 7.2 shows the mean satisfaction of the $\mathrm{IH}$ and $\mathrm{FH}$ starting from before the disaster (step $=0$ ), and immediately after the disaster ( recovery process. Indeed, this figure illustrates the post-disaster recovery curve of the area in terms of the utility satisfaction of the households. The 
increasing speed of the IH satisfaction in the early recovery phase (in the first four months) is higher than the one with $\mathrm{FH}$, indicating that the $\mathrm{IH}$ is more resilient than the $\mathrm{FH}$ in terms of coming back to the same utility satisfaction levels. Yet, it does not necessarily demonstrate that IH is indeed more resilient since the original state was not, to begin with. In addition, the recovery rates of the $\mathrm{IH}$ and $\mathrm{FH}$ are $97 \%$ and $103 \%$, where above $100 \%$ shows the better mean utility satisfaction than pre-disaster situation, and thus, reaching the build-back-better goal. The speedy recovery of slum dwellers occurs due to two main reasons: (1) slums were reconstructed much faster than the formal buildings since it is easier to build a slum dwelling compared to a formal structure; (2) availability of low-income jobs is higher than high-income jobs in the early recovery phase, and the IH are more easily satisfied even with lower-income occupations due to their generally low education level. At the same time FHs prefer to have high-income jobs, given their education level. Yet, the large industries and factories - which most of the FHs prefer as a workplace offering higher-incomes - take more time to reconstruct after a disaster.

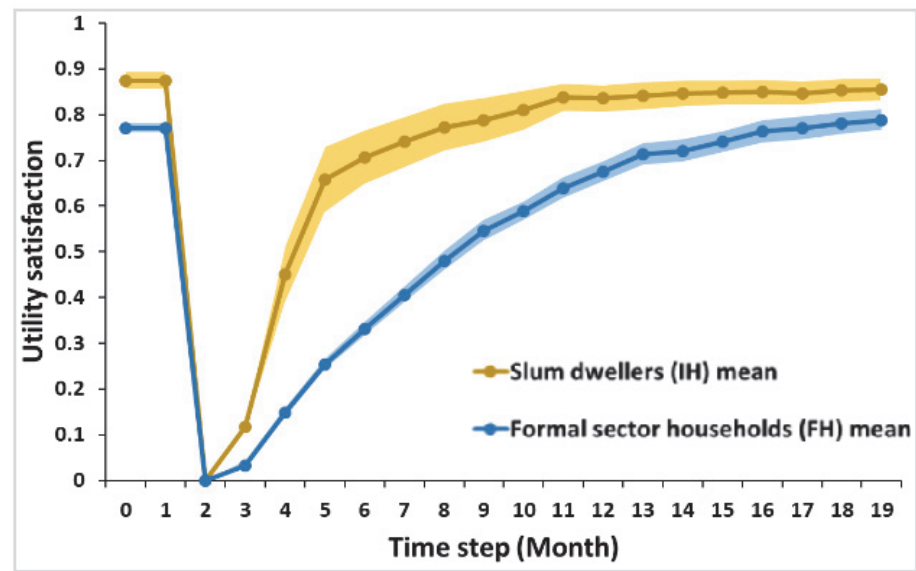

Figure 7.2 Mean standard deviation utility satisfaction of the families residing in formal (FH) and informal (IH) urban areas for different time steps in the model, in which each step is equal to a month and the time step $=1$ is the Haiyan disaster moment. 

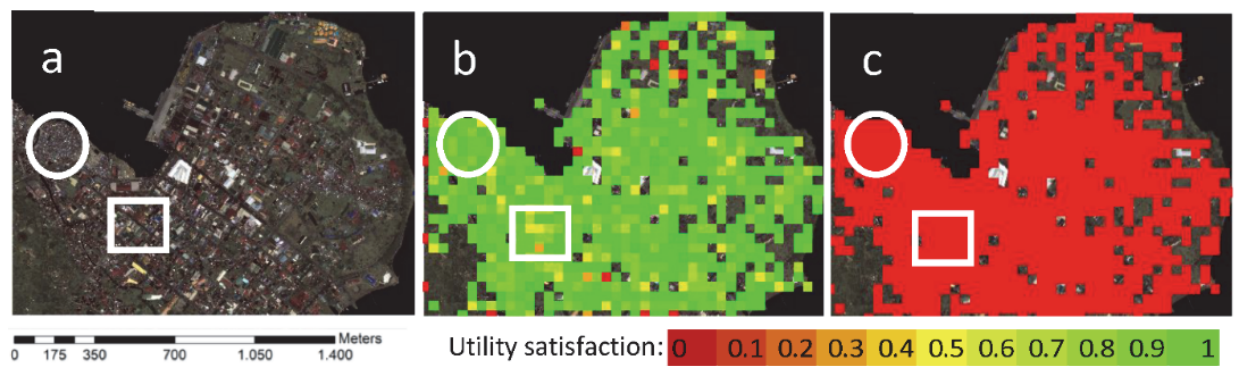

Utility satisfaction:
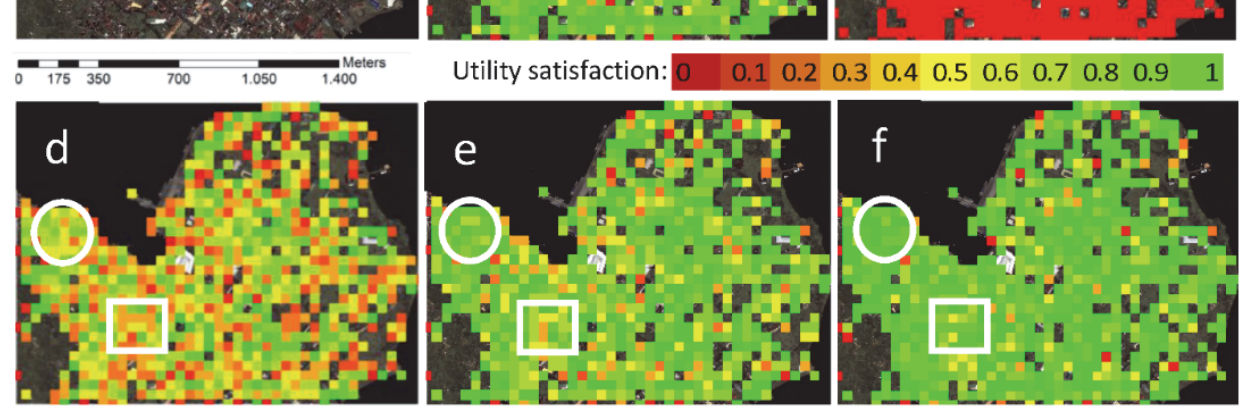

Figure 7.3 (a) Pre-Haiyan high-resolution satellite image of central Tacloban, (b-f) spatial distribution of the mean utility satisfaction for IHs and FHs for steps 0, 2, 5, 10, and 19 , respectively. The areas denoted with a circle and a rectangle denote informal and formal settlements, respectively. Each time step is equal to a month, and step $=1$ is the Haiyan disaster moment.

Figure 7.3 illustrates the spatial distribution of the mean utility satisfaction of the households for pre-disaster ( $($ step $=5), 8$ (step = 10), and 18 (step =19) months after the disaster. The areas denoted with a circle are informal settlements/slums, which recovered faster than the area denoted with a rectangle, which is formal settlements.

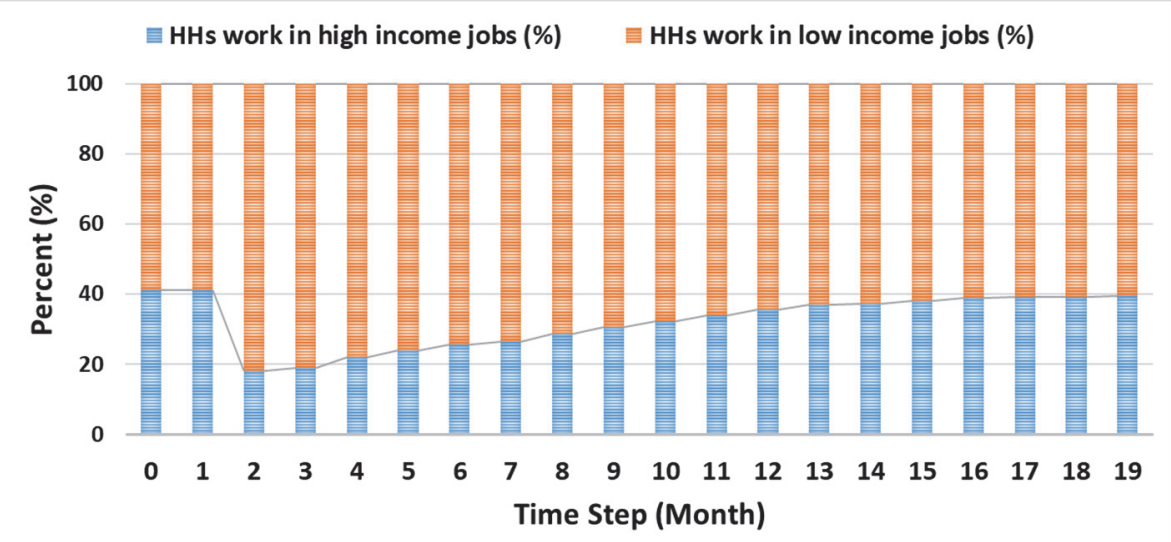

Figure 7.4 Percent of HHs work in high income versus low-income jobs in the postdisaster recovery process. Each time step is equal to a month, and step $=1$ is the disaster moment.

Figure 7.4 illustrates the dynamics in the percent of $\mathrm{HHs}$ working in high income and low-income jobs during the recovery. The results show an increase in $\mathrm{HHs}$ working in low-income jobs after the disaster ( $\mathrm{step}=1$ is the disaster 
moment) due to destruction of the high-income job places (e.g. factories) and $\mathrm{FH}$ (highly educated households) work in low-income jobs. However, by progressing with the reconstruction of the industrial buildings those $\mathrm{FH}$ have come back to high-income jobs to increase their utility satisfaction.

\subsubsection{Planned relocation and individual choices in the post- disaster recovery process}

One of the policies that have already been implemented in Tacloban is to move people away from the coastal strip, which is highly exposed to Typhoon-related hazards such as high winds and storm surges, to a relocation site in the designated safe zone North of Tacloban. Hence, we tested the scenario of having such a relocation site in the PDR model and to assess the effect of commuting distance in the post-disaster recovery process. We did this by adding new and available residential areas after the disaster only for slum households, the same as the actual policy in Tacloban. However, the distance to work (i.e. IH workplaces e.g. fishing) for IH who reside in this relocation site increases (i.e. 0.9 as the normalized distance to the workplace in the city), and has a significant impact on households' utility. Figure 7.5 shows the recovery curve (based on mean utility) of the $\mathrm{FH}$ and $\mathrm{IH}$ after the disaster. Accordingly, the results show that IHs recover faster in the early recovery phase by having an option of accommodation after the disaster; however, after two to three months the presence of relocation site has a low impact on the speed or quality of the recovery, but rather producing almost the same change in utility of the $\mathrm{IH}$. In addition, the recovery rates of the $\mathrm{IH}$ and $\mathrm{FH}$ are $102 \%$ and $109 \%$, where above $100 \%$ shows the better mean utility satisfaction than pre-disaster situation, and thus, reaching the build-back-better goal.

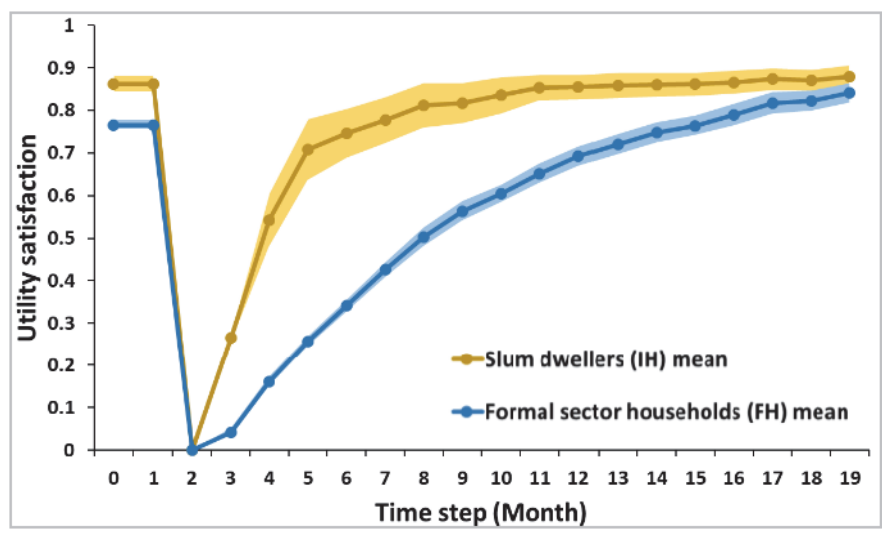

Figure 7.5 Mean and standard deviation utility satisfaction of the families residing in formal (FH) and informal (IH) urban areas for different time steps with the presence of relocation site. Each time step is equal to a month and the time step $=1$ is the disaster/Haiyan moment. 
The spatial distribution of the mean utility for $\mathrm{IH}$ and $\mathrm{FH}$ with relocation site availability after the disaster is generated and overlaid on the original image of the area for the pre-disaster and post-disaster periods (Figure 7.6). Moreover, the number of relocation site dwellers was computed for each of the selected steps and is illustrated for each step (Figure 7.6i). The results demonstrate the pattern of IH movement into and out of the relocation site in the post-disaster recovery period. Accordingly, IHs moved to the relocation site in the early recovery phase, where they do have at least houses to live in, and consequently, this increases their satisfaction. However, by progressing with the reconstruction process in the central part of the Tacloban, the occupation ratio of the relocation site is decreased (Figure 7.6). This shows that IHs prefer to move back to the same locations as the pre-disaster situation, which are closer to their workplaces, and this is an important factor in increasing their satisfaction. Accordingly, policy and decision-makers should consider the commute distance to workplaces as one of the influential factors in planning the recovery, in particular new settlement constructions, while trying to increase the resilience of the households by decreasing the hazard exposure and vulnerability. 

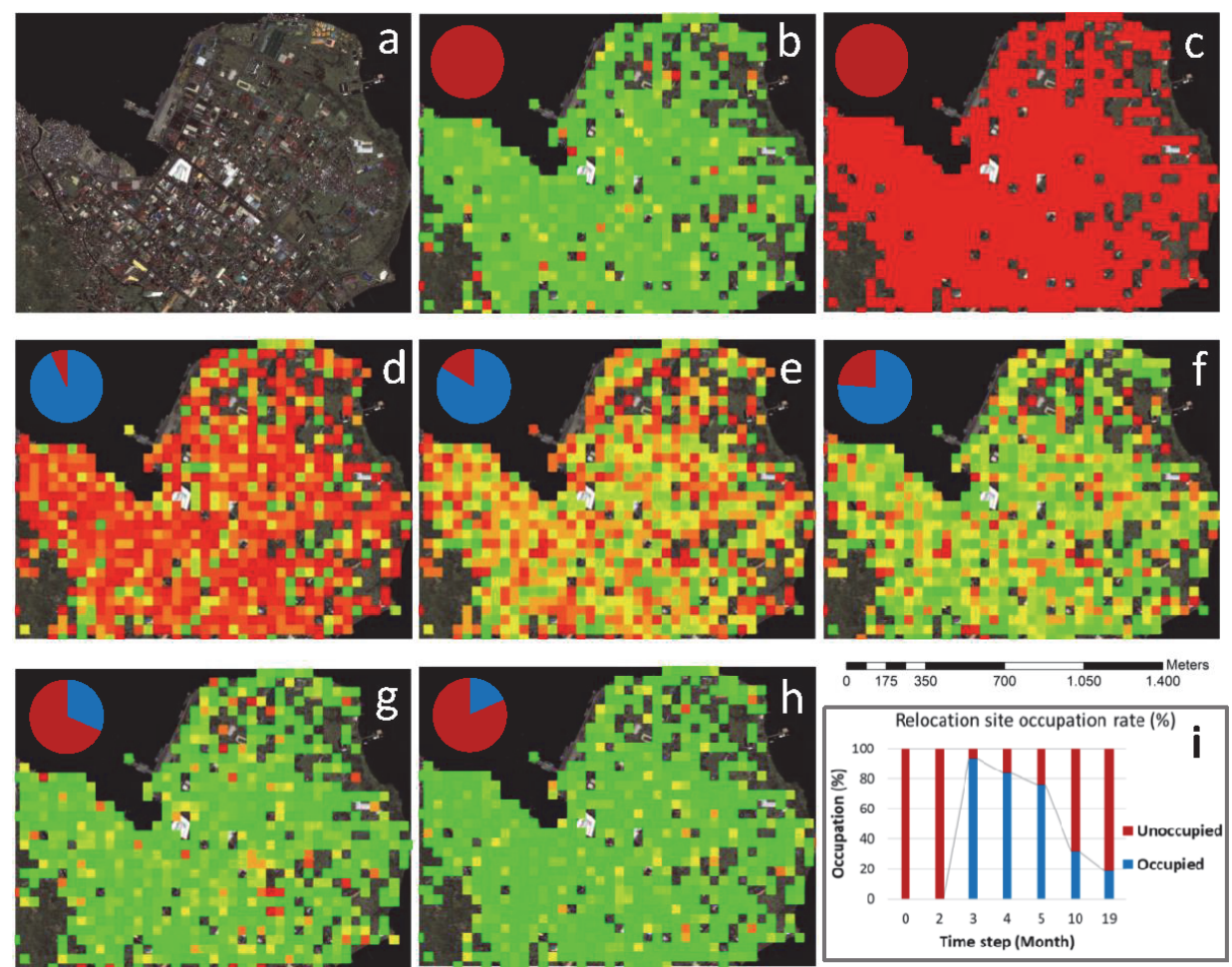

Utility satisfaction:

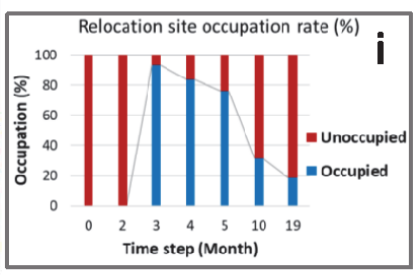

Figure 7.6 (a) Pre-Haiyan high-resolution satellite image of Tacloban urban area, (b-h) spatial distribution of the mean utility satisfaction for the His and FHs with the occupation ratio of the relocation site for steps $0,2,3,4,5,10$ and 19, respectively, and (i) is the relocation site occupied and unoccupied ratio for pre-and post-disaster situations. Each time step is equal to a month, and step $=1$ is the disaster moment.

\subsubsection{The effect of the employment rate on the post-disaster recovery process}

In the PDR model, the employment rate determines the probability of an agent having a job, which also has an impact on the calculation of agents' utility satisfaction (Appendix A. Eq.1). The utility satisfaction of an agent without a job will be zero, and it will look for new job opportunities or a new location and a job. This also becomes important in a post-disaster situation, in which there are job dynamics, and an agent may use its social contact network or individual processing to find a job based on its characteristics. According to the official statistics data (explained in section 2.1) for Tacloban, the employment rate in 2012 was 0.92 . However, the employment in the post-disaster situation is contingent on the financial aid received from national governments and international NGOs, expanding the range of feasible recovery pathways. Hence, we ran a sensitivity analysis changing the employment rate $[0.5 ; 0.7 ; 0.8]$ in addition to the actual rate (i.e. 0.92 ) to explore its effect on the post-disaster 
recovery process. As before, we report the dynamics of utility satisfaction unfolding during the recovery for IHs and FHs separately (Figure 7.7). The results demonstrate that the $\mathrm{FH}$ and $\mathrm{IH}$ recover (i.e. return to almost normal utility satisfaction) after the disaster at almost the same speed. Moreover, the IH recovery rates with the employment rates of $0.5,0.7,0.8$, and 0.92 are respectively $99 \%, 98 \%, 104 \%$, and $97 \%$, and the $\mathrm{FH}$ recovery rates with the employment rates of $0.5,0.7,0.8$, and 0.92 are respectively $104 \%, 103 \%$, $104 \%$, and $103 \%$. The above $100 \%$ recovery rates show the better mean utility satisfaction than pre-disaster one, and thus, reaching the build-back-better goal.

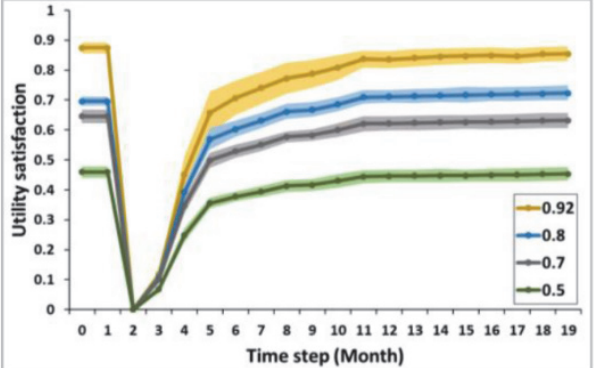

(a)

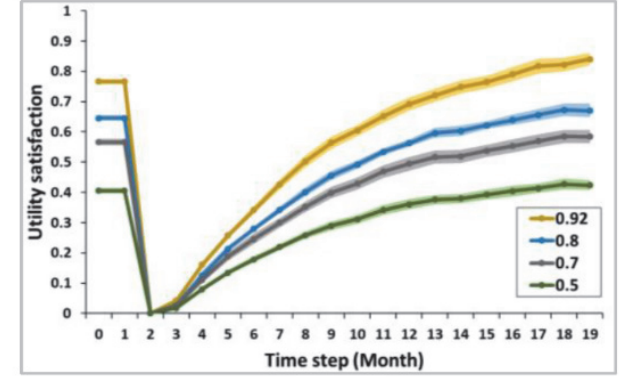

(b)

Figure 7.7 The mean and standard deviation utility satisfaction produced by the PDR model with different employment ratios $(0.5,0.7,0.8,0.92)$ for the IH (a) and FH (b) in different time steps. Each time step is equal to a month, and step $=1$ is the disaster moment.

\subsection{Conclusions}

In this paper, we develop a spatial ABM of post-disaster recovery to explore the behaviour of two distinct groups living in a city, i.e. families residing in the formal and informal urban areas. The urban area of the Tacloban city, the Philippines, which hit by Typhoon Haiyan on $8^{\text {th }}$ November in 2013 , is used as a case study to define the characteristics of the agents and create the environment. The objective of the study is to go beyond the physical assessment of the recovery process and investigate socio-economic reasons why some areas thrive while others languish in the post-disaster recovery process. We have integrated multi-temporal RS data and advanced machine learning methods to provide input to the ABM. To do so, we model the behavior of heterogeneous households who aim to satisfy their utility shaped by the individual socio-economical characteristics. We present the simulation results for two groups: slum dwellers (IH) and households residing in formal housing $(\mathrm{FH})$. Based on the insights gained from the PDR model we identify that IH recovers faster than $\mathrm{FH}$ and therefore, may appear more resilient. However, we question whether returning to the same state - which was already undesirable to begin with for $\mathrm{IH}$ - is ethically-acceptable in the presence of 
such inequalities, and runs counter to Sendai principles. Slum-dwellers suffer the most extensive damage during the disaster and develop no capacity to recover to a better state, even in the presence of new housing in a safe zone if it robs them of employment opportunities. It is also shown that this type of visualization provides insights into the neighbourhood level assessments, which can also be employed for other applications. To gain more insights and detailed information regarding the behaviour of the IH and FH groups, we define and test two policy scenarios: the construction of the relocation site and impact of the employment rate change on the recovery process and resilience of the households. We show that while the existence of a relocation site increased the IH recovery speed and thus their resilience, the employment rate has a small effect on the speed of the recovery for both IH and FH in terms of utility satisfaction measure. Furthermore, we demonstrate the importance of the commute distance to the workplace for $\mathrm{IH}$.

The results show that the basic aim of developed PDR model has been reached since it provides insight into different recovery patterns of the slum and formal sector households. Therefore, policymakers and governments can use the insights derived from the model to understand recovery rates at the neighbourhood level. However, detailed data are needed to replace the randomness/stochasticity in the model and understand the behavior of each individual and their dynamics during the recovery.

One of the limitations of this study is neglecting the effect of the natural hazard risk and risk perception of the individuals after such a major disaster. Hence, by adding the natural hazard risk components (e.g. exposure to hazard, vulnerability and prior disaster experience) to the PDR model, more accurate empirical implementation of the model can be obtained. Some of the information related to these components can also be extracted from RS data. Moreover, adding more variables/components to utility measure of the PDR model, such as social and economic characteristics of households (e.g., age and gender), can provide more insights into the recovery process.

\subsection{Appendix A. The ODD +D description of the $P D R$ agent-based model}

In this section, we describe the developed post-disaster recovery (PDR) model based on the ODD + D protocol (Müller et al. 2013). The ODD + D provides a standard description and documentation protocol for agent-based models, which guides researchers to sufficiently substantiate their model.

a. Overview

i. Purpose 
The developed post-disaster recovery (PDR) model simulates the decisionmaking process of individuals with respect to spatial mobility and job dynamic/selection based on the maximization of the satisfaction score computed according to socio-economic parameters for the households in urban areas of Tacloban, the Philippines. The PDR model provides a tool to understand how households (living in informal or formal settlements) select/change jobs and locations after a disaster during the reconstruction processes, based on the primary factors that influence their decisions after the disaster. In addition, the variable recovery across different neighbourhoods/sections of an affected urban area can be revealed by processing RS data; however, there is a need for effective tools to explain those observations and identify suitable means to influence the recovery process (find bottlenecks, etc.). The PDR model allows studying the different recovery rates of urban areas. Accordingly, policymakers and governments can use the insights derived from the model to understand why some regions may thrive and others languish (formal and informal settlements) and make effective decisions during the post-disaster recovery process.

\section{ii. Entities, state variables, and scales}

The main agents in the PDR model are households and buildings in an urban environment. Households could be of two types: families residing in formal (FH) or informal (IH) urban areas that have different behaviours, mostly due to their economic and education level. Further, households can be heterogeneous within each group, differentiated by income, education, workplace. Urban buildings could be of residential formal or informal and industrial buildings. The PDR model distinguishes between two types of residential areas - formal and informal (slums) as well as industrial buildings. The latter is important as they represent the location of mid- and high-income jobs, usually accessible only for $\mathrm{FH}$ with a higher level of education compared to IH. IH often have jobs in an informal sector, such as fishing or seasonal agricultural sector labour. The time interval of the model after the disaster is defined as a month.

\section{iii. Process overview and scheduling}

The overall process of the model is based on satisfying individual utility by choosing an action that gives them higher utility (Figure 7.8). This is derived from the agent's social and economic needs, which also defines the behaviour of the agent in the model. Let $\left(U\left(A_{i}\right)\right)(i=1,2,3, \ldots, N)$ represent the utility level of the agent, where $N$ is the number of agents, and $\left(A_{i}\right)$ is based on its current state in the model, then it will look for possible actions (options) ( $a_{i}$ ) to change its state and increase the current utility level. The possible actions for the agent $\left(A_{i}\right)$ are to move to a new location or changing its job, or both move to a new location and change its job. The agent can change its job or 
location using its social network or by looking for a list of available options individually. The lower utility level of an agent derived from its current state when compared to the others increases the chance of taking one of the possible actions in the model and changing its state. Accordingly, the information/state of the agent $\left(a_{i}\right)$ will be updated to its selected action from the previous step. Then, the utility $\left(U\left(a_{i}\right)\right)$ is calculated using the updated state of the agent based on the selected new action. The higher calculated utility from its current one will lead to taking action and change in the information of the agent for the next step.

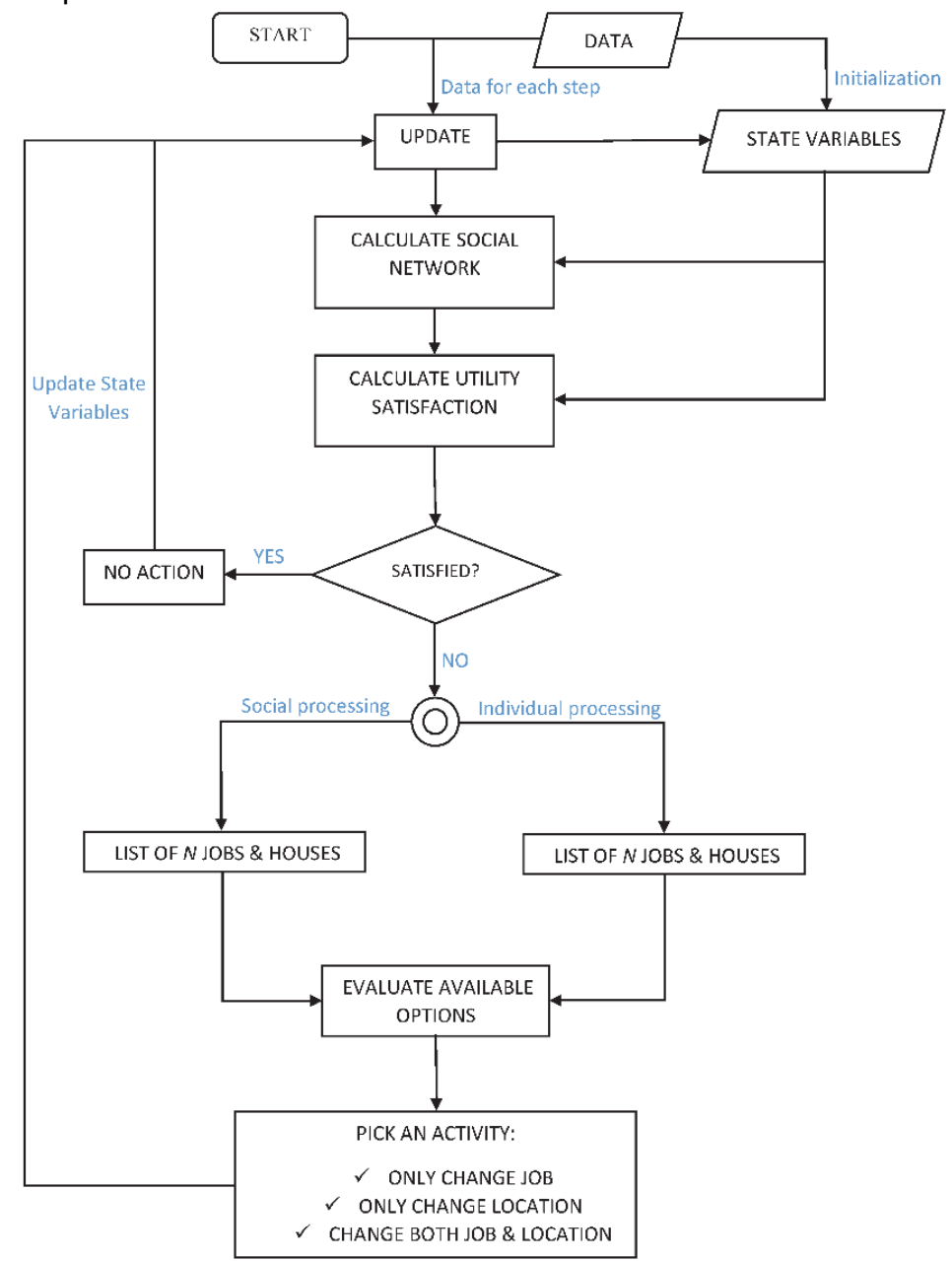

Figure 7.8 Conceptual flow framework of the PDR ABM.

b. Design concepts

i. Theoretical and empirical background

- Theoretical background: We mainly used two theories for developing the PDR model: the accessibility to the workplace (Alonso 1964), and the 
social capital (Lu 2017; Meikle et al.). The accessibility to the workplace is one of the influential factors in household choices (Alonso 1964), e.g., residential choice model (Roy and Lees 2020). According to the key interviews conducted during fieldwork in the area, it is also important after a disaster, while some buildings and workplaces can be destroyed, and some households may need to move or change jobs. We employed the distance to the workplace as a proxy for the accessibility analysis in the PDR model and used it to compute the satisfaction score $\left(U\left(A_{i}\right)\right)$ (see Eq. (1)).

The concept of social capital explains/defines the benefits of the contact networks between households and different social groups based on their social ties that can provide information regarding the opportunities in the communities (Meikle et al.). This means that households can have an impact on each other's decisions. In addition, social networks are one of the influential factors of individual activities in the community after a disaster and in the recovery phase by sharing the information in their social networks (Lu 2017). The similarity (homophily) and interactions between individuals are one of the important factors in making the connections and creating social contact networks (Mcpherson et al. 2001; Roy and Lees 2020). Hence, we employed the theory of homophily in the PDR model to define agent interactions. To do so, we first determined the contact network and then selected the information with the agent to select through the available opportunities provided by the networks.

- Empirical background: We used two information/data sources in the PDR model (explained in section 2.1): 1- Fieldwork data: key interviews, survey data (collected for the study (Kerle et al. 2019a)) and the demographic data collected from the Philippine Statistics Authority (e.g., unemployment rate, type of the economic activities before and after the disaster), 2- RS data, in particular, high-resolution satellite images acquired during pre- and post-disaster times. We extracted information from the machine learning-derived land cover, and land use maps using multi-temporal satellite images (the pre- and three days after disaster land use maps produced by (Sheykhmousa et al. 2019)) to be used in the PDR model.

ii. Individual decision making

The decision making of an agent $\left(A_{i}\right)$ relies on satisfying own utility $U\left(A_{i}\right)$ based on its current state with respect to living and working environment. Taking an action ( $\left.a_{i}\right)$ changes individual agent utility. The utility of an agent $\left(A_{i}\right)$ depends on its socio-economic and personal expectations defined by the agent's social level (i.e. education level). This score expresses how satisfied an agent is with living in a particular location, and having/not having a job, the balance between its income and education level, and which workplace it has to commute to each 
day for work. Accordingly, the utility for each agent can be computed using the following equation:

$U\left(A_{i}\right)=I * J *\left(\frac{Y}{E}\right) *(1-D) \quad$ (Eq. A. 1$)$

where $I$ represents the impact of the disaster, $J$ shows the job status, $Y$ and $E$ are the income and education levels, respectively, and $D$ is the normalized distance to the workplace for each agent $\left(A_{i}\right)$. Each variable of the utility can be determined as follow:

- I: shows if the agent has been impacted by the disaster and its house has been damaged or even destroyed. Thus, if the agent has a house damaged/destroyed $I=0$, and if not $I=1$. The damaged and intact buildings were extracted using RS-derived land use maps (explained in section 2.1).

- $\quad J$ : if the agent has a job $j=1$, and if not $j=0$. The job options for the highly educated households/agents (FH) are services (which are considered high-income jobs), farm, fishing, construction (which are in the group of low-income jobs). However, IH agents can only work in lowincome jobs. The selection of the unemployed agents is made based on the probability distribution, which is extracted from the data. Accordingly, the probability of having a job for each agent is 0.92 , while the probability of not having a job is 0.08 .

- $\quad Y$ : there are two income levels in the model: low and high. For an agent with high-income $Y=1$, and with low-income $Y=0.5$. Service sector employment falls in the high-income job group, while farm, fishing, and construction are in the low-income job category. Hence, FH agents prefer to work in high-income jobs, given that they are highly/better-educated agents, and IH can only work in low-income jobs. Assigning a workgroup to an agent is also based on the distributions extracted from data (Figure 7.9).

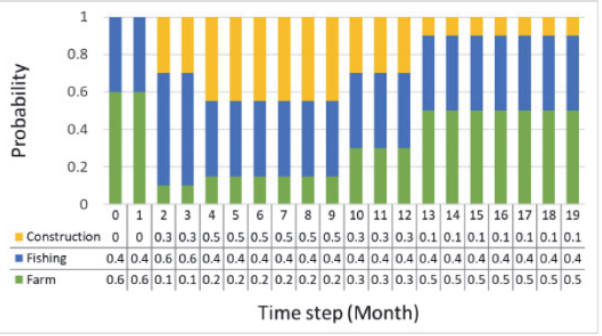

(a)

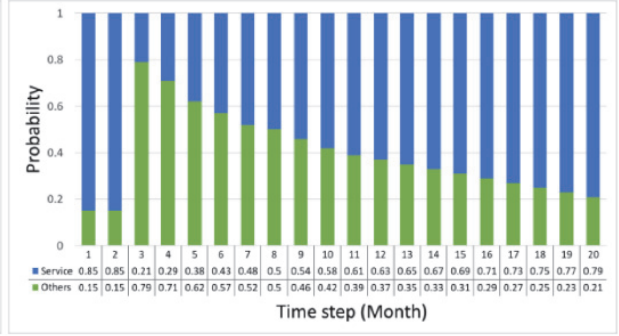

(b)

Figure 7.9 The probability distribution of the job sectors for IH (a) and FH (b) over time. Each time step is equal to a month, and step $=1$ is the disaster moment.

- $E$ : there are two education levels in the model. For an agent with high education level $E=1$, and with low education level $E=0.5$. 
Table 7.2 Explanation of the variables used for computing the utility satisfaction of the agents in each step.

\begin{tabular}{|c|c|c|c|}
\hline Variable & Description & Range of value & $\begin{array}{l}\text { Data used to } \\
\text { extract }\end{array}$ \\
\hline $\boldsymbol{I}$ & $\begin{array}{l}\text { Shows if the } \\
\text { agent has been } \\
\text { impacted by } \\
\text { the disaster }\end{array}$ & $\begin{array}{l}I=0 \text { if the agent's } \\
\text { house destroyed by the } \\
\text { disaster, and } I=1 \text { if the } \\
\text { agent's house remain } \\
\text { intact after the disaster }\end{array}$ & $\begin{array}{l}\text { Extracted from } \\
\text { remote sensing } \\
\text { data }\end{array}$ \\
\hline J & $\begin{array}{c}\text { Shows if the } \\
\text { agent has a job }\end{array}$ & $\begin{array}{c}J=0 \text { if the agent has } a \\
\text { job, and } J=1 \text { if not } \\
\text { has } a \text { job }\end{array}$ & $\begin{array}{l}\text { Initialized using } \\
\text { census data } \\
\text { (unemployment } \\
\text { rate) }\end{array}$ \\
\hline $\boldsymbol{Y}$ & $\begin{array}{c}\text { Income level of } \\
\text { the agent }\end{array}$ & $\begin{array}{c}Y=1 \text { for high-level } \\
\text { income, and } \\
Y=0.5 \text { for low level } \\
\text { income }\end{array}$ & $\begin{array}{l}\text { Extracted using } \\
\text { remote sensing } \\
\text { data }\end{array}$ \\
\hline $\boldsymbol{E}$ & $\begin{array}{c}\text { Education level } \\
\text { of the agent }\end{array}$ & $\begin{array}{c}E=1 \text { for high level } \\
\text { education, and } E=0.5 \\
\text { for low level education }\end{array}$ & $\begin{array}{l}\text { Extracted using } \\
\text { remote sensing } \\
\text { data }\end{array}$ \\
\hline$D$ & $\begin{array}{l}\text { Normalized } \\
\text { distance to the } \\
\text { workplace for } \\
\text { only high- } \\
\text { income jobs } \\
\text { (i.e. service) }\end{array}$ & $D \in[0.001,0.408]$ & $\begin{array}{l}\text { Extracted using } \\
\text { remote sensing } \\
\text { data }\end{array}$ \\
\hline
\end{tabular}

- $\quad D$ : is the distance to workplaces for the agents. The distance for FHs is based on the distribution that has been computed from the RS data (Figure 7.10). This distance was initially computed in a point-to-point manner using the pre-Haiyan land use map (using $\mathrm{FH}$ and industrial buildings locations/coordinates). Also, for IHs, it can be either 0.05 or 0.1 based on equal distribution. 


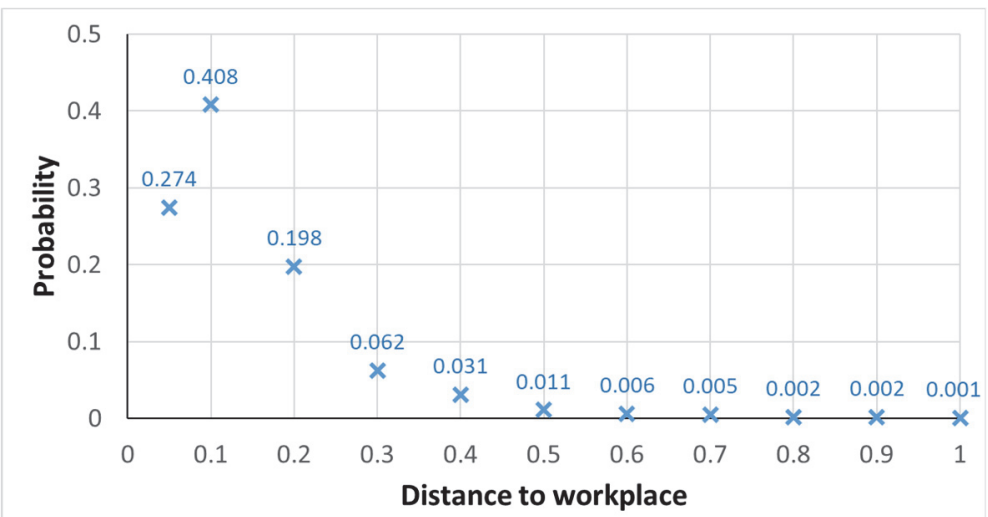

Figure 7.10 The probability distribution of the distance to the workplace (i.e. service job type) for FHs.

Based on the above-explained variables and their possible values, the utility of the agent will be $[0,1]$. For example, an agent without a house to live (when its house was destroyed by the disaster and has not yet been reconstructed in the recovery phase) or a job to have income, or has a workplace in the farthest place its $U\left(A_{i}\right)$ will be equal to zero.

Each step of the model is considered as one month, and during the recovery phase, the buildings might be reconstructed and can be added to the model, i.e. can be used for agents to live in. The monthly increase of the reconstructed buildings was extracted separately for formal buildings and slums using the machine learning-derive land use maps from satellite images (explained in section ).

iii. Individual sensing

The social contact network of each agent can be formed based on their social interactions in the social spaces like community centres and workplaces (Bandyopadhyay et al. 2011; Jackson et al. 2012; Oldenburg and Brissett 1982; Shughrue 2013; Wasserman and Faust 1994). Accordingly, we used the workplace of agents as a space to make the contact network in the model. First, we used the following equation to find the degree of the potential of being a contact network:

$C\left(A_{i}, A_{j}\right)=\operatorname{Sector}\left(A_{i}, A_{j}\right)+\gamma$

where $\operatorname{Sector}\left(A_{i}, A_{j}\right)$ is equal to one if the agents work in the same business sector, and if not it equals to zero. And, $\gamma \in[0,1]$ adds randomness to the interaction between agents.

Finally, the agents $A_{i}$ and $A_{j}$ may become a contact network and may have an impact on each other's decisions if $C\left(A_{i}, A_{j}\right)$ has a greater number than 0.8 . 


\section{iv. Interactions}

This section describes how the agents in their contact network (which are extracted using the previous section) make social networks and share information and opportunities in the model. To do so, we used the theory of homophily, in which the social network is based on the degree of similarity (homophily) between two agents. Accordingly, the agents with similar attributes have a higher degree of homophily and have more social impact on each other. This theory has been validated in several studies that use different attributes (e.g., age, education language) of the individuals in the social network (Chierchia and Coricelli 2015; Damon centola et al. 2005; Mcpherson et al. 2001). In addition, it has also been used for defining the social network for slum dwellers (Roy and Lees 2020).

In the PDR model, the degree of homophily is computed based on the similarity of the three agent attributes: job category/workplace, education, and income level. And it can be formulated as follows:

$\operatorname{Hom}\left(A_{i}, A_{j}\right)=\left|A t_{A_{i}} \cap A t_{A_{j}}\right| /\left|A t_{A_{i}}\right|$

where $\operatorname{Hom}\left(A_{i}, A_{j}\right) \in[0,1]$ is the degree of homophily and the greater number near to one the higher degree of homophily, $A t_{A_{i}}$ and $A t_{A_{j}}$ are the attributes of the $A_{i}$ and $A_{j}$, respectively. Hence, the weight of an edge between the agents $A_{i}$ and $A_{j}$ can be calculated using the equation below:

$\omega_{e}\left(A_{i}, A_{j}\right)=\operatorname{Hom}\left(A_{i}, A_{j}\right) * \frac{D_{A_{j}}}{\sum_{n} D_{A_{n}}} \quad$ (Eq. A.4)

where $D_{A_{j}}$ is the degree of agent $A_{j}$. Hence, based on the weight of the edges for each agent, the agents tend to have/select the same attributes as of the similar agents who have a high homophily degree.

After computing the homophily degree for the agent $A_{i}$ and ranking them, the first five agents were selected as the most influential ones. However, due to the monthly change of the status of the available job market in the recovery processes, the half weight of the influential agents is included in the model to have still the effect of the reconstruction process and job dynamics after the disaster.

\section{v. Individual prediction}

An individual/agent only in the case of finding a new opportunity that can increase its satisfaction score will change either its location or workplace or both at each step. However, deciding to look for new opportunities is based on the threshold value selection form the probability density distribution of the 
utility value in the model from the previous step. This means that if the number of agents with a specific utility value is larger than the others, the agents with that utility value will have a higher probability value to decide to seek new opportunities. Therefore, the threshold value as $T_{\text {change }}$ ranging from 0 to 1 for an agent $A_{i}$ to look for new opportunities for changing its current state can be computed using probability density function.

Then, if the utility value of the agent $A_{i}$ is below the $T_{\text {change }}$, the agent will decide to gather information and look for updating/changing its current state and increase its utility satisfaction. There are three options to change for each agent (only change job, only change location, change both job and location), of which the agent randomly selects one based on the equal probability for each. After selection of each of which, the agent starts gathering information for undertaking a new action using one of the following ways:

- Individual processing: randomly evaluating $N$ workplace or location or both from available options.

- Social networking: evaluating available options only for the workplace offered by its top $N$ social contact network.

Since the process of building reconstruction (including slums, formal and industrial buildings) changes the options available for the agents (workplace, household location) at each step, the reconstruction rate and job dynamics are the key components of the model. Thus, individual processing is based on the updated information on the recovery. However, the social networking process for evaluating options uses information from the previous step. Hence, the PDR model only uses the half weight of the output of the social network added to the half weight of the updates that come from the reconstruction processes. Eventually, after evaluating all the options, the agent decides to undertake an activity only if this will increase its current utility level.

\section{vi. Collectives}

Each agent (either $\mathrm{FH}$ or $\mathrm{IH}$ ) collects and stores information about his social contact network.

\section{vii. Stochasticity}

The process of selection of the social contact network is stochastic due to including the randomly selected number for parameter $\gamma$. In addition, the decision making of the agents/individuals has a randomness selection procedure first to choose whether to change workplace or location or both together and then a random selection from the probability distribution of the 
available options. This procedure adds stochasticity to the decision-making process of the agents in the PDR model.

\section{c. Details}

The initialization of the model and the employed input data are described in this section. The PDR model was developed and executed in Python using the MESA framework, and the residential choice model developed by (Roy and Lees 2020) was used as the base model. The source code is available on an openaccess library for $A B M$, which can be downloaded using the following link : (https://github.com/Saman-Gh933/Agent-based-modeling-of-post-disasterrecovery-with-remote-sensing-data).

\section{i. Initialization}

The PDR model initialized using the point-based land use data (FH, IH and location of the industrial workplaces). In total, the agents randomly assigned to $2131 \mathrm{IH}$ points and $1703 \mathrm{FH}$ points are initialized in the model. The agents' state variables are individual $\mathrm{IH}$ and $\mathrm{FH}$ households derived from the land use map of the Tacloban urban area, key interviews, census data, and survey data (from (Kerle et al. 2019a)). The PDR model started from one month before Haiyan to evolve. Hence, the PDR model started in September 2013 with a total number of 11,502 agents.

\section{ii. Input data}

There are two sources of the input data used in the model that change the conditions (available jobs, job types, and their probability distribution and open locations/houses) in each step. The damaged buildings and reconstruction levels of the built-up areas (slums and formal buildings) were extracted from the land use maps for three days after the disaster (for damage mapping and extracting the damaged houses), as well as three and eight months after the disaster. In addition, based on the best of our knowledge, we also assumed that the area had been fully reconstructed after five years. Subsequently, the extracted damage and reconstruction levels were transformed/translated to steps, given that each step of the model is one month. 


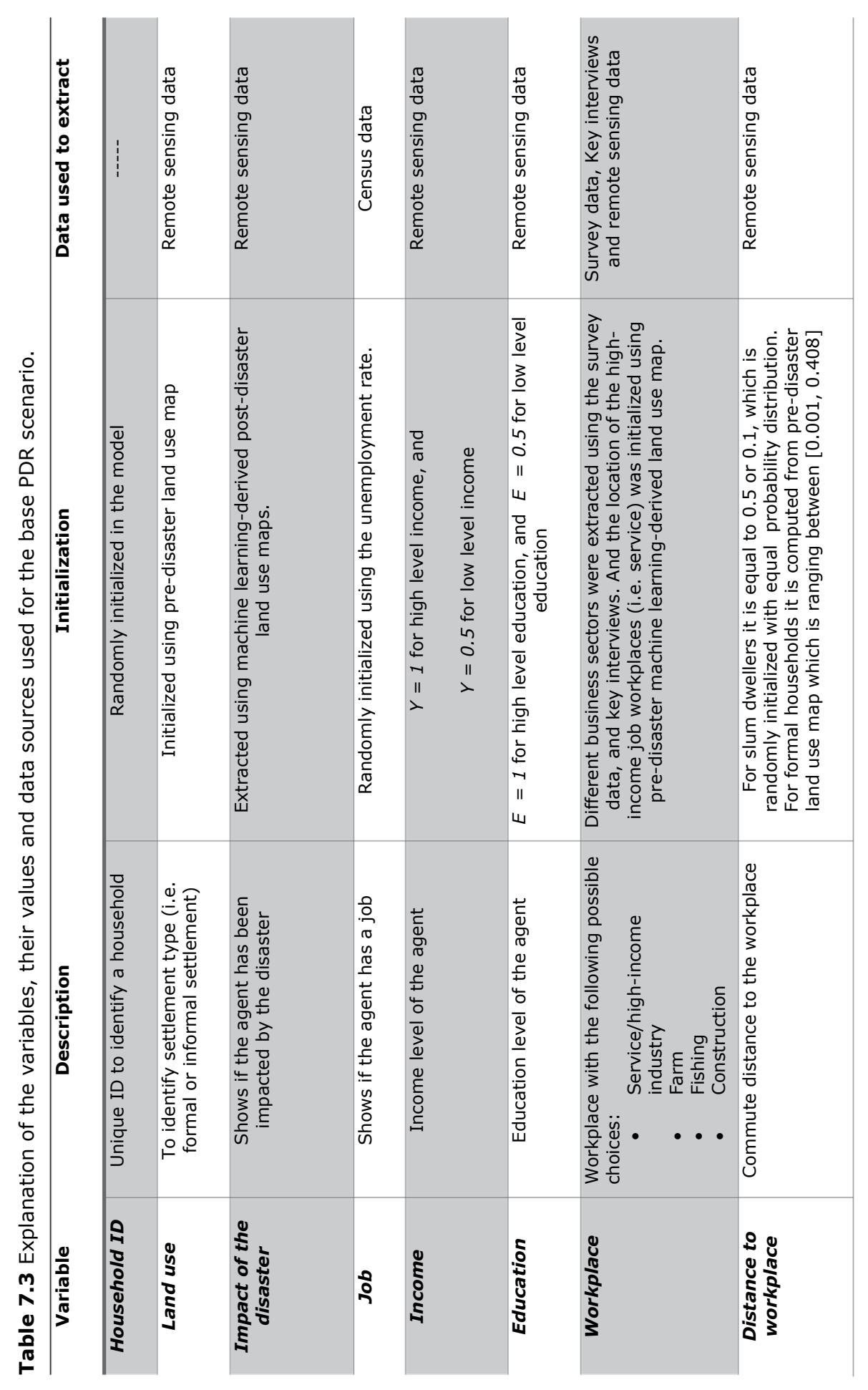




\subsection{References of Chapter 7}

Abebe, Y. A., Ghorbani, A., Nikolic, I., Vojinovic, Z., Sanchez, A., 2019. A coupled flood-agent-institution modelling (CLAIM) framework for urban flood risk management. Environmental Modelling \& Software, 111, 483492.

Alcayna T., Bollettino V., Dy P, Vinck P., 2016. Resilience and Disaster Trends in the Philippines: Opportunities for National and Local Capacity Building. PLOS Currents Disasters. doi: 10.1371/currents.dis.4a0bc960866e53bd6357ac135d740846.

Alonso, W., 1964. Location and landuse: Toward a general theorty of land rent. Harvard University Press.

An, L., 2012. Modeling human decisions in coupled human and natural systems: Review of agent-based models. Ecological Modelling, 229, 2536.

Bandyopadhyay, S., Rao, A., Sinha, B., 2011. Models for Social Networks With Statistical Applications. . Advanced Quantitative Technology in the Social Sciences: SAGE Publications.

Bank, T. W., 2018. DISASTER RISK MANAGEMENT IN THE CARIBBEAN: The World Bank's Approaches and Instruments for Recovery and Resilience. Retrieved from

Boston, M., Liu, Z., Jacques, C. C., Mitrani-Reiser, J., 2014. Towards assessing the resilience of a community in seismic events using agent based modeling. Paper presented at the Tenth U.S. National Conference on Earthquake Engineering, Anchorage, Alaska, U.S.

Brown, D., S., P., J., B., 2010. Disaster recovery indicators: Guidlines for monitoring and evaluation. University of Cambridge: Cambridge, UK: CURBE, Cambridge University for Risk in the Built Environment.

Brundiers, K., Eakin, H. C., 2018. Leveraging Post-Disaster Windows of Opportunities for Change towards Sustainability: A Framework. Sustainability, 10(5), 1390.

Burger, Oz, Kennedy, Crooks. 2019. Computational Social Science of Disasters: Opportunities and Challenges. Future Internet, 11(5), 103.

Chen, X., Zhan, F. B., 2008. Agent-based modeling and simulation of urban evacuation: Relative effectiveness of simultaneous and staged evacuation strategies. Journal of the Operational Research Society, 59(1), 25-33.

Chierchia, G., Coricelli, G., 2015. The impact of perceived similarity on tacit coordination: propensity for matching and aversion to decoupling choices. Frontiers in Behavioral Neuroscience, 9(202).

Ching Pk, De Los Reyes Vc, Sucaldito Mn, E., T., 2015. An assessment of disaster-related mortality post-Haiyan in Tacloban City. Western Pac Surveill Response J., 6(Suppl 1:34-8. pmid:26767133).

Coates, G., Li, C., Ahilan, S., Wright, N., Alharbi, M., 2019. Agent-based modeling and simulation to assess flood preparedness and recovery of manufacturing small and medium-sized enterprises. Engineering Applications of Artificial Intelligence, 78, 195-217.

CRED. 2015. The Human Cost of Natural Disasters.

CRED. 2018. Economic losses, poverty and disasters 1998-2017.

Damon centola, Robb willer, Michael macy. 2005. The Emperor's Dilemma: A Computational Model of Self-Enforcing Norms. American Journal of Sociology, 110(4), 1009-1040. 
Dawson, R. J., Peppe, R., Wang, M., 2011. An agent-based model for riskbased flood incident management. Natural Hazards, 59(1), 167-189.

Duarte, D., Nex, F., Kerle, N., Vosselman, G., 2018. Multi-resolution feature fusion for image classification of building damages with convolutional neural networks. Remote Sensing, 10(10), 1636.

Eid, M. S., El-Adaway, I. H., 2015. Optimizing Disaster Recovery Strategies Using Agent-Based Simulation Computing in Civil Engineering 2015 (pp. 379-386).

Eid, M. S., El-Adaway, I. H., 2017. Sustainable Disaster Recovery: MultiagentBased Model for Integrating Environmental Vulnerability into DecisionMaking Processes of the Associated Stakeholders. Journal of Urban Planning and Development, 143(1), 04016022.

Fan, C., Gong, L., Li, H., 2019. An agent-based model approach for assessing tourist recovery strategies after an earthquake: A case study of Jiuzhai Valley. Tourism Management, 75, 307-317.

Farmer, J. D., Foley, D., 2009. The economy needs agent-based modelling. Nature.

Fiedrich, F., Burghardt, P., 2007. Agent-based systems for disaster management. Commun. ACM, 50(3), 41-42.

Filatova, T., 2014. Market-based instruments for flood risk management: A review of theory, practice and perspectives for climate adaptation policy. Environmental Science \& Policy, 37, 227-242.

Filatova, T., Mulder, J. P. M., Van Der Veen, A., 2011. Coastal risk management: How to motivate individual economic decisions to lower flood risk? Ocean \& Coastal Management, 54(2), 164-172.

Ghaffarian, S., Kerle, N., 2019a. Post-disaster recovery assessment using multi-temporal satellite images with a deep learning approach. Paper presented at the 39th Earsel conference, Salzburg, Austria.

Ghaffarian, S., Kerle, N., 2019b. Towards post-disaster debris identification for precise damage and recovery assessments from UAV and satellite images. Int. Arch. Photogramm. Remote Sens. Spatial Inf. Sci., XLII-2/W13, 297302.

Ghaffarian, S., Kerle, N., Filatova, T., 2018. Remote Sensing-Based Proxies for Urban Disaster Risk Management and Resilience: A Review. Remote Sensing, 10(11), 1760.

Ghaffarian, S., Kerle, N., Pasolli, E., Jokar Arsanjani, J., 2019. Post-Disaster Building Database Updating Using Automated Deep Learning: An Integration of Pre-Disaster OpenStreetMap and Multi-Temporal Satellite Data. Remote Sensing, 11(20), 2427.

Grinberger, A. Y., Felsenstein, D., 2016. Dynamic agent based simulation of welfare effects of urban disasters. Computers, Environment and Urban Systems, 59, 129-141.

Jackson, M. O., Rodriguez-Barraquer, T., Tan, X., 2012. Social capital and social quilts: Network patterns of favor exchange. American Economic Review, 102(5), 1857-1897.

Kanno, T., Koike, S., Suzuki, T., Furuta, K., 2018. Human-centered modeling framework of multiple interdependency in urban systems for simulation of post-disaster recovery processes. Cognition, Technology \& Work, 21(2), 301-316. 
Kerle, N., Ghaffarian, S., Nawrotzki, R., Leppert, G., Lech, M., 2019a. Evaluating Resilience-Centered Development Interventions with Remote Sensing. Remote Sensing, 11(21), 2511.

Kerle, N., Nex, F., Gerke, M., Duarte, D., Vetrivel, A., 2019b. UAV-based structural damage mapping: A review. ISPRS International Journal of GeoInformation, 9(1), 14.

Lu, J., 2017. Chapter 10 - Interorganizational Network Dynamics in the Wenchuan Earthquake Recovery. In E. C. Jones \& A. J. Faas (Eds.), Social Network Analysis of Disaster Response, Recovery, and Adaptation (pp. 143-159): Butterworth-Heinemann.

Markhvida, M., Walsh, B., Hallegatte, S., Baker, J., 2020. Quantification of disaster impacts through household well-being losses. Nature Sustainability.

MCDEM. 2005. Focus on Recovery: A Holistic Framework for Recovery in New Zealand. MCDEM, New Zealand.

Mcnamara, D. E., Keeler, A., 2013. A coupled physical and economic model of the response of coastal real estate to climate risk. Nature Climate Change.

Mcpherson, M., Smith-Lovin, L., Cook, J. M., 2001. Birds of a Feather: Homophily in Social Networks. Annual Review of Sociology, 27(1), 415444.

Mehvar, S., Dastgheib, A., Filatova, T., Ranasinghe, R., 2019. A practical framework of quantifying climate change-driven environmental losses (QuantiCEL) in coastal areas in developing countries. Environmental Science \& Policy, 101, 302-310.

Meikle, S., Ramasut, T., Walker, J., Sustainable urban livelihoods: concepts and implications for policy.

Mishra, D., Kumar, S., Hassini, E., 2018. Current trends in disaster management simulation modelling research. Annals of Operations Research, 283(1-2), 1387-1411.

Mori, N., Kato, M., Kim, S., Mase, H., Shibutani, Y., Takemi, T., Tsuboki, K., Yasuda, T., 2014. Local amplification of storm surge by Super Typhoon Haiyan in Leyte Gulf. Geophysical Research Letters, 41(14), 5106-5113.

Müller, B., Bohn, F., Dreßler, G., Groeneveld, J., Klassert, C., Martin, R., Schlüter, M., Schulze, J., Weise, H., Schwarz, N., 2013. Describing human decisions in agent-based models - ODD $+D$, an extension of the ODD protocol. Environmental Modelling \& Software, 48, 37-48.

Nejat, A., Damnjanovic, I., 2012. Agent-based modeling of behavioral housing recovery following disasters. Computer-Aided Civil and Infrastructure Engineering, 27(10), 748-763.

Oldenburg, R., Brissett, D., 1982. The third place. Qualitative Sociology, 5(4), 265-284.

Roy, D., Lees, M., 2020. Understanding resilience in slums using an agentbased model. Computers, Environment and Urban Systems, 80, 101458.

Sheykhmousa, M., Kerle, N., Kuffer, M., Ghaffarian, S., 2019. Post-disaster recovery assessment with machine learning-derived land cover and land use information. Remote Sensing, 11(10), 1174.

Shughrue, C., 2013. A model of nonlinear urbanization and information flows across india. Hixon Center for Urban Ecology, 1(1), 1-19.

Sun, L., Stojadinovic, B., Sansavini, G., 2019. Agent-Based Recovery Model for Seismic Resilience Evaluation of Electrified Communities. Risk Analysis, 39(7), 1597-1614. 
Taylor, R. J. K., Forrester, J., Dreßler, G., Grimmond, S., 2015. Developing agent-based models for community resilience: connecting indicators and interventions.

Tesfatsion, L., 2005. Agent-based computationsl economics: A constructive approach to economic theory.

The World Bank, 2017. Results Brief - Climate Insurance. Retrieved from Available at https://www.worldbank.org/en/results/2017/12/01/climate-insurance:

UNISDR. 2015, 14-18 March. Sendai framework for disaster risk reduction 2015 - 2030. Paper presented at the Third World Conf. Disaster Risk Reduction, Sendai, Japan

Vetrivel, A., Gerke, M., Kerle, N., Vosselman, G., 2015. Identification of damage in buildings based on gaps in 3D point clouds from very high resolution oblique airborne images. ISPRS Journal of Photogrammetry and Remote Sensing, 105, 61-78.

Wang, H., Mostafizi, A., Cramer, L. A., Cox, D., Park, H., 2016. An agent-based model of a multimodal near-field tsunami evacuation: Decision-making and life safety. Transportation Research Part C: Emerging Technologies, 64, 86-100.

Wasserman, S., Faust, K., 1994. Social Network Analysis: Methods and Applications, Structural Analysis in the Social Sciences.: Cambridge University Press.

Watts, J., Morss, R. E., Barton, C. M., Demuth, J. L., 2019. Conceptualizing and implementing an agent-based model of information flow and decision making during hurricane threats. Environmental Modelling \& Software, 122, 104524.

Wooldridge, M., 2009. An introduction to multi agent systems. West Sussex: Wiley. 
Chapter 8 - Synthesis 


\subsection{Conclusions}

The main objective of this research was to analyze the suitability of the remote sensing and spatial economic modeling to support a comprehensive postdisaster recovery assessment by: reviewing and analyzing the remote sensingbased proxies for DRM and resilience, developing a conceptual framework for post-disaster recovery and resilience assessments, adopting machine learning approaches to improve the precision of the current remote sensing-based damage and recovery assessments, developing the deep learning-based methods for damage and recovery assessments and test it in actual case studies, analyzing the suitability of the cloud computing platforms for recovery monitoring, and analyzing and developing an agent-based model to explain the different post-disaster recovery patterns, and resilience. The main findings and conclusions of this study are sorted based on the initially designed research questions as follows:

\section{Research question 1: What are the state-of-the-art remote sensing- based proxies/indicators for disaster risk management and resilience assessment?}

To address this question, the available remote sensing-based proxies in urban Disaster Risk Management literature/studies were reviewed and analyzed, focusing on two risk elements typically associated with pre-disaster situations (i.e., vulnerability and resilience), and two post-disaster elements (i.e., damage and recovery). The identified proxies were grouped and reviewed in four primary environments and their corresponding sub-categories: built-up (buildings, transport, and others), economic (macro, regional and urban economics, and logistics), social (services and infrastructures, and socioeconomic status), and natural. The collected proxies can be used by researchers, governments, and disaster planners at any stage of the DRM cycle to assess targeted risk components, i.e., vulnerability, resilience, damage, and recovery. Furthermore, critical analysis and discussions were conducted to determine the strength and limitations of the current proxies in the field. In addition, insights from wider science fields such as social, economic, and urban form studies were provided to propose improvement directions for the proxies. The results indicated that there has been an increase in the number of proxybased studies in urban DRM. The results also imply the increase in studies that require indirect measurements such as socio-economic analysis. However, there are gaps in the field that demand further research. The current remote sensing-based proxies were mostly developed for the physical assessments in urban DRM. Yet, urban DRM assessments rarely go beyond aggregated proxies for the socio-economic environment, and the offered proxies are have very limited capacity to assess functional aspects. Disaster resilience is the least studied component of DRM in terms of developing remote sensing proxies. However, resilience has been shown as one of the significant components of 
DRM, which has been gaining increasing importance in the field. Analyzing the proxies also showed that in some cases the developed proxies can be interchangeably used with some modifications in other assessments. Only seven remote sensing-based proxies have been developed for resilience assessment, and only seven of the reviewed articles focused on resilience. These numbers indicate a significant need for further studies in resilience assessment.

\section{Research question 2: How to conceptualize post-disaster recovery assessment, including its different types and aspects based on remote sensing data?}

The remote sensing-based direct and indirect measurements were sorted to address the holistic post-disaster recovery assessment. The procedure includes physical and socio-economic assessments. Each of these measurements provides information regarding its corresponding time of acquired remote sensing image, for example, the number of buildings for pre-disaster or postdisaster time. However, recovery assessment is mainly based on two steps. First, to do damage assessment through comparing the just after the disaster with the pre-disaster situation and second, recovery assessment based on change detection over damaged elements at risks and post-disaster time to extract how much they have come back to the normal or pre-disaster situation and functioning. This procedure can be executed for any disaster at any time step, providing three-time step remote sensing data (e.g., high-resolution satellite images), i.e., pre, just after, and post-disaster times. In addition, evaluating and comparing the rate and speed of the recovery for the selected regions can provide insights for resilience assessment, assuming that the resilient areas recover faster after a disaster. The results of this study also revealed that the proposed conceptual framework provides a comprehensive basic framework for any general or detailed analysis for recovery assessment depending on the amount and type of the information provided for each step of evaluation.

Research question 3: How to automate the extraction of useful information from remote sensing data to evaluate the post-disaster damage and recovery processes?

The ultimate goal is to automate the entire post-disaster damage and recovery assessments, including physical and socio-economic aspects. However, the automatization should start at small steps by automatic extraction of the required information and status of the considered elements, and then aggregate them for comprehensive damage and recovery assessments. Hence, to address this question, one of the most critical elements for urban areas, i.e., buildings, was selected to automatically update databases at any time after a disaster for both damage and recovery assessments. the state-of-the-art deep learning methods was chosen to process the satellite images and perform the 
change detection and extraction works. A ResUnet-CRF model, which is based on Unet with residual connection followed by a Conditional Random Field (CRF) was developed to train and then update building databases. OpenStreetMap building maps after conducting a modification and novel automatic change detection process were employed as the automated training area selection approach. The results showed that the proposed approach produces robust results in updating the building database in different post-disaster scenarios, such as damaged, collapsed, reconstructed, newly built, and demolished buildings, using diverse building characteristics such as color, shape, and size of the buildings under challenging environmental conditions.

\section{Research question 4: How to increase the precision of remote sensing- based damage and recovery assessments?}

One of the primary limitations of the current damage and recovery assessments from remote sensing imagery is using structural rubble/debris as a proxy to detect damaged buildings/areas. However, disaster debris often includes vegetation, sediments, and relocated personal property in addition to structural rubble, i.e., items that are wind- or waterborne and not necessarily associated with the closest building. This leads to inaccuracies in buildingbased damage detection from remote sensing imagery since all assume the debris/rubbles belong to the closes building. In addition, traditionally, land cover classification-based damage detection has been categorizing debris as damaged areas. However, in particular in waterborne disasters such as tsunamis or storm surges, vast areas end up being debris-covered, effectively hindering actual building damage to be detected, and leading to an overestimation of the damaged area. Hence, the most emerging topic of increasing the accuracy of the remote sensing-based damage and, consequently, recovery assessments is to identify the debris types and find their origin. For building damage assessment, it is crucial to separate actual structural rubble from ephemeral debris. Accordingly, two approaches were investigated, first for debris identification from UAV images, and second from multi-temporal satellite images. To do so, three textural analysis approaches, i.e., Gabor filters, Local Binary Pattern (LBP), and Histogram of the Oriented Gradients (HOG), were implemented on mosaicked UAV images, and the relation between debris type and their time of removal was investigated using very high-resolution satellite images. The results showed that the HOG features, among other texture features, have the potential to be used for debris identification. In addition, multi-temporal satellite image analysis showed that debris removal time needs to be investigated using daily images because the removal time of debris may change based on the type of disaster and its location. 
Research question 5: To what extent cloud computing, i.e., Google Earth Engine, can be used to monitor the post-disaster recovery process?

Google Earth Engine (GEE) provides free access to vast amounts of remote sensing (RS) data and a powerful computing environment in a cloud platform, making it an attractive tool to analyze Earth's surface data. Hence, it was selected to be used as a cloud computing platform to monitor the recovery process after Typhoon Haiyan in a larger region, i.e., entire Leyte island that includes 62 municipalities in the Philippines, rather than only Tacloban city. Since Haiyan hit the island in November 2013, the best multi-temporal satellite images for this study, which are freely available in GEE, belong to Landsat 7 and 8 images. Hence, those images were utilized first to generate a cloud and shadow-free image composites and then perform a land cover classification for pre and post-disaster time steps. The experiments showed that GEE is a suitable platform for free and rapid cloud-based processes that also includes ready to use scripts and functions and useful guidance through the analysis. The results also revealed that the available Random Forest classification method is a reliable choice for a rapid large-scale land cover classification. In addition, to have an overview of the post-disaster recovery process for extensive regions, the Landsat 7 and 8 images are suitable, but higher spatial resolution images are needed for detailed analysis.

Research question 6: How to integrate multi-temporal remote sensing data with $A B M$ to assist in explaining the different recovery patterns? Remote sensing is an efficient tool to monitor and assess the recovery process after a disaster. However, it cannot explain the results since many other factors and actors cannot be tracked using remote sensing. To address this issue and develop a tool to explore different recovery patterns, an Agent-based modeling-based approach was developed mostly based on the remote sensing data. I integrated/used remote sensing with the ABM in two directions. First, the machine learning-derived land use maps extracted from remote sensing images were employed as the initial and step by step data sources, and second as a spatial domain to visualize and track the results. The model was used to understand and explore the post-disaster recovery patterns of the families residing in formal and informal (slum) urban areas, which follow different decision-making strategies in the recovery process. A utility satisfaction measure was proposed and generated for each group of agents. In addition, the disaster resilience of the targeted groups is assessed, assuming that the speed of the recovery is a proxy for resilience.

Furthermore, two realistic policy scenarios was simulated using the developed model: the construction of relocation sites after a disaster and the investments in improving employment options. The results were illustrated and the differences between informal and formal sector households recovery patterns 
in the base and selected scenarios were discussed based on their utility satisfactions. The results showed that the developed post-disaster recovery $A B M$ provides insight into different recovery patterns of the selected groups. Therefore, policymakers and governments can use the insights derived from the model to understand recovery rates at the neighborhood level. In addition, the results revealed that remote sensing in integration with machine learning can be used as a robust source of information to provide the required information for modeling.

\subsection{Reflections and outlook}

The aim of the proposed research was, on the one hand, the assessment of the post-disaster recovery with remote sensing, and the second was on explaining the different recovery patterns with spatial economic modeling. The first goal was addressed through a detailed investigation of the potential of the remote sensing for assessing the post-disaster recovery process from a holistic point of view, which includes functional assessments in addition to physical analysis. Remote sensing has been proved to be an effective and rapid tool to extract features and physical objects when processed with machine learning methods. In literature, there were quite a lot of papers at the time of conducting this research 2017 that address the feature and object extraction/detection using traditional machine learning methods or recent advanced ones, i.e., deep learning (Cheng and Han 2016). Those can also were employed to assess the status of the essential objects in post-disaster time, for example, detection of the damaged buildings and roads (Ural et al. 2011; Vetrivel et al. 2015; Vetrivel et al. 2016). However, socio-economic and functional assessments have to be elicited indirectly using proxies. Hence, the first goal was to develop new proxies for a comprehensive post-disaster recovery assessment. Shortly after starting to review the literature to extract the available remote sensing-based proxies, several issues raised up. For instance, the most important issue was to define proxy while nearly every type of passive RS being per se indirect, and therefore, every form of processing of such data inherently uses proxies. In addition, already several proxies were developed in different research fields without using any keyword for their indirect measurement or using different terminologies for such indirect interpretation/measurements, (e.g., indicator, proxy, index)(Ebert et al. 2009; Kotzee and Reyers 2016; Platt et al. 2016). Consequently, a comprehensive literature review was conducted at the start of the thesis in Chapter 2 to list the available remote sensing-based proxies for urban DRM and resilience, identify the limitations. and gaps, and provide insights from various perspectives for future studies. However, due to the mentioned problems in finding the relevant literature for the review, all the studies that used proxies were not included in the paper, while it has been tried to cover all of the developed proxies. In addition, the term "proxy" was defined and proposed as 
a keyword to address such measurements and make studies more organized then one can find the relevant studies more easier. Most of the relatively easy proxies were already developed by researchers in the DRM domain. For example, the presence of cracks and holes in facades of the buildings has been used as a proxy to assess damages to the buildings (Galarreta et al. 2015). However, there is a need for higher-level proxies that need indirect observability in addition to indirect interpretations to indicate something meaningful for computation. Higher-level proxies are crucial for addressing the functional assessment. Developing higher level remote sensing-based proxies is similar to solving a puzzle, trying to connect the different objects and features in data (e.g., extracted from remote sensing images) to interpret something meaningful. This makes developing higher-level proxies challenging. However, by increasing the spatial, spectral, and temporal resolutions of the remote sensing images as well as new tools to acquire data, the available objects and features for solving the puzzles will increase and lead to developing robust proxies. Already the results of this review were used by researchers to address the identified gaps (Castañeda-Garza et al. 2019; Hodicky et al. 2020) and develop new remote sensing-based proxies or combine them to address DRM (Derakhshan et al. 2020), and even using the term "proxy" as defined in this study in other domains rather than DRM (Lee and De Vries 2020). The proposed conceptual framework in Chapter 3 comprehensively addresses the aim of recovery assessment based on remote sensing data. It consists of two change detection approaches, in which the first one is for evaluating the damages, then using it as a benchmark for recovery assessment. The framework is a straightforward model that shows how to reach the goal of post-disaster evaluations at any time for any type of disaster, which is an extension of post-disaster damage assessment. Various types of remote sensing data can be used in the framework to have a comprehensive recovery assessment, integrating the physical assessment with the sorted proxies from Chapter 2 for socio-economic and functional assessments. Chapter 3 clearly shows the comprehensiveness and usefulness of the framework for assessing the damage and recovery processes. In addition, resilience can be evaluated, assuming that the recovery speed is a proxy for resilience. However, the results of Chapter 3 revealed that this assumption is not correct all the time. This might be due to the complexity of the assessments, and neglecting some other parameters that might lead to this result. Hence, there is a need to investigate and identify the relation between post-disaster recovery and its speed with resilience.

Chapters 2 and 3, in addition to conceptualization, identified the gaps in postdisaster recovery assessment with remote sensing. Accordingly, Chapters 4, 5 , and 6 addressed three of the current limitations and needs in recovery and resilience assessments. One of the identified limitations was in remote sensingbased damage assessment. Most of the remote sensing-based damage 
detection methods actually detect debris/rubble not damaged areas/buildings. Then assume that those detected debris are damaged areas or belong to the closest buildings, and thus, those buildings are damaged. However, this assumption is not entirely correct mostly in the case of water- and wind-borne disasters or in the case of multi-hazard event combined with an extensive storm surge, flooding or tsunami. Accordingly, there is a need to first identify the type of debris then one can associate them to region-based or structural damages. The proposed approaches in Chapter 4 for identifying the postdisaster debris for accurate damage and recovery assessments are fairly preliminary ones and need further investigations. However, it is the first study that brings this important point to attention of the researchers. Also using the feature extraction methods (e.g., HOG) showed promising results. Thus, as a future study, those features should be used in a machine learning classification procedure to extract the debris types. In addition, in this study it was not possible to use deep learning/CNN-based approaches due to lack of sufficient images and training samples; however, given the power such advanced methods, just using deep learning approaches (e.g., CNN) without preprocessing and feature extraction can produce robust results in identifying debris types. The idea of using the multi-temporal satellite images should be investigated further for a few days and weeks after a disaster to figure out its potential for debris identification. However, this highly depends on the characteristics of the region of interest (case studies), and in some cases, the type of disaster. So far, this study took the attention of the researchers and they started talking about this critical point (Baranwal et al. 2020; Nex 2019; Saffarzadeh et al. 2019); however, no method yet developed to directly address it.

The ultimate goal of fully automating the assessment was addressed in Chapter 5. To a large extent, this goal was reached with the proposed model. However, there is still a lot to do in this regard. At the start of this study and during the initial researches of this thesis, already the transition from traditional feature extraction-based machine learning (e.g., SVM, random forest )(Brunner et al. 2010; Gerke and Kerle 2011; Miura et al. 2013; Turker and Sumer 2008) to advanced deep learning/CNN models for building-based damage detection was completed and comprehensively studied. Accordingly, several advanced deep learning/CNN-based methods were already developed for post-disaster damage detection work (Ji et al. 2019; Vetrivel et al. 2017). However, all the studies required intensive manual work of extracting training samples for their developed CNN-based approaches. In addition, none of them went beyond the only damage assessment and monitor the changes in damaged buildings or other areas during the recovery process, which are addressed in this study. The idea of automating the training area selection procedure of an advanced deep learning method using OpenStreetMap produced reliable results for updating the building database. However, due to inaccuracies in the OSM 
building map required to develop a preliminary small modification step, which should be done manually. In addition, fine-tuning the change detection and deep learning parameters should be done by experts manually. Hence, developing a fully automated method not yet achieved. However, this was a great step forward in developing fully automated methods. Moreover, building database includes other aspects and details rather than the only location and damaged or not-damaged information. For example, the type and degree of the damages are of much importance. Although the proposed method produced robust results in detecting buildings and providing an overall overview of the building status after a disaster, yet it cannot be used for building footprint extraction. Accordingly, there is a need for exact building footprint extraction and updating the building databases with more details. The results of this study were already used in the literature, for example, to use OSM data for automatic training area selection for deep learning approaches (Ning et al. 2020). Furthermore, it is a pioneer study in automated post-disaster building map updating not only for damage time but also during recovery process (Nex et al. 2019; Shi et al. 2020; Sun et al. 2020).

In contrast to developed pixel-based deep learning methods in Chapter 5, which requires high computational power to process the data, Chapter 6 investigated the use of free cloud computing platforms, i.e., Google Earth Engine (GEE), for post-disaster recovery monitoring. GEE and cloud computing platforms were further improved their platforms and increased the available remote sensing data in their platforms and this attracts the researchers to do analysis in large/extensive regions (Ge et al. 2019; Mahdianpari et al. 2018; Sidhu et al. 2018; Stromann et al. 2019). This can also be employed for largescale post-disaster recovery monitoring, which was the aim of this study. The experiments showed that the GEE has a good potential to process the geospatial data, in particular freely available satellite images. However, the only available data in GEE for the considered case study were Landsat 7 and 8 satellite images. Despite the coarse resolution of the images, the results showed the potential of the images and the platform for rapid land cover classification of extensive regions. The coding environment of the GEE is userfriendly, but it uses JavaScript language, which is different from the popular programming languages for machine learning-based processes like Python. However, there are ready to use functions inside the platform, which makes it easier to implement the required methods. The available Random Forest classifier in the GEE also produced good accuracies in classifying different land covers from the Landsat images. However, the state-of-the-art deep learning methods are missing in the platform, which Google API is supposed to fill this gap and makes it possible to process the data in a Python programming language. Chapter 6 provided the first study on the use of cloud computing to monitor the post-disaster recovery process. However, it needs more future investigations in different directions, such as implementing new deep learning 
methods and adding very-high-resolution remote sensing data to assess the process in detail, in particular in complex urban environments.

The second underlying objective of this research was to develop spatial economic modeling to explain the different recovery patterns extracted with remote sensing. The goal was reached by developing a spatial economic model for post-disaster recovery in Chapter 7. As an effective spatial economic modelling, the agent-based modeling ( $A B M)$ was selected to be utilized in this study, which was already used and tested in different science fields and DRM domain as well (Filatova 2014; Filatova et al. 2011; Markhvida et al. 2020; Sun et al. 2019; Watts et al. 2019). The observations from the experiments conducted using $A B M$ for exploring and understanding the recovery process indicate that it is indeed a useful method to explain the different recovery patterns. In particular, the developed ABM was employed to explore the behavior of two distinct groups living in a city, formal and informal sector households. In addition, the proposed utility satisfaction measurement was a good choice to track the behavior change of the households. However, it needs more studies to add other parameters and components to have a complete utility satisfaction measure and increase the robustness of the model. The two scenario-based experimental studies also indicate the usefulness of the developed $A B M$ for forecasting and testing the different policy scenarios for the recovery process. There are many other scenarios and components that should be tested to carry out and understand their impacts on the recovery process. In addition, the observations indicate that the model can be used to assess the resilience, assuming that recovery speed is a proxy for resilience. However, the assumption itself needs more investigations to find the real relationship between recovery and resilience (Chapter 3 ).

In sum, the main contribution of this dissertation is three-fold. Firstly, it generates a catalog of remote sensing-based proxies for urban DRM and resilience, which can be used by researchers, governments, and decisionmakers. Secondly, it illustrates how remote sensing can be efficiently used for post-disaster damage, recovery, and resilience assessments through conceptualization, tackling the limitations of the current assessment methods using advanced machine learning techniques, development of automated deep learning-based approaches, and using cloud computing platforms. Thirdly, agent-based modeling sheds light on the reasons for different recovery patterns, and thus, resilience by exploring the critical socio-economic components and parameters of the recovery process. 


\subsection{References of Chapter 8}

Baranwal, E., Seth, P., Pande, H., Raghavendra, S., Kushwaha, S. K. P., 2020. Application of Unmanned Aerial Vehicle (UAV) for Damage Assessment of a Cultural Heritage Monument, Cham.

Brunner, D., Lemoine, G., Bruzzone, L., 2010. Earthquake Damage Assessment of Buildings Using VHR Optical and SAR Imagery. IEEE Transactions on Geoscience and Remote Sensing, 48(5), 2403-2420.

Castañeda-Garza, G., Valerio-Ureña, G., Izumi, T., 2019. Visual Narrative of the Loss of Energy after Natural Disasters. Climate, 7(10), 118.

Cheng, G., Han, J., 2016. A survey on object detection in optical remote sensing images. ISPRS Journal of Photogrammetry and Remote Sensing, $117,11-28$.

Derakhshan, S., Cutter, S. L., Wang, C., 2020. Remote Sensing Derived Indices for Tracking Urban Land Surface Change in Case of Earthquake Recovery. Remote Sensing, 12(5), 895.

Ebert, A., Kerle, N., Stein, A., 2009. Urban social vulnerability assessment with physical proxies and spatial metrics derived from air- and spaceborne imagery and GIS data. Natural Hazards, 48(2), 275-294.

Filatova, T., 2014. Market-based instruments for flood risk management: A review of theory, practice and perspectives for climate adaptation policy. Environmental Science \& Policy, 37, 227-242.

Filatova, T., Mulder, J. P. M., Van Der Veen, A., 2011. Coastal risk management: How to motivate individual economic decisions to lower flood risk? Ocean \& Coastal Management, 54(2), 164-172.

Galarreta, J. F., Kerle, N., Gerke, M., 2015. UAV-based urban structural damage assessment using object-based image analysis and semantic reasoning. Natural Hazards and Earth System Sciences, 15(6), 10871101.

Ge, Y., Hu, S., Ren, Z., Jia, Y., Wang, J., Liu, M., Zhang, D., Zhao, W., Luo, Y., Fu, Y., Bai, H., Chen, Y., 2019. Mapping annual land use changes in China's poverty-stricken areas from 2013 to 2018. Remote Sensing of Environment, 232, 111285.

Gerke, M., Kerle, N., 2011. Automatic Structural Seismic Damage Assessment with Airborne Oblique Pictometry $\subset$ Imagery. Photogrammetric Engineering \& Remote Sensing, 77(9), 885-898.

Hodicky, J., Özkan, G., Özdemir, H., Stodola, P., Drozd, J., Buck, W., 2020. Dynamic Modeling for Resilience Measurement: NATO Resilience Decision Support Model. Applied Sciences, 10(8), 2639.

Ji, M., Liu, L., Du, R., Buchroithner, M. F., 2019. A Comparative Study of Texture and Convolutional Neural Network Features for Detecting Collapsed Buildings After Earthquakes Using Pre- and Post-Event Satellite Imagery. Remote Sensing, 11(10), 1202. 
Kotzee, I., Reyers, B., 2016. Piloting a social-ecological index for measuring flood resilience: A composite index approach. Ecological Indicators, 60, 45-53.

Lee, C., De Vries, W. T., 2020. Bridging the Semantic Gap between Land Tenure and EO Data: Conceptual and Methodological Underpinnings for a Geospatially Informed Analysis. Remote Sensing, 12(2), 255.

Mahdianpari, M., Salehi, B., Mohammadimanesh, F., Homayouni, S., Gill, E., 2018. The First Wetland Inventory Map of Newfoundland at a Spatial Resolution of $10 \mathrm{~m}$ Using Sentinel-1 and Sentinel-2 Data on the Google Earth Engine Cloud Computing Platform. Remote Sensing, 11(1), 43.

Markhvida, M., Walsh, B., Hallegatte, S., Baker, J., 2020. Quantification of disaster impacts through household well-being losses. Nature Sustainability, 3, 538-547.

Miura, H., Midorikawa, S., Kerle, N., 2013. Detection of Building Damage Areas of the 2006 Central Java, Indonesia, Earthquake through Digital Analysis of Optical Satellite Images. Earthquake Spectra, 29(2), 453-473.

Nex, F., 2019. UAV-g 2019: Unmanned Aerial Vehicles in Geomatics. Drones, $3(3), 74$.

Nex, F., Duarte, D., Tonolo, F. G., Kerle, N., 2019. Structural Building Damage Detection with Deep Learning: Assessment of a State-of-the-Art CNN in Operational Conditions. Remote Sensing, 11(23), 2765.

Ning, H., Li, Z., Wang, C., Yang, L., 2020. Choosing an appropriate training set size when using existing data to train neural networks for land cover segmentation. Annals of GIS, 1-14.

Platt, S., Brown, D., Hughes, M., 2016. Measuring resilience and recovery. International Journal of Disaster Risk Reduction, 19, 447-460.

Saffarzadeh, A., Shimaoka, T., Nakayama, H., Afsari Fard, S., 2019. Lessons learned from the Ezgeleh-Sarpol Zahab earthquake of November 2017: status of damage and disposal of disaster waste. Waste Disposal \& Sustainable Energy, 1(4), 301-317.

Shi, W., Zhang, M., Zhang, R., Chen, S., Zhan, Z., 2020. Change Detection Based on Artificial Intelligence: State-of-the-Art and Challenges. Remote Sensing, 12(10), 1688.

Sidhu, N., Pebesma, E., Câmara, G., 2018. Using Google Earth Engine to detect land cover change: Singapore as a use case. European Journal of Remote Sensing, 51(1), 486-500.

Stromann, O., Nascetti, A., Yousif, O., Ban, Y., 2019. Dimensionality Reduction and Feature Selection for Object-Based Land Cover Classification based on Sentinel-1 and Sentinel-2 Time Series Using Google Earth Engine. Remote Sensing, 12(1), 76.

Sun, L., Stojadinovic, B., Sansavini, G., 2019. Agent-Based Recovery Model for Seismic Resilience Evaluation of Electrified Communities. Risk Anal., 39(7), 1597-1614. 
Sun, W., Bocchini, P., Davison, B. D., 2020. Applications of artificial intelligence for disaster management. Natural Hazards. https://doi.org/10.1007/s11069-020-04124-3.

Turker, M., Sumer, E., 2008. Building-based damage detection due to earthquake using the watershed segmentation of the post-event aerial images. International Journal of Remote Sensing, 29(11), 3073-3089.

Ural, S., Hussain, E., Kim, K., Fu, C.-S., Shan, J., 2011. Building Extraction and Rubble Mapping for City Port-au-Prince Post-2010 Earthquake with GeoEye-1 Imagery and Lidar Data. Photogrammetric Engineering \& Remote Sensing, 77(10), 1011-1023.

Vetrivel, A., Gerke, M., Kerle, N., Nex, F., Vosselman, G., 2017. Disaster damage detection through synergistic use of deep learning and 3D point cloud features derived from very high resolution oblique aerial images, and multiple-kernel-learning. ISPRS Journal of Photogrammetry and Remote Sensing.

Vetrivel, A., Gerke, M., Kerle, N., Vosselman, G., 2015. Identification of damage in buildings based on gaps in 3D point clouds from very high resolution oblique airborne images. ISPRS Journal of Photogrammetry and Remote Sensing, 105, 61-78.

Vetrivel, A., Kerle, N., Gerke, M., Nex, F., Vosselman, G., 2016. Towards automated satellite image segmentation and classification for assessing disaster damage using data specific features with incremental learning. Paper presented at the GEOBIA 2016, Enschede, The Netherlands.

Watts, J., Morss, R. E., Barton, C. M., Demuth, J. L., 2019. Conceptualizing and implementing an agent-based model of information flow and decision making during hurricane threats. Environmental Modelling \& Software, $122,104524$. 


\section{Summary}

One of the main components of the disaster risk management (DRM) cycle is recovery; however, it is the least understood one. Post-disaster recovery is the process of reconstructing communities in all their aspects (e.g., physical, economic, social, and environmental) in order to return life, livelihoods, and the built environment to their pre-impact or event better states. In this regard, timely and reliable information about the states of the damage and recovery process is vital for disaster planners and governments to make decisions. Remote sensing is an effective tool in providing information for post-disaster impact and recovery evaluations due to its agile data acquisition, synoptic perspective, growing range of data types, and instrument sophistication, as well as low cost. However, there is a need for diverse information to address the recovery process (including socio-economic aspects) comprehensively, and only a few of the parameters of interest can be extracted directly, while the majority have to be elicited indirectly.

In addition, although the variable recovery across different neighborhoods/sections of an affected area can be revealed by processing remote sensing data, there is a need for effective tools to explain those observations and identify suitable means to influence the recovery process (find bottlenecks, etc.). Spatial economic modeling, in particular, Agent-based modeling $(A B M)$, permits to explore the dynamics of the recovery process from the bottom up. In an $A B M$, agents (decision-making entities) interact with each other and their environments to decide and act based on defined rules for their behavior in a specific situation such as a recovery process. Therefore, it constitutes an opportunity for policy-makers to test different policy scenarios in an artificial simulation environment and explore their consequences. Accordingly, policy and decision-makers can take advantage of the simulation outcomes to steer the recovery process. According to the above-described two main issues, the objectives of this research were first to develop remote sensing-based approaches to assess the post-disaster recovery and second, to explain further and explore the recovery process using ABM. In this regard, several investigations were conducted, and several approaches were developed as summarized below:

1) Review of remote sensing-based proxies for disaster risk management: While several spatial and non-spatial parameters required for detecting and quantifying of DRM-related elements can be extracted directly from RS imagery, many have to be elicited indirectly. Hence, a comprehensive review of the current remote sensing-based proxies developed for urban DRM and resilience were conducted. The proxies were sorted for two risk elements typically associated with pre-disaster situations (vulnerability and resilience), and two post-disaster elements 
(damage and recovery). The proxies were reviewed in the context of four primary environments and their corresponding sub-categories: built-up (buildings, transport, and others), economic (macro, regional and urban economics, and logistics), social (services and infrastructures, and socioeconomic status), and natural. All environments and the corresponding proxies were discussed and analyzed in terms of their reliability and sufficiency in comprehensively addressing the selected DRM assessments. We highlight the strength and identify gaps and limitations in current proxies, including inconsistencies in terminology for indirect measurements. A systematic overview for each group of the reviewed proxies was presented that could simplify cross-fertilization across different DRM domains and may assist the further development of methods. While systemizing examples from the wider remote sensing domain and insights from social and economic sciences, a direction for developing new proxies was suggested, also potentially suitable for capturing functional recovery.

2) Post-disaster recovery assessment with remote sensing: A conceptual framework: The collected remote sensing-based information for pre- and post-disaster situations should have been used synergistically to address the post-disaster recovery assessment. Hence, a conceptual framework to monitor and evaluate the post-disaster recovery process and resilience was developed. In particular, available remote sensing imagebased proxies were used to assess the recovery addressing not-only physical but also functional aspects. Also, this conceptual framework can be used to evaluate disaster resilience, assuming that the speed of the recovery is a proxy for resilience assessment. The proxies were mostly extracted using machine learning-derived land cover and land use maps. The proposed approach was used to assess the recovery of barangays (municipalities), including Tacloban city, in the Leyte region in the central Philippines.

3) Improving the precision of the damage and recovery assessments through post-disaster debris identification: Most of the developed remote sensing-based damage detection methods have a common limitation, and it is using debris as a proxy for damage detection for both building/structural and regional damage detection. Thus, distinguishing the structural rubble from ephemeral debris can increase the accuracy of the damage and recovery assessments. The limitations of using debris and rubble piles as proxies for damage detection and subsequent post-disaster recovery assessment from remote sensing images were discussed, and two different approaches for post-disaster debris identification were investigated. Three feature extraction methods, i.e., Gabor filters, Local Binary Pattern (LBP), and Histogram of the Oriented Gradients (HOG), 
were investigated to identify the debris from UAV images. As the second strategy, an approach was proposed, which monitors the multi-temporal satellite images acquired days and weeks after the disaster to figure out the relation between debris type and their time of removal. The approaches were tested for Tacloban city using UAV and multi-temporal satellite images.

4) Automated deep learning-based post-disaster building database updating: The location of the damaged, reconstructed, and newly constructed buildings provide critical supporting information for both first responders and recovery planners after a disaster. The proposed method makes use of free OpenStreetMap building footprints available for a predisaster situation to automatically collect training areas from very-highresolution satellite images for a convolutional neural network (i.e., U-net) which is supported with residual connections. The trained network is then transferred and retrained for the post-disaster situation at any time after a simple building-based change detection analysis over OSM data. The proposed approach was tested for different scenarios of damage and recovery assessments in very high-resolution satellite images selected from Tacloban, the Philippines, after Typhoon Haiyan. The results showed that the proposed approach significantly decreased the manual work of training area collection, while maintaining the accuracy of the detected damaged, reconstructed, and newly constructed buildings at a high level.

5) Post-disaster recovery monitoring with Google Earth Engine: Most of the developed RS-based approaches in post-disaster damage and recovery assessments focus on the use of costly very-high-resolution data that require extensive digital storage and computing capacity to make use of them. However, cloud-based platforms such as Google Earth Engine (GEE) provide free RS data and computing power with a coding environment to develop and implement user-defined methods and process the data. Hence, the aim of this study was to test the suitability of GEE for a large-scale post-disaster recovery assessment. To do so, the GEE was employed to assess the recovery process over a three-year period after Typhoon Haiyan, which struck Leyte island, in the Philippines, in 2013. The following steps were developed and followed (i) generate cloud and shadow-free image composites from Landsat 7 and 8 satellite imagery and produce land cover classification data using the Random Forest method, and (ii) generate damage and recovery maps based on post-classification change analysis. The method produced land cover maps with accuracies $>88 \%$. The model was used to produce damage and three time-step recovery maps for 62 municipalities on Leyte island. The study showed that GEE has good potential for monitoring the recovery process for extensive regions. However, the most important limitation is the lack of very-high- 
resolution RS data that are critical to assess the process in detail, in particular in complex urban environments.

6) Agent-based modeling of the post-disaster recovery with remote sensing data: Recovery planners and decision-makers need to monitor and collect information about the ongoing post-disaster recovery process and understand the effect of different response strategies in dynamics. Monitoring tools/data such as geospatial platforms/remote sensing has been recently employed to assess the post-disaster recovery. However, remote sensing can mostly provide information regarding the physical aspects of the recovery, which are relatively easy to monitor and evaluate compared to functional assessments that include social and economic processes. Therefore, there is a need for a tool to understand and explore the impacts of different dimensions of the recovery, including the behavior of individual actors and their interactions with socio-economic institutions. An agent-based model was developed to simulate and explore the recovery process in urban areas of Tacloban, the Philippines devastated by Typhoon Haiyan in 2013. The recovery patterns of the formal and informal (slum) sector households, which follow different decision-making strategies in the recovery process, were studied differently. The satisfaction of the formal building and slum households were tracked and mapped to understand and demonstrate each of which recovery patterns. In addition, the disaster resilience of the targeted groups was assessed, given that the speed of the recovery is a proxy for resilience. Also, the effect of the unemployment rate and presence of a relocation site far from urban areas and workplaces after a disaster were experimented using the developed model. The developed post-disaster recovery model can be used by decision-makers to understand the recovery process and carry out the most influential factors and components. 


\section{Samenvatting}

Het minst begrepen onderdeel van de rampenrisicomanagementcyclus (RRM) is herstel na een ramp. Herstel na een ramp is het proces waarbij gemeenschappen in allerlei aspecten (e.g., fysiek, economisch, sociaal en milieu) worden gerestaureerd met als doel het leven en levensonderhoud te herstellen naar de staat van voor de ramp of zelfs naar een betere staat. In dit opzicht is tijdige en betrouwbare informatie over de toestand van het schadeen herstelproces van vitaal belang voor rampen managers en de regeringen om beslissingen te nemen. Remote sensing (RS)is een effectief hulpmiddel bij het verstrekken van informatie voor de evaluatie van de impact van een ramp en het herstel na een ramp, dankzij zijn wendbare data-acquisitie, zijn synoptische perspectief, het groeiende aantal RS datatypen en verfijnde RS instrumenten alsmede de lage kosten. $\mathrm{Er}$ is echter behoefte aan uiteenlopende informatie om het herstelproces (met inbegrip van de sociaaleconomische aspecten) uitgebreid aan te kaarten, en slechts enkele parameters kunnen direct worden geëxtraheerd, terwijl de meeste indirect ontlokt moeten worden. Bovendien, hoewel het variabele herstel in verschillende buurten/secties van een getroffen gebied kan worden geopenbaard door het verwerken van remote sensing gegevens, is er behoefte aan effectieve instrumenten om deze waarnemingen te verklaren en geschikte middelen te identificeren om het herstelproces te beïnvloeden (het vinden van knelpunten, enz.). Ruimtelijke economische modellering, met name Agent-based modeling (ABM), maakt het mogelijk om de dynamiek van het herstelproces van onderaf te onderzoeken. In $A B M$ werken agenten (besluitvormende entiteiten) met elkaar en met hun omgeving samen om te beslissen en te handelen op basis van gedefinieerde regels voor hun gedrag in een specifieke situatie, zoals een herstelproces. Dit biedt een kans voor beleidsmakers om verschillende beleidsscenario's in een kunstmatige simulatieomgeving te testen en de gevolgen ervan te onderzoeken. Beleids- en besluitvormers kunnen derhalve gebruik maken van de simulatieresultaten om het herstelproces te sturen. Op basis van de hierboven beschreven twee hoofdkwesties waren de doelstellingen van dit onderzoek ten eerste het ontwikkelen van op remote sensing gebaseerde methoden om het herstel na de ramp te beoordelen en ten tweede het nader uitleggen en onderzoeken van het herstelproces met behulp van ABM. In dit kader werden verschillende onderzoeken uitgevoerd en werden verschillende methoden ontwikkeld zoals hieronder samengevat:

1) Review van op remote sensing gebaseerde proxies voor rampen risicomanagement: Hoewel verschillende ruimtelijke en niet-ruimtelijke parameters die nodig zijn voor het detecteren en kwantificeren van DRMgerelateerde elementen direct uit RS-beelden kunnen worden geëxtraheerd, moeten veel van deze parameters indirect worden ontlokt. Daarom werd een alomvattende review uitgevoerd van de huidige op 
remote sensing gebaseerde proxies die zijn ontwikkeld voor stedelijke RRM en veerkracht. De proxies werden gesorteerd op twee risico-elementen die typisch verbonden zijn met situaties vóór de ramp (kwetsbaarheid en veerkracht), en twee elementen na de ramp (schade en herstel). De proxies werden beoordeeld in de context van vier hoofdomgevingen en hun corresponderende subcategorieën: bebouwd (gebouwen, vervoer en andere), economisch (macro-, regionale en stedelijke economie en logistiek), sociaal (diensten en infrastructuren en sociaaleconomische status) en natuurlijk. Alle omgevingen en de bijbehorende proxies werden bediscussieerd en geanalyseerd op het gebied van hun betrouwbaarheid en toereikendheid om de geselecteerde RRM-evaluaties uitgebreid aan te kunnen pakken. We belichten de kracht en identificeren tekortkomingen en beperkingen in de huidige proxies, inclusief inconsistenties in de terminologie voor indirecte metingen. Voor elke groep van de geëvalueerde proxies werd een systematisch overzicht gepresenteerd dat de kruisbestuiving tussen de verschillende RRM-domeinen kan vereenvoudigen en de verdere ontwikkeling van de methoden kan ondersteunen. Tijdens het systematiseren van voorbeelden uit het bredere remote sensing-domein en inzichten uit de sociale en economische wetenschappen werd een richting voorgesteld voor het ontwikkelen van nieuwe proxies, die mogelijk ook geschikt zijn voor het vastleggen van functioneel herstel.

2) Evaluatie van het herstel na de ramp met behulp van remote sensing: Een conceptueel kader: De verzamelde remote sensing informatie voor pre- en post-disaster-situaties had synergetisch moeten worden gebruikt om de evaluatie van het herstel na de ramp te kunnen adresseren. Daarom werd een conceptueel kader ontwikkeld om het herstelproces en de veerkracht na de ramp te monitoren en te evalueren. In het bijzonder werden de beschikbare remote sensing beeld-gebaseerde proxies gebruikt om het herstel te beoordelen, waarbij niet alleen fysieke maar ook functionele aspecten aan de orde kwamen. Ook kan dit conceptuele kader worden gebruikt om de veerkracht van rampen te evalueren, ervan uitgaande dat de snelheid van het herstel een proxy is voor de beoordeling van de veerkracht. De proxies werden meestal onttrokken met behulp van machine learning-verworven landbedekking en landgebruikskaarten. De voorgestelde werkwijze werd gebruikt om het herstel van barangays (gemeenten), waaronder Tacloban city, in de regio Leyte in het midden van de Filippijnen te beoordelen.

3) Verbetering van de nauwkeurigheid van schade- en herstelbeoordelingen door middel van puin-identificatie na de ramp: De meerderheid van de ontwikkelde remote sensing gebaseerde schade-detectiemethoden hebben een gemeenschappelijke beperking, het 
gebruik van puin als een proxy voor schade-detectie voor zowel bouwkundige/structurele als regionale schade-detectie. Door het onderscheid te maken tussen het structurele puin en het kortstondige puin kan de nauwkeurigheid van de schade- en herstelbeoordelingen dus worden verbeterd. De beperkingen van het gebruik van brokstukken en puinhopen als proxies voor de detectie van schade en de daaropvolgende beoordeling van het herstel na de ramp aan de hand van remote sensing beelden werden besproken, en er werden twee verschillende benaderingen voor de identificatie van brokstukken na de ramp onderzocht. Drie featureextractiemethoden, namelijk Gaborfilters, Local Binary Pattern (LBP) en Histogram of the Oriented Gradients (HOG), werden onderzocht om brokstukken uit UAV-beelden te identificeren. Als tweede strategie werd een aanpak voorgesteld, waarbij de multi-temporele satellietbeelden die dagen en weken na de ramp worden verkregen, worden gemonitord om de relatie tussen het type brokstukken en hun verwijderingstijdstip te achterhalen. De methodes werden getest op Tacloban stad met gebruik van UAV en multi-temporele satellietbeelden.

4) Geautomatiseerd en op deep-learning-gebaseerd updaten van een gebouwen-databank na een ramp: De locatie van de beschadigde, gereconstrueerde en nieuwgebouwde gebouwen biedt cruciale ondersteunende informatie aan zowel de eerstehulpverleners als de herstelplanners na een ramp. De hier voorgestelde methode maakt gebruik van kosteloze OpenStreetMap gebouw footprints die beschikbaar zijn voor een situatie vóór de ramp om automatisch trainingsgebieden te verzamelen van zeer hoge resolutie satellietbeelden voor een convolutioneel neuraal netwerk (d.w.z. U-net) dat wordt ondersteund met residuele verbindingen. Het getrainde netwerk wordt vervolgens op elk gewenst moment overgedragen en opnieuw getraind voor de situatie na de ramp, na een eenvoudige veranderingsdetectie analyse op basis van de OSM gegevens. De voorgestelde aanpak werd getest voor verschillende scenario's van schade en herstel evaluaties in zeer hoge resolutie satellietbeelden geselecteerd uit Tacloban, de Filippijnen, na Typhoon Haiyan. De resultaten toonden aan dat de voorgestelde methode de handmatige werkzaamheden voor het verzamelen van trainingsgebieden aanzienlijk heeft verminderd, terwijl de nauwkeurigheid van de gedetecteerde beschadigde, gereconstrueerde en nieuwgebouwde gebouwen op een hoog niveau is gebleven.

5) Het monitoren van het herstel na de ramp met Google Earth Engine: De meeste van de ontwikkelde op RS gebaseerde methodieken voor schade- en herstelbeoordelingen na een ramp zijn gericht op het gebruik van kostbare gegevens met een zeer hoge resolutie die een uitgebreide digitale opslag- en rekencapaciteit vereisen om er gebruik van te kunnen 
maken. Cloud-gebaseerde platformen zoals Google Earth Engine (GEE) bieden echter kosteloze RS-data en rekenkracht aan binnen een codeeromgeving om door de gebruiker gedefinieerde methoden te ontwikkelen en te implementeren en de data te verwerken. Het doel van deze studie was dan ook om de geschiktheid van GEE voor een grootschalige beoordeling van het herstel na een ramp te testen. Om dit mogelijk te maken werd de GEE ingezet om het herstelproces te beoordelen over een periode van drie jaar na Typhoon Haiyan, dat in 2013 op het eiland Leyte, in de Filippijnen, toesloeg. De volgende stappen zijn ontwikkeld en uitgevoerd (i) het genereren van wolken- en schaduwvrije beeldcomposieten uit Landsat 7 en 8 -satellietbeelden en het produceren van landbedekkingsclassificatiegegevens met behulp van de Random Forest-methode, en (ii) het genereren van schade- en herstelkaarten op basis van een analyse van de veranderingen post-classificatie. De methode leverde landkaarten op met een nauwkeurigheid van $>88 \%$. Het model werd gebruikt voor het maken van schade- en drie temporeel opvolgende herstelkaarten voor 62 gemeenten op het eiland Leyte. Uit het onderzoek is gebleken dat GEE een goed potentieel heeft om het herstelproces voor omvangrijke regio's te monitoren. De belangrijkste beperking is echter het ontbreken van RS-data met een zeer hoge resolutie die van cruciaal belang zijn om het proces in detail te kunnen beoordelen, met name in complexe stedelijke omgevingen.

6) Agent-based modellering van het herstel na de ramp met remote sensing data: Herstelplanners en beleidsmakers moeten toezicht houden op en informatie verzamelen over het lopende herstelproces na de ramp en het effect van verschillende responsstrategieën op de dynamiek begrijpen. Monitoringsinstrumenten/gegevens zoals geospatiale platforms/remote sensing zijn recentelijk gebruikt om het herstel na de ramp te beoordelen. Remote sensing kan echter vooral informatie opleveren over de fysieke aspecten van het herstel, die relatief eenvoudig te controleren en te evalueren zijn in vergelijking met functionele beoordelingen die sociale en economische processen omvatten. Daarom is er behoefte aan een hulpmiddel om de impact van de verschillende dimensies van het herstel te begrijpen en te verkennen, inclusief het gedrag van de individuele actoren en hun interacties met de sociaaleconomische instellingen. Een agent-based model werd ontwikkeld om het herstelproces in stedelijke gebieden van Tacloban, de Filippijnen verwoest door Typhoon Haiyan in 2013 te simuleren en te verkennen. De herstelpatronen van huishoudens in de formele en informele (sloppenwijk) sector, die verschillende besluitvormingsstrategieën in het herstelproces volgen, werden verschillend bestudeerd. De tevredenheid van de formele gebouwen- en krottenwijkhuishoudens werd gevolgd en in kaart gebracht om de herstelpatronen te begrijpen en te demonstreren. Daarnaast is de 
rampbestendigheid van de doelgroepen beoordeeld, aangezien de snelheid van het herstel een indicatie is voor de veerkracht. Met behulp van het ontwikkelde model werd ook geëxperimenteerd met het effect van het werkloosheidspercentage en de aanwezigheid van een verplaatsingslocatie ver van stedelijke gebieden en werklocaties na een ramp. Het ontwikkelde model voor herstel na een ramp kan door besluitvormers worden gebruikt om het herstelproces te begrijpen en de meest invloedrijke factoren en componenten uit te voeren. 


\section{Biography}

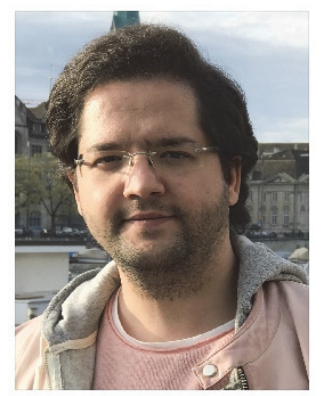

Saman Ghaffarian was born on 5th June 1985 in Tabriz, Iran. He received a $\mathrm{BSc}$ degree in Civil Engineering at the Azad University of Tabriz, Iran, in 2008. He worked for the industry as a civil engineer for four years. In 2012, he started an MSc program in Geomatics Engineering at Hacettepe University, Ankara, Turkey, and he received his MSc degree in 2015. He then joined the Intelligent Transportation Systems (ITS) laboratory at Boğaziçi University, Istanbul, Turkey as a guest researcher and worked on the use of remote sensing and machine learning for traffic and transportation engineering. In 2016, he started as a Ph.D. candidate at the Faculty of Geo-Information Science and Earth Observation (ITC), University of Twente, the Netherlands - which has resulted in the present manuscript. During his Ph.D. studies, he achieved two project application-based grants from Digital Global Foundation and the European Space Agency, providing free very-high-resolution satellite imagery for his Ph.D. project worth ca. 150 thousand euros. He has published various papers in leading remote sensing and computer science journals and his review paper (Chapter 2 of this manuscript) was featured on United Nations - SPIDER, PreventionWeb, and reliefweb websites. He also won the Remote Sensing journal 2019 travel award.

\section{Author's publications}

\section{Peer-reviewed journal articles}

1. Ghaffarian, S., Roy, D., Filatova, T., Kerle, N., 2020. Agent-based modelling of postdisaster recovery with remote sensing data. International Journal of Disaster Risk Reduction (Under review).

2. Ghaffarian, S., Rezaie Farhadabad, A., Kerle, N., 2020. Post-disaster recovery monitoring with Google Earth Engine. Appl. Sci., 10, 4574

3. Kerle, N.; Ghaffarian, S., Nawrotzki, R.; Leppert, G.; Lech, M., 2019. Evaluating resilience-centered development interventions with remote sensing, Remote Sensing, 11, 2511.

4. Ghaffarian, S., Kerle, N.; Pasolli, E.; Jokar Arsanjani, J., 2019. Post-disaster building database updating using automated deep learning: An integration of pre-disaster OpenStreetMap and multi-temporal satellite data. Remote Sensing, 11, 2427.

5. Sheykhmousa, M.; Kerle, N.; Kuffer, M.; Ghaffarian, S., 2019. Post-Disaster Recovery Assessment with Machine Learning-Derived Land Cover and Land Use Information. Remote Sensing, 11, no. 10, 1174. 
6. Ghaffarian, S., Turker, M., 2019. An improved cluster-based snake model for automatic agricultural field boundary extraction from high spatial resolution imagery. International Journal of Remote Sensing, 1-31.

7. Ghaffarian, S., Kerle, N.; Filatova, T., 2018. Remote sensing-based proxies for urban disaster risk management and resilience: A review. Remote Sensing, 10, 1760.

8. El merabet, Y., Ruichek, Y., Ghaffarian, S., Samir, Z., Bouijiha, T., Touahni, R., Messoussi, R., Sbihi, A., 2017 Maximal similarity based region classification method through local image region descriptors and Bhattacharyya coefficient-based distance: Application to horizon line detection using wide-angle camera. Neurocomputing, 265, 28-41.

9. Ghaffarian, S., Gokasar, I., 2016. Automatic vehicle detection based on automatic histogram-based fuzzy c- means algorithm and perceptual grouping using very highresolution aerial imagery and road vector data. Journal of Applied Remote Sensing, 10.

10. Ghaffarian, S., Ghaffarian, S., 2014. Automatic histogram-based fuzzy c-means clustering for remote sensing imagery. ISPRS Journal of Photogrammetry and Remote Sensing, 97, 46-57.

11. Ghaffarian, S., Ghaffarian, S., 2014. Automatic building detection based on purposive fastica (PFICA) algorithm using monocular high resolution Google Earth images. ISPRS Journal of Photogrammetry and Remote Sensing, 97, 152-159.

12. Ghaffarian, S., 2014. A kernel-based post-process for image segmentation using GVF snake. Journal of Automation and Control Engineering, 2, 3, 227-281.

\section{Book chapters}

13. El merabet, Y., Ruichek, Y., Ghaffarian, S., Samir, Z., Bouijiha, T., Touahni, R., Messoussi, R., 2016. Horizon line detection from fisheye images using color local image region descriptors and Bhattacharyya coefficient-based distance. In International Conference on Advanced Concepts for Intelligent Vision Systems, Springer, Cham, pp 58-70.

\section{Peer-reviewed conference papers and presentations}

14. Ghaffarian, S., Kerle, N., 2019. Post-disaster recovery assessment using multitemporal satellite images with a deep learning approach. Presented at 39th EARSeL conference 2019, Salzburg, Austria. (Remote Sensing journal travel award 2019 winner).

15. Ghaffarian, S., Kerle, N., 2019. Towards post-disaster debris identification for precise damage and recovery assessments from UAV and satellite images. Int. Arch. Photogramm. Remote Sens. Spatial Inf. Sci. XLII-2/W13: 297-302.

16. Ghaffarian, S., Kerle, N.; Filatova, T., 2018. A review of remote sensing-based proxies and data processing methods for urban disaster risk management. EGU General Assembly, Vienna, Austria 2018.

17. El merabet, Y., Ruichek, Y., Ghaffarian, S., Samir, Z., Bouijiha, T., Touahni, R., Messoussi, R., Sbihi, A., 2107. Hellinger kernel-based distance and local image region descriptors for sky region detection from fisheye images. In VISIGRAPP, pp 419-427. 
18. Ghaffarian, S., Ghaffarian, S., El merabet, Y., Samir, Z., Ruichek, Y., 2016. Automatic building roof segmentation based on PFICA algorithm and morphological filtering from LiDAR point clouds. In 37th Asian Conference on Remote Sensing (ACRS 2016), Colombo, Sri Lanka, 2016.

19. Ghaffarian, S., Gokasar, I., 2015. Traffic density measurement by automatic detection of vehicles using gradient vectors from aerial images. International Journal of Civil, Environmental, Structural, Construction and Architectural Engineering 2015, 9, 806-810.

20. Ghaffarian, S.; Ghaffarian, S., 2014. Automatic building detection based on supervised classification using high resolution Google Earth images. In the International Archives of Photogrammetry, Remote Sensing and Spatial Information Sciences, Copernicus GmbH, Vol. 40, p 101.

21. Ghaffarian, S., Ghaffarian, S., 2014. Automatic building-based map updating using classification-based change detection and snake algorithm. In ISPRS Technical Commission I Symposium, Sustaining Land Imaging: UAVs to Satellites, Denver, Colorado, USA, 2014.

22. Khalilizangelani, Y.; Ghaffarian, S., 2013. A Study of Geospatial Data Processing Based on Cloud Computing, 34th Asian conference on Remote Sensing, SC02, pp. 758-762, October 2013.

\section{Reports}

23. Lech, M., Ghaffarian, S., Kerle, N., Leppert, G., Nawrotzki, R., Moull, K., Harten, S., 2020. A Proof-of-Concept of Integrating Machine Learning, Remote Sensing, and Survey Data in Evaluations. The Measurement of Disaster Resilience in the Philippines. DEval Discussion Paper 1/2020, German Institute for Development Evaluation (DEval), Bonn. 